\title{
THE LANGUAGE OF THE
}

\section{MODHUPUR MANDI (GARO) \\ Vol. III: Glossary}

\author{
Robbins Burling \\ University of Michigan
}

Ann Arbor, Michigan April 2003 
(c) 2003 by Robbins Burling 


\section{INTRODUCTION TO VOLUME III}

This volume consists of an alphabetical glossary of the Mandi and A.chik words that appear in the first two volumes. The reader needs to understand the principles by which the words have been alphabetized:

1. Garo never uses $c$ except in $c h$. It would be possible to drop the $h$ entirely without introducing any ambiguity. In deference to Garo tradition I retain the $h$, but $c h$ can be thought of as a single letter and that is the way it is alphabetized.

2. Slightly more confusingly $n g$ is also alphabetized as if it were a single letter and as if it follows $n$ in the alphabet. Thus a word with $n$ will always precede an otherwise identical word that has $n g$. Sin - $a$ 'cold' precedes sing ${ }^{*} a$ 'ask question'. Less obviously, perhaps $\sin ^{\bullet}-k a-r i$ 'cold season' also precedes sing ${ }^{*} a$ because the $g$ in sing ${ }^{*} a$ is just one part of the letter $n g$, while the $k$ in $\sin \bullet^{-} k a-r i$ is a separate letter. This method of alphabetization has the advantage that it brings together words of related meanings.

3. Except for occasional use in borrowed words, where the original spelling is sometimes preserved, written Garo does not use the letters $f, q$, $v, x, y$, or $z$. Nor do most Mandis find it easy to pronounce the sounds that these letters represent in English or in other languages. They will not be found in the Garo words in this glossary.

4. Garo uses one extra letter that is not a usual part of the Roman alphabet. This is ".", and it is used to represent the raka (glottal stop). This is the symbol used for the raka in most Garo publications, although a raised circle or even an apostrophe is sometimes used instead. In the published Garo Dictionaries, the raka is consistently printed, but it is ignored for purposes of alphabetization. Whether $p a^{\bullet}$ - precedes or follows $p a$-depends on the letters that follow in the next syllable, and not at all on the raka. 
In this glossary, by contrast, $\cdot$ is regarded as a letter like all the other Garo letters, and it is alphabetized as the first letter of the alphabet, preceeding even $a$. This means that $s o^{\circ}-a$ precedes sok- $a$ 'arrive', because $\cdot$ occurs earlier than $k$ (or than any other letter) in the alphabetical sequence.

5. One other important principle must be kept it mind: Initial syllables are fully alphabetized without any influence from the second syllable, seconds are alphabetized without influence from thirds, and so on. For example, sa-ri 'sister-in-law' precedes sak-sa 'one person' because sa- precedes $s a k$-. Neither the $r$ of sa-ri nor the $s$ of $-s a$ has any effect on the alphabetization of the earlier syllables. Both so- $a$ 'rotten' and so-no- $a$ 'will be rotten' come before both $s 0^{\circ}-a$ 'burn' and $s 0^{\circ}-n o-a$ 'will burn' because so- comes before $s 0^{\circ}$-. Only when initial syllables are identical does the alphabetization of the second syllalbes become significant.

It may help to think of $c h, n g$, and even $l \cdot m^{\bullet}, n^{\bullet}$, and $n g^{\bullet}$ as if they are single letters, for that is, in effect, how they are alphabetized. The order of alphabetization, then, is: ', $a, b, c h, d, e, g, h, i, j, k, l, l \cdot m, m \cdot, n, n$, $n g, n g *, o, p, r, s, t, u, w$.

This method of alphabetization has the great advantage that it consistently groups words with identical syllables together, and this means that words with related meanings tend to be grouped together as well. The principles of alphabetization are illustrated by the following words that are arranged according to the alphabetization system used in the glossary:

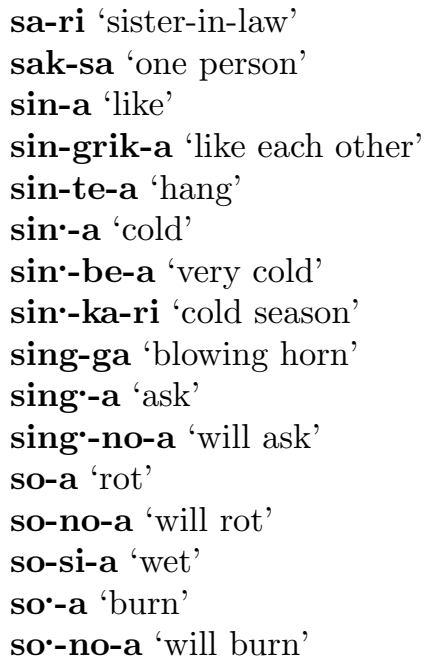


Glossary

so-ot-a 'kill'

sok 'breast'

sok-a 'arrive'

song-a 'set up a post'

song-a 'cook' 


\section{ABBREVIATIONS AND CONVENTIONS}

\begin{tabular}{|c|c|}
\hline$a a$. & adverbial affix \\
\hline$a d v$. & adverb \\
\hline cls. & classifier \\
\hline $\mathrm{cm}$. & case marker \\
\hline conj. & conjunction \\
\hline$c p$. & categorizing prefix \\
\hline dem. & demonstrative \\
\hline dns. & derivational noun suffix \\
\hline$e c$ & echo word \\
\hline int. & interjection \\
\hline$l w$. & locative word \\
\hline$n$. & noun \\
\hline$n s$. & noun suffix \\
\hline$n p h$. & noun phrase \\
\hline num. & number, numeral \\
\hline$p p$ & postposition \\
\hline ppp. & postpositional phrase \\
\hline pro. & pronoun \\
\hline pvs. & principal verb suffix \\
\hline$q w$ & question word \\
\hline ts. & terminal (verb) suffix \\
\hline$v i$ & intransitive verb \\
\hline vph. & verb phrase \\
\hline$v t$ & transitive verb \\
\hline lit. & literally \\
\hline esp. & especially \\
\hline plu. & plural \\
\hline$<\mathrm{B}$ & borrowed from Bengali \\
\hline$<\mathrm{E}$ & borrowed from English \\
\hline initial '-' & suffix \\
\hline final '-' & prefix \\
\hline
\end{tabular}


A-bima. The Modhupur Garos and their dialect

A.pal

The North Mymensingh Garos and their dialect

Arongga The area of the Garo Hills in India

A•chik Written Garo and the dialect upon which it is based

Mandi The dialect of Garo spoken in Bangladesh 


\section{GLOSSARY}

- int. no. (an ill-defined and quite variable vocalization including a rising intonation with slight voicing that is terminated abruptly with a glottal stop). (1.24)

- int. yes. (a vocalization that can be a vowel or an $\mathbf{m}$, with the distinguishing feature being a glottal stop in its middle). (1.24)

-a $\mathrm{cm}$. nominative case marker, marks the subject of a clause or sentence. Used only with monosyllabic pronouns: ang-a I. (0.12)

a int. isn't that so?, an expression that seeks and expects, agreement. This is characterized by a gently falling intonation, and it can have any fairly neutral vowel or even be represented by $\mathbf{m}$. (0.62)

-a pvs. neutral, unmarked tense-aspect marker. In addition to present time, it often shows habitual action, and can even imply past or future: kat-ing-a is running; ga-ak-a falls. (0.211)

-a pvs. nominalizing suffix that puts verbs into a form that is usable as a noun or as a modifier of a noun: dal-a me-a-sa big man. (0.218)

a- $c p$. a prefix used as a part of several kinship terms: a-bi elder sister; ada elder brother; a-chu grandfather; optionally with terms such as a-no younger sister. (6.64)

a-bat $n$. cultivation. $<\mathrm{B}(5.112)$

a-bat ko-ri-a $v p h$. cultivate. $<\mathrm{B}(5.112)$

a-bi, a-bi-gip-a, a-bi-tang $n$. elder sister, mother's elder sister's daughter, father's elder brother's son, elder cousin-sister. (6.615)

a-bi-a-da $n$. older sisters and brothers. (6.62)

a-bi-a-no $n$. women of one's own matrilineal group; sisters. (6.631)

a-bi-gip-i $n$. oldest sister, oldest of two or more sisters. (6.615)

a-bi-ong $n$. elder sister and her people. (6.63)

a-bi-sa $n$. a set of siblings, the oldest being female; a woman or girl and her younger brother or sister; wives of two brothers. (6.62) 
a-bi-sa ma-drang $n$. a set of sisters. (6.63)

a-bi-sa sak-sin-i $n$. the constellation of the Pleiades, (lit. seven sisters). (4.212)

-a-bo pvs. part of $d a \cdot$ - . . - a-bo the negative imperative. (0.215)

a-bu-a, ha-bu-a vi. bathe, take a bath. (6.57)

a-bu-dil-a, a-bu-it-a vt. give a bath, bathe (someone): ang-a pi-sa-ko a-bu-dil-jok I gave the child a bath. (6.57)

a-cha-ri-a vi. almost ripe, a bit riper than bri-a. (4.692)

a-chak $n$. dog. (5.35)

a-chak gol-dang $n$. a large type of dog. (5.35)

a-chak ka-si $n$. a castrated dog. (5.35)

a-chak pot-a $v t$. accuse someone of acting like a dog. (7.27)

a-da, a-da-gip-a, a-da-tang $n$. elder brother, mother's elder sister's son, elder father's elder brother's son, elder parallel cousin-brother. (6.614)

a-da-gip-i $n$. oldest brother, oldest of two or more brothers. (6.614)

a-da-ma-da $a d v$. so-so, (variant of al-a-mal-a). (3.453)

a-da-sa $n$. a set of siblings, the oldest of whom is male; a boy or man and his younger brothers and sisters. (6.62)

a-dal $n$. food given to animals, especially that given to pigs; bait. $<\mathrm{B}$ (4.487)

a-dal-a, ha-dal-a vt. feed animal, esp. pigs. $<\mathrm{B}(4.49)$

a-de, a-di, ma•-de, ma•-di $n$. mother's younger sister; father's younger brother's wife, step-mother. (6.612)

a-de-a-wang $n$. mother's younger sister and her husband; father's younger brother and his wife. (6.62)

a-di-ba-si $n$. aboriginal, tribal, native. $<\mathrm{B}(6.89)$

a-di-ta $n$. some, a certain amount, $(\mathrm{A} \cdot$ chik $)$. (3.346)

a-dik-sit-dik adv. urgent. (3.76)

a-dot $n$. original, genuine, real; personal name given by parents, in contrast to baptismal name. a-dot-e $a d v$. really, (same as a-sol-e). $<\mathrm{B}$ $(3.44)$

a-dot-ni bi-ming $n p h$. name given by parents; name actually used, as contrasted with baptismal name. (7.18)

a-dra $n$. half. (3.3700)

a-du-rak $n$. medium-sized tree with opposite leaves that are sour when cooked. (4.632)

a-du-ri, a-dru $n$. musical horn made from a buffalo horn attached to a long bamboo blowing tube. (8.85)

a-dul-i, a-dil-i $n$. eight annas, half a rupee. $<$ B (8.447)

a-ga-chi $n$. a beautiful flowering tree with huge leaves and slightly reddish pea-sized buds in January, and later yellow-red flowers, good red wood, trunk mottled with white, (Beng. $a-j u k-i) .<\mathrm{B}(4.632)$ 
a-ga-ja-ri $n$. a large tree with somewhat whorled leaves, (Beng. ja-rul). $(4.632)$

a-gal $n$. a kind of grass that makes long-lasting thatch. (4.652)

a-gal $n$. big fire, forest fire. (5.421)

a-gal-ni-gal $a d v$. from top to bottom, from here to there, all the way.

a-gan-a vt. talk, speak, say. (7.12)

a-gan-a-bak-bak-a vi. speaking a lot, continuously. (7.12)

a-gan-bing-bang-a vi. talk carelessly; talk without thinking, not seriously. (7.13)

a-gan-bu-a vi. speak falsely, say what isn't true, speak incorrectly; use broken language, as when Bengalis try to speak Mandi. (7.25)

a-gan-chak-a $v t$. answer, reply: nang-ni ko-ta-ko ang-a a-gan-chaka I answered your words. (7.32)

a-gan-chong-mot-a vi. speak correctly. (7.13)

a-gan-chrak-a vi. speak forcefully, speak openly, without holding back. (7.14)

a-gan-dik-a vt. speak of things that are not good, and which it is not nice to speak about; say things badly, in an unpleasant way. (7.12)

a-gan-e don-a vi. announce, inform, tell about an event beforehand; inform of one's intention. (7.34)

a-gan-e ron'-a vi. inform, give information, tell. (7.34)

a-gan-et-a vt. cause to talk, make talk; tell, inform. (7.12)

a-gan-ga-gal-a vi. speak carelessly, speak quickly; speak before the proper time. (7.14)

a-gan-grik-a vi. talk to each other, converse. (7.31)

a-gan-jol-jol-a vi. go around and speak to everyone. (7.12)

a-gan-pa-a vi. speak along with. (7.31)

a-gan-prak-a vt. explain, speak out, say fully, hold nothing back; declare, proclaim. (7.34)

a-gan-rik-a vi. speak to someone who is passing by. (7.12)

a-gan-rik-rik-a vi. speak continuously, repeatedly. (7.14)

a-gan-sit-tong-a vi. speak some, partially. (7.13)

a-gan-so-a, a-gan-su-a vi. announce, tell ahead of time, speak first. $(7.34)$

a-gan-srok-a vi. tell a little but not everything, conceal. (7.13)

a-ge $n$. before, ago, in the past. $<\mathrm{B}(2.62)$

a-gen-drak $n$. a medium-sized tree with fruit that is edible if cooked, (Beng. ping-gi-la). (4.632)

a-git-cha $n$. another, as an another helping of food, another person. $(5.453)$ 
a-gre $p p$. except for, other than, in addition to, (follows genitive -ni: na-song-ni a-gri other than you all, in addition to you. adv. too, excessively. (1.16)

a-grip $n$. a snare for catching birds. (5.845)

a-guk, ha-guk $n$. grasshopper, (see ha-guk for compounds). (4.469)

-a-ha pvs. past tense marker, (A•chik). Mandi has no simple equivalent but uses -a-ming, formed from the neutral -a and the terminal suffix -ming. (0.211)

A-ha-hu-a $n$. the traditional festival, one part of which is a stylized whooping shout that sounds like a-ha-hu-a. (8.562)

-a-ha-jok pvs. past tense marker for relatively remote time. (0.211)

-a-ha-ming $t s$. locates a past event before some other event: re-ang-aha-ming had gone, $(\mathrm{A} \cdot \mathrm{chik}) .(0.312)$

a-him-a, a-ham-a vi. clear throat. (9.44)

a-je-a, a-ji-a vi. sing a chanting song done at a sacrifice or other celebration, usually along with rice beer drinking. (8.83)

a-jong, ma•-jong $n$. mother's older sister; father's elder brother's wife. (6.612)

a-jong-pa-jong $n$. mother's older sister and her husband; father's older brother and his wife. (6.62)

a-kam $n$. burned rice from bottom of a cooking pot. (5.46)

a-kon $n$. a large coarse plant with dusty white appearance, $12 \mathrm{~cm}$. stalks and variable leaves up to $12 \mathrm{~cm}$, inedible. Two kinds, one with white, one with lavenderish flowers. Used as a medicine to apply to skin. (4.656)

a-ma $n$. mother, mom. (6.612)

a-ma $n$. monetary principal, as contrasted with interest. (8.442)

a-ma $n$. vertical pieces in a basket or in split bamboo matting; lengthwize pieces in a mat. (5.854)

a-ma gal-a $v p h$. lose, take a loss in buying and selling. (8.442)

a-ma-a-pa $n$. mother and father, parents. (6.62)

a-ma-ang-jong $n$. members of one's own matrilineal kin group, (lit. mother-younger brother). (6.62)

a-ma-cha-bak $n$. maternal side of the family. (6.63)

a-ma-sa-ri $n$. women of one's husband's matrilineal kinship group. (6.631)

a-mak, ha-mak $n$. monkey, macaque. (4.418)

a-mak ki-pret ka-a vph. a way of tying together two crossing roof pieces, so that the visible parts of the tie strips run parallel, contrasting with ka-stong-a where the visible parts cross. (9.65)

a-mak rang-gol, ha-mak rang-gol, a-mak nang-gor $n$. langur, long tailed monkey, hanuman ape. (4.418)

a-mak-greng $n$. a kind of cultivated tuber, (lit. monkey bone). (5.27) 
a-mik-ka pro. an unknown or hypothetical person; someone. (1.64)

a-min $n$. glue. (5.835)

-a-ming ts. suffix formed from the neutral tense-aspect marker -a with the past-conditional -ming; indicates past time: ching-a $\mathbf{i} \cdot$-ang-a-ming we went. (0.312)

a-mon $n$. one of the three paddy crops, (June-December). <B (5.25)

a-mong $n$. a mother and her family. (6.63)

a-mu-a vt. sacrifice, make a ceremonial offering to the mit-e (spirits) of rice, curry, etc., place before an altar. (8.562)

a-mul, a-mul-o $n$. period of time; era. $<$ B (2.51)

a-mut-a $v t$. accuse. (7.36)

a-na-ri $n$. someone else; extra person; non-relative, outsider. (1.64)

a-na-ros $n$. pineapple. $<\mathrm{B}(5.23)$

-a-ni pvs. nominalizing suffix that forms abstract nouns from verbs: cha-a-ni food, from cha-a eat; chan-chi-a-ni ideas, from chan-chia think. (0.218)

a-ni, ma-ni $n$. father's sister, mother's brother's wife, mother-in-law. (6.612)

a-ni-sa, ma-ni-sa $n$. mother-in-law and daughter-in-law. (6.62)

a-no $n$. egg yolk. (4.475)

a-no, no, a-no-gip-a, no-gip-a, no-tang $n$. younger sister, mother's younger sister's daughter, father's younger brother's daughter, younger cousin-sister. (6.615)

a-no $\mathbf{k u} \cdot$-wang $v t$. an insulting way of referring to a stupid woman, (lit. little sister, big mouth). (7.27)

a-pa $n$. father. (6.611)

a-pa-at-chu $n$. father and grandfather. (6.62)

a-pa-bo-ning $n$. men of one's husband's lineage. (6.631)

a-pa-cha-bak $n$. paternal side of family, kinsmen on paternal side. (6.63)

a-pi n. clam. (4.458)

a-pong $n$. father and his people. (6.63)

a-pu $n$. a large type of snail, $4-5 \mathrm{~cm}$. (4.458)

a-ra $n$. a kind of plant that is grown and tended by a ko-bi-raj or guni-al (herbal doctor) and that is said to be used to kill people: a-ra gi-si dried a-ra, a slow acting form of the plant; a-ra git-ang fresh a-ra, a quick acting form of the plant. a-ra on-a give poison, as by an herbalist. $(5.29)$

a-ra-ra-a vi. be lacking; be without; often used for foods that lack something, such as spices or meat; empty, of pots. (5.401)

a-rat-a vi. bored; tired of. (7.94)

-a-ri- aa. just, simply, merely: si-a-ri-jok (he) simply died. (3.344)

-a-ring- $a a$. contraction of -a-ri- just, and -ing- progressive: dak-aring-a just doing. (0.41) 
a-ring-ga $n$. a large water lizard. (4.441)

a-ro $a d v$. more, additional, again. <B (3.348)

a-ro-a-ro $a d v$. in addition; more and more. $<\mathrm{B}(3.348)$

a-ro-ba conj. moreover, used only in positive sentences. $<\mathrm{B}(1.4)$

a-sa $n$. hope, expectation. <B (7.725)

a-sam-kat-a $n$. a kind of rang (musical gong). (8.85)

A-sar, A-sal $n$. a Bengali month that overlaps June and July. $<$ B (2.823)

a-sek-ki $n$. star. (4.212)

a-sek-ki pro. echo in a-mik-ka a-sek-ki someone. (1.64)

a-si $n$. fortune, fate, luck; augury, as when judged from an inspection of bird's intestines: a-si-mal-ja, a-si-nam-ja bad luck, bad omens; a-si nam-a good luck, good omens. (8.52)

A-siin $n$. a Bengali month that overlaps September and October. $<\mathrm{B}$ (2.826)

a-sok $n$. bamboo fish trap. (5.843)

a-sol-e $a d v$. really, truly, actually. $<\mathrm{B}(3.44)$

a-sol-ni bi-ming $n p h$. name given by parents, (lit. real name). (7.18)

a-song-a vi. sit, sit down, (A·chik). (9.345)

a-su, aus $n$. one of the three rice crops (March-July. $<\mathrm{B}(5.25)$

a-ta $n$. wheat flour; glue. $<\mathrm{B}(5.405)$

a-ta-pol $n$. custard apple. $<\mathrm{B}(5.22)$

a-ta-sil $n$. a metal ladle, spoon. (5.436)

a-tom $n$. stomach, (the internal organ, not the surface of the belly). (6.27)

a-tun, a-ton $n$. horizontal and lengthwise roof pieces, usually made from a split half of bamboo, on top of which the thatch is tied. (5.632)

a-wa, a-wa di·-sa, a-wa gen-da $n$. small baby, (A·chik, A·pal), (same as gen-da ). (6.822)

a-wa, ha-wa dem. that far one, the one way over there. (1.5)

a-wa git-chak $n$. new born baby, infant, (lit. red baby). (6.822)

a-wang, wang-gip-a $n$. father's younger brother, mother's younger sister's husband, stepfather. (6.611)

a-wek-a vi. soft, sticking together but not very sticky. (3.668)

a-wil-i $n$. a person without a fixed home, one who wanders. (8.41)

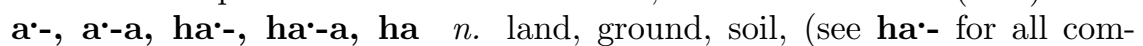
pounds). (4.242)

a-ba-cheng-a vt. begin. (9.13)

A-beng, Am-beng $n$. the Garo subgroup occupying the western part of the Garo Hills, as well as the Garos of Bangladesh. (6.89)

A-bi-ma $n$. the name that the Garos of Modhupur use for themselves. $(6.89)$

a'-bol, ha·-bol $n$. firewood. (5.423)

A--chik $n$. used by many Garos as a term for their own ethnic group, but used by the Modhupur Garos to refer more specifically to those living in 
the Garo Hills, and to the dialect that is perceived to be in use there. (6.89)

$\mathbf{a}^{\bullet}$-dam, ha-dam $n$. location, place, place to sit, site, house site. (2.11)

a-gop, gop-ang $n$. a large hole in a tree or in the ground. (2.38)

$\mathbf{a}^{\cdot}$-jak, ha'-jak $n$. reciprocal labor, labor to be paid back, labor exchange. $(8.433)$

a'-kam-pi $n$. shell of a snail (a-pi); snail shell used to scrape pots. (5.438)

a-kang-a, ra-kang-a $v t$. have a claim on a kinship group that should supply a spouse; claim an object. (6.651)

a'-kil-ok, ha'-kil-ok $n$. hole. (2.38)

a-kim, ha-kim $n$. relation between two lineages that is formed and perpetuated by marriage. (6.651)

$\mathbf{a}^{\cdot}$-kol, ha'-kol, ang-kol, hang•-kol a'-kil-ok $n$. hole, in ground or in any object, (cf. a--kil-ok). (2.38)

A-pal $n$. the word used by the Garos of Modhupur to name the Garos who live along the Indian border in the north Mymensingh area of Bangladesh. (6.89)

a'-pi-a vi. echo in chol-i-a a'-pi-a getting along, not poor. (8.441)

A'-rong-ga, $\mathbf{H a}^{\bullet}$-rong-ga $n$. the word used by the Garos of Modhupur as a name for the Garos of the Garo Hills. (6.89)

a'-sam $n$. edge, of cloth, book, etc., esp. the edge of a field, bank of a river. (5.87)

a•-sam jol-jol $a d v$. along the edge, as when walking, when planting crops. $(2.14)$

a'-sam-o $p p$. beside, next to, (follows genitive -ni): ang-ni $\mathbf{a}^{\mathbf{a}}$-sam-o beside me. (1.11)

$\mathbf{a}^{\bullet}$-sel, $\mathbf{a}^{\bullet}-\mathbf{s e l}-\mathbf{o}, \mathbf{h a}^{\bullet}-$ sel-o $p p$. because of, for the reason, (follows genitive -ni): in'-i a--sel because of this. (1.13)

a'-sik-a, ha-sik-a $v t$. want to, wish to, desire, (usually with an infinitive verb compliment). (7.725)

a'-ti, ha`-ti $n$. market, market place, bazaar. (8.449)

$\mathbf{a}^{\bullet}$-til-a, ha'-til-a $n$. courtyard, space between houses and buildings of a homestead. (5.644)

a-tot $n$. property of a single individual, including land, goods, rice, etc.; agricultural plot used by a single member of a family, often by a young unmarried person. (8.423)

a'-wal wat-a, ha'-wal wat-a $v t$. breaking of lineage bonds when divorce occurs, give up the rights to ha-kim, (lit. send away the land and fire (ha'-wal). (6.655)

$\mathbf{a}^{\bullet}$-we, a-we, we, u-e, ha'-we, ha'-wi, ho'-we, hu-e-ro, ho int. yes, (A•chik: we). (1.24)

ai $n$. mom, mother, (familiar and intimate). (6.612) 
-ai ts. strengthens a statement or suggests a correction to a previous statement: cha-jok-ai (I) have, indeed, eaten. (0.314)

ai-a int. ow!, an expression used when hurt. (0.62)

ai-au int. oh my, dear me, wow!, an expression of surprise, wonder, or dismay. (0.64)

ai-ma int. ow!, like ai-a, this is an expression of pain, but it is likely to be pronounced more slowly, and to be associated with pain that is less sharp but longer lasting. (0.62)

ai-na $n$. mirror. $<\mathrm{B}(5.82)$

ai-wa, hai-wa dem. that far one, that one way over there. (1.5)

ai-a, hai--a vt. know, understand. (7.41)

ak-, sak- cls. classifier for people, occasionally for ghosts, spirits, gods. (1.31)

ak-a vt. pick, pluck, flowers, fruit, leaves, etc. (5.16)

ak-gip-in pro. someone else. (1.64)

ak-git-tal $n$. newly picked, as new fruit. (5.16)

ak-ka-ru $n$. a kind of squash. (5.23)

ak-kai int. an expression indicating that one has misspoken, made a mistake; I mean. (0.64)

ak-ki, ak-ki-ak-ki, ak-ki-sa $n$. a little, a bit, a small amount. (3.345)

ak-ki-min'-chi $n$. a tiny bit. (3.345)

ak-ki-sa, ok-ki-sa $n$. a little: ak-ki-sa-ko ron-bo give a little. (3.345)

ak-mi-si-a vt. pick, pluck a lot. (5.16)

ak-mit-chi-a $v t$. tear off into small pieces, of paper, leaves. (9.294)

ak-pri-a vt. tear into pieces, of paper, leaves, cloth, especially to tear off the first piece. (9.294)

ak-ru-ru-a $v t$. pluck for a long time. (5.16)

ak-sa, ak-sa-han $a d v$. only, alone, of people. (3.344)

ak-sot-a vt. kill by twisting the neck. (6.79)

ak-tet-a vt. tear off, pluck off vigorously, of flowers, leaves. (5.16)

ak-tet-ga-gal-a, ak-ga-gal-a vt. pluck quickly, carelessly, incompletely. (5.16)

ak-tong-a vt. pull into two pieces, of cloth, string, ripe banana: break off bigger pieces by hand; kill a bird by stretching the neck. (9.294)

ak-tot-a vt. pluck off, of petals, etc. (5.16)

al-a-a vt. cultivate by plowing: ba-gan ge-na-skang al-a-a plow before planting the garden; bait-ko al-a-a, pal-an-ko-ba al-a-a plow the wet fields, also plow the dry fields. (5.154)

al-a-da $a d v$. different, separate. <B (3.435)

al-a-mal-a $a d v$. so-so. (3.453)

al-a-ni $n$. pole with cloth knot tied at one end used to push rice into the hole of a teng-ki (rice pounder). (5.438) 
al-a-si $n$. teasing about improper behavior, as when teasing a boy about his relationship with his grandmother. (7.26)

al-ai-a $v t$. sweep rice into hole of a teng-ki (rice pounder). $<$ B (5.451)

al-ak-si vi. selfish, stingy. (7.724)

al-ang $n$. flying green finger-sized leaf-eating insect. (4.469)

al-ap-a vi. talk, converse, discuss. $<$ B (7.31)

al-du-a $v t$. provide food for, feed; care for, support, of animals, children. (5.478)

al-ga $a d v$. uncovered, empty. $<\mathrm{B}(2.26)$

al-i, hal-i $n$. dike between rice fields. (5.114)

al-i- cls. classifier for a collection of four fruit, esp. for bananas. $<\mathrm{B}(1.35)$

al-ma-ri, al-mi-ra $n$. wooden cabinet for clothes, closet. $<$ B (5.82)

al-na $n$. rack for cloths and clothes. $<$ B (5.82)

al-nam-a vi. generous. (7.724)

al-ong-gi pal-ong-gi $a d v$. without possessions. (8.441)

al-pat $n$. wooden rice rake, a pusher with long handle and half moon shaped end for spreading rice to dry. (5.438)

al-si-a vi. lazy: al-si-a chik-a be bitten by laziness. $<$ B $(7.94)$

al-tu-a vi. easy, (A•chik). (3.75)

al-u-a vt. pound rice by the old method, without roasting it first. (5.451)

al-u-gu-ta $n$. white potatoes. $<\mathrm{B}(5.27)$

am n. mat. (5.852)

am- $c p$. prefix for kinds of mats: am-da-ri a fine kind of mat. am $n$. mat. (0.17)

am-a vi. roar, as a tiger. (4.476)

am-a, ham-a vi. manage, be able, win. (7.56)

am-bi $n$. grandmother. (6.612)

am-bi $n$. old yeast that is used as seed to make new yeast, (lit. grandmother). (5.405)

am-bi-at-chu $n$. grandfather and grandmother, old people, ancestors. $(6.62)$

am-bi-ri $n$. tree with a mottled trunk, sour but edible fruit and short almost needle-like leaves; as with a gim-bil tree, a cow may be tied to it by a dead person on the way to Chik-mang, the place where dead people go. (4.632)

am-bi-sa $n$. grandmother and grandchild. (6.62)

am-bil-et-tong $n$. tree with sour edible fruit. (4.632)

am-bin-o, am-bun-o, am-bin, am-bun $l w$. tomorrow, in the future, next, after a while. (2.65)

am-bin-o dong-e $a d v$. the day after tomorrow, the day after am-bin-o, (lit. omitting tomorrow). (2.65)

am-bin-o sal-sa-ni ja-man-o $l w$. one day after tomorrow, day after tomorrow. (2.65) 
am-bin-o-ni wal-ni-o $l w$. tomorrow morning. (2.65)

am-bin-o-ni wal-o, am-bin-o wal-o lw. tomorrow night. (2.65)

am-bing-gi, im-bing-gi $n$. reed for a sa-nai (musical horn). (8.85)

am-bun-o, am-bun, am-bin-o, am-bin lw. tomorrow. (2.65)

am-chak-a, ham-chak-a vt. stand, endure, put up with, often used in the negative: ham-chak-ja can't stand. (7.56)

am-da-ri $n$. a coarse kind of mat. (5.852)

am-pa-ti $n$. fine type of mat, the kind used to sleep on. (5.852)

am-pat-ti, am-pan-ti $n$. bird's gizzard. (4.471)

am-peng $n$. a wild but edible jungle tuber. (4.653)

am-pu-pu $a d v$. many, especially of piled up things, but also of cows, stars. (3.342)

am-ru-ri $n$. a good kind of mat. (5.852)

am-sok-a vi. confident, feel oneself to be capable; manage, have the ability, courage or patience for. (7.55)

am-sup-a-ri $n$. tree with flaky eucalyptus-like bark and sweet white edible fruit, (Beng. pe-ra, so-bri). (4.632)

am-tol, am-tol-a $n$. a rolled up mat, a mat ready to be put away; a mat placed in a large rolled circle as a place to store rice. (5.852)

am•-a, ham•-a vt. want, desire, ask for, (usually with a noun object); be about to, on the point of, almost, (see ham·-a for compounds). (7.725)

am•-bol cha'-a, ham-bol cha'-a vph. an itching skin disease, scabies. (8.953)

am•-pang $n$. thatch, thatching grass. (5.632)

am-pang-man-di $n$. a very tall type of grass that is the best type for thatch, thatching-grass. (4.652)

am-pang-pang-si $n$. a short variety of grass that is used for thatching. (4.652)

am•-pil-a, ham•-pil-a vi. turn, turn away, turn around; turn over, as in bed; reverse; vt. turn over, turn around, stir, sweep rice into a teng-ki hole. (9.327)

am·-pok, ham·-pok $n$. low stool. (5.82)

an-a $v t$. spread under, spread out, of things that go under something else, as mats, mattresses, banana leaves as plates. (9.62)

an-dal-a vi. be dark, dark of the night, of a closed room. (3.558)

an-dar-pen $n$. man's underpants. <E (6.527)

an-tal-a, jak-pa an-tal-a vi. hold the hand open, as to receive something. (9.46)

an-tek-ka $n$. an old-fashioned metal neck ornament worn as protection during headhunting. (6.563)

-an-ti dns. every: sal-an-ti every day; sop-ta-an-ti every week. (2.71)

an-ti $n$. week, market. cls. classifier for weeks, (A•chik). (2.53) 
an॰-, han॰-, han $n$. body, (usually used in compounds, for which see han'-). (6.21)

an'-cha-a vi. echo in a-je-a an•cha-a a chanting type of song. (8.83)

an'-cheng, han'-cheng $n$. sand. (4.242)

an'-chi, han'-chi $n$. blood, (lit. body water). (6.29)

an·ching pro. we inclusive, you and I, you and we, (A•chik). (1.62)

an-seng-a, han'-seng-a vi. happy, joyful; dance, celebrate. (7.721)

an-tam, an`tam-o $n$. late afternoon, evening, the time past midday.

an-tam-cha-pak $n$. the time before sunset. (2.52)

an-tam-git-tu $n$. dusk. (2.52)

an-tam-gro-gro, an·-tam-gru-gru $n$. the time around sunset. (2.52)

an-tam-pang $n$. evening star; dusk, evening, time after the sun has set but while it is still light. (4.212)

an-tam-pring $n$. all the time, (lit. evening-morning). (2.53)

an-tang, han-tang, an`-tang-tang pro. own, self, selves, oneself; the reflexive pronoun. (1.64)

-ang- $a a$. shows movement away from speaker, in that direction; bil-anga fly away. (9.312)

ang- $c p$. prefix used optionally with some kinship terms: ang-jong younger brother; ang-gri cross-nephew. (6.64)

ang-a pro. I; combining form: ang-: ang-ko me; ang-ni my. (1.62)

ang-bak $n$. close kinsman. (6.634)

ang-de, ang-di, de, di, de-gip-a, di-gip-a $n$. child as a kinship term, son, daughter, man's brother's child, woman's sister's child. (6.617)

ang-ga-ri $n$. echo in cha-wa-ri ang-ga-ri son-in-law. (6.614)

ang-gal $n$. dead coals, bits of charcoal, lumps of ash. (5.421)

ang-gri, gri, gri-gip-a, gri-tang $n$. man's sister's son, woman's brother's son, nephew. (6.617)

ang-gur $n$. grape. $<\mathrm{B}(5.23)$

ang-gut $n$. finger ring. $<\mathrm{B}(6.564)$

ang-jik, jik, jik-gip-a $n$. wife. (6.615)

ang-jong, jong-gip-a, jong-tang $n$. younger brother, mother's younger sister's son, father's younger brother's son, younger parallel cousinbrother. (6.614)

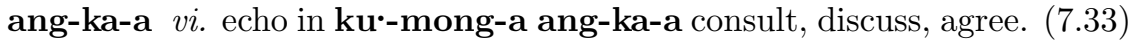
ang-nam-chik, nam-chik $n$. man's sister's daughter, woman's brother's daughter, niece, daughter-in-law. (6.617)

ang-sa-du, sa-du $n$. wife's sister's husband. (6.614)

ang-sa-ri, sa-ri $n$. sister-in-law. (6.615)

ang-se, se $n$. husband. (6.614)

ang-su, su·-gip-a $n$. grandchild. (6.617) 
ang-ti $n$. ring, finger ring. $<\mathrm{B}(6.564)$

ang·-ki, hang·-ki $n$. crab. (4.458)

ang•-ki-sa-a, hang•-ki-sa-a vi. open sore, skin ulcer. (8.953)

ang•-kol $n$. hole, (variant of $\mathbf{a} \cdot \mathbf{- k o l})$. (2.38)

ap-chon-a vi. narrow, of spaces, doorways, roads between houses, etc. (2.211)

ap-dal-a vi. wide, of spaces. (2.211)

ap-grang-a vi. widely spaced, made with open spaces between pieces, of baskets, etc. (2.38)

ap-san $n$. same, together, alike, (A·chik). (3.433)

ap-si-si $a d v$. very narrow. (2.211)

ar conj. and, in addition. $<\mathrm{B}(1.4)$

ar-ba conj. any more, used only in negative sentences as not any more: ar-ba dong-ja there isn't any more. (1.4)

ar-ki int. that is the way it is, concluding pause form, (lit. what else?). $<\mathrm{B}(0.66)$

at-cha int. fine, okay, right, yes. $<\mathrm{B}(0.66)$

at-chi-a vi. be born. (6.77)

at-chi-pil-a vi. be reborn, come to life again. (8.52)

at-chi-ram $n$. birthplace. (6.77)

at-ching $n$. stinging black ants that cause itching, bigger than sim-ilchak but smaller than ket-chi-ra. (4.465)

at-ching-a vi. sneeze. (9.45)

at-chong-a, a-song-a vi. sit, sit down. (9.345)

at-chong-brok-a vi. sit in a messy place. (9.345)

at-chong-chak-a vi. fit when sitting, enough room to sit. (9.345)

at-chong-chak-a-ni $n$. things for sitting, chairs, stools, etc. (9.345)

at-chong-chong-chrong-a vi. sit low, as on low stool. (9.345)

at-chong-chong-prot-a vi. squat. (9.345)

at-chong-chrot-a, at-chong-chong-chrot-a vi. squat, squat deeply but without one's butt touching the ground. (9.345)

at-chong-dam-bak-a vi. squat with butt touching something, as on a low stool. (9.345)

at-chong-dam-dam-a vi. sit for a long time. (9.345)

at-chong-dap-a vt. sit on something, cover something by sitting on it. $(9.345)$

at-chong-ti-ti-a vi. sit briefly, lightly; perch. (9.345)

at-chong-wek-a vi. sit badly, in a bad place, uncomfortably. (9.345)

at-chu, at-chu-gip-a $n$. grandfather. (6.611)

at-chu-sa $n$. grandfather and grandchild. (6.62)

at-tak-ka $a d v$. suddenly, quickly. <B (2.728)

at-te, at-ti $n$. large, machete-like knife that is used especially for cutting bamboo but also for general cutting and chopping. (5.832) 
at-te gam-ra $n$. a tool that is shaped very much like an ordinary at-te (bamboo knife) but that rests on the ground with sharp edge upward and that is used for slicing vegetables, etc., (same as bo-ti). (5.832)

au-a vi. bathe, $(\mathrm{A} \cdot \mathrm{chik}) .(6.57)$

aus, a-su $n$. one of the three paddy crops (March-July). $<$ B (5.25)

-ba ns. also. (0.14)

-ba- $a a$. shows movement toward speaker, in this direction: re-ba-a come; bil-ba-a fly in this direction, fly here. (9.312)

ba-, bat- $q w$. what? combining forms only, not used with the nominative but with most other case markers; bat- is used only before locative -cha and its augmented forms. (1.21)

ba-ang-a $n$. thin, of forest; empty of trees. (4.630)

ba-ba $n$. dad, father, (intimate). <B (6.611)

ba-ba-ri $n$. tree with red flowers and $25 \mathrm{~cm}$. round leaves said to cause a rash, (Beng. bew-la). (4.632)

ba-da ko-pi $n$. cabbage. $<\mathrm{B}(5.23)$

ba-dak-a $q w$. how?, what kind of? (1.21)

ba-dam $n$. peanut. $<\mathrm{B}(5.23)$

ba-di $q w$. how?, which way?: ba-di ra-ma? which road? (1.21)

ba-di-a vi. to exceed, be more than, go beyond, be bigger than. (3.348)

ba-di-sik qw. how much?, how many?: ba-di-sik-na? for what? for what price?; ba-di-sik-ni? how much? how much of it? (1.21)

ba-di-ta $q w$. how many, how much, $(\mathrm{A} \cdot$ chik $) .(1.21)$

ba-dik-e, ba-dik-i, bai-dik-e, ba-dik-e dak-a qw. how?, in what way?: ba-dik-e dak-e chu-no-a? how do (you) sleep? (on your back, side, etc.). (1.21)

ba-ding-a vt. peddle, travel about selling things. (8.444)

ba-du-ri-a vi. rain hard, pour, rain for a long time. (4.327)

ba-du-si du-a vph. swelling at the groin, at top of leg. (8.956)

ba-gan $n$. a garden, for perennial crops or for crops planted in careful rows such as tea, pineapple, sugar cane, flowers. <B (5.112)

ba-gi $n$. shares in sharecropping. $<\mathrm{B}(8.425)$

ba-gi ron-a, ba-gi on·-a $v p h$. give land to another to work in return for a $1 / 2$ share of the harvest; give to a sharecropper. (8.425)

ba-gip-a qw. which?, which of several?, of people or things: ba-gip-a me-chik? which women?; ba-gip-a-na ron-no-a? to whom will you give it?; ba-gip-a-ko ra-no-a? which will you take?; ba-gip-a-bagip-a? which ones? (1.21)

ba-git-a $q w$. in which way, by what route? (1.21)

ba-hi-ni $n$. a very large variety of bamboo, (same as rang-gi). <B (4.637)

ba-jar, ba-jal $n$. bazaar. <B (8.449) 
ba-je-a, ba-ji-a vi. tangled, disordered; messed up, dirty; caught, as in a trap or net; restricted, as not being allowed to enter a forest; caught between the teeth. $<\mathrm{B}(3.718)$

ba-je-tip-tip-a $v t$. tangle badly, as a string: ang-a do-ri-ko ba-ji-tiptip-a I tangled the string. (3.718)

ba-ji, ba-ji-o $n$. o'clock, hours of clock time. $<$ B (2.51)

ba-jin-a vi. to sound, make a sound, as a musical instrument: ang-a har-mon-i-ko ba-jin-et-a I play (cause to sound) the harmonium. $<\mathrm{B}$ (3.624)

ba-ju $n$. friend. (6.83)

ba-ju-bak-e $a d v$. going as friends, as side by side, holding hands, or with arms around shoulders. (6.83)

ba-ki bre-a $v p h$. buy on credit. (8.444)

ba-lu-ka-ja-wa $n$. type of bamboo that is good for basketry. (4.637)

ba-nai-a vt. prepare, build, construct, create, make; choose, select, appoint to an office. $<\mathrm{B}(9.13)$

ba-nai-e a-gan-ing-a vph. make up, not speak in a straight way, exaggerate. (7.13)

ba-no, ba-o qw. where? where at? (1.21)

ba-no-ba, bat-cha-ba, bat-cha-bai adv. somewhere, some place or other; with negative: nowhere, anywhere: bat-cha-ba dong-ja there are none anywhere. (2.13)

ba-no-na $q w$. where to? (1.21)

ba-no-ni $q w$. where from? (1.21)

ba-o, ba-no $q w$. where, where at?: bo-stu-ko ba-no don-a-ming? where (did you) put the things?; ba-no-ba-no where all (is it)?. (1.21)

ba-ra $n$. payment, rent, fare, bus fare. $<$ B (8.442)

ba-ra $n$. labor given in exchange: ba-ra ron-a give labor to be returned later; ba-ra ra-a receive labor from another that is to be paid back later; ba-ra chot-a repay labor. ba-ra-a vi. exchange labor, give labor in expectation of a later equivalent return. $<$ B (8.433)

ba-ra-grik-a vi. exchange labor back and forth. (8.433)

ba-ran-da, ba-ren-da $n$. veranda. $<\mathrm{B}(5.635)$

ba-rat $n$. long strips of hide that run along the length of a drum and tie the end pieces tight. (8.85)

ba-ri $n$. group of buildings built around a courtyard, compound; the group of people who live in these buildings. $<\mathrm{B}$ (5.622)

ba-ri-a vi. increase, as wages. $<\mathrm{B}(8.435)$

ba-ring $n$. eggplant, brinjal. (5.23)

ba-ring bel-a-ti $n$. tomato, (lit. foreign eggplant). (5.23)

ba-ring gi-sim $n$. kala azar. (8.92)

ba-sa, bha-sa $n$. language, way of speaking. $<\mathrm{B}(7.11)$ 
ba-sa-a, ba-si-a vi. float. $<\mathrm{B}(9.279)$

ba-sak-o $q w$. when? (variant of ba-sik-o). (1.21)

ba-sek-tom-iks $n$. vasectomy. $<\mathrm{E}(8.99)$

ba-si-a, ba-chi-a vt. sort out, choose, select; sort out what is not wanted; remove weeds, collect, of grass for cows; sort out unhusked grain; scoop oil from the top of a cooking pot. (9.536)

ba-si-ras $n$. variety of rice now in use, grown as an a-mon crop. $<\mathrm{B}$ $(5.25)$

ba-sik, bai-sik qw. how much? how many? (1.21)

ba-sik-o, ba-sak-o, bai-sik-o qw. when?: ba-sik-o-na? until when?; ba-sik-o-ni? since when?; ba-sik-o ba-sik-o when all? (A•chik: basok-o). (1.21)

ba-sik-o-ba, ba-sok-o-ba $a d v$. whenever, sometime; never (with a negative verb): ba-sik-o-ba re-ba-no-a will come sometime; ba-sik-o-ba re-ba-ja-no-a will never come. (2.71)

ba-sok-o qw. when? (A•chik). (1.21)

ba-sra, ba-sra ha-ba $n$. uncultivated but open area with short grass but without trees. $<$ B $(5.112)$

ba-sur $n$. calf, young cow. $<$ B (5.33)

ba-sur, ba-sul $n$. the smaller of the two grindstones, the stone that is held in the hand and used against the flat lower stone, (same as rong-te pi-sa). $<$ B (5.438)

ba-ti $n$. small bowl, saucer. $<\mathrm{B}(5.434)$

ba-wang-a, jak ba-wang-a vi. hold arms out wide, stretch arms straight out to the side. (9.38)

ba-a vt. give birth, bear child; carry child in cloth on hip, back or front. $(6.77)$

ba'-a, pa-a vi. perch, of birds; cling, of flies, as on a wound. (4.482)

$\mathbf{b a}-\mathbf{a}, \mathbf{p a}-\mathbf{a}$ vi. thin, of paper, etc. (2.215)

ba-deng-deng, ba-chi-deng-deng $a d v$. thin and flat, of cloth, paper, egg shells, etc. (2.215)

ba-dip-dip, ba-chi-dip-dip $a d v$. thin and flat, of cloth, paper, etc.; gone down, of a swelling. ba-chi-dip-a, ba-dip-dip dak-a vi. (2.215)

Ba-gip-a Bi-rom-bi $n$. the creator, the mit-e (spirit) who made the earth and who is now equated with the Christian God (I-sol). (8.54)

ba·-ra $n$. cloth, clothes. (6.594)

ba·-ra pin-a vph. covered with cloth. (6.598)

ba-rang-rang $a d v$. go down, of swelling. (8.956)

ba-rik-a $v t$. take care of, carry a baby or small child. (9.532)

ba-tom-a $v t$. carry a child in the arms or on the back without a carrying cloth. (9.532)

-bai ts. contraction of imperative -bo with emphatic -ai. (0.314) 
bai-a vi. last a long time, endure. (2.53)

bai-bai $a d v$. similarly, in the same way, as before, in a familiar way, same place, same condition, same time; serially, in order, one after another, along with. (3.433)

bai-bai-bai int. call to cattle to tell them to turn left when driving them. $<$ B $(4.49)$

bai-na bail, bai-na pat-i bail $n p h$. a trap for catching turtles. $<$ B $(5.843)$

bai-re $n$. outside. $<\mathrm{B}(5.644)$

bai-rep-rep $a d v$. one after the other. (2.14)

bai-sik, ba-sik qw. how much?, how many?: bai-sik-ni ro-a? how long?; bai-sik a-rim-jok? how late? Often used with a classifier plus -sa one: bai-sik kan-sa te-bil how many tables?; bai-sik gles-sa chi how many glasses of water?; bai-sik sal-sa? how many days? (1.21)

bai-sik ba-ji-jok, ba-sik ba-ji-a qw. what time is it? (lit. how much has (the clock) struck?). (1.21)

bai-sik-bai-sik, ba-sik-ba-sik adv. sometimes. (2.71)

bai-sik-o qw. when? (variant of ba-sik-o). (1.21)

bai-skup $n$. movies, cinema; an old fashioned word now laregly replaced by si-ne-ma. $<$ B $(8.83)$

bail, bai-na bail $n$. fish trap. $<$ B (5.843)

bait $n$. low land between higher banks, where rice can be grown, but that has water for only part of the year. $<\mathrm{B}(5.114)$

bak $n$. portion, part, share, as portions that are divided among several people. (3.347)

bak $p p$. side, position, in the direction of, (follows locative -cha): ama-cha bak on the maternal side; mik-kang-cha bak toward the front; $\mathbf{u} \cdot$-cha bak on that side. (1.11)

bak on-et-a, bak ku-ri-a vt. divide, as animals, as land to children in inheritance, give shares, (lit. give portions). (8.427)

bak-a, ha-bak-a vt. dig, chop out dirt in order to use it for something, often with a large ko-dal (digging hoe). (9.576)

bak-bak adv. quickly, (A•chik). (2.726)

bak-bak-a vt. cut off bark: bol-bi-gil-ko at-te-cha bak-bak-a cut off the tree bark with a bamboo knife. (9.573)

bak-chek-chek-a $v t$. cut a lot of dirt into small pieces. (9.576)

bak-gin-di-a $v t$. chop earth finely. (9.576)

bak-rok-a vt. scrape together with a ko-dal (hoe), as dirt, weeds, etc.; scrape, scoop out and move dirt. (9.576)

-bak-sa cm. with, along with, (A•chik). (0.12)

bak-so, ba-sko n. box. $<\mathrm{E}(5.82)$

bak-wak-a vt. scoop dirt, scoop out. (9.576) 
bal-a vi. bear flowers, bloom; pop, of corn. (4.691)

bal-a, bal-o-rot-a, bal-u-rot-a $v t$. carry in the mouth or beak, like a dog or bird: bal-e kat-a, bal-e ra-ang-a carry off in the mouth. (4.482)

bal-ang-ga $n$. empty of a cup, pot, a bus without people; not shut in, exposed to air, outside; open as a door or pot, an open truck: bal-ang-ga dak-a come open, as when a bottom of a glass falls off. (2.26)

bal-beng-a vi. float. (9.279)

bal-bik-bik, bal-chi-bik-bik $a d v$. very thin, of cloth. (2.215)

bal-chi-chi-a vt. echo in bal-ru-ru-a bal-chi-chi-a shake in the mouth, like a dog. (9.515)

bal-chi-ding-ding adv. as a gentle breeze, pleasant breeze. (4.36)

bal-deng-deng, bal-chi-deng-deng adv. thin, of cloth, fried bread. $(2.215)$

bal-iis $n$. pillow. $<\mathrm{B}(6.591)$

bal-iis-war $n$. pillow cover. $<\mathrm{B}(6.591)$

bal-im $n$. veranda of an earth house, earth platform, generally roofed, along the outside wall of a house. (5.635)

bal-ju-ri-a vi. float. (9.279)

bal-ma bu-su $n$. thorny shrub with opposite plain leaves. (4.64)

bal-mik-a vi. drizzle. (4.325)

bal-min-dri $n$. small circular wind that makes dust swirl. (4.36)

bal-o-rot-a, bal-u-rot-a vt. carry things in the mouth, as a dog or a bird. (4.482)

bal-ru-ru-a vt. mess up, jumble up, make a mess, as by searching; cause damage. (3.718)

bal-ru-ru-a $v t$. shake in the mouth, like a dog. (9.515)

bal-si-ri-a, bal-wa bal-si-ri-a vi. gentle breeze. (4.36)

bal-spu-a $v i$. open, of a bud, later stage than chong-a but before bal-a. (4.691)

bal-ti $n$. pail, bucket. $<\mathrm{B}(5.436)$

bal-wa $n$. breeze, wind. (4.36)

bal-wa mik-ka dak-a vph. wind and rain. (4.36)

bal--jak $a d v$. maturing of young people; having long hair; with widely spread branches. (6.78)

bam-a vi. bow, less deeply than bam-gok-a, lean forward, lean over from the waist. (9.348)

bam-a vt. sit on eggs, brood. (4.482)

bam-e dong-a $v p h$. bow down, crouch down, as when frightened. (9.348)

bam-e ra-ma re-a $v p h$. walk bent, as an old person. (9.348)

bam-gok-a, bam-gop-a, bam-gok-gok-a vi. bow head or body, bow deeply, bent over, of people. (9.348)

bam-kam-a vi. bent thoroughly over. (9.348) 
bam-kom-a vi. bow, curl up, curl up in fright. (9.348)

ban $n$. dam, barrier in a water channel. $<\mathrm{B}(5.114)$

ban-a vt. use a mon-tro (chant) to perform magic that can kill someone. $<\mathrm{B}(8.566)$

ban-chan $n$. sal tree, (Beng. gojari). (4.632)

ban-dang-a vi. too short, insufficient, of cloth, string, etc. (3.341)

ban-duk-ri $n$. tree with light bark and $10 \mathrm{~cm}$. leaves, (Beng. bas-ku-ra). $(4.632)$

bang $n$. carrying pole. $<\mathrm{B}(5.835)$

bang-bang adv. empty, of a hole or a building; clear of trees, open field; open door. (2.26)

bang-gu-ri-a vi. earthquake, to be an earthquake. (4.37)

bang-kang-a $v t$. prohibit, prevent, deny passage, obstruct. (9.77)

bang-si $n$. flute, played to the side. (8.85)

bang-a vi. much, many. (3.342)

bang-e $a d v$. a lot, much, very: bang•-e man-ja-no-a will not be able to do much. (3.342)

bang-e nam-ja $v p h$. not very good. (3.453)

bang-rik-rik-a vi. more and more, increase, of prices, things: chu bang-rik-rik-a-ring-a there keeps being more and more rice beer. (3.342)

bap-a-ri-bap, ba-ba, o-ba-ba int. exclamations showing surprise or wonder. $<\mathrm{B}(0.66)$

bap-tis-mon, bap-tis-mol $n$. baptism (for Catholics). $<\mathrm{E}(8.564)$

bap-tis-mon-ni bi-ming nph. Christian name, name given at baptism, as contrasted with name in daily use. (7.18)

bar-bar adv. again and again, repeatedly. $<$ B (2.71)

bas, bas gari $n$. bus. $<\mathrm{E}(5.86)$

bat-a vi. go beyond, cross water or a road, exceed, pass, be more, increase: ra-ma bat-jok crossed the road. -bat- aa. more than, exceed, go across: ga-bat-a step across, step over; jro-bat-a swim across; cha-bat-a eat more. (9.312)

bat-cha $q w$. where?, where to?, where from?: bat-cha dong-a where is (it)?; bat-cha-na re-ang-ing-a? where are (you) going?; bat-cha-ni i'-ba-ing-a? where are (you) coming from? (1.21)

bat-cha-ba, ba-no-ba $a d v$. somewhere; with negative, nowhere, anywhere: bat-cha-ba dong-ja there are none anywhere. (2.13)

Bat-dro, Bha-dro, Bat-ro $n$. n a Bengali month that overlaps August and September. $<$ B $(2.825)$

bat-e, bat-i $p p$. more than, (follows dative -na): ang-na bat-e dal-a bigger than me. (1.17)

bat-i $n$. oil lamp with small central wick. $<$ B (5.82) 
bat-il $n$. bow, crossbow, bow of a pong-si used in divination. < B (5.845)

bat-ol, bat-or $n$. dike between rice fields, (same as hal-i). $<\mathrm{B}(5.114)$

bat-tong-a, bat-tong-tang-a vi. take a shortcut. (9.319)

-be-, -bi- aa. very, a lot: jang-be-a very noisy; cha-be-a eat a lot. (3.342)

be-bak, be-bak-in n. all. <B (3.343)

be-be, bi-bi adv. truly, really, correctly. (3.44)

be-be ra-a, bi-bi ra·-a vt. believe, take to be true. (7.41)

be-bu, bi-bu $n$. a defective rice grain that has a husk but no inner seed. (4.677)

be-da-ku-ak na-tok $n$. kind of fish with large mouth. (4.454)

be-di $n$. church altar. $<\mathrm{B}(8.58)$

be-dol, be-dor $n$. mud, clay. <B (4.246)

be-ji $n$. mongoose. $<\mathrm{B}(4.419)$

be-ni $n$. braid. $<\mathrm{B}(6.561)$

be-pa-ri $n$. man who goes to villages to buy crops, pedlar. $<\mathrm{B}(8.41)$

be-ra $n$. the fence, as around a garden, esp. a fence made from bamboo matting, grass, or stems of sam-sim-a-ri plants: be-ra ka-a build a fence, (lit. tie fence). $<$ B (5.642)

be-ra $n$. sheep. $<\mathrm{B}(5.35)$

be-si, bi-si $a d v$. much, lots, more, very. $<\mathrm{B}(3.342)$

be-suk $n$. echo in o-suk be-suk sick. $<\mathrm{B}(8.91)$

be-wal, bi-wal $n$. habit, custom, convention. (8.14)

be-wal, bi-wal, ja-na be-wal, me-chik-ni be-wal $n$. menstruation, (lit. custom, monthly custom, women's custom). (6.72)

be•-a, bi-a vi. break, of solid things such as walls, pots; not used for linear things like string, etc. (9.282)

be-chil-ak-a, bi-chil-ak-a vi. cracked, as wood. (9.282)

be-chrak-a, bi-chrak-a vi. break off roughly, as branches from a tree in the wind. (9.282)

be-dem-a, bi-dem-a vi. folded, bent sharply, of harder things than dem-a, but not broken apart. (9.641)

be-dip-a, bi-dip-a vi. cracked, of glass, of a branch that is not separated. $(9.282)$

be-drak-a, bi-drak-a vi. break apart, of baskets, pottery, etc. (9.282)

be-el-a vt. take food out of one's mouth, to throw it away or to give it to a baby after premastication. (5.478)

be-el-e gal-a $v t$. spit up, of babies. (5.478)

be-el-e ron-a $v p h$. give food to a baby after premasticating it. (5.478)

be:-en $n$. meat, flesh, body; meat of a vegetable in contrast to the leaves. $(6.27)$

be-en-bi-mang $n$. whole body. (6.21) 
be-gop-a, bi-gop-a vi. bend over and break but not break apart. (9.212)

be-grop-a, bi-grop-a vi. collapse, of walls, houses, trees. (9.212)

be-king-ben-da, bi-king-ben-da vi. chipped, as the rim of a pot. $(9.282)$

be-ku-rak-a, bi·-ku-rak-a vi. completely broken up, as glass, a lump of earth, a chopped log, etc. (9.282)

be-ku-weng-a, bi-ku-weng-a vi. chipped. (9.282)

be'-prak-a, bi-prak-a vi. completely broken, as a pot, a tree, a dam in a water channel. (9.282)

be'-pri-a, bi-pri-a vi. break into small pieces, completely broken. (9.282)

be-rik-ken-a, bi·-rik-ken-a vi. chipped along the edge, dented, of iron tools, pots. (9.282)

be-si-ri-a, bi-si-ri-a vi. cracked, of a glass, cup, pot, etc. (9.282)

be-tong-a, bi-tong-a vi. break into two pieces, broken off. (9.282)

be'-tong-tang-a, bi-tong-tang-a vi. broken completely apart. (9.282)

be-wak-a, bi-wak-a vi. break down, collapse, of earth. (9.212)

bek $n$. bag, shoulder bag. $<\mathrm{E}(6.521)$

-bek-, -bek-bek aa. freely, forcefully: ron-bek-a give freely, even against the receiver's will; wa-bek-bek-a rain a lot, keep raining. (7.56)

bek-bek-a vi. rain heavily. (4.327)

bek-tok-a $v i$. short, of people, sticks, string. bek-tok, bek-tok-tok $a d v$. $(2.215)$

bel $n$. bell, part of a bicycle. $<\mathrm{E}(5.872)$

bel $n$. jackal, (Modhupur). (4.419)

bel si-jel $n$. large type of jackal with a long tail and black around its mouth, (Modhupur). (4.419)

bel-dom-dom $a d v$. very fat, strong. (6.48)

bel-ki $n$. piece made of thatching grass, split bamboo, or sam-sim-a-ri stalks, that fills the triangular gable area, above the mud end wall of a house. $<\mathrm{B}(5.636)$

bel-bok-bok $a d v$. fat, of people, cows, etc. (6.48)

bel-et-ti $n$. bel fruit from tree with $5 \mathrm{~cm}$. somewhat pointed leaves. $(5.22)$

ben-a $n$. small tree with fruit ripening in April is sold to traders, and from which oil is extractd, $25 \mathrm{~cm}$. hand like leaves. $<$ B (4.632)

ben-dok-dok $a d v$. short, of a person or of things. (6.46)

ben-dol-dol $a d v$. fat and fine, of small children. (3.455)

ben-ga-ri, ben-riik-sa $n$. flat-bottomed rickshaw, 'van' rickshaw for carrying goods. $<$ B $(5.86)$

ben-tong-tong $a d v$. short and fat, squat, of people, animals, round things. (6.46)

ben-tong-a vi. large, fat, of people, things. ben-tong-tong $a d v$. (6.48) 
beng $n$. frog. (4.447)

beng-bil-ek $n$. a small frog. (4.447)

beng-bil-ok $n$. a type of frog with a high-pitched croak that is a bit bigger than a beng-bil-ek. (4.447)

beng-bong $n$. a large type of frog, with a deep croak. (4.447)

beng-ki, bi-ja beng-ki $n$. beeswax. (4.475)

beng-a vt. block the way, prohibit. (9.77)

bep-sa, be-bo-sa $n$. business. <B (8.444)

bet-bet-a vi. call by a bull or male goat to a female. (4.476)

bet-ton $n$. pay, wages to a worker; school fee. $<\mathrm{B}(8.435)$

bi- $c p$. prefix for plant parts and internal body parts: bi-gil skin; bi-ka liver; bit-chi juice; bi-jak leaf. (0.17)

-bi-, -be- $a a$. very, a lot: jang-be-a very noisy; cha-be-a eat a lot. $(3.342)$

bi-a pro. he, she; combining forms: bi-; bi-ko him, her; bi-na to him, to her; bi-ni his, her. (1.62)

bi-a-a, bi-a ka·-a vt. marry, get married. <B (6.651)

bi-am-bong, bi-am-bing $n$. midsection of a bamboo or tree trunk, above the ja-ting base, but below the rik-i top. (4.670)

bi-ap $n$. place, location, $(\mathrm{A} \cdot \mathrm{chik}) .(2.11)$

bi-ba bon-chot-a vi. dead, (lit. breath is finished). (6.79)

bi-ba wat-a vi. breathe out, exhale; die. (9.45)

bi-bak $n$. stem, of fruit, leaf, flower. (4.670)

bi-bal $n$. flower, blossom. (4.676)

bi-bi, be-be adv. truly, really, correctly. (3.44)

bi-bik $n$. intestines; inner edge of bamboo that is opposite to the green outer edge. (6.27)

bi-bil $n$. afterbirth, (same as sa-tip). (6.29)

-bi-bom dns. rounded, of long things, cylindrical, not flat, of tree trunks, unsplit firewood, posts, people's arms, etc.: bol-bi-bom unsplit firewood. (2.319)

bi-chal, bi-char, bi-sar $n$. meeting to settle a legal dispute, village trial. $<$ B $(8.13)$

bi-chap-bi-chap $a d v$. lots, of piled up things, of children. (3.342)

bi-chu $n$. point, end, tip, of string, pen, knife, etc. (5.87)

bi-dang $n$. rice straw. (4.66)

bi-dil $n$. the long growing stem of climbing vines such as gourds, squash, etc., (same as bi-du). -bi-dil dns. vine. (4.670)

bi-dil bi-dil $a d v$. growing densely, of vegetables or fruit. (2.25)

bi-dil-ni sang-gong $n$. tendrils, (lit. bracelet of vines). (4.670)

bi-ding $n$. horizontal pieces of split bamboo, crosswise pieces in a mat. $(5.854)$ 
bi-du $n$. the long growing stem of climbing vines such as gourds, squash, etc., (same as bi-dil). -bi-du dns. vine. (4.670)

bi-du-ra $n$. horizontal encircling strips of a basket, horizontal cane pieces of a sling for a pot. (5.854)

bi-gil $n$. skin of humans and animals, bark, peel, pod, leather. (6.29)

bi-gop-a vi. fallen over and on the ground, of trees. (2.318)

bi-gra $n$. rice husks, corn tassels, dried and seedless pods of mustard, etc. that are useless and thrown away. (4.677)

bi-gro, bi-gru $n$. length, the longer dimension: bi-gro ro-a, gip-engpang kan·-dok-a length is long, across is short. (2.34)

bi-gro bai-bai bil-a vt. roll up, starting from long dimension. (9.645)

bi-gron $n$. pit of fruit, seeds in a pod of beans, of chili, etc.; not used for seed to be planted. (4.677)

bi-gron, ri-sip-il bi-gron $n$. testicle. (6.249)

bi-ja $n$. bee. (4.463)

bi-ja-a-ma $n$. a large variety of stinging bee that builds its hive along tree branches; a beehive of this type. (4.463)

bi-ja-bit-chi $n$. honey, (lit. bee-juice). (4.475)

bi-ja-kol $n$. beehive located in a hole in a tree; the kind of bee that makes these hives. (4.475)

bi-ja-ri $n$. a species of wild cat with long claws, a bit bigger than a domestic cat, edible. (4.416)

bi-ja-si-ik $n$. a stinging red bee-like insect that does not produce edible honey, (wasp?). (4.463)

bi-jak $n$. leaf. (4.675)

bi-jak ko-pi $n$. cabbage. (5.23)

bi-jol $n$. mucus; the pale material, without blood, that comes with a newborn baby. (6.29)

bi-jong $n$. grub of a bee, immature bee that lives in a cell of a comb; the part of the hive where the young grow. (4.474)

bi-ka $n$. liver. (6.27)

bi-ka so-a, bi-ka su-a vt. angry at. (7.766)

bi-ka so-bru-a vi. pretend to be angry. (7.766)

bi-kil-ap $n$. bark, shell husk of coconut, areca nut, etc.; harder than bi-gil. (4.673)

bi-kil-ap, do*-chi bi-kil-ap $n$. the membrane inside the shell of an egg. (4.475)

bi-king $n$. turtle shell; shell of an insect; do•-chi-bi-king eggshell; mes bi-king matchbox; sko-bi-king skull. (4.473)

bi-kit $n$. gallbladder; the jang-gi (soul, spirit) is sometimes said to reside in the bi-kit. (6.27)

bi-kop $n$. peel, of banana, etc.; segment of a garlic clove. (4.673) 
bi-ku $n$. tip, point of a knife. (5.87)

-bi-ma dns. female, of animals; do-bi-ma hen. (4.40)

bi-ma $n$. rice grains with husks still on, especially those few remaining after most have had their husks removed. (4.677)

bi-mang $n$. body of person or thing; shape, form; middle portion of an object, such as a glass. (6.21)

bi-mik $n$. inscribed decoration on bamboo consisting of parallel lines with crossed lines between them; the diamond pattern formed in basket weaving. (5.856)

bi-ming, bi-mung $n$. name, personal name, name given by parents or at baptism, not including the lineage name. (7.18)

-bi-pa dns. male, of animals: do-bi-pa cock, rooster. (4.40)

bi-pan-tu $n$. stem of a fruit or a leaf. (4.670)

bi-pang $n$. tree; a tree or bush that grows with a single trunk or central stalk; trunk of a tree or banana plant; stalk of even a small plant such as mustard. (4.630)

bi-pek $n$. small branch of a tree: a--bol bi-pek small firewood; bi-pekja-si twig, (lit. branch-toe). (4.671)

bi-pong $n$. handle of tool, such as a knife, etc. (5.87)

bi-pot $n$. danger, trouble, calamity, disaster. $<\mathrm{B}(7.764)$

bi-rang, bi-ja-bi-rang $n$. honeycomb. (4.475)

bi-ri $n$. stinger of an insect such as a bee. (4.472)

bi-ri $n$. small cigarette. $<\mathrm{B}(5.429)$

bi-rik-i $n$. top of a plant. (4.671)

bi-rom-bi-tom, rom-bi-tom-bi adv. lots, plentiful, of fruit on a tree, of things tied in bundles. (3.342)

bi-rong $n$. grain, the edible grain of rice that lies inside the husk; iris of the eye; coin. (4.677)

bi-rot $n$. pimple, boil, pox. (8.955)

bi-rot-a-ma $n$. large pimple, boil. (8.955)

bi-rot-ma'-mong $n$. boil, large variety of bi-rot. (8.955)

bi-rot-mi-song, bi-rot-mi-srong, bi-rot-mi-si-rong $n$. small and painful pimple. (8.955)

bi-sa $n$. friend, special friend. (6.83)

bi-sa ka-a vph. make friends, of men. (6.83)

bi-si-mang pro. they. (1.62)

bi-sil $n$. the green outer surface of bamboo, green edge of a bamboo strip. (4.679)

bi-sim-ol $n$. rice that ripens late and that is left at the first harvest to be collected later. (4.692)

bi-sit-eng $n$. swelling at a node of bamboo where a side branch grows out. (4.679) 
bi-sku, bi-suk-ku $n$. node of bamboo, swelling at a node of bamboo where a side branch grows out, thick base of the branch. (4.679)

bi-skut $n$. biscuit. $<\mathrm{E}(5.408)$

bi-sok $n$. shaft of a metal tool that is hafted into a handle. (5.87)

bi-song pro. they: bi-song-ko them; bi-song-ni their. (1.62)

bi-stil $n$. cream that rises to the top of milk, egg yolk. (5.409)

Bi-sut-bal, Bri-hos-pot-ti-bar $n$. Thursday. $<$ B (2.815)

bi-ta $n$. plot, house site, site of a group of buildings; a place without buildings. $<\mathrm{B}(5.644)$

bi-te, bi-ti $n$. fruit; pod such as that of mustard when seeds are still in place. (4.676)

bi-te, mik-ka bi-te $n$. falling rain, raindrops, (lit. fruit): mik-ka bi-te rong-ma-rong-ma ga-ak-ing-a big raindrops are falling. (4.327)

bi-tin $n$. piece, chunk, of meat, sugar, soap, sweets, etc. (5.453)

bi-tip $n$. nest, cocoon. (4.475)

bi-tom-bi-tom, tom-bi-tom-bi $a d v$. crowded, touching, of fruit on the branches. (2.25)

bi-top $n$. nests of some insects, such as wasps and biting red ants, that are made in folded tree leaves. (4.475)

bi-wa $n$. sharp edge of cutting tool. $<\mathrm{B}(5.87)$

bi-a vt. ask, request, pray. (7.38)

bi'-a, be·-a vi. break, of solid things, (see be•- for compounds). (9.282)

bi-am-a, bi-a $v t$. pray. (8.564)

bi-ap-a vi. pray. (8.564)

bi-chak-a vi. pray for someone, pray on behalf of. (8.564)

bi`-sa $n$. child, (A•chik), (same as di・-sa, pi`-sa). (6.822)

biis $n$. poison. $<\mathrm{B}(8.99)$

biis-na, bi-cha-na $n$. bedding. $<$ B (6.591)

-bik dns. intestines, internal organs: bi-bik intestines; bik-ma stomach; bik-chra- membrane of the intestine; do-bik intestines of a chicken. (6.27)

bik-a vt. carve, cut into shape, cut hair, carve a tooth on the top of a memorial post. (9.573)

bik-bat-a vi. make a mistake when talking, weaving, etc. (7.13)

bik-bi-grik $n$. halfway along a road, halfway point, middle point of a tree; half-filled, of a basket. (2.11)

bik-bik-a $v t$. shake, as yeast in a winnowing basket. (9.515)

bik-chat-a vi. be thick, of clouds. (4.323)

bik-chrap $n$. membrane of the intestines. (6.27)

bik-kot-a vt. extract, excavate, dig up, as root crops; show, reveal, as showing hands from the sleeve; take apart; discover, find out. (9.576)

bik-ma, buk-ma $n$. belly, stomach; body of a pot. (6.245) 
bik-ma gin-ang, buk-ma gin-ang nph. pregnant, (lit. stomach-with). (6.74)

bik-ma rat-a vi. pain and sickness associated with birth or menstruation.

bik-ma re•-a, bik-ma kat-a vi. diarrhea, (lit. stomach goes, stomach runs). (8.97)

bik-ma rong-dal-a $v p h$. round-bellied, having eaten well. (6.48)

bik-ma rong-ding-dang $n p h$. classificatory kinsmen, not real siblings, (lit. different bellies). (6.634)

bik-ma rong-sa $n p h$. from the same womb, true siblings, (lit. one stomach). (6.634)

bik-ong-kat-a vt. take out with the hand, as tubers from the ground, as the breast in order to nurse. (9.572)

bik-sring-a vi. cover thinly, of clouds. (4.323)

bil $n$. strength. (7.56)

bil- cls. classifier for hits or kicks. (1.38)

bil-a vi. fly, of birds, insects, airplanes; fly about in the air, of paper, leaves, etc. (4.486)

bil-a vt. dig out in order to get such things as tubers, bamboo roots. (9.576)

bil-ak-a vi. strong; grow serious, of a disease. (3.72)

bil-bang $n$. horizontal crossbeam of a house, just above the end walls at the gable, or spanning the side walls. (5.633)

bil-cha-a vi. fly up from a surface, take off. (4.486)

bil-chok-a vi. fly down. (4.486)

bil-ding-bil-dang $a d v$. restless, going this way and that; disorganized; not consistent; scattered, as when things go all over the place. (9.324)

bil-du-a vi. fly up. (4.486)

bil-du-a $v t$. dig up. (9.576)

bil-e int. I suppose, it is said. (0.64)

bil-et $n$. razor blade. $<\mathrm{E}(5.832)$

bil-jeng-jeng-a vi. fly with slowly flapping wings. (4.486)

bil-man-a vi. have strength to, have the means to; often used in negative as bil-man-ja lack the means. (7.56)

bil-neng-a vi. tired. (7.94)

bil-on-a vi. fly down. (4.486)

bil-ong-a, bil-ong-en vi. important, strong, very, serious, of a disease. bil-ong-e, bil-ong-e-in $a d v$.: bil-ong-e ni-ri-ing-a seriously quarrelling. (3.76)

bil-pak-pak-a vt. fly with flapping. (4.486)

bil-rak-a vi. strong, of a person. (7.56)

bil-ru-rat-a vi. fly back and forth. (4.486) 
bil-si git-al $n p h$. new year. (2.66)

bil-si git-cham $n p h$. last year. (2.66)

bil-si- cls. classifier for years. $n$, year. (1.33)

bil-wen-a, bil-wen-wen-a, bil-wil-wil-a vi. fly in circles. (4.486)

bil-a $v t$. roll up, as a mat; wrap. bi-gro bai-bai bil-a $v t$. roll up, starting from the long dimension. gip-eng-pang bil-a $v t$. roll up starting from the short dimension. gro-gro bil-a vt. roll up starting from the long dimension. (9.645)

bil-e-rep-a vt. bunch up, of cloth, paper. (9.645)

bil-e-rim-a roll. up neatly, of a mat, cloth, paper. (9.645)

bil-ik $n$. short green beans with narrow, edible pods. (5.23)

bil-ting-a vi. well wrapped, protected; well thatched, well fenced; having a well-tucked in cloth. bil-ting-ting $a d v$. (9.65)

bin-da-su-ba am--pang $n$. a tall grass that grows in clumps and has spreading seeds on top, and that can be used for thatch. (4.652)

bin-ek-a vi. crushed, of crops knocked down by cattle, etc.; squashed, of bananas, etc; ground up, broken up, as dry earth; softened, as cooked meat. (9.578)

bing dak-e chrok-a vph. jump high and long, (bing seems always to be pronounced with a distinctively high and long intonation). (9.318)

bing-bang, bing-bang-bing-bang adv. carelessly, disordered, tangled, dirtied; without control, abruptly, doing something without knowing, a bit crazy: bing-bang a-gan-ing-a talking in a careless, disordered way. bing-bang bol-a $v p h$. speak carelessly, thoughtlessly, in a confused way. -bing-bang- $a a$. carelessly; a-gan-bing-bang-a talk carelessly, without thinking; ja-bing-bang-a stumble; cha-bing-bang-a eat carelessly or in ignorance. bing-bang-a vi be tangled, disordered, dirty. (3.718)

bing-a vi. woven tightly, of cloth or baskets, without large spaces between pieces. (5.856)

bing-chip-bing-chip, bing-chip-chip adv. woven tightly, without spaces between the strips of a basket. (5.856)

bing-chip-chip-a, jak-si bing-chip-chip-a vi. with fingers close together. (9.46)

bing-ding-bing-dang $a d v$. rapidly, as on a bicycle. (2.726)

bing-si-bing*-si $a d v$. quickly, very fast, of running, of a strong wind. $(2.726)$

-bit- aa. bring along, take along: ke-bit-a take along something that is hanging; rim-bit-a take a person along. (0.49)

bit-a vt. make a hole in the earth or mud, by a worm or animal; burrow; pierce, penetrate. (9.571)

bit-chi $n$. juice, as fruit juice, sap, honey; egg. (4.674)

bit-chi, ri-sip-il bit-chi $n$. testicle. (6.249) 
bit-chi-ri, bit-chri $n$. seed for use in planting, not a general term for all seeds. (4.677)

bit-drak-a $v t$. spread slightly apart, as thatch, clothing: bit-drak-bitdrak-e nem-ing-a spread apart so as to search between. (9.649)

bit-e-de-o $n$. a ground-living bird (lapwing, plover). (4.43)

bit-in-a vi. back up, go backwards. (9.312)

bo $n$. bride, daughter-in-law. $<\mathrm{B}(6.617)$

-bo pvs. imperative: ring-bo drink!; a-song-bo-ne please sit down! $(0.215)$

bo-a, bu-a vi. swell up, of a body part. -bo dns. swelling: ja-bo swollen foot; git-ok-bo goiter; ri-bo swollen testicles. (8.956)

bo-bil $n$. enemy. (8.32)

bo-chor $n$. year, years. $<$ B $(2.53)$

-bo-da pvs. emphatic or abrupt imperative. (0.215)

bo-dol dak-a vph. exchange, trade. $<\mathrm{B}(8.449)$

bo-ga $n$. kind of tree, probably a locust, with locust like leaves and long, skinny, inedible pods. (4.632)

bo-gin-a $n$. the thin aluminum used for cooking pots. (5.88)

bo-ji, bu-ji $n$. man's older brother's wife, wife's older sister. $<$ B (6.615)

bo-kul $n$. variety of tree with fragrant flowers. $<$ B (4.632)

-bo-ne pvs. polite imperative. (0.215)

bo-ning, ang-bo-ning $n$. man or woman's younger sister's husband, spouse's older brother, reciprocal term between men. (6.614)

bo-rai-a vt. load, fill, put in; wrap up, as in sacks. $<$ B $(2.26)$

bo-rang, bu-rang $n$. small house or watching platform in the fields; tree house. (5.622)

bo-ri-bo-ri $a d v$. swollen, of the body. (8.956)

bo-ro, bu-ru, bu-ro $n$. one of the three paddy crops (January-June). $<\mathrm{B}(5.25)$

bo-ro-a $n$. a very large variety of bamboo that is very good for making baskets, (Beng. bu-rak). <B (4.637)

bo-ro-diin $n$. Christmas, (lit. big day). <B (8.564)

bo-ro-wan $n$. the upper level of class one in primary school, (lit. big-one). $<$ B,E $(8.6)$

bo-rop $n$. ice, snow. $<$ B (4.329)

bo-ti $n$. a tool that is very much like an at-te (bamboo knife) but that rests on the ground with sharp edge upward and may be held by foot, that is used for slicing vegetables, etc., (same as at-te gam-ra). $<\mathrm{B}(5.832)$

bo-a, bu-a vi. speak incorrectly, say something untrue, whether in error, in jest, or in order to deceive. (7.25)

bo'-a ra'-a vi. speak untruthfully, joke and have fun. (7.25)

bo-bru-a, bo-bru-e bol-a vi. fool around, joke. (7.25) 
bo-dap-a vi. pile a falsehood on a falsehood, exaggerate. (7.36)

bo-du-a vi. speak badly of, say bad things about, squeal on someone. $(7.25)$

bo-ol-a vi. rotten, of eggs. (3.688)

bo-rik-rik-a vi. speak falsely constantly. (7.25)

bo-srok-a vi. tell only part, not all, conceal, stingy with the truth. (7.25)

boi $n$. book. <B (7.28)

boi-om $n$. small, straight-sided pottery storage jar for salt, sugar, etc. $<\mathrm{B}(5.432)$

boi-os $n$. age, number of years one has lived. $<\mathrm{B}(2.51)$

Boi-sak $n$. a Bengali month that overlaps April and May. $<$ B (2.821)

boil-a $n$. old style large thick bracelets. $<\mathrm{B}(6.564)$

bok $n$. king stork, a long billed stork. $<\mathrm{B}(4.43)$

bok-a vi. white. echo in nam-a bok-a good; reflects the association of whiteness with goodness. (3.455)

bok-a $v t$. want, demand. (7.725)

bok-bak-a vi. boil vigorously, bubble up when cooking; suds up, of soap.

bok-bok-a-bak adv. big rolling boil. (5.46)

bok-chi-dim-dim-a vi. very white. bok-chi-dim-dim $a d v$. (3.531)

bok-chi-gam-gam $a d v$. clean and white, very white, of cloth, paper. (3.531)

bok-chi-jam-bok-chi-jam, bok-chi-jam-jam adv. thoroughly white, clean and white. (3.531)

bok-chi-pal-pal $a d v$. very white. (3.531)

bok-dim-dim-a vi. very white. (3.531)

bok-gip-bok $n$. largest kind of stork (bok). (4.43)

bok-jam-jam adv. clean and white, as cloth, paper. (3.531)

bok-kal-dek $n$. a small type of black and white stork. (4.43)

bok-ku-bok-ku $a d v$. incompletely white, white here and there, as graying hair, a powdered face. (3.538)

bok-ku-ku-a vi. pure white, completely white: bok-ku-ku adv.: kin-i bok-ku-ku dak-jok hair has turned completely white. (3.531)

bok-nel-a vi. fade, of colors; whitish, somewhat white, dirty white. (3.531)

bok-pal-pal $a d v$. pure white, very white, clean and white, of cloth, paper. (3.531)

bok-rim-it $a d v$. pale colored, pale green, pale rose. (3.531)

bok-sim-bok-sim adv. mixed white and black. (3.538)

bol $n$. wood; tree. bol- $c p$. categorizing prefix for names of types of trees.

bol- cls. classifier for trees, axes, bamboo knives (at-te), drums. (4.630)

bol-a vi. talk, inform. $<$ B (7.12) 
bol-a-chan-chok $n$. small forest tree with $12 \mathrm{~cm}$. pointed oval leaves that is used for rafters. (4.632)

bol-bi-jak $n$. leaf of a tree. (4.675)

bol-bi-pang $n$. tree. (4.630)

bol-bit $n$. common large tree with large plain leaves. Good for handles of bamboo knives, (Beng. poi-ru-ja). (4.632)

bol-bret $n$. a large forest tree with sumac-like leaves, and good heartwood. The leaves are cooked with pork to add flavor. (4.632)

bol-cha-a vi. tall, of people. (6.44)

bol-chek-si $n$. twig of a tree. (4.671)

bol-chon-a vi. small, of bamboo knife, axe, etc. (2.211)

bol-chu $n$. tree with a thorny trunk, long narrow leaves, and a cottony inedible fruit that ripens in February. (4.632)

bol-dail, bol-dal, dail, dal, dail-a, dal-a $n$. branch, limb, tree branch. $<\mathrm{B}(4.671)$

bol-dal-a vi. big, of drums, of tools with handles. (2.211)

bol-dim $n$. sprouts, green twigs, green stalks. (4.676)

bol-dim-it $n$. tree with dark trunk and no heartwood, (Beng. gan-di-goja-ri). (4.632)

bol-do-reng $n$. big tree with heart shaped leaves, (Beng. kai-ka). (4.632)

bol-do-tek $n$. plant that grows in trees and that has little red seeds that birds eat. (4.656)

bol-du-bak $n$. tree with roundish $10 \mathrm{~cm}$. leaves, good for firewood. (4.632)

bol-du-kak-ki $n$. large kind of tree that gives sap. (4.632)

bol-gan-tong, gan-tong, bol-tong $n$. log, stick. (4.672)

bol-gong $n$. broken tree, fallen tree. (9.212)

bol-gop-ang $n$. hole in a tree. (4.673)

bol-grik-a vi. converse, talk. (7.31)

bol-grim $n$. dense with trees. (4.630)

bol-gro, bol-gru, jang-gil bol-gro, jang-gil-ni bol-gro $n$. backbone of a human or animal; bol-gro ridgepole of a house. (6.28)

bol-gro-ni krong $n p h$. post holding up the ridgepole. (5.633)

bol-im-bol-tim $a d v$. vigorously, actively, of fighting by people, children, dogs. (8.36)

bol-kak-ka-ru $n$. big tree with mottled trunk and $3 \mathrm{~cm}$. leaflets on 20 cm. leaves. (4.632)

bol-kam-bi $n$. very top of a tree, top of the rik-i. (4.671)

bol-kil-ok $n$. hole in a tree. (4.673)

bol-kong-bang $n$. hole in a tree. (4.673)

bol-ma vi. big, of bamboo knives, axes, tools with handles. (2.211)

bol-mat-ra $n$. a large tree with long slender pods, inedible fruit, and 20 cm. oval leaves. Good for firewood, (Beng. dut-krus). (4.632) 
bol-na-gil $n$. an edible lichen that grows on dead and dried out trees and that can be eaten with men-da. (4.656)

bol-ot-chong $n$. stump of a tree, the part left when a tree is cut. (4.670)

bol-pang $n$. tree. (4.630)

bol-pek-chrap $n$. fork of a tree where limbs divide. (4.671)

bol-pil-eng $n$. tree with yellow flowers, little green five sided fruit, and $20 \mathrm{~cm}$. undistinguished leaves. (4.632)

bol-pru $n$. very large tree with horizontally marked trunk and tiny, almost fern-like leaflets on compound leaves, (Beng. ses-ra). (4.632)

bol-rik-i $n$. tree top. (4.671)

bol-sal $n$. sal tree, (Beng. go-ja-ri). (4.632)

bol-sep, bol-srep, bol-sep-pra, bol-srep-pra $n$. a meter tall shrub with willow-like leaves. (4.64)

bol-si, bor-si $n$. hook, fishhook. $<\mathrm{B}(5.843)$

bol-si-bret $n$. large tree with ugly crinkly $15 \mathrm{~cm}$. lozenge shaped leaves and inedible fruit. (4.632)

bol-sil $n$. very large tree with dusty trunk, leaves like a sal tree and good, very hard, hearwood, (lit. iron-tree). (4.632)

bol-sit-ong-a vi. speak briefly, partially. (7.13)

bol-srem $n$. large tree with small, somewhat holly-like, shiny green leaves, without fruit but cattle eat its leaves. (4.632)

bol-tong $n$. long stick, log; memorial post. (4.672)

bol-u-ri $n$. tree with $15 \mathrm{~cm}$. leaves, orange flowers that bloom in January, and inedible fruit. (4.632)

bom-bin-eng, bong-bin-eng $n$. bumblebee that stings and makes its nest in a tree hole. (4.463)

bom-bom, bom-bom dak-a $a d v$. lukewarm, neither very hot nor very cold, of water, food. (3.673)

bon-du $n$. friend. $<\mathrm{B}(6.83)$

bon-duk $n$. gun. $<\mathrm{B}(5.845)$

bon-a vi. done, come to an end, finished, used up. (9.14)

bon-chot-a vi. finished, of a job, etc; used up. (9.14)

bon-dik-a vi. run out, be finished, insufficient, not enough. (9.14)

bon-dil-a $v t$. complete, finish. (9.14)

bon'-grip-a vi. be finished, stop doing something. (9.14)

bon-jang-chi-a vt. stop in the middle, half way, half done; middle of life. $(9.14)$

bon-kam-a vi. completely done, completely finished. (9.14)

-bong dns. round, fat, large in the midsection: wa bi-am-bong midsection of bamboo; ok-bong-bong wider at the midsection than the ends; kal-bong someone who eats too much, a glutton; ja-ram-bong full moon; tim-bong wide, of rice fields. (0.16) 
bor-ga $n$. echo in ba-gi bor-ga sharecropping. <B (8.425)

bor-si, bol-si $n$. hook, fishhook. $<\mathrm{B}(5.843)$

bos-tu $n$. thing, object, $(\mathrm{A} \cdot$ chik). $<\mathrm{B}(5.81)$

bot-a vt. provoke, pick a fight, challenge, cause someone to be angry, annoy, quarrel. (7.36)

bot-a, jin-ang bot-a $v p h$. lend money or rice for interest. (8.446)

bot-a pal-a, bot-ing-a pal-ing-a vt. tempt, as a girl might tempt a boy by winking or by showing a bit of breast or thigh. (7.38)

bot-na, bot-ni $n$. spouted metal or earthen pot, used in latrine. $<\mathrm{B}$ (5.436)

bra-a vt. pour into, not necessarily to fill, as water into a pot. (9.276)

bra-bra $a d v$. in large numbers, of people, birds, dogs, etc. (3.342)

bra-dim-dim, bra-bra-dim-dim adv. lots, of chicks. (3.342)

brak $n$. ladle with a scoop, generally made from bamboo, used for stirring and serving, esp. for curry. (5.438)

brang-a vi. loose one's way, get lost. (9.538)

-brap- aa. strong, excessive, uncontrolled: mik-brap-a be dazzled with glare; ka·-brap-a taste badly, especially from too much salt; go-brapa throw, broadcast in an excessive or uncontrolled way; ching-brap-a shine, of a hurricane lamp, fire, etc. brap-a vi. taste too strongly, esp. of salt or soda. (3.72)

bre-a, bri-a vt. buy. (8.444)

bre-a, bri-a vt. spit out small amounts, as a bit of dirt, old betel cud. (5.478)

bre-gal-a vi. spit out and throw away. (5.478)

bre-git-chi-a, bri-git-chi-a vt. buy something bad, as when cheated. (8.444)

bre-o-a vi. an unpleasant taste like that of bel fruit or orange skins. (3.682)

bre-o-bre-o $a d v$. mildly bre-o-a, not strongly so. (3.682)

bre-o-mi-si-a vi. very bre-o-a, not good to eat. (3.684)

bre-pak-a, bri-pak-a vt. buy as one goes somewhere, buy along the way. (8.444)

bre-rik-a, bri-rik-a vt. buy from someone passing, as from a peddler. (8.444)

brek $n$. brake, part of a bicycle. $<\mathrm{E}(5.872)$

-brek- $a a$. soft, excessively soft: ki·-brek-a have diarrhea; rim-brek-a grab with the hands and crush, when eating; nom-chi-brek-a cooked too softly; ging-brek-brek having a runny nose. (3.664)

brem-brem, brim-brim $a d v$. mixed colors as in a striped, checked or plaid pattern, baskets made with both dark and light strips. (3.538) 
bren-tak-tak, bren-tik-tik, brem-tak-tak, brem-tik-tik adv. of mixed colors, with spots of varied colors or a delicate pattern, especially when seen from a distance. (3.538)

breng-a $v t$. cook in a bamboo tube, as is done at sacrifices. (5.46)

breng-gu $n$. small red or black ant living in the ground that bites and causes itching, similar to an at-ching. (4.465)

brens, bren-chi, bens $n$. bench. $<\mathrm{E}(5.82)$

bret-a vi. burst, burst open, split, crack. (9.282)

bret-bret $a d v$. pleasantly warm, of the sun, the weather. (3.673)

-bri num. four. bri-gip-a $n$. fourth. (3.3704)

bri-a vi. nearly ripe, riper than ga-dom stage, but not quite min-a. (4.692)

brik-a vt. scratch one's self to soothe. (9.554)

brik-et-a $v t$. scratch someone else to soothe. (9.554)

brim-brim, brem-brem $a d v$. of several colors, as in a striped, checked or plaid pattern, baskets made with both dark and light strips. (3.538)

brim-kong-tok, bring-kong-tok, brem-kong-tok adv. mixed colors, as red, white, black, etc., as a tiger, a three-colored dog. (3.538)

brin-a vt. mix, mix together, as rice and curry, cement and sand. (9.535)

bring $n$. forest, jungle, (A•chik: bu-rung). (4.630)

bring-chang-tok $a d v$. having mixed colors. (3.538)

bring-go-ra $n$. thicket, thick forest. (4.630)

-brok- aa. mess up, spoiled, rotten, dirty, unpleasant, tasteless, bland, faded: rim-brok-a mix with the hand; kit-brok-a stick the hand in and grab; su-brok-a stab with a spear; so-brok-a rotten, not good to eat; chi-brok-a tasteless, bland, insipid, watery, without enough sugar, salt, soda, etc., often used for rice beer; mik-brok-a fade, lose color, grow dull; at-chong-brok-a sit in a messy place. (3.686)

-brot- aa. rub, rub against: rim--brot-a kneed sore muscles, rub off so as to clean; ga-brot-a scrape with the foot; not-brot-a rub against something in order to scratch, as an animal does. (9.554)

-bru- $a a$. falsely, pretending; in-bru-a tell wrongly; jom-bru-a pretend to be sick; dak-bru-a pretend; ni-bru-a see, watch, without being noticed. (7.25)

-bu- aa. excrete inappropriately: git-tam-bu-a wet one's pants; ke•-embu-a defecate in clothes, on a mat like a baby, etc.; el--bu-a spit up, of a baby. (9.49)

bu-a vt. pierce, prick; penetrate by a bu-su (thorn); cut through the dirt, by a plow. (9.571)

bu-ba-ra-ra vi. unable to speak, dumb. (7.15)

bu-chrak-a, bu-su bu-chrak-a vt. scratch, as by thorns. (9.554)

bu-chu-ma $n$. old woman, (A•chik). (6.828) 
bu-de-pa, bu-di-pa $n$. old man, (A•chik). (6.828)

bu-del-a vi. stand on end, as the hair on a dog. (4.482)

bu-ja- cls. classifier for loads, bundles that can be carried such as loads of firewood, thatch, etc. $<\mathrm{B}(1.34)$

bu-jai-a vi. advise, encourage, urge. $<\mathrm{B}(7.37)$

bu-ket-a vt. pierce and stay in, as a thorn. (9.571)

bu-prot-a vt. pierce into. (9.571)

bu-pru-a $v t$. pierce through. (9.571)

bu-ra $n$. old man, husband. <B (6.828)

bu-ra-a vi. old, grow old, of men. (6.78)

bu-ri $n$. old woman, wife. <B (6.828)

bu-ri-a vi. old, grow old, of women. (6.78)

bu-rop-bu-top $a d v$. rough in texture, of wood, ground, etc. (3.662)

bu-rung $n$. forest, jungle, (A•chik). (4.630)

bu-su $n$. thorn, fish bone. (4.671)

bu-su-a vi. cough. (9.45)

bu-su-bal-ma $n$. a kind of tree with very large thorns, (Beng. mon-kanta). (4.632)

bu-su-du-bik $n$. somewhat rose-like vine with lots of little thorns, but different from bu-su-sil-kring. (4.655)

bu-su-sil-kring $n$. somewhat rose-like vine with lots of little thorns but different from bu-su-du-bik. (4.655)

bu-ti-a, bu-ti-ti vi. short and fat, squat, of people, animals, things. $<\mathrm{B}$ $(6.46)$

bu·-a, bo-a vi. speak incorrectly, say something untrue whether in error, in jest, or in order to deceive, (see bo-a for compounds). (7.25)

bu-dak-a vi. thick, long, plentiful, of hair. (6.561)

bu-dak-bu'-si $a d v$. with long uncombed unkempt hair. (6.58)

bu·-rang bu•-jang $a d v$. messed up, messy, of hair. (6.58)

bu•-rin-eng bu•-sin-eng, bu•-sin-eng bu•-sin-ang adv. messy, of hair, uncombed. (6.58)

bu•-rok-a vi. joke, tease. (7.26)

buk-ma, bik-ma $n$. belly, stomach. (6.245)

buk-re-han $n$. mortgage. $<\mathrm{B}(8.425)$

bul-ang-bul-ung $a d v$. open, with nothing. (2.26)

but-am, gut-am $n$. button. $<\mathrm{B}(6.598)$

But-bal $n$. Wednesday. <B (2.814)

but-bu-ri $n$. a large, wrist-sized vine with curved thorns that catch clothes. (4.655)

but-bu-ri bu-su $n$. small thorn tree with $2 \mathrm{~cm}$. round leaves and edible small round fruit. (4.632)

but-i $n$. cunning idea, plan; intelligence, cleverness. $<\mathrm{B}(7.44)$

but-ol $n$. bottle. $<\mathrm{E}(5.434)$ 
-cha $\mathrm{cm}$. instrumental case marker that marks the instrument or means by which something is accomplished, with, by means of: sai-kel-cha by bicycle, (A•chik: -chi). (0.12)

-cha $\mathrm{cm}$. locative case marker showing position in space, or movement toward or from a location; in, on, to, from: bat-cha re-ang-ing-a? where are (you) going? (A•chik: -chi). (0.12)

cha $n$. tea: cha-git-chak tea served without milk, (lit. red tea). $<$ B (5.409)

-cha- aa. having to do with emotions, probably derived from cha-a eat: mik-cha-a love, eat with eyes; kat cha-a ashamed; ka'-cha-a speak angrily, bawl out. (0.48)

cha-a vi. get up, rise, grow, develop; sprout, as from a tree stump. aa. get up, grow, come to life: ni-cha-a look up, with head up; di-cha-a get someone up from sleep; bol-cha-a tall; tang-cha-pil-a come back to life. (4.691)

cha-a $v t$. echo in dok-na cha-a or den-a cha-a attack. (8.36)

cha-bak-a vi. go to someone for pity, as a child who asks for comfort. (7.741)

cha-bi $n$. key. $<\mathrm{B}(5.82)$

cha-chrok-a vi. jump, jump up and down. (9.318)

cha-chu-ra $n$. whorl of hair, top of the head. (6.25)

cha-dat-a, cha-ting-a, cha-ting-dat-a vt. bump into, collide. (9.552)

cha-deng-a vi. stand, stand up. (9.346)

cha-deng-dip-a vi. stand for a bit, pause while walking. (9.346)

cha-gip-ak-a vt. climb with one's arms around, as when climbing a tree. (9.531)

cha-grik-a vi. get up to face one another in a quarrel. (8.32)

cha-kat-a vi. stand up, get to standing position; get up from sleeping, be standing. (9.346)

cha-kat-ru-ra-a vi. stand up and sit down repeatedly. (9.345)

cha-kat-sri-sra-a vi. stand up suddenly from a sitting or lying position. (9.346)

cha-kat-tim-a vi. stand up very quickly. (9.346)

cha-kol, chak-kol cha-kor $n$. worker, laborer. $<\mathrm{B}$ (8.41)

cha-kri, cha-ku-ri $n$. wage earning job, especially with the government. $<$ B (8.41)

cha-kring-krang-a vi. get up quickly, suddenly, throw off coverings while getting up quickly. (9.346)

cha-mi-sa-a, sa-mi-sa-a vt. wake someone up, arouse someone from sleep, get someone up. (7.97)

cha-mos $n$. spoon. $<\mathrm{B}(5.436)$

-cha-na $\mathrm{cm}$. toward, in the direction of, (an augmented locative). (0.12) 
-cha-ni $\mathrm{cm}$. from, in the direction from, (an augmented locative). (0.12)

-cha-ni-ko $\mathrm{cm}$. an augmented locative used with transitive verbs indicating the direction from: bat-cha-ni-ko ra-ba-a? from where was it brought? (0.12)

cha-pa-ta $n$. tea leaves. $<\mathrm{B}(5.23)$

cha-pil-a vi. grow again, grow back, as shoots from a stump. (4.691)

cha-ra, sa-ra $p p$. other than, except for, instead, without, (follows nominative): ang-a sa-ra i-ang-bo go without me. $<$ B (1.16)

cha-ram-a vi. worn out, no good anymore, of clothes, bag, football. (9.292)

cha-ram-pri-a vi. old of things that tear easily, of clothes, fishnets, thread. (9.292)

cha-ri $n$. large open bowl for holding bathing water. (5.432)

cha-song $n$. generation, era, one's time of life. (2.53)

cha-sri-sra-a, cha-kat-sri-sra-a vi. rise, jump up, get up suddenly from sitting or lying, as when startled. (9.346)

cha-ting-a, cha-ting-dat-a, cha-dat-a vt. bump into, collide. (9.552)

cha-tip-tip-a vi. gather in large numbers, flock, swarm, of animals or people. (4.482)

cha-trip-trip-e dok-a, cha-grip-e dok-a $v p h$. hit one person by many people, as people might do to a thief. (9.553)

cha-wa-ri, cha-wa-ri-gip-a, cha-wa-ri-tang $n$. son-in-law. (6.617)

cha-a $v t$. eat, nurse; eat away at, by water or by a disease; live by means of; erupt, come out, of a skin disease; receive monetary compensation as as a result of a legal case. (5.472)

cha-am $n$. mortar for pounding rice, the hole of a teng-ki (foot treadle rice pounder) that holds the grain. (5.87)

cha-am $n$. mortar for pounding rice. (5.438)

cha-bil-a vi. come to surface and open mouth, of fish. (4.482)

cha-bing-bang-a $v t$. eat bad things, from ignorance or carelessness. (5.472)

cha-brek ring-brek $a d v$. eat and drink when one is not supposed to, as when under a taboo or when food has been forbidden because of illness. (5.472)

cha-brok-a vi. spoiled by being bitten or chewed on. (5.472)

cha-chak-a $v t$. suitable for eating, have enough space for food, as a plate: rang-tal-o mi cha-chak-a the rice fits on a plate. (5.472)

cha'-che, cha'-chi $n$. rice husks, chaff. (4.677)

cha'-che gin-di, cha'-chi gin-di $n$. food for pigs made from rice husks. (4.487)

cha-chip-chip-a vt. mouth carefully in tasting, suck on something, such as a lozenge. (5.472) 
cha-chip-chip-e ron-a $v p h$. chew food ahead to give to a baby. (5.472) cha-chon $n$. last child, youngest of a set of siblings. (6.64)

cha'-chot-a vt. eat up, finish food: mi-ko cha-chot-a finish eating the rice. (5.472)

cha'-chot-chot-a, sok cha'-chot-chot-a vt. suckle, suck the breast. (5.479)

cha'-dam-a vt. eat regularly in the same place. (5.472)

cha-dik-a $v t$. not good to eat, taste bad. (5.472)

cha'-dil-a vt. feed. (5.472)

cha-e gal-a $v t$. not finish the food, eat part only, eat some and throw away the rest. (5.472)

cha-e ni-a vt. taste, test by tasting. (3.682)

cha-gal-gal-a, cha-ga-gal-a vt. eat quickly. (5.472)

cha-gek-a, jong cha-gek-a vi. have a serious skin disease, as a disease that covers the body. (8.953)

cha--jring-a $v t$. always eat, eat regularly. (5.472)

cha-kan-di-a $v t$. eat half, eat some and leave some. (5.472)

cha-kim-bak-a $v t$. eat just a bit and quit, eat in part, not finish. (5.472)

cha-kin-ap-a $v t$. good to eat. (5.472)

cha'-mong-song-a $v t$. eat enough. (5.472)

cha'-nap-a vi. echo in ni-to-a cha-nap-a good to look at and to eat, beautiful and tastes good. (0.5)

cha'-nap-a vi. taste so-so, not very good to eat. (3.684)

cha'-nem-a $v t$. eat sandy foods. (5.472)

cha-pa-rik-a dong-pa-rik-a $v p h$. eating and staying at someone else's place. (5.472)

cha'-pa-rok dak-pa-rok adv. survive by working here and there; inconsistent, lazy. (8.441)

cha'-pak-a vt. eat along the way, eat while going somewhere. (5.472)

cha-pek-a $v t$. eat constantly, everything. (5.472)

cha-pin-ek-a vt. crush and make soft by chewing. (5.472)

cha--ring-ja $v t$. eat late, eat at the wrong time. (5.472)

cha'-rok-rok-a $v t$. eat here and there, inconsistently, as ants eat wood. (5.472)

cha'-si-a vi. echo in cha'-dik-a cha'-si-a taste bad, bad to eat. (5.472)

cha'-sim-sim-a $v t$. eat up, finish. (5.472)

cha-skap-a vi. touching, in contact. (9.552)

cha'-sret-a $v t$. let slip from the mouth. (9.54)

cha-trip-trip-a vt. eat by many, as insects eat bamboo, cloth, books: cha-trip-trip-e chik-a bite by lots of people. (3.342)

-chak- $a a$. accept, receive: ra-chak-a accept, receive: $\mathbf{k u} \cdot$-ra-chak-a agree, promise; an`-chak-a take into the body, be sick; wang-chak-a 
open mouth to receive food from another; ni-chak-a midwife, one who sees and receives; nok-chak-a accept into one's home; rim•-chak-a catch a thrown object. (9.72)

chak-a vi. fit, have space for, not overflow; take shelter, from sun, rain. -chak- $a a$. fit, have enough space for: ga-chak-a fit, of the feet; jakchak-a fit in the hands, have time for; chu-chak-a fit onto for sleeping, as onto a mat, bedstead, of adequate size for sleeping: chu-chak-gip-a cho-ki a bedstead the size for sleeping; dong-chak-a fit, sufficient place for; cha-chak-a enough space for eating, for the food; at-chong-chak-a enough space for sitting, as on a bus. (2.22)

chak-a vt. support, stand by, help protect; speak well of someone: chak-a kang-a contribute money, rice or a pig as contribution to a wedding or sacrifice. chak-e-chak-e $a d v$. speak on behalf of, defend. -chak- $a a$. on behalf of, for another person, support: hai-chak-a be understanding, perceptive; ron-chak-a give help; a-gan-chak-a answer, reply; dokchak-a hit back; ka·-sa-chak-a sympathize; ku•-chak-a agree, promise, answer; bi-chak-a pray for someone; dak-chak-a help; ken-chak-a afraid for someone else; peng•-chak-a defend. (9.73)

chak-a vt. endure, put up with, suffer, bear, stand, take refuge: chakchik-a put up with, tolerate, endure, stand suffering. (7.56)

chak-a, kat-e chak-a vi. run away and hide, of a young man who doesn't want to marry, or of someone who deserts a spouse. (6.651)

chak-ka $n$. wheel. <B (5.872)

chak-ka-a vi. echo in ding-du-a chak-ka-a have a fever. (8.93)

chak-kol, cha-kol $n$. worker. <B (8.433)

chak-ku $n$. knife, dagger. $<\mathrm{B}(5.832)$

chal $n$. the sides or slopes of a roof, of which houses generally have two or four. $<\mathrm{B}(5.632)$

cham-bil $n$. a citrus fruit somewhat like a jam-bu-ra (pomelo). (5.22)

cham-bil $n$. rounded part of memorial post. (8.58)

cham-cho-ra, chan-cho-ra $n$. small sparrow-like bird, (grey tit, munia), (Beng. cho-roi). (4.43)

cham-e $n$. friends of the opposite sex, often with the implication of illicit behavior. (6.83)

cham-o $l w$. at that time. (2.52)

cham-pri-a vi. worn out, no good anymore, of cloth, clothes, string, bags, tires, footballs. (9.292)

cham-a vi. worn down; become dull, of tools; worn smooth, of shoes, stools, roads, pots, etc. (9.292)

chan-a vt. count. (7.31)

chan-a vt. track, follow tracks, as when hunting animals. (4.49)

chan-a $v t$. put on the fire, have on the fire, of a cooking pot. (5.46) 
chan-chi-a vt. think, believe, suppose. chan-chi-pil-a, gi-sik chanchi-pil-a vi. change one's mind. (7.41)

chan-cho-ra cham-cho-ra $n$. small sparrow-like bird, (grey tit, munia), (Beng. cho-roi). (4.43)

chan-dal $n$. gun, (same as bon-duk). (5.845)

chan-et-a $v t$. put a pot on the fire. (9.511)

chan-kol $n$. fireplace, (A•chik). (5.423)

chang- cls. classifier for number of times, the number of times something has been done. (1.31)

chang-a vi. know how, skilled, (A•chik). (7.55)

chang-a $v t$. physically block the way, as against animals; hinder. -changaa. close off, block off: ting-chang-a enclose, separate; dim·-chang-a make a partition. (9.77)

chang-bok-a vi. mixed colors but including some white, of cloth, some animals; a bamboo decoration where part of the green surface is scraped away from both sides of the nodes. (3.538)

chang-e-chang-e $a d v$. alternate times, every other time; sal-ha chang-echang-e every alternate day; man-di chang-e-chang-e every alternate person. (2.71)

chang-ket-a vi. squeezed into, crowded; tight, of bracelets, etc.; stuck, too tight to pass through; closed in and can't get out; stuffy, of nose, throat. (2.22)

chang-peng-a vt. block off, physically block the way. (9.77)

chang-rik-a vt. prevent from going, hold back; echo in peng•-rik-a chang-rik-a block off. (9.77)

chang-ro-a vi. tall, of people, trees. (2.213)

chang-tong $a d v$. half filled, of a basket, pot, etc.: gil-as chang-tong chi one half glass of water. (3.348)

chap, chap-ma-git-ok $n$. large fly that eats blood, especially of cattle. (4.462)

chap-a vt. place in layers, pile up, as leaves, cloth, paper; attach, join; put beside, put together; give a second wife to a man. -chap- $a a$. join, attach, pile up: ka-chap-a tie together; nang-chap-a touching; nangchap-grik-a touching each other; dong-chap-a give a second wife to a man; ra-chap-a take together; take something with something else; sik-chap-a sew together. (9.647)

chap-a, dong-na chap-a vt. give a second spouse; attach, join, put beside; pile up, as leaves, cloth, paper; attach, join. (6.652)

chap-chap adv. side by side, touching. (2.25)

chap-e rim-a $v p h$. bring as a second wife. (6.652)

chap-ma-git-ok $n$. a large fly that bites cattle, (same as chap). (4.462)

chat-a vi. dull, not sharp. (3.666) 
chat-a vi. thick, of cloth, books, thatch, clouds, etc. (2.213)

chat-chi $n$. kin, kinsman. (6.63)

chat-chi-ang-bak $n$. directly related kin, close kinsmen. (6.634)

chat-or, chat-ol $n$. woman's outer cloth, wrapped over the top part of the body and outside of the skirt. $<\mathrm{B}(6.526)$

chau-a vi. steal, commit thievery; do something in secret. (8.34)

chau-kok, cha-a-kok $n$. thief. (8.34)

che, che-che-che-che int. don't! an expression of dislike, disapproval and disgust; sometimes used with children as a reprimand. (0.62)

che-pang, chi-pang $n$. lower part of the belly, below the naval. (6.245)

che-ru-ru, chi-ru-ru $n$. bird that lives in holes in the ground, smaller than a dove, dark blue or black. (4.43)

che-sul $n$. grass growing in the fields that grows high in high water. (4.652)

chek-a vt. filter, stain, mix, sift, esp. for filtering rice beer. (5.455)

chek-chek $a d v$. surely, really, real, genuine; pure colored, of a single color. (3.44)

chek-rek $n$. bamboo post with split and flared top that can hold things, used in sacrifices. (8.58)

chel, chel-gok, chel-tong $n$. chest of the body. (6.245)

chel dal-a vi. have a large chest. (6.44)

chel-chik $n$. breastbone, especially of a bird or pig. $<$ B (6.28)

chel-i $n$. chips from chopping, small and thin. $<\mathrm{B}(4.673)$

chel-ting-a vi. have a large and deep chest. (6.44)

chel-a vi. far, distant. (2.11)

chem-a vt. cut branches into pieces, usually after the branches are off the tree. (9.573)

-cheng- $a a$. first, before doing something else: cha-cheng-a eat first, (before something else); $\mathbf{i} \cdot$-ang-cheng-a go first, go ahead; $\mathbf{a}^{\bullet}$-ba-chenga begin, (cf. -so-). (2.62)

cheng-a vt. make the base of a basket. (5.856)

cheng-chap $n$. cymbal. (8.85)

cheng-chon-a vi. narrow waisted, constricted in middle. cheng-chonchon $a d v .(6.46)$

cheng-dang-a vi. tall and thin, skinny, of a person; long and straight: do cheng-dang-a long-legged bird. cheng-dang-dang, cheng-dang-si-si adv. (6.44)

cheng-ga-ru, cheng-ga-ri $n$. flying insect, similar to, but larger than a po-da-neng or mai-jing-jing, that makes noise in April and May evenings. (4.469)

cheng-kin-a $n$. jawbone, mandible, pig jaws that are hung up as trophies. (6.28) 
cheng-kom-a vt. turn up the bamboo strips when starting the sides of a basket. (5.856)

cheng-o $l w$. before, earlier, some time ago, in the past, in earlier times. $(2.62)$

cheng-a vi. light in weight. (3.73)

cheng-a, han-cheng-a, han jak cheng-a vi. healthy, strong; slightly drunk, (lit. body-light). (8.91)

chep-a $n$. a small variety of edible turtle that lives in water, (Beng. kat-tu-a). (4.445)

chep-a vi. to go down, of a swelling, flatten. (8.956)

chep-a bi-king $n$. shell of a chep-a (small turtle). (4.473)

chep-chep-a vi. shallow, not deep, of water that has receded. (2.215)

chep-pi $n$. pocket, shoulder bag. (6.521)

cher-men $n$. union chairman, local elected leader. $<\mathrm{E}(8.12)$

ches-ta dak-a, ches-ta ka-a, ches-ta rim•-a, ses-ta dak-a vt. try, make attempt. $<\mathrm{B}(7.725)$

-chet num. eight, $(\mathrm{A} \cdot$ chik $) .(3.3708)$

-chet-, -chet-chet- $a a$. tightly: rim-chet-chet-a grab hard; gip-akchet-a embrace tightly; wen-chet-a wind around tightly. (3.72)

chet-chring-chet-chrang $a d v$. with holes, as very thin cloth such as gauze, of old thatch that one can see through. (2.38)

-chi $\mathrm{cm}$. locative marker showing position in space, or movement toward or from a location; in, on, to, from, (A•chik). (0.12)

-chi $\mathrm{cm}$. instrumental case marker that marks the instrument or means by which something is accomplished, with, by means of: sai-kel-cha by bicycle, (A•chik). (0.12)

chi $n$. water, liquid: mik-chi tears; han`-chi blood; bit-chi juice. chi- $c p$. categorizing prefix for watery things: chi-bol puddle; chi-da-ri flowing stream; chi-dek pond, wide place in a stream; chi-mik spring. (5.409)

chi-bi-ma $n$. river, (A•chik). (4.252)

chi-bil-ok $n$. a place where water collects, puddle, water hole. (4.253)

chi-bo-del-a vi. swell up, as legs after making a long journey. (8.956)

chi-bol $n$. puddle. (4.253)

chi-brek-a vi. watery, of rice beer; tasteless, bland, insipid. (3.686)

chi-brok-a vi. tasteless, bland, insipid, watery, without enough sugar, salt, soda, etc., often used for rice beer. (3.686)

chi-chang-chang, chi-chang-chi-chang adv. empty, of pots, etc.; having gaps in a field of planted crops; gone, disappear, of rice seedlings under water. (2.26)

chi-chi-a vt. shake, shake back and forth, as a tree to loosen fruit, as straw to let stalks and impurities blow away. (9.515)

chi-chi-top-a $n$. an inedible kind of fish that can puff up its belly. (4.454) 
chi-da-ri $n$. flowing stream. (4.252)

chi-dau-dau $n$. a black bird that is bigger than a dove. (4.43)

chi-dek $n$. pond, wide place in a stream. (4.252)

chi-dep-a, ging chi-dep-a vi. flat, of noses. chi-dep adv. (6.42)

chi-dik $n$. bird coop. (5.622)

chi-er, cher $n$. chair, (A•chik: cho-ki). <E (5.82)

chi-ga $n$. spring, place where water is obtained from a stream or pond. (4.252)

chi-gal-a vi. vomit. (8.97)

chi-git-ok $n$. narrow place in a water channel or river, (lit. water-neck). (4.252)

chi-gring $n$. a reddish finger-sized wasp that builds nests from dirt, sometimes on houses. (4.463)

chi-jim-a vi. damp, moist, as an earth floor in the rainy season, or from a slightly leaking water pot. (9.272)

chi-jong $n$. a large turtle, larger and flatter than a chep-a, (Beng. $k a-$ sim). (4.445)

chi-jong-man-di $n$. a kind of turtle, a kind of chi-jong. (4.445)

chi-jong-sim $n$. a type of black turtle, a kind of chi-jong. (4.445)

chi-ka-sim $n$. water that has been kept (often overnight) for use by a ko-bi-raj (herbal doctor). (5.405)

chi-kil-ang-a vi. bald, without hair. chi-kil-ang $a d v$. (6.26)

-chi-king num. ten, (A·chik). (3.3710)

chi-kol $n$. well of the type that has a hand pump. (5.642)

chi-mik $n$. spring, water source. (4.253)

chi-ni $n$. sugar. <B (5.405)

chi-ni-chip-pu $n$. a nonpoisonous water snake. (4.443)

chi-ra-ra $a d v$. watery, water everywhere. (9.272)

chi-rang-rang $a d v$. clear, of growing things; bare, of fallen leaves. (4.630)

chi-ret $n$. water channel leading to or from rice fields. (4.252)

chi-ret den-a $v t$. break $\mathbf{a} \cdot \mathbf{- k i m}$, break the connection between intermarried kin groups (chi-ret, water channel, is a metaphor for the connection). (6.655)

chi-rik-chi-rik $a d v$. a bit wet, of ground; a bit sweet, of food. (9.272)

chi-rik-ing, chi-rik-ong $n$. bank of a river, pond, etc., shore. (4.252)

chi-rin-ik $n$. rust, slippery coating that builds up on an unwashed pot or the surface of a wet courtyard. (5.88)

chi-ring $n$. small stream. (4.252)

chi-ring kal-dek $n$. small stream, esp. the upper end of a stream. (4.252)

chi-ring-chi-dek $n$. rivers and ponds, wet places. (4.252)

chi-rip-a a-pal $n p h$. an area that can be used for rice in the dry season but that has too much water for cultivation in the wet season. (5.114) 
chi-rong-a vi. clean, of hands, body, cloth, etc.; ground or fields cleared of grass, weeds, leaves. chi-rong-rong $a d v$. (3.714)

chi-rong-gil-a, chi-rong-gil-gil-a vi. clean, of cloth, house, etc.; cleaned and plastered, house, courtyard; clear, of grass, etc. chi-rong-gil-gil adv. (3.714)

-chi-rot- $a a$. a little, a bit: git-tam-chi-rot-a pee just a little; ke-emchi-rot-a defecate just a little. (3.345)

chi-rot-chi-rot $a d v$. a little, a small amount; little by little. (3.345)

chi-rot-ta $n$. bitter-tasting weedy plant, $60 \mathrm{~cm}$. tall with purplish leaves, that is planted, dried, and later boiled into a bitter drink that is good against malaria and kala-azar. (4.656)

chi-sam, chi a'-sam $n$. water edge, bank. (4.252)

chi-ska-pong $n$. water surface. (9.272)

chi-sol $n$. Christian cross. (8.58)

chi-sru-sru $a d v$. spill over, of water from a pot that is being carried; fall through a leak. (9.276)

chi-ti $n$. letter, written message. $<\mathrm{B}(7.28)$

chi-ting-kil-ok $n$. water hole, puddle. (4.253)

chi-ting-ting, chi-ting-ting-bil-ting-ting $a d v$. water-tight, of a roof, umbrella. (9.276)

chi-tok-tak, chi-tok-tak dak-a adv. dripping, of dew. (4.329)

chi-wa-sing $n$. a bamboo tube water container. (5.432)

chi-wak-a vi. cooked too soft, of tubers, etc. (3.686)

chi-a vi. sweet. $n$. sweets, candy. (3.682)

chi-a, bit-chi chi-a $v t$. lay eggs, by birds, insects. (4.482)

chi-cheng-me-seng adv. sweet and sour. (3.682)

chi-chik-chi-chik $a d v$. a little sweet. (3.682)

chi-chim-it-a vi. very sweet. (3.682)

chi-chu, si--chu $n$. bladder. (6.27)

chi-ka-wak-a vi. very sweet, too sweet. (3.682)

chi-rik-chi-rik adv. mildly sweet. (3.682)

chi-rim-bak-a vi. very sweet. (3.682)

chiit $n$. seat, as on a bus. $<\mathrm{E}(5.82)$

chik-a $v t$. bite, by people, mosquitoes, etc.; bite by mit-i (spirits) when they cause disease; biting cold; cause rash; attack, of laziness. (5.472)

chik-ak $n$. roof of the mouth. (6.22)

chik-ak-a vt. bite off. (5.472)

chik-ap-a, wa'-ring-o chik-ap-a vi. get caught in the teeth or on the roof of the mouth. chik-ap-chik-ap $a d v$. (3.718)

chik-chik-chrak-chrak adv. quickly, carelessly, messily, disorderly. (3.718)

chik-chip-chip $a d v$. very cold, personally. (3.675) 
chik-e gal-a $v p h$. bite off and spit out. (5.478)

chik-il $n$. reddish and very poisonous ground-dwelling worm without legs, that bites people, and can kill like a snake, as much as $20 \mathrm{~cm}$. long. (4.467)

chik-jim-a vi. adv. very cold, as water gets cold from standing, or like a tin-roofed house in winter. chik-jim-jim, chik-jrim-jrim $a d v$. (3.675)

chik-kap-a vi. grab or hold in the teeth; hold by squeezing. (9.44)

chik-kim-bak-a vt. take a bite from and leave some. (5.472)

chik-ku-rak-a vt. bite into pieces. (5.472)

chik-ok-a vi. very cold, of food or water. chik-ok-ok $a d v$. (3.675)

chik-pin-ek-a vt. chew hard, chew and crush. (5.472)

chik-pret-a vt. bite through, make a hole by biting. (5.472)

chik-sot-a vt. bite off, as a pig might bite off its rope. (5.472)

chik-tet-a $v t$. bite off a piece, eat as a dog eats by grabbing with the mouth. (5.472)

chik-tot-a vi. very cold, of hands, etc., of hail, of a tin-roofed house in winter: jak chik-tot-a-na wal-o hang-a for very cold hands, warm them at the fire. (3.675)

chik-wak-a vt. bite and hold on as an animal does, bite off pieces as one eats an apple or banana. (4.487)

chik-wak-a vt. bite off pieces, as one eats an apple or banana; bite and hold, as an animal does. (5.472)

chil-a vt. lift or carry on the shoulder or head, generally of heavy objects such as a log or a shoulder pole, lift with the shoulder. (9.532)

chil-do-a vt. lift up with the head or shoulders; come out from under a covering blanket. (9.532)

-chim $t s$. an $\mathrm{A} \cdot$ chik suffix that is equivalent, in part, to Mandi -ming, but not used for a simple past; past perfect, conditional, $(\mathrm{A} \cdot \mathrm{chik})$ : re-anga-chim had gone. (0.31)

chim-a-a $v t$. first of the three poundings of rice in a rice pounder. (5.451)

chim-chim cham-cham $a d v$. intermediate, neither very tall nor very short, neither fat nor skinny. (2.213)

chim-it-a vi. be asleep, of one's leg, etc., pins and needles. ja·-chim-it-a $v i$. asleep, of the legs. jak-chim-it-a vi. asleep, of the arms. (3.64)

chim-ong-a vt. gather things, collect; gather people together. (9.535)

chim-chim-a vi. damp, of wood, straw, etc. (9.272)

chin-a $v t$. offer, make a ceremonial offering to the mit-e (spirits) of rice, curry, etc., place before an alter; serve to people, as rice beer or rice. (8.562)

chin-a vt. wear, put on, get dressed, be dressed, esp. for clothes worn above the waist and for garments that are wrapped, shirt, chador, top cloth; ba·-ra-ko chin-a drape cloth to provide shade. (6.53)

chin-dik-a vi. not good for wearing, of an upper or wrapped garment. $(6.53)$ 
chin-to-a vi. good for wearing, of an upper or wrapped garment. (6.53)

ching-a pro. we exclusive, I and someone, but not you, us; combining form: ching-. (1.62)

ching-a vi. shine, not necessarily brightly, of sun, moon, fire, candles, flashlight, TV screen, gold; light up, flame up. (3.556)

ching-brap-a vi. shine brightly, of hurricane lamp, lightning, fire, shiny metal. (3.556)

ching-chet-ching-chet, ching-chret-ching-chret $a d v$. shine like a TV screen or reflections in metal, like oily hair; flicker, sparkle. (3.556)

ching-chot-ching-chot $a d v$. shine, shiney, like a TV screen, pig fat, beautiful cattle. (3.556)

ching-dim-dim-a vi. flame up strongly, burn vigorously. (5.425)

ching-it-a $v t$. light, as a lamp. (5.424)

chip-a vi. closed, of doors, boxes, eyes, etc. -chip- aa. without seeing, hidden, shut off: su-chip-a pierce into blindly, without seeing; git-tamchip-a went one's pants, wet one's bed; rim•-chip-a grab something not seen; sin'-chip-a cool off when the clouds cover the sun. (9.211)

chip-a vi. sufficient, enough, esp. for seasonings such as salt, soda, oil, etc. -chip- $a a$. sufficiently, completely, very: ding-chip-a very hot; so-chip-a burn completely. (3.341)

chip-bu rim-bu vi. messy. (3.718)

chip-ik-a vi. lie or sleep face down, a sleeping position of small babies. (9.343)

chip-ku-ek-a $n$. more than enough; too much, of seasonings such salt in a curry, of water when mixing mud for house building. (3.341)

chip-ok-a $v t$. second of the three rice poundings. (5.451)

chip-pu n. snake, general term. (4.443)

chip-pu-bol-gi-si $n$. a small snake that bites. (4.443)

chip-pu-gram $n$. a black poisonous snake. (4.443)

chip-pu-ja-da $n$. large snake, python. (4.443)

chip-pu-ri-ti $n$. cobra. (4.443)

chit-a vt. tear, of cloth or paper; split bamboo when making strips. (9.294)

chit-drak-a $v t$. tear off. (9.294)

chit-grang-grang-a $v t$. split clear apart. (9.284)

chit-ket-chi-a vt. split bamboo lengthwise, but at right angles to the inner and outer surface. (5.856)

chit-man-di-a vt. split bamboo parallel to inner and outer surfaces. (5.856)

chit-mit-chi-a $v t$. tear into small pieces. (9.294)

chit-pri-a, chit-pri-pri-a $v t$. tear into small pieces. (9.294)

chit-pri-pri-gal-a $v t$. tear to pieces and throw away. (9.294) 
chit-tong-a, chit-tong-tong-a vt. tear off, tear clear through. (9.294)

cho-a $v t$. row or paddle a boat. (5.86)

cho-a, cho-sam-a, cho-sram-a vt. winnow, use a winnowing tray. (5.451)

cho-ai-a vt. get oil from: wak-ni mit-im-ko tel cho-ai-ing-a get oil from pig fat. (5.458)

cho-bat-a vt. row across. (5.86)

cho-bi, so-bi $n$. picture. $<\mathrm{B}(5.82)$

cho-chong-a vi. sit, a child's word that is used mainly with and by children. (9.345)

cho-ka, cho-ka a-kol $n$. fireplace. (5.423)

cho-ki $n$. bedstead, wooden platform. $<$ B (5.82)

cho-prong-a vt. winnow. (5.451)

cho-sam-a vt. winnow. (5.451)

cho-sim-a, chos-ma $n$. eyeglasses. $<\mathrm{B}(6.521)$

cho-a vt. dig a hole, as for a post or to harvest root crops. (9.576)

cho--a, chu•-a vt. wrap in a banana leaf by folding it into a rectangular bundle, esp. for servings of rice. (5.453)

cho-bil-a, chu-bil-a, cho--bil-et-a, chu-bil-et-a vt. stir. (5.46)

cho-cho-a, chu-chu-a vt. feed a baby with food that one has already chewed. (5.478)

cho'-chok-a, chu-chok-a vt. tie up in a folded banana leaf bundle. $(5.453)$

cho-gep-a, chu-gep-a vt. tie up in a folded banana leaf bundle, esp. rice: $\mathbf{i}^{-}$-sal-o mi sa-e cho'-gep-a tie up the rice in a banana leaf. (5.453)

cho-om- cls. classifier for the amount that can be held in an open hand. (1.36)

cho'-ot-cho•-ot $a d v$. by sucking, on a straw, arm, etc.: cho•-ot-cho•-ot dak-e ring-a drink by sucking. (5.472)

cho-tep-a, chu-tep-a vt. wrap in folded banana leaves. (5.453)

Choit, Choit-tro, Choit-tri, Chot-tri, Chu-et, Chot-ro n. a Bengali month that overlaps March and April. <B (2.833)

-chok- aa. pointed: ran•-chok-a thin, scrawny, of people; ku·-chok-a pointed; ru·-chok-a long and pointed; ok-chok-a bulging, of a distorted circle; rep-chok-a, den-chok-a, rat-chok-a carve a point. -chok dns. long and pointed. ja-pa-ku-chok heel of foot; gol-chok arrow, stake; do - chok a kind of bird with a pointed back end. (2.312)

chok-a vt. write, $(\mathrm{A} \cdot \mathrm{chik}$ : si-a). (7.28)

chok-a vt. to spoon, lift with a spoon, put in with a spoon. (9.58)

chok-at $n$. door frame. $<\mathrm{B}(5.638)$

chok-chok-a vt. shake, as in a bottle; stir back and forth fairly slowly, as when cleaning things in a container with water, as a basket with fish. (9.515) 
chok-kel-a $n$. small earthen pot. (5.432)

chok-ken-da, chok-kan-da, chok-ken-ta $n$. cigarette butt. (5.429)

chok-mik-bru-a $v t$. write in part, not all. (7.28)

chok-on-a vi. jump down; set, of the sun. (9.318)

chok-on-o, sal chok-on-o $l w$. sunset, time when the sun goes down. $(2.52)$

chok-tim-a $v t$. write regularly, constantly. (7.28)

chok-wak-a vt. scoop up water with the hand, plate, spoon, etc. (9.277)

chol-a $n$. sewn shirt, as opposed to gen-ji (knit undershirt), sweater. (6.527)

chol-a vt. steal, rob. (8.34)

chol-kok $n$. thief. (8.34)

chon-a vi. small, little. -chon- $a a$. small, used with classifiers to form verbs meaning small, of things so classified: rong-chon-a small of round things; king-chon-a small of flat things. (2.215)

chon-kim-a vi. filled out but not yet tall, a stage of growth. (6.78)

chon-nik-a $v t$. look down on; look down on oneself, feel inferior, (lit. see as small). (7.43)

chon-a vt. finish, complete, use up. (9.14)

chon-drok-ben-drok $a d v$. short and fat, of people. (6.48)

chon-kam-a vi. completely finished. (9.14)

chong- cls. classifier for an entire banana plant that consists of several stalks. (1.34)

chong-brang-chong-mit-chang $n$. a snake with rings of colors that, it is said, can be counted in such a way that if one ends on chong-mit-chang it is good luck, but if ending on chong-brang it is bad luck. (4.443)

chong-brong $n$. clump with stalks that divide from the base, such as the several stalks of a banana plant often do. (4.671)

chong-dal-a vi. big, of tubers, of clumps of plants with many growing sprouts. (2.211)

chong-dang-dang $a d v$. long and thin, tall and thin. (2.312)

chong-go $n$. a ladder that is separate and movable, not attached; harrow. $<\mathrm{B}(5.835)$

chong-ma-chong-ma $a d v$. big, of things growing in clumps, tubers, bamboo, bananas, used when there are more than one. (2.211)

chong-rong-a vi. having the proper proportion of height and width, of a basket; not too high and not too squat. (2.319)

chong-a vi. sprout, grow up from roots or from a cut trunk. (4.691)

chong-a vt. pile up, make a stack, as of thatch, straw, firewood, ashes. (9.647)

chong-bak- cls. classifier for close bunches of growing fruit or plants, litchis, onion plants, beans. (1.35) 
chong-ket-ta $n$. butt of a cigarette. (5.429)

chong-mot $n$. actual, real. -chong-mot- $a a$. really, certainly. (3.44)

chong-pok-a vt. stack up, as plates, pots, glasses, etc. (9.647)

chong-prot-a vt. gather in piles. chong-prot-chong-prot $a d v$. (9.535)

chong-top-a vi. short, small, of clothes or houses: chong-top, chong-top-top $a d v$. (2.215)

chop-a vt. suck on snails to eat them. (5.472)

chop-chop-a vt. pound clothes lightly against a board or rock when washing them. (6.57)

-chot- aa. finish: cha'-chot-a eat up; bon'-chot-a finish a job, etc.; mat-chot-a finish. (9.14)

-chot-, -chot-chot- aa. slurp, smack: ku•-chot-a kiss, smack; ringchot-a, ring-chot-chot-a slurp, suck on a straw; cha-chot-chot-a suckle, suck the breast. (9.44)

chot-a vi. break, come apart, tear apart, disconnect, of linear things such as string. (9.282)

chot-a vt. pay off a debt of money, goods, or labor, return labor in labor exchange; give back. (8.446)

chot-chot-a vi. whimper, groan. (7.29)

chot-chru, chot-chu-ru, chot-ru n. small edible snail, 1-2 cm. (4.458)

chot-pri-pri-a vi. torn to bits, of string. (9.282)

chot-tip-tip-a vi. torn to bits, of string. (9.282)

chot-tong-a vi. broken off, broken in two, torn clear through, of linear things such as string. (9.282)

chra, chra-tang, chra-gip-a n. men of a man's wife's lineage. (6.631)

chra dal•-gip-a $n$. the important male member of one's wife's lineage. (6.631)

-chrak- $a a$. forcefully, uncontrolled: bi-chrak-a break off, as branches in the wind; $\mathbf{k i} \cdot$-chrak-a defecate vigorously; ken·-chrak-a comb vigorously, as to get out lice; pik-chrak-a pull out strongly, as a tree by its roots. (7.56)

chrak-chrak $a d v$. speak or act freely, without holding back, without fear. (7.14)

chram $n$. a tree with a very sour fruit, (Beng. cham-bol). (4.632)

chrap-chrap $a d v$. crowded together, as of people at a market; growing close together, of fruits and vegetables; wearing a lot of jewelry. (2.25)

chret dak-a, chret-chret dak-a $v p h$. shine suddenly, as the sun coming from clouds, a flashlight turned on; flare up, of a match, etc. (3.556)

chrik-a vi. scream, shout. (7.15)

chrik-e bol-a, chrik-pret-e bol-a $v p h$. speak very loudly, shout. (7.15)

chrik-pret-a vi. scream loudly; squeal, of pigs. (7.15) 
chrim-a vi. heavy, (A•chik: jrim-a). (3.73)

chring-chri-a vt. lift up, hang up, as cloth to dry. (9.253)

chrip-chrip $a d v$. completely wet or oily, drenched, full of oil. (9.272)

-chrit- $a a$. in stripes, striped; into long pieces: sal-chrit-a striped, as cloth, a tiger; den-chrit-a chop into pieces; mat-chrit-a cut slightly, scratch. (2.312)

chrit-a vt. split, as to cut into pieces with a bamboo knife; the split that comes after the first and coarser den-ku-rak-a, which is to split the bamboo into halves, but before the more delicate chit-a. (9.573)

chrok-a vi. jump, of frogs, children, etc. (9.318)

chrok-du-a vi. jump up. (9.318)

chrop-a vt. together, of parallel parts, such as fingers or legs; closed, of an umbrella. (9.349)

chru-chru $a d v$. in large numbers, of people. (3.342)

chu $n$. rice beer: chu-bit-chi undiluted rice beer, the first rice beer from the pot; chu ok-ka-a had enough rice beer. (5.403)

chu ok-ka-a vph. had enough rice beer (chu). (5.48)

chu-a vi. sleep. (7.96)

chu-a-a vi. high, tall, of trees, people, buildings, hills, mountains; rise, of a sloping road. $n$. height, one of the three spatial dimensions, along with gro length, and gip-eng width. (2.213)

chu-a-sim-ak-a vi. wake up. (7.97)

chu-ak-a vi. tall, of hills, buildings, people; high, of the position of fruit on tree, etc. (2.213)

chu-ak-si-si, chu-ak-ri-ri vi. very high. (2.34)

chu-chak-a vi. fit onto for sleeping, as onto a mat, bedstead, of adequate size for sleeping: chu-chak-gip-a cho-ki a bedstead the size for sleeping. (2.22)

chu-chan-a, nok chu-chan-a vt. mark out the location of house walls and posts in preparation for house building. (5.631)

chu-chik-a, chu-gop-a, chu-chik-gop-a vi. sleep curled up, with knees drawn up and face down. (9.343)

chu-dil-a vi. lead to sleep, put to sleep, as a child. (7.96)

chu-ga-ri-a $n$. the offering of rice beer when it is poured over other food offerings during a sacrifice. (8.562)

chu-kan-ta $a d v$. incomplete, of an object, a song; with the top missing, of a tree, bamboo). (3.347)

chu-kel-dap-a vi. sleep all day. (7.96)

chu-kin-ap-a vi. good for sleeping, comfortable; sleep well, comfortably. (7.96)

chu-nap-a vi. sleep together, of a man and woman, one acknowledged way of initiating a marriage. (7.96) 
chu-pel-gap-e chu-a $v p h$. sleep face down. (9.343)

chu-peng-a $v t$. echo in dong-peng-a chu-peng-a lie on a path to block it. $(9.77)$

chu-ram $n$. sleeping place, sleeping mat, (A•chik: tu-ram). (5.636)

chu-ri $n$. knife; long, thin, knife shaped and reddish internal organ of pigs, near the liver. $<\mathrm{B}(5.832)$

chu-rim-rim-a vi. sleep badly, tossing and turning. (9.343)

chu-sim-a vi. lie down and get wet or muddy, as an animal in a pond, a pig in the mud. (9.279)

chu-sim-ra $n$. the main post at the center of an old style Mandi house. (5.633)

chu-song-e ni-a $v t$. measure the depth of water. (9.277)

chu-stim-a vi. doze, nod off to sleep. (7.96)

chu-ti $n$. holiday, leave. $<\mathrm{B}(8.433)$

chu-wan-chi, chu-wan-ti, wan-chi, wan-ti $n$. yeast for making rice beer. (5.405)

chu-wek-a vi. over cooked, cooked with too much water. (5.46)

chu-chu-pil•-a, $\mathbf{c h u} \cdot \mathbf{- c h u - e ~} \mathbf{d u} \cdot \mathbf{- a} n$. chew and spit out food to prepare it for feeding to a baby. (5.478)

chu-eng-a vi. sharply pointed, as a needle, thorn, pointed stick. (2.312)

chu-gim-ik $n$. all, total, whole, complete; a good full life. (6.77)

chu-ong-a vi. sufficient, enough. (3.341)

chu-sok-a vt. accomplish, carry out. (9.14)

chuk-ti, chuk-ti-potro $n$. agreement, especially a written agreement, as with a worker about time and wages; promise. $<\mathrm{B}(8.435)$

chun $n$. lime, eaten with betel leaf and areca nut. $<$ B (5.405)

-da ts. particle that strengthens an imperative: nap-bo-da do come in! $(0.31)$

da-ba $n$. water pipe, for smoking. $<\mathrm{B}$ (5.429)

da-bil-a $n$. wrap around skirt, machine woven, and generally thinner and a bit shorter than a dok-ban-da. (6.526)

da-da $n$. older brother, term of address. (6.614)

da-gai $n$. playing field, a field marked by lines for playing games such as ha-du-du or football. $<$ B (8.81)

da-kait, da-kat $n$. dacoit, robber, bandit. $<\mathrm{B}(8.33)$

da-kon, da-kin-i $n$. a cover for a pot with a central knob to lift it by, lid. $<$ B (5.438)

da-ling-ja·-wek $n$. black water-living frog-like animal that puffs up in anger. (4.447)

da-ma $n$. drum. (8.85)

da-na-pa-ri na-tok $n$. a fish said to live in holes and that is similar to na-dang but smaller. <B (4.454) 
da-ni da-ni int. call to cattle to tell them to turn right when driving them. $<$ B (4.49)

da-ra $n$. man of bad character. (8.33)

da-ra-gang-get $n$. a bird, (rufous-backed strike). (4.43)

da-rang, da-rang-ba pro. many people, everyone. da-rang-ba with negative means nobody: da-rang-ba dong-ja there is nobody; da-rangko-ba nik-ja see nobody, (A·pal, A•chik). (1.64)

da-reng-a vi. very sharp. (3.666)

da-ri $n$. naughty girl, female adulterer. (8.33)

da-ri, am-da-ri $n$. a coarse kind of mat. (5.852)

da-wek-da-wek $a d v$. cooked mushily, with too much water. wek-a-weka vi. (5.409)

da-wil-a $v i$. round of objects, circular. da-wil-wil $a d v$. (2.313)

$\mathbf{d a}^{\cdot-}, \mathbf{d a} \cdot \mathbf{- o} a d v$. now. (2.61)

da'-a-ba, da'-o-ba $a d v$. again, now also. (2.71)

da'-al wal-ni, da'-sal wal-ni $a d v$. this morning. (2.62)

da'-al wal-o, da'-sal wal-o $l w$. tonight. (2.63)

da-an $a d v$. in a little while, a little while from now. (2.63)

da'-an-da-an $a d v$. often, again and again. (2.71)

da-an-o $l w$. a little while ago, just now. (2.62)

da-bil-si, da'-a bil-si $n$. this year. (2.66)

da'-nang int. oh dear!, alas!, how sad!, an expression of dismay or sympathy. (0.64)

da'-o-ba, da'-a-ba adv. now also, again, moreover, and in addition. $(2.71)$

da'-o-ro-ro $a d v$. now-a-days. (2.61)

da'-sal, da'-al, da'-al-o, da'-a-sal, da'-hal $n$. today. (2.65)

da'-sel-o $l w$. at an earlier time. (2.62)

da'-si wal-o $l w$. last night. (2.62)

da'-si-o, da'-si $l w$. a while ago, long ago, formerly, longer ago than da'-an-o. (2.62)

da'-ska-ri, da'-sik-ka-ri $n$. last year. (2.66)

da'-ska-ri chang-ha-o $n p h$. year before last. (2.66)

dai $n$. midwife. $<\mathrm{B}(6.77)$

dai-dai $a d v$. a lot: dai-dai mik-ka wa-ing-a raining a lot. (4.327)

dail, dal $n$. side branch of a tree or plant: dail mik-chil-a $n$. twig. (4.671)

dail, dal $n$. pulse, dahl. $<\mathrm{B}(5.23)$

dak-a vt. do, make, behave, act as; be like, look like. (9.11)

dak-am-a vi. work badly, leave unfinished. (8.433)

dak-bru-a, dak-am-bru-a vi. pretend. (7.25)

dak-chak-a $v t$. help. (9.73)

dak-dil-a $v t$. show how, lead. (9.73) 
Dak-gip-a $n$. name of a mit-e (spirit) that is coupled with Ta-ta-ra as Ta-ta-ra Dak-gip-a. (8.54)

dak-grik-a vi. fight. (8.36)

dak-jring-a $v t$. always do. (9.11)

dak-nam $n$. name actually used in daily address, usually the same as the a-dot-ni bi-ming that is given by the parents, as opposed to formal baptismal name. (7.18)

dak-ri $a d v$. almost, almost enough, almost done, not quite, not much left, not a great distance: dak-ri pin-jok almost thatched; dak-ri cha-jok eaten almost enough; dak-ri dal--jok almost old enough, almost grown. (3.341)

dal, dail, dal-a $n$. side branch of tree or plant. $<\mathrm{B}$ (4.671)

dal-an-nok $n$. brick or masonry house. $<\mathrm{B}(5.624)$

dal-dal adv. making noise, banging, of banging on tin roofs, bamboo pieces knocking together, water flowing on steep ground, of drums, of chopping wood. (3.624)

dal-ia n. dahlia. $<$ E (5.28)

dal-a vi. big, large. -dal--, -dal- $a a$. big, used with numeral classifiers to indicate big, of things so classified: king-dal-a big of thin flat things. $(2.213)$

dal-a-sot-sot $a d v$. conceited, boastful. (7.748)

dal-am-pa $n$. a man with a high opinion of himself, with a superiority complex. (7.44)

dal-ang-bol-chang $a d v$. having a large body, tall and stocky. (6.44)

dal'-dal, dal'-dal-ding*-ding $a d v$. fresh, of food, ready to eat. (4.690)

Dal-gip-a $n$. a big spirit (mit-e). (8.54)

dal--mi-si-a vi. very big. (2.213)

dal'-nik-a $v t$. look up to, respect; feel superior, (lit. see as big). (7.43)

dal'-pi-pi adv. very big. (2.213)

dal-pret-a vi. fat, wide, grow large rapidly. (2.213)

dal-ro-ro-a vi. grow rapidly, high, tall. (2.213)

dal'-sot-sot-a vi. grow rapidly; a challenge by children: 'I am bigger than you'. (2.726)

dam n. price. $<\mathrm{B}(8.442)$

dam- cls. classifier for places: villages, fields, houses, windows, doors. -dam dns. place: dong-dam living place; mang-ru-dam grave; damdam always at the same place. (1.31)

dam-beng $n$. crosswise and widely spaced pieces of floor support of a field house that rest on the ja-gra and support the wa-rong. (5.639)

dam-bi $n$. a portion of rice beer for one person, such as might be served in a bottle or glass and given to a guest. (5.403)

dam-bok-a vi. round, rather than pointed, of a face; blunt, dull, not sharp. (6.42) 
dam-bong $n$. an edible mushroom. (4.656)

dam-bu, dam-bu-bu, dam-bu-dam-bu, dam-pu, dam-pu-dam-pu $a d v$. a large quantity, lots, much; fat: sam-bi-jak dam-bu-bu lots of curry; dam-bu rak-rak good, fine, of fine fat children, animal or plants such as bamboo shoots. -dam-bu- $a a$. plentifully, lots: mil-dam-bu-a fat, of people, cattle; ring-dam-bu-a drink a lot; $\mathbf{k i} \cdot$-dam-bu-a defecate plentifully. (3.342)

dam-dam adv. always at the same place; dam-dam a-chong-a always sit in the same place; cha-na dam-dam am--ing-a always wanting to eat in the same place. -dam-dam- $a a$. regularly at the same place: cha-dam-dam-a eat at the same place; dong-dam-dam-a stay at the same place. (2.11)

dam-san, dam-han $a d v$. the same, together, in the same place. (2.25) dam-bak-a, dam•-bak-bak vi. stocky, squat, low, of children; low, flat, of noses like those of Mandis, of low bushy plants, the back end of a pen; not tall, of a gim-bi (water pot). (6.46)

dam•-bet-a, dam•-bet-bet, dam·-bret-a, dam•-bret-bret vi. short, of people; flat, of noses. (6.46)

dam-bol-a vi. big, chubby, of bamboo shoots, bananas, children. dambol-bol $a d v$. (2.213)

dam-bu rak-rak $a d v$. good, fine, large, of a fine fat child, of bamboo shoots. (6.48)

dan-dan-a vi. lean back, lean against. (9.349)

dan-dan-o $l w$. echo in se-pang-o dan-dan-o near: se-pang-o dandan-o al-ap-e gol-po-e dong-na nam-a to sit close, chatting and telling stories, is fine. (2.11)

-dang ns. plural marker: me-chik-dang women. (0.11)

-dang- $a a$. with sufficient time, often used with a negative: dang-ja with insufficient time; rim•-dang-a finish working, without interruption; cha-dang-a time to eat; a-lap-dang-ja can't manage to talk, because of interruptions, etc.; chok-dang-ja no time to finish writing. (2.53)

dang-a vt. cultivate, with a git-chi (small hoe), take out weeds. (5.156)

dang-brit-a $v t$. scrape backwards with feet, as a dog scrapes the ground or a bird scrapes when looking for food. (4.482)

dang-ga $n$. money, paper money, taka, (A•chik: tang-ka). dang-ga- $c l s$. classifier for takas, units of currency: dang-ga-bri four takas. (8.447)

dang-ga bi-rong $n$. coins, (lit. money grains). (8.447)

dang-kin-ok $n$. the four corner posts of an old fashioned central fire place. (5.633)

dang-ot-a $v t$. poke someone, touch with a finger to call attention. (9.46) dang-rok-a vt. scrape out dirt or weeds, but more gently than simple dang-a. $(9.576)$ 
dang•-a, tang•-a vi. fit, big enough, of clothes, bracelet; fit through, a door, a narrow place. (2.22)

dang-dang-a vi. spaced out, at some distance from one another, of fruit, houses, trees; separated, not touching, of people sleeping, sitting, walking. dang•-dang, dang•-dang-grik, dang-dang-dang-dang adv. separated, not touching, of people sleeping, sitting, walking. (2.25)

dang-kep $n$. tongs: sil-dang-kep iron tongs; wa·-dang-kep bamboo tongs; bol-dang-kep wooden tongs. (5.438)

dap-a vt. bury, fill a hole; cover; close eyes. -dap- aa. cover, cover over, pile on, wrap: ha-dap-a bury; grip-dap-a pile up, set on top; ru-dap-a pour over; on-dap-a put on top of crossing pieces when basket weaving; at-chong-dap-a sit on, cover something by sitting on it; pindap-a cover, as with a blindfold; smooth over furrows of a plowed field; sal-ko ga-dil-a pin-dap-ing-a the clouds cover the sun; mit-dap-a close, as a bottle; gan-dap-a tuck in; wil-wil-dap-a wrap around; onjet-dap-a put something heavy on top of something else. (9.576)

dap-ket-a vt. fill a hole with dirt, push mud into the cracks of a drying house wall; bury. (9.576)

dap-pu-a vt. bury, esp. to bury a body; fill a hole, put something small into the ground. (9.576)

dap-sul-a $n$. a type of at-te (knife) with a long curved blade. (5.832)

dat-a vt. pound into, as a nail, as dried fish into a bamboo container, as a stake into the ground; insert a tool blade into a handle. (9.571)

dat-ket-a $v t$. insert tightly and leave in, as nails, poles into a hole. (9.571) dat-sik-a vt. pound or push into, as post into the ground, dirt into a hole in the wall. (9.571)

de int. indication of agreement to a request. (1.24)

-de ns. shows mild contradiction to expectation, on the other hand, but. $(0.14)$

de, di, de-gip-a, de-tang, gang-de $n$. child, as a kinterm; son, daughter. (6.617)

de-bra, di-bra $n$. carrying cloth for a baby. (6.521)

de-bring $n$. illegitimate child, (lit. jungle child). (6.64)

de-but-a-ru bi-pang $n$. tree with whorled, vaguely poinsettia like leaves, planted by the forest department. $<\mathrm{B}(5.22)$

de-chap, di-chap $n$. twin. (6.64)

de-deng-a vi. standing, (children's language). (9.346)

de-jak-ra, di-jak-ra $n$. first child, oldest child. (6.64)

de-kim-bal, di-kim-bal $n$. one's next younger sibling. (6.64)

de-me-chik, de-me-chik-gip-a, di-mi-chik $n$. daughter. (6.617)

de-pan-te, di-pan-ti $n$. son. (6.617)

de-ros $n$. small green vegetable, lady finger, okra. $<\mathrm{B}(5.23)$ 
de-srong, di-srong $n$. twin. (6.64)

de-wal $n$. wall of an earth house. (5.638)

de-wal nok $n$. house built of mud. (5.631)

de·-a, di·-a vt. lift up, carry, raise; take photo; put away, move, especially bedding and clothes in the morning; lift the vertical strip while weaving a basket in order to slip the horizontal strip underneath; fly a flag; belong to named kin group, (e.g. Cham-bu-gong, Sang-ma), assign to a lineage (ma'-chong): ang-ko Nok-rek de'-a I am a Nokrek, I belong to the Nokrek lineage. (9.532)

de'-bri-a, di-bri-a vt. shake, toss, with hands, feet, or a tossing tool, to shake the rice grains off the stalk: ka-rail-cha ker-ko de-bri-a toss the rice straw with a tossing tool. (5.16)

de'-cha-a, di·-cha-a $v t$. get up, as a baby from sleep; lift up, help someone to sit up; put up, put away, of mats, etc. (7.97)

de'-chi-a $v t$. echo in de'-bri-a de'-chi-a shake, toss about. (9.515)

de'-de'-de'-de int. comforting sound made to a crying baby. (0.62)

de-ep-a vt. squeezed, or pinched and hurt, between two things, as by tongs, by a hammer, by a rice pounder. (9.531)

de-gang-a, di-gang-a vi. resting with one end on the ground. (2.317)

de-gang-git-a, di-gang-git-a $a d v$. slanting, as a partly fallen tree; sticking up, of a dog's tail. (2.317)

de•-gang-grik-a vi. leaning against each other, as two trees. (2.317)

de-gat-a, di-gat-a vt. lift something heavy, as a roof frame, put in place, load, help someone to pick up a load. (9.532)

de'-jit-a, de·-jit-a $v t$. move, put in a different place. (9.511)

de'-kang-a, di-kang-a, de'-krang-a vi. keep off the ground, hold in the air, as a foot, or as someone sleeping with one foot off the bed. (2.317)

de-on-a, di·-on-a $v t$. remove from the fire, as a pot: mi-ko wal--ni-ko di-on-a take the rice from the fire. (9.68)

de-tom-a, di-tom-a $v t$. lift or carry in the arms, esp. for heavy things. $(9.532)$

de-tom-brot-a vt. lift or carry something large and heavy. (9.532)

dek-dek-a vi. twitch, jerk, of a newlykilled animal. (9.322)

del-a vt. sting, as bees. (4.482)

del-ang $n$. small spirit house that is built in front of a house when someone dies. (8.58)

del-kom-tet-a $v t$. sting while curled up. (4.482)

del-ong-del-ong $a d v$. with big eyes, with open eyes. (9.42)

del-del-a vi. have the rough sound of a broken machine, a broken water pot; make insufficient noise, as a bad drum head; hoarse, as when one cannot speak or sing properly due to a cold, or from drinking too much rice beer: git-ok del--del-a throat is hoarse. (3.624) 
dem•-a, dem•-et-a $v t$. fold, of cloth, paper, etc.; bend, kneel. (9.641)

dem-dem-a, dem-dem-a $v t$. bounce a child in one's hands. (9.515)

den'-a vt. cut, chop, cut by striking blows. (9.573)

den-bak-bak-a $v t$. cut off bark. (9.573)

den-chek-chek-a $v t$. make small chops, here and there, as when chopping the nodes while preparing split bamboo. (9.573)

den-chrit-a vt. split into a few pieces, esp. of bamboo lengths. (9.573)

den-drak-a $v t$. chop into many pieces. (9.573)

den-jem-jem-a $v t$. cut into small pieces. (9.573)

den-kil-ot-a $v t$. make small cuts or chips with the tip of an at-te (bamboo knife). (9.573)

den-kim-bak-a vt. cut partway through. (9.573)

den-kip-a vt. bang the point of an at-te (bamboo knife) into something like a tree in order to hold it when resting. (9.573)

den-ku-rak-a vt. split into two halves, esp. of lengths of bamboo. (9.573) den-mit-chi-a $v t$. cut into small pieces, of wood, etc. (9.573)

den'-pret-a vi. chop into two pieces, chop open, as a coconut; split bamboo in half lengthwise. (9.573)

den-pri-a, den-pri-pri-a vt. cut into lots of small pieces, of meat, wood, branches, etc. (9.573)

den'-pri-den'-mit-chi-a $v t$. cut into lots of pieces. (9.573)

den-pru-a vt. cut a hole through. (9.573)

den-sot-a $v t$. dig, as a channel for water to flow. (9.576)

den-tak-tak-a vt. chop many little cuts into something with a bamboo knife. (9.573)

den-tong-a vt. cut across, cut through, cut into lengths, of a log or stick. $(9.573)$

den'-wak-a vt. cut a lot, cut through. (9.573)

den-wat-a vt. dig, as a water channel to let water through. (9.576)

deng-deng-a vi. squirm as one might try to escape capture; be restless, wiggle the way a small child wiggles, jump around; flail with limbs. (9.319)

deng-ga $n$. a red spinach-like vegetable of which the leaves are eaten, larger than lal-sak. $<\mathrm{B}(5.23)$

deng-gok-a vi. bow deeply, bend over, of people; with the head bent down; let the head flop when falling asleep; curl up with knees drawn up and face down; bent over, of rice plants. (9.348)

deng-gu $n$. bad person, naughty person; often used affectionately for children, rascal. (8.33)

-dep- aa. flat, flatten: pel-dep-a flat; chi-dep-a flat, of noses; su-dep-a caught or pinched, pounded into a bamboo tube; $\mathbf{k u} \cdot \mathbf{- d e p - a}$ flat mouth; rim-dep-a push with a finger to test ripeness of fruit. (2.311)

des $n$. country, nation, land. $<\mathrm{B}(8.22)$ 
di-di $n$. female teacher, older sister, primarily a term of address. $<$ B (8.6) di-pang-pil-ek-a vi. upside down, as a person with feet in air and head on ground. (9.327)

di-sal-o-ni di-sal-o-na, in-di-sal-o-ni in-di-sal-o-na $l w$. from this time to that time, (said while pointing to two places in the sky marking where the sun would be at the times referred to). (2.52)

di'-a, de'-a vt. lift up, carry, (see de•- for compounds). (9.532)

di-sa, pi-sa $n$. child. (6.822)

diin $n$. day, today. $<\mathrm{B}(2.53)$

-dik- aa. ugly, unpleasant. dong-dik-a in a bad condition, as having a dirty body, uncomfortable; ni-dik-a ugly to see; kin-a-dik-a ugly to hear. (3.457)

dik- $c p$. categorizing prefix for pots: dik-tom rice beer brewing pot; dikte smallish pot for water, etc. -dik $d n s$. pots: me-dik rice pot; sam-dik curry pot. (0.17)

dik-a vi. thick, tight together, of growing things, plants, hair: kim-il dik-a thick body hair. (2.25)

dik-dik-prak $a d v$. for a short time. (2.53)

dik-dik-sa, dik-dik-ha $a d v$. for a little while, a short time, for just a moment. (2.53)

dik-im-dik-im $a d v$. slowly, ponderously, as elephants walk. (2.724)

dik-ka $n$. an earthenware pot together with its cane sling that is used for brewing rice beer. (5.432)

dik-kil-eng $n$. broken pieces of pottery. (5.432)

dik-ku-li $n$. a very small earthenware pot, smaller even than a dik-te pi-sa. (5.432)

dik-te, dik-ti $n$. smallish pot, often but not necessarily earthenware, for water, etc., smaller than a gim-bi. (5.432)

dik-te pi-sa, dik-ti pi-sa $n$. small pot. (5.432)

dik-te sa-rong $n$. middle sized pot, larger than dik-ti pi-sa. (5.432)

dik-tom $n$. large round open mouthed earthenware pot, used for brewing rice beer. Does not include a cane sling. (5.432)

-dil dns. long and thin: ja'-dil root; bi-dil vine. (2.312)

dil-a vi. poor, impoverished. (8.441)

dil-a vt. guide, lead, show how. -dil- $a a$. lead, guide, show the way, cause: cha-dil-a feed; ken-dil-a frighten; ha-bu-dil-a give someone a bath; dong-dil-a care for a baby or small child. (9.73)

dil-dek-ga-dek $a d v$. swing, wobble, move back and forth, of a bamboo bridge, loose post, etc. (9.258)

dil-ding bal--jak $a d v$. become an adolescent, mature. (6.78)

dil-e re'-a, kim-e chu-a $v p h$. a way of describing a husband and wife as a couple, (lit. lead in walking, sleep as married). (6.657) 
dil-gang-gil-a $v i$. very poor, without anything. (8.441)

dil-ing-dil-ing $a d v$. swirling about, as dust. (4.248)

dil-nok-kol-a vi. very poor, without anything. (8.441)

dil-rik-rik-a vi. grow poor. (8.441)

dil-tek-tek-a vi. very poor, without anything. (8.441)

dil-dek-ga-dek $a d v$. loose, of a tool handle, post, etc. (2.23)

dil-dil-a vi. tremble, shake, from cold, fear, anger. (9.322)

dil-dil-kep-kep adv. tremble with cold or fright. (9.322)

dil--dok-dil-dok $a d v$. very soft, like butter, curds, European bread. (3.664)

dil·-gep-dil-chi $a d v$. short and fat, of people. (6.48)

dil--mik-dil'-mik adv. smoky. (5.422)

dim-a vi. swarm, settle down in large numbers, of insects, etc. (4.486)

dim-breng-a vi. straight, direct, of roads, a house wall; in line, like areca palms; stand erect; having a direct and clear manner of speaking. dim-breng-dim-breng $a d v$. (2.318)

dim-breng-chot-chot $a d v$. very straight, as a house wall or pineapple rows. (2.318)

dim-dak, dim-dak-in $n$. every, all. (3.343)

dim-dim-a vi. to make a sound, of drums. (8.83)

dim-dim-a, wal dim-dim-a vi. burn vigorously, brightly. (5.425)

dim-il-a adv. dim, not bright: dim-il-e ching-a shine dimly. (3.558)

dim-prong-dim-prong $a d v$. smoky, dusty, swirling with a lot of smoke, blow up in dust. (5.422)

dim-a vt. make a fence or partition; build walls (be-ra) of bamboo, thatch, sam-sim-a-ri stalks, or mud; hang curtains. (5.631)

dim•-a, dim•-dim-a vi. damp, slightly wet. dim•-e-dim•-e $a d v$. (9.272)

dim-ak $n$. soot, as on the bottom of a cooking pot. (5.422)

dim-chang-a vt. put up a mat partition, in a house, across a path. (5.631)

-ding dns. long and thin: kil-ding thread; wa'-ding thin, flexible bamboo tie strips; ri-ding pole for holding cloth to dry. ding- cls. classifier for long thin things: hairs, cords, strings, pieces of cane, wire, roads, etc. (2.312)

ding-a vt. attack, fight, wage war, snarl in defiance. (8.36)

ding-chon-a $v i$. thin, of long things, string, vines. (2.211)

ding-dal-a vi. big around, of long things, string, rope, vines. (2.211)

ding-dang $a d v$. different: ding-dang-ding-dang $a d v$. different from each other, (A•chik: ding-tang). (3.435)

ding-dang-ga-dang $a d v$. separately, doing things in different ways, disorderly. (3.435)

ding-de-a, ding-di-a, ding-de-ding-de vi. hang by a hook, strap, etc. $(9.255)$ 
ding-ding adv. tightly stretched, without wrinkles, of cloth, string. (9.642)

ding-grik-a vi. echo in dak-grik-a ding-grik-a fight, threaten, wage war. (8.36)

ding-rak-a vi. strong, of long thin things, string, jute, etc. (3.72)

ding-si-si $a d v$. thin, of thread. (2.211)

ding-sreng-a $v i$. thin, of string, wire, bamboo strips, etc. ding-srengsreng $a d v .(2.211)$

ding-sri-sri, ding-si-si, ding-sri-mik-mik, ding-si-mik-mik adv. thin, of string, wire, etc. (2.211)

ding:-a vi. hot. (3.673)

ding-bil-a vi. to be hot personally, become hot, of people. (3.673)

ding-bru-ding-bru adv. a bit warm, of people. (3.673)

ding-chik-il-a vi. burn very strongly. (5.425)

ding-chip-a vi. very hot. (3.673)

ding-ding $a d v$. while hot, as for food that is hot for eating. (3.673)

ding-du-a vi. have a fever, feel hot. (8.93)

ding-gek-a $v i$. very hot, as a very hot day, very hot water. (3.673)

ding--il-a ding-cha-a vi. burning on skin from touching chilies, (same as si-sa-a). (8.94)

ding-ka-ri $n$. hot season. (2.84)

ding-nik-a, ding-e nik-a vi. find it hot, seem hot. (3.673)

ding-rit-a vi. somewhat hot, of people. (3.673)

-dip- $a a$. pause: dong-dip-a stop, pause; cha-deng-dip-a stand for a bit, pause while walking; na-dip-a stop hearing for a bit. (9.14)

dip-a $n$. bed bug. (4.468)

dip-at, dip-et, stip-at, stip-et $p p$. 1. since, (follows augmented locative -o-ni): me-ja-o-ni dip-at since yesterday; jak be'-o-ni dip-at gam rim•-na man-ja-ing-jok I haven't been able to work since breaking my arm. 2. until, up to, as long as, (follows augmented locative -o-na): ambin-o-na dip-at until tomorrow; chu-ti-o-na dip-at until the holidays; man-a dip-at as much as possible. (1.12)

dip-dip-ing $n$. wooden base for a pot. (5.432)

dip-ik-a vi. powdery and dry, very fine. dip-ik-dip-ik $a d v$. (3.668)

dip-il $n$. fence around a sam-ba-si-a (altar). (8.58)

dip-il-ok $n$. frog-like animal that lives on land, even in people's houses, and that is seen and heard in the rainy season. (4.447)

dip-it-a vi. tough, of wood that doesn't break when chopped, of wood that is a bit green and flexible; tough to chew, like beef, chewing gum. (3.668)

dip-u dip-u $a d v$. very soft. (3.664)

dis-mel, dis-mil $n$. decimal, 1/100 acre, smallest unit of land, (same as ko-ra), 435.6 sq ft. $<$ E (5.12) 
dit-a vt. filter water through ashes to obtain soda; filled with liquid, as water behind a barrier, breasts filled with milk. (5.458)

do be-en $n p h$. chicken meet. (5.401)

-do-, -du- aa. up, upward: ga-do-a climb; sal-do-a pull up, as in drawing water; bil-do-a fly up; chrok-do-a jump up; chil-do-a lift up onto the head or shoulders. (2.14)

do-a, du-a vt. climb, go up: ha--bri-ko do-a climb the mountain. -do-, -du- $a a$. up, upward: ga-do-a climb; climb up, as a tree; get on, as a bus.; sal-do-a pull up, as in drawing water; bil-do-a fly up; chrok-do-a jump up; chil-do-a lift up onto the head or shoulders. (9.318)

do-ba- cls. classifier for rice fields, area surrounded by a dike. (1.38)

do-kan, do-han, du-kan, du-han $n$. shop. $<\mathrm{B}(8.449)$

do-nu $n$. holder for the arrow in a crossbow. $<\mathrm{B}(5.845)$

do-ra- cls. classifier for unit of weight equal to 5 seers, about five kilos. $<\mathrm{B}(1.36)$

do-ri $n$. string, rope, esp. jute rope. $<\mathrm{B}(6.594)$

do-rik-rik-a, du-rik-rik-a, do-ry-rik-a vi. climb to the top, climb up and up. (9.318)

do-ro-a, du-ru-a vi. sing a kind of competitive chant. (8.83)

do*-, do•-o, do, du•-, $\mathbf{d u} \cdot \mathbf{u}, \mathbf{d u} n$. bird; specifically: domestic fowl, more broadly any bird at all. Combining form: do*-, du*-. cp. prefix for varieties of birds: do'-pi-sa chick; do-bi-ma hen, female bird; do•-bi-pa cock, rooster, male bird. (5.33)

do-a-kin-ti, du-a-kin-ti $n$. a small bird that lives in trees. (4.43)

do-a-mik, du'-a-mik $n$. ash-colored bird that lives in holes in the ground and that gives a message to people (me-me-me) before kinsmen arrive. $(4.43)$

do-ang, du-ang $n$. black faced eagle-like bird that associates with me'-mang (ghosts) and whose cry is a warning of death. (4.43)

do-bak $n$. bat, the flying mammal. (4.43)

do-bi-ma, du'-bi-ma $n$. hen, female bird. (4.43)

do-bi-pa, du-bi-pa $n$. cock, rooster, male bird. (4.43)

do-bik, du-bik, do-bi-bik $n$. bird's intestines, used in divination. (4.471)

do-bik ni-a, du'-bik ni-a vt. inspect the fowl's intestines for omens, esp. the inspection for omens that is a central part of a marriage ceremony. $(8.562)$

do-bil-dok, du•-bil-dok $n$. a bright green bird, edible, (blue throated barbet), (Beng. tu-kur). (4.43)

do-bit-bit, du'-bit-bit $n$. bird that is larger than a dove and somewhat black, (Beng. bul-bul paki). (4.43)

do•-bit-chi, du·-bit-chi, do•-chi, du·-chi $n$. egg, bird's egg. (4.475) 
do-bring, du-bring $n$. birds of the forest, wild fowl. (4.40) do-bu-buk $n$. a kind of bird, (crow-pheasant). (4.43)

do-chi, do--bit-chi $n$. egg, bird's egg. (4.475)

do-chi-dik, du-chi-dik $n$. bird coop, chicken coop, a place to keep a domestic fowl, made of earth or basketry. (5.852)

do-chi-pu-ra $n$. omelet-like dish made with eggs and rice flour. (5.409)

do-chi-rong, du-chi-rong $n$. a turmeric colored bird the size of dove, that builds its nest in fork of tree branches, (golden oriole). (4.43)

do'-chok, du'-chok $n$. a small bird, the size of a chan-cho-ra, with pointed back end, that builds hanging and swinging nests in trees, (Beng. ba-bui pa-ki). (4.43)

do'-chong-dang, du'-chong-dang $n$. a long-legged ground-living bird slightly smaller than a do-grik. (4.43)

do'-di, du'-di $n$. peacock, (Beng. moi-ur). (4.43)

do-ding-di, du'-ding-di $n$. a small dark blue bird the size of a chancho-ra that builds a small hanging nest, (purple sun-bird). (4.43)

do-dok-a, du·-dok-a $v t$. kill a domestic fowl; name given to the traditional marriage ceremony at which a chicken sacrifice is the central event, and where the intestines are inspected for omens. (8.562)

do·-ga, du·-ga $n$. door, especially a bamboo lattice door. (5.638)

do-ga-chol, du-ga-chol $n$. doorway. (5.638)

do-gep, du-gep, do'-gep-gep $n$. duck. (4.43)

do'-grik, du·-grik $n$. large ground-dwelling bird, with 'grik' as its call. (4.43)

do'-gring, du·-gring $n$. a small basket in which domestic fowl can nest, or in which they can be carried to market. (5.852)

do'-gu-gu, du'-gu-gu n. wild pigeon, dove, (Beng. ko-bu-tor). (4.43)

do-ja-rak, du-ja-rak $n$. duck, (same as do--gep). (4.43)

do-ja-rak gang-si, du-ja-rak gang-si $n$. a type of duck that is larger than an ordinary do-ja-rak. (4.43)

do-jeng-gim, du'-jeng-gim $n$. a bird the size of a do-ma-sik-i that lives in groups. (4.43)

do-jil-ma, du'-jil-ma $n$. a common bird that comes in big flocks, a bit black and the size of $\mathbf{d u} \cdot-\mathbf{k u}-\mathbf{r u}$ pigeon. (4.43)

do'-ka, du·-ka n. crow. (4.43)

do-ka-si $n$. a kind of bird, (grey junglefowl). (4.43)

do-ka-sim-ek, du-ka-sa-mek $n$. a kind of crow, smaller than a do-ka, (Beng. chu-to-ka, dar-ka). (4.43)

do'-ki, du'-ki, du'-ni ke'-em-a $n$. bird droppings. (4.475)

do'-ku-ru, do'-kru, du'-ku-ru $n$. pigeon, dove, joking term for ri-gong penis. (4.43)

do-ku-ru-kal-dek $n$. smaller variety of dove. (4.43) 
do-ku-ru-rong-ru $n$. larger variety of dove. (4.43)

do-ku-ru-wa'-sin $n$. a kind of dove, (emerald or bronze-winged dove). (4.43)

do•-ma, du•-ma $n$. a long tailed bird, (same as du•-pa-jong-gol). (4.43)

do'-ma-sek-i, du'-ma-sek-i $n$. a bird, larger than chan-cho-ra, with white and black on its wings that is more visible when flying; the bird pictured on the two taka Bangladeshi note, (Beng. du-el). (4.43)

do-man-di $n$. domestic fowl. (4.43)

do-mat, $\mathbf{d u} \cdot \mathbf{- m a t} n$. animals generally, both wild and domestic, (lit. birds and squirrels). (4.40)

do'-mi-sel, du•-mi-sel, do*-mi-sal $n$. junglefoul, kind of bird in which female has a short tail, male a long one, (Beng. bon-mu-ru). (4.43)

do'-mok, du•-mok $n$. goat, (A•chik: do•-bok). (5.35)

do-mok an-dra $n$. uncastrated male goat. (5.35)

do'-mok-me-ra, do•-mok-be-ra $n$. sheep. (5.35)

do'-pa-jong-gol, du·-pa-jong-gol $n$. bird with a long black tail, (piedcrested cuckoo). (4.43)

do-pat-chi, du-pat-chi $n$. swallow, a chan-chora sized bird that flies in circles. (4.43)

do•-pi-sa, du·-pi-sa $n$. chick, baby bird. (4.43)

do-pin-chep, du•-pin-chep $n$. sparrow like bird with nests made from curled up leaves, yellow, with a call sounding like 'chep-chep-chep', (babbler). (4.43)

do-pit, du'-pit $n$. a small bird, similar to a chan-cho-ra, (spotted myna). (4.43)

do'-po, du•-pu $n$. owl, (A•chik). (4.43)

do-po-mik-del-ong $n$. a large-eyed owl. (4.43)

do-po-tot-ting, du-pu-tot-ting $n$. large black owl that lives in trees, (Beng. pe-sa). (4.43)

do-ra-song, du-ra-song $n$. the bird that is killed in order to inspect the intestines for omens, specifically the bird sacrificed in a marriage ceremony. (8.58)

do-rang-ding $n$. a small yellow bird, the size of a chan-cho-ra, that flies in high circles. (4.43)

do-reng, du'-reng $n$. hawk, kite, a big black bird that soars and then swoops down to get meat, (Beng. chiil). (4.43)

do-reng-chi-jeng $n$. smallest of three kinds of hawks. (4.43)

do-reng-gan-tong $n$. largest of three kinds of hawks. (4.43)

do-reng-gang-pak $n$. plant that grows in trees, with big, long, and strongly toothed leaves. (4.656)

do-reng-se-na $n$. a reddish-colored hawk. (4.43)

do-reng-wal-gu-si, du-reng-wal-gu-si $n$. middle-sized of three kinds of hawks. (4.43) 
do-rim-it $n$. a kind of bird, (chestnut bellied nuthatch). (4.43)

do'-sat-til a-sek-ki, du'-sat-til a-sek-ki $n$. the constellation of the Pleiades. (4.212)

do'-sik, du-sik $n$. parakeet that can be trained to talk, with curved red beak and long tail, larger than a do-ma-sek-i. Makes holes in trees, (Beng. ti-a pa-ki). (4.43)

do-sil-ek $n$. an insulting name, implying that someone has no modesty. (7.27)

do·-stil, du'-stil $n$. egg yolk. (4.475)

do-tek-mi $n$. plant growing on tree branches with long, thin, almost needle like $3-5 \mathrm{~cm}$. leaves and red to orange flowers but no roots, (Beng. por-ga-cha). (4.656)

do•-til-eng, du•til-eng $n$. woodpecker, red, yellow and some black, a bit smaller than a dove. (4.43)

do-tip, du'-tip $n$. a very small bird. (4.43)

-dok num. six, (A•chik). (3.3706)

dok-a vt. beat, knock, hit, tap, beat drum; make, forge iron tools; create, as a god creates people. (9.553)

dok-a sik-a vt. play musical instruments, (lit. beat and blow). (8.83)

dok-a-sik-a-ni $n$. musical instruments, (lit. things beaten and blown). $(8.85)$

dok-ak-a vt. hit forcefully, with hand or stick. (9.553)

dok-ban-da, dok-man-da $n$. a Garo woman's wrap around skirt cloth of traditional design. (6.526)

dok-bo, dok-bu, git-ok-bu $n$. goiter, (lit. neck-swelling). (8.956)

dok-chak-a $v t$. hit back, return a hit. (9.553)

dok-chap-a $v t$. hit at the same time, as the ball in football. (9.553)

dok-chap-grik-a $v t$. knock two things together. (9.553)

dok-chep-chep-a $v t$. hit repeatedly in order to break or damage. (9.553)

dok-chrak-a $v t$. knock with the hand or stick; hit and cause to move; knock down, as fruit from a tree: ki-me dok-chrak-a knock down, knock off with the tail. (9.553)

dok-chrak-a, ki-me-cha dok-chrak-a vt. knock down, knock off with the tail, as brushing off flies, of cows, buffaloes. (4.488)

dok-dek-a vi. waggle the head from side to side, a gesture of agreement. (9.41)

dok-dep-a, dok-dep-dep-a vt. beat flat, flatten, crush. (9.578)

dok-drak-a vt. knock open, as a door, knock out of the way. (9.553)

Dok-gip-a $n$. forger, maker of iron tools, used as a name for the Christian God. (8.54)

dok-gok-a vt. hit and separate, as to knock off a handle. (9.553)

dok-grik-a vi. fight, (lit. hit each other). (8.36) 
dok-jem-jem-a vt. hit continuously. (9.553)

dok-jol-jol-a, dok-jo-jol-a vt. hit one after another as one moves along. $(9.553)$

dok-kak-kak-a, kak-kak-e dok-a vt. tap with a knuckle, knock. (9.553)

dok-kam-a, dok-kam-kam-a vt. hit constantly. (9.553)

dok-kiin $n$. south. $<$ B (4.218)

dok-krep-a $v t$. break or crush by pounding. (9.578)

dok-nek-a vi. waggle the head from side to side but more slowly than dok-dek-a, turn the head to the side just once, a gesture of agreement. (9.41)

dok-pak-a vt. knock off, as fruit from a tree; shake off; slap off, brush off, as dirt with the hand; action of a woman's kinsmen when they take her back because they don't like her husband. (9.557)

dok-pil-deng-a vt. beat flat. (9.649)

dok-pil-dep-a $v t$. beat flat. (9.649)

dok-pret-a vt. hit hard, often with a stick or tool, in order to break something such as a coconut. (9.284)

dok-pri-a $v t$. hit and break into pieces, as bricks, earth. (9.284)

dok-prong-a $v t$. hit, slap, slap off dust with the hand or a cloth. (9.557)

dok-pru-a $v t$. knock a hole through. (9.553)

dok-rip-ak-a $v t$. hit everything. (9.553)

dok-ro-a vi. tall, of trees, bamboo, people. (2.213)

dok-ro-rek-a, ki-me dok-ro-rek-a vt. wag tail, move tail back and forth, of cows, dogs, cats. (4.488)

dok-rok-a $v t$. knock off, knock one thing against another, as to clear dirt from a basket; slap dirt off; brush off, as a cow brushes off flies with its tail. (9.557)

dok-sa-ri $n$. wrap around skirt, simpler than a dok-ban-da. (6.526)

dok-sap-a $v t$. slap off with a cloth. (9.557)

dok-sik-a-a vt. return a hit, hit back. (9.553)

dok-sim-sim-a $v t$. strike a lot, repeatedly. (9.553)

dok-sit-tap-a $v t$. hit hard, as to flail clothes against something hard when washing them. (6.57)

dok-srap-a vt. knock off, brush off, as a person might flick off dirt with a clocth. (4.488)

dok-srap-a, dok-sap-a vt. hit hair, slap hair, when washing it. (6.57)

dok-srok-a vt. hit lightly, as an older child hits a younger child, as cobwebs are hit with a broom. (9.553)

dok-tap-a, dok-tep-a $v t$. pat with an open palm. (9.552)

dok-tat-a $v t$. beat forcefully, beat to death. (9.553)

dok-ting-a $v t$. tap, knock, especially something that resounds, as to test the soundness of a pot; knock on a door. (9.552) 
dok-tip-tip-a $v t$. slap an opening, as to cover someone's mouth to prevent him from speaking; slap an ear; pat, slap several times: pat a child to soothe him; slap a basket to knock things into place. (9.552)

dok-tok-tok-a $v t$. clean off body with repeated slapping; knock something such as a basket to loosen the dirt inside; tap, knock, as on a door; knock a $\log$ to see if it is hard; knock with something held in hand, like a stick. (9.557)

dol- cls. classifier for sides, teams, groups of people. $<$ B (8.48)

dol-deng-dol-deng, dol-deng-ga-deng adv. hanging, dangling, swinging. (9.257)

dol-dop-a vi. sag, of a string, cloth cradle; opposite of kring-a. (9.642)

dol-ong $n$. masonry bridge. (5.646)

dol-a vi. make insufficient noise, of a drum. (3.626)

dol-a vt. roll up. dol-- cls. classifier for rolled-up bundles of banana leaves or other leaves to be used to eat from. (9.645)

dol-ok $a d v$. fall slowly or gently, without damage or injury. (9.326)

dom $n$. Hindu caste that raises and sells pigs. $<$ B (6.89)

dom-kol-os $n$. little white-flowered bitter-tasting inedible wild herb, probably a mint. $<$ B (4.656)

dom-kol-os-git-chak $n$. an herb with white and pale purple flowers. (4.656)

don-a vt. call, name, give a personal name: ang-ko Ming-ji don-a I am named Mingji, (lit: 'Ming-ji' names me). (7.18)

don-a vt. put, place, put aside, omit. (9.68)

don-a, nok-na don-a vt. give one's nok-na (heiress daughter) to the man who becomes one's nok-rom (son-in-law and heir). (6.651)

don-ba-a $v t$. leave behind. (9.538)

don-bek-a $v t$. leave something with someone whether or not it is wanted, as to leave someone with a crying child; leave something carelessly, without permission. (9.68)

don-chak-a vt. put in, keep in for storage, as things in a trunk, salt in a jar; shut up in, as a bird in a cage. don-chak-gip-a. $n$. holder, container, storage place, place to put something. (9.68)

don-dap-a $v t$. put on top of. (9.68)

don-e $a d v$. leaving behind, leaving out, omitting: sal-sa don-e min-no-a will be ripe in two days, (lit. omitting one day). (9.538)

don-gal-a, don-gal-gal-a, don-ga-gal-a vt. put down carelessly. (9.68)

don-gu-al-a vt. forget where one has put something. (9.538)

don-jri-a $v t$. rename: bi-ming-ko don-jri-a give a new name. (7.18)

don-pa-a $v t$. put together. (9.68)

don-ru-ra'-a vt. move, change something's position, move things back and forth. (9.511) 
don-sam-a vt. echo in don-u-a don-sam-a hide. (9.344)

don-u-a $v t$. hide, conceal. don-u-a ku-pa-a vt. not say everything, hold some things back. (9.344)

dong-a $v i$. there is, there are; have, belong; be at, reside, dwell; be married to. $(9.11)$

dong-ba-a vi. stay for a while, stop while passing. (9.11)

dong-bing-bang-a vi. exist in a confused, mixed up way. (9.11)

dong-chak-a vi. stay at temporarily, live at for a while, dwell at, reside. (9.11)

dong-chap-a vt. add a wife; attach, join, put beside; pile up, as leaves, cloth, paper, give a second wife to a man. (6.652)

dong-cheng-a vi. be somewhere first, stay first. (9.11)

dong-chik-a vi. endure. (7.56)

dong-chip-chip-a vi. endure, remain quiet. (7.56)

dong-dam $n$. living place. (5.644)

dong-dam-a vi. be in a place, dwell. (9.11)

dong-dang-a $v i$. be at a place regularly; stay, dwell; often negative: dong-dang-ja too busy or restless to stay in the same place, always moving. (9.11)

dong-dik-a vi. in a bad condition, as having a dirty body; uncomfortable. (3.457)

dong-dil-a $v t$. care for, look after a child, kinsman: gend-a-ko dongdil-a care for the baby. (9.73)

dong-dip-a vi. stop, pause. (9.14)

dong-e $a d v$. leaving out, skipping, omitting: am-bin-o dong-e skipping tomorrow. (3.346)

dong-e-dong-e $a d v$. now and then, at intervals. (2.71)

dong-gip-eng dong-gru $n$. the stars of Orion's belt and sword, (lit. being across, being long). (4.212)

dong-kam-a, dong-kam-kam-a vi. reside, live for a long time at, stay permanently; for a long time, of rain, sleep, etc. (2.53)

dong-kin-ap-a vi. in a good state, comfortable. (3.455)

dong-pak-a vi. stop on the way. (9.11)

dong-peng-a $v t$. obstruct, block with one's presence. (9.77)

dong-rik-a vi. stay, stay behind, be pregnant. (9.11)

dong-rim-a vi. be peaceful, without quarrelling. (8.32)

dong-ru-rak-a, dong-ru-rak-a dong-su-sak-a vi. restless, active, can't sit still. (9.324)

dong-ru-ru-a vi. be some place for a long time in idleness. (9.11)

dong-sak-sak adv. have a lot of possessions, well off: dong-sak-sak cha--ing-a eating well. (3.342)

dong-si-a vi. echo of dong-dik-a in a bad state, uncomfortable. (3.457) 
dong-tim-a vt. wait, stay and watch; guard, look after. (9.73)

dong-tim-gip-a $n$. defender, guardian, one who watches. (9.73)

dong-wil-wil-a vt. surround, as people stand around someone or something. (9.11)

dor-mo $n$. religion, denomination. $<\mathrm{B}(8.51)$

dot- cls. classifier for posts, lengths of bamboo, stalks, unsplit pieces of firewood, rice seedlings, etc. (1.32)

dot-mi, dot-ni $n$. type of lizard, about $20 \mathrm{~cm}$. long, that lives in holes in trees and says, 'kak-ku', or, 'dot-me'. (4.441)

dot-ni-ja'-pa-bi-du $n$. a tree-climbing vine. (4.655)

dra-e $a d v$. forcefully, against another's will, without permission. (7.56)

-drak- aa. break apart, separate, peel off: pek-drak-a spread apart, of legs or toes; o-drak-a peel, remove peel, skin; jot-drak-a poke and tear off, of paper, cloth; dok-drak-a knock out of the way; bit-drak-a spread slightly apart, as thatch, clothing; pit-drak-a split, as firewood. (9.536)

-drak- $a a$. strongly: ring-drak-a drink a lot; wa-drak-a rain heavily; mik-u-drak-a make a loud noise; ring-drak-a sing loudly. (3.72)

drak-a vi. badly torn, in tatters, of cloth, fences, mats, baskets. -drakaa. break apart, separate, peel off: pek-drak-a spread apart, of legs or toes; o-drak-a peel, remove peel, skin; jot-drak-a poke and tear off, of paper, cloth; dok-drak-a knock out of the way; bit-drak-a spread slightly apart, as thatch, clothing; pit-drak-a split, as firewood. (9.292)

-drang ns. plural marker, synonym of -rang: bol-pang-drang trees. $(0.11)$

dren $n$. small constructed irrigation channel. $<\mathrm{B}(5.114)$

drin-a vi. slide down, break down; collapse, of earth, of wells, river banks, house walls. (9.212)

drip-drip $a d v$. following one another, one by one or in groups, of people or animals. (9.328)

drok-a vi. cluck of a hen when calling her chicks. (4.476)

du-a $v t$. give birth, of animals: wak pi-sa-ko du-a give birth to piglets. (6.77)

du-a, chi du-a vi. to flood. (4.33)

du-ait, du-at, du-et $n$. oil lamp. $<$ B (5.82)

du-bil-a $n$. a kind of short grass that is eaten by cows and goats and is useful for stopping blood. $<\mathrm{B}$ (4.652)

$\mathbf{d u}^{*}-\mathbf{d} \mathbf{d u}, \mathbf{d u}^{*} \mathbf{- u}, \mathbf{d o}^{*}-\mathbf{n}$. bird, do*- or $\mathbf{d u}^{*}-$ are the forms that takes suffixes, (see do- for compounds). (4.43)

$\mathbf{d u} \cdot \mathbf{a} n$. a thin flexible strip of bamboo, one rougher kind is used for tying by twisting, the other finer kind for weaving into a basket. (5.854)

$\mathbf{d u} \cdot \mathbf{- a} v t$. feed solid food, such as rice, to a child or invalid; feed at a festival by putting food directly in someone's mouth; feed domestic animals, bait birds. (5.478) 
$\mathbf{d u} \cdot$-dap $n$. raised earthen platform forming a fireplace in an old style house. (5.636)

du·-gek-gek-a $v t$. feed by force. (5.478)

du-gring, do-gring $n$. a small basket in which domestic fowl can nest, or in which they can be carried to market. (5.852)

du-ja-gip-i $n$. a meter tall shrub with laurel-like leaves which are first warmed in fire and then pressed onto the skin medicinally. (4.64)

du-ri-si $n$. a kind of rang (gong). (8.85)

du'-sim-a-bi-du, du'-jong-ma $n$. large and very long vine, up to $5 \mathrm{~cm}$. in diameter and with deep longitudinal cuts in its bark, with edible sour tips. (4.655)

duk ong-chak-a vph. sympathize, feel grief for. (7.741)

dul-a n. dust. $<$ B (4.248)

dul-i $n$. plain baskets of varied size used for storing rice. $<$ B $(5.852)$

dup-dup, dip-dip $a d v$. noise of a banging rice pounder. (3.624)

dut $n$. cow's milk. $<\mathrm{B}(5.409)$

-e, -i pvs. suffix that subordinates one verb or clause to the one that follows; having, being: ang-a nok nap-e, a-song-jok having entered the house, I sat down. (0.216)

e-jam-a, i-jam-a, hi-jam-a, hi-jam-jam-a vi. yawn. (7.96)

e-kor $n$. acre, unit of land measure, 100 decimals. $<$ B (5.12)

-e-min-a, -i-min-a, -e-ming pvs. subordinating suffix, elaborated equivalents of -e, having, being: cha-e-min-a, ang-a ok-ka-jok having eaten, I'm full. (0.216)

-e-ming, -i-ming, -e-min-a pvs. subordinating suffix, elaborated equivalents of -e, having, being: man-de-na ken-e-ming, kom-e dong-a being afraid of the man, (he) is cowering. (0.216)

e-ren-chi $n$. a small edible fungus that grows on trees, distinct from bol-na-gil. (4.656)

e-ching, $\mathbf{i} \cdot$-ching $n$. ginger. (5.23)

e•-chu, $\mathbf{i} \cdot-\mathbf{c h u} n$. tip, end portion of a banana leaf, used in some traditional ritual offerings. e'-chu an-a, i-chu an-a vph. spread out the end portion of a banana leaf (at the base of an altar as part of a sacrifice). (4.675)

e·-gong, i-gong $n$. midrib of a banana leaf. (4.675)

e•-king, i-king, hi-king, re`-king $n$. woman's skirt, wrap-around skirt. (6.526)

e•-sal, i•-sal, re•-sal, hi•-sal $n$. banana leaf. (4.675)

el-a-chi $n$. cardamom. $<$ B (5.405)

el-di, hel-di, ol-di, hol-di $n$. turmeric. $<$ B (5.405)

el-du-el-du dak-a $v p h$. take deep breaths; gasp, as when sick. (9.45)

el-ek-ka $n$. an administrative district. $<\mathrm{B}(8.22)$

el-ep-a, hel-ep-a vi. lightning. (4.344) 
el-ok-a vi. echo in bu-su-a el-ok-a cough. (9.45)

el-u $n$. biting water leech, a few inches long. (4.466)

el-bu-a, hel-bu-a vt. spit out food, vomit a small amount, especially of a baby spitting up milk. (5.478)

em-ne $a d v$. for no reason; for practice rather than real, just happens. $<\mathrm{B}$ (3.44)

eng-a vt. untie, untied. (9.65)

eng-a, heng-a $v t$. threaten to hit. (8.36)

eng-ki-a vi. not like to, escape doing, dislike, avoid: kam-na eng-ki-a not like to work. (7.744)

-et pvs. imperative: dak-et do it. (0.215)

-et-, -it- $a a$. causative: nam-et-a make good, improve; chip-et-a close, cause to be closed, $(\mathrm{A} \cdot$ chik: -at-. (1.13)

et-a vi. wipe away or push away in order to dispose of, esp. of mucus from the nose, but also sticks from a fire. et-e gal-a wipe and throw away. (9.44)

et-et-a vi. tight, squeezed; unable to pass through, as an arm into a bracelet, a cork into a bottle, etc. (2.22)

ga-a vi. climb up, as a hill or tree; work, function, of an object such as a pen; have effect, comes, is present, as in ma-rang ga-a pollution, bad omen, under taboo. (9.318)

ga-a, han-o chi ga-a vph. swell up, of the body. (8.956)

ga-ak-a vi. crow, the cry of a crow. (4.476)

ga-bil-a $n$. big open bowl, of pottery or metal. $<$ B (5.432)

ga-chek $n$. small basket for onions, chilies, etc. (5.852)

ga-dil-a, ga-del-a $n$. cloud: ga-dil-a gri cloudless. (4.323)

ga-do-a, ga-du-a vi. climb, climb up, as a tree; get on, as a bus. (9.318) ga-do-rik-rik-a vi. climb to the top. (9.318)

ga-dom $a d v$. stage of maturation when fruit is filling out, riper than ga-stu but not yet bri-a. (4.692)

ga-dom-a vi. echo in me-li-a ga-dom-a get along well, without quarrelling. $<$ B $(0.5)$

ga-gak $n$. domestic duck. (5.35)

ga-gak-gang-si $n$. goose. (5.35)

ga-gu-ri, ga-gri $n$. girl's underpants. $<\mathrm{B}(6.526)$

ga-hat-a, ga-kat-a vi. climb; get on, as a bus; ride, as a bicycle, bus, etc. (9.318)

ga-jur $n$. carrot. $<\mathrm{B}(5.23)$

ga-kat-a, ga-hat-a vi. climb; get on, as a bus; ride, as a bicycle, bus, etc. (9.318)

ga-mi-pong $n$. altar for sacrifice. (8.58)

ga-mi-pong-ri-ding $n$. horizontal bamboo that is a part of an altar. $(8.58)$ 
ga-na $n$. blind. $<\mathrm{B}(8.92)$

ga-ra $n$. a small lizard with a red throat that lives in the forest. (4.441)

ga-ri $n$. cart, bus, auto, vehicle. $<\mathrm{B}(5.86)$

ga-rik-rik-a vi. climb to the top. (9.318)

ga-rim-ak $n$. layer of smoke and soot that collects on the ceiling. (5.422)

Ga-ro $n$. the word used by non-Garos to refer to those who more often refer to themselves as Man-de, Man-di or A·chik. (6.89)

ga-stu $n$. very early stage of maturation of fruit or vegetable when it is still very small, earlier than ga-dom. (4.692)

ga-wa beng $n$. a large kind of frog, (same as beng-bong). (4.447)

$\mathbf{g a}^{\cdot}-c p$. prefix for plant parts: $\mathbf{g a} \cdot \mathbf{- g o n g}$ stem of a leaf; ga-brong central part of a tree trunk or the tough central portion of some fruits; ga'-gittong stubble, stalks of plants. (0.17)

ga·-a vi. step; wear, put on, of shoes, socks. echo in nap-a ga•-a visit, (lit. enter step). (9.321)

ga:-ak-a vi. fall, fall down, of people or things; fall into, be a part of; count as kin, as belong in a kinship relation. -gak- $a a$. fall: gong-gak-a fall off, fall apart, of tools, posts; gak-gak-a flop, droop, like the head of a small baby. (9.326)

ga·-ak-chak-a vi. fall into: ha mi-o ga·-ak-chak-a, cha'-na nam-jajok dirt falls into the rice (so it is) no longer good to eat. (9.326)

ga·-ak-dap-a vi. fall onto, as a tree branch might fall on one's head, as bombs fall. (9.326)

ga-ak-ro-rot-a vi. leak or overflow, spill along the way as something is carried, of water, rice, etc. (2.22)

ga-ak-rong-rong-a vi. fall through. (2.22)

ga·-au-a vi. easily separated, flake off, of skin, bark; break apart readily; open up, of plant husks; not tough. (3.668)

ga-bat-a vt. step across; cross border. (9.321)

ga·-beng-bat-a vt. step over: am·-pok-ko ga-beng-bat-a step over the stool. (9.321)

ga·-brit-a vt. scrape back with feet, as a bird scrapes the ground when looking for food. (4.482)

ga-brok-a vt. step into, water, mud, dung; tread in, as one mixes water and dirt by walking in it, in order to make mud. (9.321)

ga-brong $n$. central part of a tree trunk; the tough central portions of some fruits (such as pineapple, jackfruit); the soft central portion of some kinds of sticks, pith. (4.673)

ga-brot-a vt. scrape with the foot, scrape with the foot in order to separate, as grain from the stalks; scrape the feet together. (9.321)

ga·-chak-a vt. fit, of the feet, as on a pedal: pe-dil-ko ja·-pa-cha ga-chak-e gu-rai-ing-a turning, fitting the feet on the peddle, (lit. fittingly turn the peddle with the feet). (2.22) 
ga-chep-chep-a vi. keep tramping on. (9.321)

ga'-chrak-a, ga·-chi-chrak-a vt. push back grain with feet like birds; scratch up dirt like dog. (4.482)

ga·-dap-a vi. step on, stand on. (9.321)

ga-deng-si-a vi. on tiptoe. (9.346)

ga·-gil-wak-a $v t$. get cut or injured by stepping on something. (9.321)

ga-git-ik-a vi. squirm, of baby; take action, move around briskly doing things; move limbs vigorously. (9.319)

ga-git-tong, ga·-tong $n$. stubble, stalks of plants left after harvest. (4.670)

ga-gong $n$. mid vein or stem of a leaf; stem of fruit or a flower; stem and midrib of a banana leaf; stubble of cut rice plants. (4.675)

ga•-grik-a vi. echo in nap-grik-a ga•-grik-a visit back and forth. (9.315)

ga·-jep-a, ga·-jep-jep-a vt. step with pressure; stamp on; walk in such a way as to drive fish into a net. (9.321)

ga·-jet-a ga·-jet-jet-a $v t$. grab tight, hold tight with legs or feet. (9.321) ga-jret-a $v t$. tie tightly on the foot, as shoelaces; step on hard. (9.65)

ga-kang $n$. flat rear part of an altar. (8.58)

ga-krep-a vt. step on and crush. (9.578)

ga'-min-ek-a vt. mix up, crush up with feet, as mud with water; step on and crush, as a cow steps on rice plants. (9.578)

ga-pin-ek-a vt. step on something and crush it, as cattle step on rice plants. (4.482)

ga'-ping-pak-a vi. push aside with foot, brush to the side, kick away. $(9.321)$

ga-pong $n$. handle of a tool, umbrella handle. (5.87)

ga-pret-a $v t$. burst or squash something by stamping on it. (9.321)

ga•-pru-a, ga•-pu-a vi. step into, as into a hole; step through. (9.321)

ga-ru-ru-a vt. stamp on with feet. (9.321)

ga'-srik-a vt. make a line with the foot; get cut by stepping on something sharp. (9.321)

ga'-srot-a vi. slip with the feet, scrape with the feet. (9.326)

ga-tek-a vt. stretch out a leg quickly, kick out once; kick by an animal, cow, goat, etc. (9.47)

ga-tek-tek-a vt. kick out vigorously and repeatedly, like an angry and crying baby. (9.321)

ga-teng $n$. insect's leg; stick or small branch that has been cut off. (4.472)

ga-teng, ga`-git-teng, ga-git-tong $n$. stick or small branch that has been cut off; manioc stalk that can be planted; stubble, stalks left after harvest; foot of an insect. (4.672)

ga·-tim-a vi. step with heel. (9.321)

ga-tim-a, chi ga-tim-a vi. fall, of water, water rushing downward, flow into a hole. (9.273) 
ga-tim-ra $n$. step to a house or veranda, edge of a veranda. (5.637)

ga-ting-a $v t$. kick lightly, kick a stationary object, stretch out a leg. (9.321)

ga-ting-dat-a vi. kick strongly, kick something so that it moves, as a football; kick or push with the foot. (9.321)

ga'-tong, ga'-git-ong $n$. stubble, stalks of plants left after harvest. $(4.670)$

gai-a $n$. a kind of short grass eaten by cows. (4.652)

-gak- $a a$. fall: gong-gak-a fall off, fall apart, of tools, posts; gak-gak-a flop, droop, like the head of a small baby, (cf. ga'-ak-). (9.326)

gak-a vt. peck, as a bird pecks at rice; nibble at, of people. (4.487)

gak-gak-a vi. flop backwards, droop, as the head of a small baby droops when carried in a cloth. (9.41)

gak-gil $n$. large tree with gray trunk, small round leaves and small inedible fruit, good for firewood, (Beng. si-riis). (4.632)

gak-jak-gak-si $a d v$. messy, of hair, uncombed. (6.58)

gak-u-a, gak-u tak-a, sko gak-u-a vi. nod, nod repeatedly, fall asleep so that the head nods; bend down. (9.41)

gal-a vt. throw away, dispose of; divorce; pour rice beer as an offering at a sacrifice. -gal- $a a$. take off, get rid of, throw away: sal-gal-a put away, as sleeping mat; king-gal-a uncover; su'-gal-a wash clothes, dishes, etc.; wak-gal-a vomit. (9.54)

gal-e-gal-e $a d v$. in part, omitting parts: gal-e-gal-e cha--a eat part, leave part. (3.347)

gal-ga-gal-a $a d v$. throw away carelessly, without waiting. (9.54)

-gal-gal-, -ga-gal- $a a$. quickly, carelessly, throw away: a-gan-gal-gala speak quickly; don-gal-gal-a put down quickly, carelessly; ak-tetga-gal-a pick quickly, carelessly, incompletely; pe·-gal-gal-a break and throw away. (2.726)

gal-grik-a $v t$. mutually dispose of, divorce each other. (6.655)

gal-o-rot-a, gal-u-rot-a vt. drop things along the way, as from the hand or from a torn sack. (9.54)

gal-wang- cls. classifier for small bunches of bananas, about eight fruit. $(1.34)$

gam, gam-u, kam $n$. work, labor, cultivation. (8.433)

gam gok-a, gam-u gok-a vph. hard-working, eager, enthusiastic. (7.725)

gam-a, gam-et-a, gro gam-a, gro gam-et-a, gam-a si-a vt. pay a fine, give money in compensation after a legal dispute. (8.15)

gam-ba-ri $n$. large tree with fairly large lozenge-shaped leaves. good heartwood and good for making drums, (Beng. jo-gin-i-chok-ro). (4.632) gam-cha $n$. small cloth, towel, napkin, loincloth, woman's top cloth, cloth worn on head, cloth used by little girls as a short skirt. $<\mathrm{B}(6.521)$ 
gam-chak-a vi. collect money from the participants in order to pay compensation in settlement of a dispute. (8.15)

gam-il-gam-il $a d v$. swelling on the body. (8.956)

gam-ra $n$. fork in a tree where branches divide. (4.671)

gam-a vi. make a noise, to sound, of thunder, drums, etc. (3.624)

gam•-ba $n$. $30 \mathrm{~cm}$. a weedy herb with ugly, crinkly, fuzzy, leaves and tiny white-purplish flowers; bad smelling, dies down in the rainy season. (4.656)

gam•-ba dal-gip-a $n$. an $8 \mathrm{~cm}$. weedy wild plant with smelly and fuzzy edible leaves. (4.656)

gam-ba-jak-ma $n$. plant with large lettuce-like leaves. (4.656)

gam•-ba-jak-sem $n$. little purple flowered inedible plant with small leaves. (4.656)

gam•-pret-a vi. sound loudly, of thunder, airplane, gun. (3.628)

gam•-ru-ru-a vi. make much noise, noisy. (3.628)

gan $n$. song. $<\mathrm{B}(8.83)$

gan-a vt. wear, put on of clothes, esp. those worn below the waist: underpants, pants, lunggi, loincloth leng-ti, but also sari; put on or wear jewelry of all sorts, necklace, earrings, bracelet, watch, etc.; wear, dress oneself, in contrast with pat-a dress someone else. (6.53)

gan-a chin-a $n$. clothing, general term. (6.521)

gan-chi $n$. bed of firewood for cremation. (5.423)

gan-da $p p$. 1. as for, concerning, by contrast, instead, on the other hand, (follows nominative): ang-a gan-da i-ang-na man--ja as for me, I cannot go. 2. because of, therefore, on account of, (follows dative -na or occasionally genitive -ni): ang-na gan-da because of me. (1.13)

gan-dap-a $v t$. wear on top, on the outside. (6.53)

gan-di $n$. a small, smelly beetle. (4.469)

gan-di mo-sol-a $n$. a dill-like crop with tiny whitish spray flowers, which is grown and eaten. (5.23)

gan-di-go-ja-ri $n$. a tree with large fibrous leaves, bunches of soft purple grape-like fruit; not the same and not as valuable as the go-ja-ri. $<$ B (4.632)

gan-dik-a vi. uncomfortable, not good for wearing. (6.53)

gan-ding-chin-ding $n$. clothes, garments, lower and upper garments. $(6.521)$

gan-do, gan-du $n$. old-fashioned man's loincloth, (same as reng-ti).

$(6.527)$

gan-du-ri $n$. navel, belly button. (6.245)

gan-sit-a vt. change clothes, esp. after a bath. (6.53)

gan-ti $n$. a small fly that makes a noise but does not bite. (4.462)

gan-to-a vi. comfortable, good for wearing. (6.53) 
gan-tong $n$. stick, cut stalk of a plant such as manioc, stick of firewood.

gan-chat-a vi. dense, thick, crowded, of clouds, fog, forest, people.

gan-dil-eng $n$. knife made from bamboo. (5.832)

gan-drak $n$. a white jumping frog-like reptile, about $20 \mathrm{~cm}$. long that lives in trees in the forest, and makes a ga-ak sound in the rainy season. (4.447)

gan-sang $n$. platform for an old style bamboo house, platform for sleeping made from boards or bamboo. (5.637)

gan•-sang nok nph. bamboo house built on gan•-sang type platform. (5.631)

gang $n$. river, smaller than a no-di. $<\mathrm{B}(4.252)$

gang-a vi. sexually excited, aroused. (6.72)

gang-bik-a vi. bend somewhat but less than gong-jol-a. (2.318)

gang-chu $n$. outer (distal) part of a bird's wing. (4.471)

gang-ga $n$. a type of at-te (bamboo knife) that has a long curved blade. $(5.832)$

gang-get-a, gang-git-a vi. bend one's back to the front or side; bent over, as an older person; bent up, of a dog's tail. (9.348)

gang-gi-sim $a d v$. blackish, any dark color; or any cool color, even if light. $(3.534)$

gang-gil-a vi. fall over, as crops in the wind; fall down, lie down, esp. fall on one's back. (9.212)

gang-gip-bok $a d v$. somewhat white, off white, light colored, gray, even darkish gray. (3.531)

gang-git-a vi. pointed up, like a dog's tail. (2.317)

gang-git-chak $a d v$. somewhat red, reddish, dark red. (3.532)

gang-gu-a $n$. mosquito. (4.462)

gang-ma $n$. blackhead of the skin. (8.955)

gang-ma $n$. white pus that can be squeezed from pimples. (6.29)

gang-pak $n$. the part of a wing that is close to the body. (4.471)

gang-pang-a vi. on one's back, sleep on one's back. (9.343)

gang-ping $n$. upper part of a wing, part near the body. (4.471)

gang-rang-a vi. fall, on the back or side. (9.326)

gang-rim-it $a d v$. somewhat yellow, yellowish; occasionally blue or green. $(3.532)$

gap-a vi. full, of any container. (2.26)

gap-et-a $v t$. fill, cause to be full. (2.26)

gap-ket-a vi. filled up tight, no more space, squeezed in, as in a bus, filled with clouds: gim-bi chi-cha gap-ket-a the water pot is filled full with water. (2.26) 
gar-jen $n$. guardian, person responsible for someone else, as a parent. $<\mathrm{E}$ $(8.41)$

gat $n$. guard, forest guard. $<\mathrm{E}(8.41)$

gat-a $v t$. eat holes in paper or cloth, of insects. (4.487)

gat-a vt. give a name, confer a name at baptism. (7.18)

gat-a vt. place on, load, put on top of; cover, as one's head; put or carry on the shoulder or head; pile up, as cloth; build a mud house; set trap; hang clothes, jawbones; attach; give a second or replacement spouse; put into a cradle; give a name to someone, as at baptism: ja·-chok gat-a sit with ankles or lower legs crossed: man-di pi-sa jo-loi gat-e mo-jim-a the baby having been put in the cloth cradle, rocks. -gat- aa. add, cover, put on top: so•-gat-a push into fire; de•-gat-a lift, load; ra·-gat-a adopt a child. (9.62)

gat-dap-a $v t$. put on top of; pile up, as dirt. (9.647)

gat-krang-a vt. build one layer on top of the one below, as the layers of crossing pieces supporting a floor. (9.647)

gat-su-a $v t$. place on first, as set out food before people come. (9.68)

gau-a vi. come off, crack open, of wood when chopped, of rice husks in a rice pounder; easily separated, flake off, break apart readily, of skin, bark; open up, of plant husks; not tough. (9.282)

ge-gak, ge-gat $n$. late stage of a maturing insect that is able to fly. (4.474)

ge-na-si, gi-na-si $n$. a green bean with a broad pod that is eaten along with the seeds. (5.23)

ge-na-si bi-gil $n$. edible pod of ge-na-si beans. (4.676)

ge•-, gi-- cls. general classifier, residual category classifier for objects that lack any other clear classifier, esp. for small man-made objects, pens, cups, houses, etc., (A·chik). (1.31)

ge-a, gi-a vt. plant into a hole, plant seeds individually or a few at a time, for maize, trees, etc., plant rice seedlings; contrasts with go-a sew, broadcast. (5.14)

ge'-bot-a, gi-bot-a vt. plant between other plants so as to fill in gaps. $(5.14)$

ge•-eng-a vi. come untied, be untied, of string, lunggi. (9.65)

ge-jrot-a, gi-jrot-a, gi-jot-a vt. plant between other plants, so as to fill in gaps, as to replace rice seedlings that have died. (5.14)

-gek-, -gek-gek- $a a$. forcefully, in quantity: ru-gek-a pour in large quantity, filter ashes to get soda; ding•-gek-a very hot; mal-gek-a very tame, of animals; ka-ding-gek-gek-a laugh loudly, constantly; jom-gek-geka whimper, groan when sick; du-gek-gek-a feed by force. (7.56)

gek-a vi. taste too strongly of salt, soda, or chilies. (3.684)

gek-a, ru-gek-a vt. add to curry, of spices, soda, condiments. (5.46) 
gek-gak-a vi. talk a lot, chatter; disturb, make much noise, of animals. gek-gak-gek-gak $a d v$.: a-chak gek-gak-gek-gak dak-e sing-ing-a the dog is barking noisily. (7.15)

gek-gek $a d v$. very hot, of the sun, of water: gek-gek dak-e chi ding·-et heat the water very hot. (3.673)

gek-gek-a, jom-gek-gek-a vi. groan when sick, whimper. (7.29)

gel-a $v t$. avoid, go a long way round so as not to meet. (9.317)

gel-grik-a vi. avoid each other, pass each other on the road going in opposite directions. (9.317)

-gen pvs. future, $(\mathrm{A} \cdot$ chik equivalent of Mandi -no-a). (0.211)

gen-chi $n$. round winnowing tray. (5.852)

gen-da $n$. infant, baby who is not yet walking. $<\mathrm{B}(6.822)$

gen-da-pul $n$. marigolds, small yellow flowers. (5.28)

gen-ji $n$. tee-shirt, undershirt, knit shirt. $<\mathrm{B}(6.521)$

gen-gen-a vt. make a waving pattern on the bamboo for an altar by scraping off the green color. (8.58)

geng-a $v t$. echo in gil-a geng-a beg. (8.449)

geng-gok-a $a d v$. curled up, sleep with knees drawn up and face down. (9.343)

geng-kon-a vi. curl up and turn, as a dog turns when lying down. (4.482) geng-reng $n$. a filter for soda. (5.438)

geng- cls. classifier for rice seedlings, blades of grass, thatch. (1.38)

geng-gang-bak-gang $a d v$. disorderly, as branches piled around. (2.317)

gep-a $v t$. tie with two parallel pieces holding something between them, esp. for the encircling pieces of a basket top that hold the tops of the vertical side pieces. -gep- aa. tie together: ka-gep-a tie together; cho-gep-a tie up in a folded banana leaf bundle. (5.856)

gi-ar $n$. gear of a bicycle. $<\mathrm{E}(5.872)$

-gi-ja pvs. the one who is not, those who are not; negative of the -a nominalizer; words suffixed with -gi-ja can be used either as a noun or to modify a noun: dal-gi-ja nok the house that is not big; dal-gi-ja-ko nik-jok (I) saw the one that is not big. (0.218)

-gi-ja-gip-a pvs. negative of the -gip-a nominalization, allowing words to which it is suffixed to be used as a noun or to modify a noun; the one who is not, those who are not: kat-ang-gi-ja-gip-a man-de the man who does not run away. (0.218)

gi-jem-a $v t$. echo in git-ak-a gi-jem-a cut into pieces. (9.573)

gi-jip $n$. fan. (5.835)

gi-jo-a, gi-ju-a vi. make a disturbance, fuss, screech, make noise, of children, of flocks of birds. (7.29)

gi-la, gi-la-bi-du $n$. a large woody vine with a fruit, said to be poisonous enough to be deadly if eaten. $<$ B (4.655) 
gi-na-si, ge-na-si $n$. a green bean with a broad pod that is eaten along with the seeds. (5.23)

gi-ro, gi-ru $n$. joint of the body, protruding bones such as ankle bones, knee; node of bamboo; bump, branch that sticks out from a node of bamboo. $<$ B $(6.28)$

gi-sep-o $p p$. between, among, (follows genitive -ni). (1.11)

-gi-si dns. old and dried out, of plants, wood, tree. (3.74)

gi-si-a vi. echo in ni-ri-a gi-si-a quarrel, get along badly (8.32)

gi-sik $n$. the mind. (7.41)

gi-sik cham-a vph. think about, think over. (7.41)

gi-sik chan-chi-pil-a $v p h$. change one's mind. (7.41)

gi-sik chon-a vph. not know much; narrow minded, (lit. mind-small). (7.41)

gi-sik ja-brang-a $v p h$. worry. (7.764)

gi-sik jom-a, gi-sik-o jom-a $v p h$. upset, unhappy, (lit. sick in the mind). (7.742)

gi-sik ken-chak-a $v p h$. suspect. (7.42)

gi-sik mik-ta $v p h$. good mind, good character, knowing, knowledgeable.

gi-sik nang-a $v p h$. be interested in, concerned with, attentive. (7.47)

gi-sik ong-chak-a vph. sympathize, feel grief for. (7.741)

gi-sik ra-a, gi-sik ra'-rik-a $v p h$. remember. (7.45)

gi-sik ra'-pil-a vph. change one's mind, (lit. take back the mind). (7.41)

gi-sik rak-a vph. intelligent, (lit. mind strong). (7.44)

gi-sik ron-a vph. pay attention, give one's mind to something, (lit. give the mind). (7.47)

gi-sik sa-a, gi-sik-o sa-a $v p h$. be upset, unhappy, (lit. sick in the mind). (7.742)

gi-sik sim-sak-a $v p h$. suspect. (7.42)

gi-sik sok-a $v p h$. smart, (lit. the mind reaches); gi-sik sok-ja not smart. (7.44)

gi-sik srang-a vph. without worries, unable to tell a lie, clear of mind. (7.764)

gi-sik su-ang-su-ang $v p h$. without hope, frustrated. (7.764)

gi-sik ting--ting-a, gi-sik ting-ting-tang-tang $v p h$. anxious, worried. (7.764)

gi-sik tom•-tom-a vph. calm, peaceful, without worries, (lit. peaceful mind). (7.764)

gi-sik-a vi. crow, cock's crow. (4.476)

gi-sik-pil-a vi. reform, turn over a new leaf, (lit. reverse the mind). (7.41)

gi-sil-a vi. separate from parents in eating and economic arrangements but not necessarily change residence, of a young married couple. (6.657) 
gi-sim, gi-sim-a, sim-a vi. black; by extension: any cool color, even blue or green; any dark color. (3.534)

gi-sim-mrang-mrang $a d v$. blackish, blue, green. (3.534)

gi-sim-pru-pru adv. very black, pure black. (3.534)

gi-a, ge-a vt. plant into a hole, plant seeds one or a few at a time, as for maize, trees, etc., plant rice seedlings, (see ge•- for compounds). (5.14)

gi-gang-gil-a, ki-me gi·-gang-gil-a vt. raise up tail, as a cow does when eliminating. (4.488)

giil-ja-nok $n$. church building. $<\mathrm{B}(5.624)$

giit $n$. song, $(\mathrm{A} \cdot$ chik $)$. (8.83)

gik dak-e $a d v$. suddenly. (2.728)

gil- cls. classifier for crosswise slices, as loops of pineapple, etc. (1.37)

gil-a $v t$. beg for alms, collect money in church. (8.449)

gil-as $n$. drinking glass. $<\mathrm{E}(5.434)$

gil-chon-a vi. small, of flattish things, fried cakes, boards, mats, but not for things as thin as those classified by king-. gil-chon-gil-chon adv. (2.211)

gil-chrak-a vt. peel away skin, as from a burn or a wound. (8.953)

gil-dal-a vi. big, of flattish things. (2.211)

-gil-gil- $a a$. on the surface: kam-gil-gil-a burn on the surface; wi-gilgil-a plow shallowly. (2.319)

gil-gip-a vi. enough to cover. (3.341)

gil-gok-a vi. peel off, of bark; lose skin, shed skin, peel, as from a burn or injury. (8.953)

gil-i-geng-i cha-gip-a $n$. beggars, (lit. those who eat by begging). (8.41) gil-kri-a vi. clear up, of clouds, dirty water. (4.323)

gil-ma-gil-ma $a d v$. big, of flattish things, in large flakes, as for peeling bark; used for more than one thing. (2.211)

gil-min-ik-a vi. improve, flatten, of skin after a disease. (8.953)

gil-prak-a, gil-ru-ru-a, gil-prak-gil-ru-ru-a vi. peeling and oozing, as from a burn. (8.953)

gil-si-ri-a, gil-sri-a vt. shed, as a snake sheds its skin; peel, peel off bark; peel off loose skin, as from a skin disease: chip-pu bi-gil gil-si-ri-ing-a the snake is changing its skin. (4.482)

gil-sin-ik-a vi. be slightly cut, slightly wounded skin. (8.955)

gil-we, gil-wi $n$. very shy monkey-sized animal that lives in trees. (4.419) gil-e-o-gil•-e-o $a d v$. glowing, of coals in a small fire. (5.421)

gil·-jik-gil•-jik adv. flaming up strongly, putting out heat; rich and having a lot of possessions. (5.425)

gim-a, wa gim-a vi. feeling of the teeth after eating sour things. (3.682)

gim-a-a vi. be lost, of things. (9.538)

gim-a-et-a $v t$. lose, cause to be lost. (9.538) 
gim-bi $n$. large water pot, bigger than a dik-te. (5.432)

gim-bi rong-dal-a $n$. large gimbi. (5.432)

gim-bil $n$. tree with oval reddish $20 \mathrm{~cm}$. leaves that are used to wrap biris.

This is the tree to which one ties his cow on the trip to Chik-mang after death, (Beng. kum-bir). (4.632)

gim-ik $n$. all, every. (3.343)

gim-ik jai-ga-o $l w$. in every place, everywhere. (2.13)

gim-in $p p$. because of, due to, (follows genitive -ni): neng-be-a-ni gimin because of being very tired. (1.13)

gin-a $v t$. echo in dok-a gin-a create, by god. (8.52)

gin-ang $p p$. with, including, along with, (follows nominative): ang-a gin-ang along with me. (1.16)

gin-di n. dry powder, such as milk powder, flour. (5.405)

gin-dil-gin-dil $a d v$. grinding up into dust, of dirt, earth. (9.649)

Gin-gip-a $n$. a name for the Christian God, usually an echo of Dok-gip-a the forger: Dok-gip-a Gin-gip-a. (8.54)

-gin-i, gin-ing num. two. gin-i-gip-a $n$. second. (3.3702)

-gin-ok pvs. immediate or intentional future, $(\mathrm{A} \cdot$ chik equivalent of Mandi -na-jok). (0.211)

gin-chi-ra $n$. sieve, tool for sifting. (5.852)

ging $n$. nose, snot. (6.22)

ging et-a vi. blow nose. (9.44)

ging-bi-bal $n$. nose ornament, (lit. nose flower). (6.562)

ging-brak, ging-brak-brak adv. flat-nosed. (6.42)

ging-brek, ging-brek-brek, ging-ki-brek adv. having a runny nose, having a face covered with snot. (6.58)

ging-brong, ging-brong-brong dak-a $a d v$. having a large nose or large nostrils. (6.42)

ging-chep-ta $a d v$. flat-nosed, characteristic nose shape of Mandis. (6.42)

ging-chi-deng-deng $a d v$. long-nosed. (6.42)

ging-chi-mik-chi ong-kat-ing-a $v p h$. with running nose and eyes, as from smoke or a cold. (8.93)

ging-dep, ging-chi-dep-dep $a d v$. flat-nosed, short-nosed. ging-chidep-a vi. (6.42)

ging-do-reng, ging-do-reng-reng $a d v$. having a long nose, hawk nose. (6.42)

ging-gre, ging-gri $n$. pus, nasal mucus, phlegm. (6.29)

ging-ki $n$. nose dirt, snot, dried snot, (lit. nose-dung). (6.29)

ging-kol, ging-kil-ok, ging a'-kil-ok $n$. nostril, (lit. nose hole). (6.22)

ging-kol-ni kim-il $n$. nostril hair. (6.25)

ging-na-bak $n$. central septum of the nose. (6.22)

ging-rik-ing $n$. lower edge of the nose and nostrils. (6.22) 
ging-ro-a, ging-ru-a vi. long-nosed, pointed-nosed. (6.42)

ging-sep $n$. outer edge of the nostril, crease on the side of the nostril. $(6.22)$

ging-si $n$. blood that comes from the nose. (6.22)

ging-si-mrang, ging-si-mrang-mrang, ging-si-mrang-ging-si-mrang $a d v$. good smelling, fragrant, good smell enters the nose. (3.688)

ging-sik-a $v t$. give a strong feeling in the nose from the smell of chilies or smoke or just before sneezing, (lit. penetrate the nose). ging-sik-gingsik $a d v$. (3.688)

ging-sot-ti $n$. small blemish, pimple on the nose. (8.955)

ging-ti, ging-to-ti $n$. tip of nose. (6.22)

ging-ting $n$. nose, tip of the nose. (6.22)

ging-tong $n$. the snout of an animal, esp. a pig's snout. (4.473)

ging•-gen-a vt. make a decorative zigzag pattern on bamboo, as for an altar. (5.856)

-gip-a dns. suffix for kinship terms when used for reference rather than as terms of address: Nel-son-ni ma•-gip-a Nelson's mother. (6.64)

-gip-a pvs. nominalizing suffix, the one who, those who: pi--sa-ko chikgip-a a-chak the child-biting dog, the dog that bites the child. (0.218)

gip-a vi. big enough or long enough; sufficient to fully cover, reach, of cloth, thatch, etc. (3.341)

gip-ak- cls. classifier for loads, as firewood. (1.34)

gip-ak-a vt. embrace, hug, by people or things; carry child in arms or on hip without a carrying cloth; take on the breast, take on the knee. (9.531)

gip-ak-chet-a vt. embrace tightly, closely, wrap arms around, grab and hold something or someone, as a child, a lover, a victim. (9.531)

gip-ak-grik-a vt. mutually embrace. (9.531)

gip-bok, gip-bok-a, bok-a vi. white, light colored. (3.531)

gip-bok gi-sim dak-a $a d v$. in black and white, not colored. (3.539)

gip-bok-rong-rong $a d v$. pure white. (3.531)

gip-eng $n$. width, one of the three spatial dimensions. (2.34)

gip-eng-pang $n$. turned sideways, crosswise; perpendicular; not saying things straight. (2.34)

gip-eng-pang bil-a $v t$. roll up starting from short dimension. (9.645)

-gip-i dns. oldest sibling, oldest brother or oldest sister. (6.64)

gip-ik-a vi. come out by the roots; fall over so that roots come out, as a tree in the wind. (9.212)

gip-in $n$. other, another: -gip-in num. another, different: sak-gip-in someone else; mang-gip-in another animal. gip-in-gip-in $n$. others, various. (3.437)

gip-ok $n$. egg white. (4.475)

gip-u-a vi. leak through, pass through, as through a sieve; ooze, of a wound. (2.22) 
gip-u-rong-rong-a vi. fit through something small, such as a sieve. (2.22) -git-a cm. like, in that way, resembling: man-di-git-a like a person. $(0.12)$

git-ak-a vt. cut into small pieces, of things such as meat or tobacco. git-ak-pri-pri $a d v$. cut into very small pieces. (9.573)

git-al vi. new. (3.74)

git-chak, git-chak-a vi. red; by extension: reddish brown, orange. (3.532)

git-chak-gip-bok adv. pinkish, salmon colored, (lit. red-white). (3.532)

git-chak-mrang-a vi. reddish, such as the color of middle-sized tiger (mat-cha) that is neither striped nor spotted; color of the sky at sunset. git-chak-mrang-mrang $a d v$. (3.532)

git-chak-rim-it $a d v$. orange, (lit. red-yellow). (3.532)

git-chak-rong-rong $a d v$. bright red. (3.532)

git-chak-sri-sri $a d v$. very red, bright red. (3.532)

git-cham, git-cham-a vi. old, of things. (3.74)

git-chem $n$. rice beer that is not the first straining to be taken from the pot. (5.403)

git-chi $n$. a small, narrow-bladed hoe, for weeding and digging up roots. (5.831)

-git-chi- $a a$. in an insulting way: ka-ding-git-chi-a laugh insultingly; in-git-chi-a insult by saying something. (7.27)

git-chil-a vi. slip and fall down, of a person, esp. fall on one's face. (9.326)

git-ching-a $n$. bent over but less than gang-jol, of a tree, wall, etc.; tipped, of a cooking pot; a bit past noon when sun bends down past its high point. (2.318)

git-chit-a vi. torn, torn off, of cloth; broken up, of a fence. (9.292)

git-chit-drak-a vi. badly torn, with large tears, of cloth, baskets. (9.292)

git-chit-pri-pri-a, git-chik-pru-pru-a vi. with lots of tears, but not torn into pieces. git-chit-pru-pru $a d v$. (9.292)

git-chit-tong-a vi. torn apart. (9.292)

git-chit-tring-trang-a vi. torn in many places but not into pieces, tattered. (9.292)

git-dap-a vi. in layers, one on top of the other, piled up. git-dap-git-dap $a d v .(9.647)$

git-i $n$. younger siblings. (6.64)

-git-ik- $a a$. suddenly, unexpectedly: si-git-ik-a die suddenly; sok-gitik-a-ri-jok just arrived unexpectedly; wal-o-ni dip-at jom-git-ik-a, i-ba-na man-ja-jok since the nighttime he has been unexpectedly sick and couldn't come. git-ik-git-ak $a d v$. suddenly, unexpectedly. (2.728)

git-ik-a vi. move, squirm, wiggle, of people or animals; be shaken, as in an airplane. (4.482) 
git-ik-git-ak adv. suddenly, unexpectedly. (2.728)

git-il-o $l w$. a bit past noon. (2.52)

git-ok $n$. neck, throat; narrow place, neck of a pot. dns. narrow place: jak-git-ok wrist; ja·-git-ok ankle; chi-git-ok narrow place in a stream. (6.241)

git-ok-do•-chi $n$. adam's apple, (lit. neck-egg). (6.241)

git-ok-nal $n$. gullet, esophagus, (lit. neck pipe). (6.27)

git-ok-reng $n$. back of the neck. (6.241)

git-ok-srang-ja vi. speak quietly, unclearly, hoarse. (7.15)

-git-tam num. three. git-tam-gip-a n. third. (3.3703)

git-tam-a vi. urinate. $n$. urine. (9.49)

git-tam-bu-a vi. pee in pants, wet one's bed. (9.49)

git-tam-chi-rot-a vi. pee just a little. (9.49)

git-tam-chip-a vi. pee in pants, wet one's bed. (9.49)

git-tam-prak-a vi. pee a lot. (9.49)

git-tang-a, git-tang vi. living, not dead. (4.690)

git-tang-gi-si $n$. exchange labor, the first day is git-tang (living), the day the labor is returned is gi-si (dead). (8.433)

git-tang-si-si, git-tang-sik-sik $a d v$. not yet dry, of firewood. (5.423)

git-tim $n$. section of a village comprising several houses or clusters of houses, neighborhood. (8.22)

git-ting vi. green, unripe, raw, uncooked. (4.692)

git-u-a vi. boil noisily, bubble. (5.46)

git-u-ra $n$. a water lizard that is a bit smaller than an a-ring-ga. (4.441)

git-u-sru-a vi. boil over. (5.46)

go-a, gu-a vt. throw, scatter; broadcast seeds, plant; cast fishnet; play out cards; shoot gun. (9.54)

go-al-i-nok, gu-al-i-nok $n$. cattle shed, cattle house. $<\mathrm{B}(5.622)$

go-ba-rip-rip-a vt. scatter, throw around. (9.54)

go-brap-a, gu-brap-a vt. sew excessively, broadcast excessively, in a less controlled manner than go-a. (5.14)

go-chrak-a vt. throw or broadcast vigorously, forcefully, as rice grains, so that they fly all over the place; even less controlled than go-brap-a. (5.14)

Go-e-ra $n$. name of a mit-e (spirit) that makes thunder. (8.54)

go-et-a vt. throw. (9.54)

go-gak-a $v t$. throw at: rong-te-cha do-ko go-gak-a throw at the bird with a rock. (9.54)

go-ja-ri $n$. sal tree, (shorea robusta). <B (4.632)

go-jron-a, gu-jron-a vi. fall down flat, from sickness or pain. (9.326)

go-kring-krang-a vt. throw around, throw in an uncontrolled way, throw roughly, in various directions or off to the side. (9.54) 
go-min-da, gu-min-da $n$. a round squash that lies on ground when growing. (5.23)

go-pret-a, gu-pret-a vt. strike, of lightning; throw hard and break. $(4.344)$

go-pri-pra-a $v t$. scatter, throw around but more gently than go-sri-sraa. $(9.54)$

-go-ra dns. thicket: wa-go-ra bamboo thicket; bring-go-ra thick forest. (4.630)

go-ra, gu-ra $n$. horse. $<\mathrm{B}(5.35)$

go-rai-a, gu-rai-a vt. turn, turn around, as the knob of a hurricane lamp; stir tea, etc. $<$ B $(9.645)$

go-re ro-a, gu-ri ru-a vi. do a circle dance, in which women stand in the center and men circle around them. (8.83)

go-re-a, gu-ri-a vi. turn around, revolve, like clock hands; turning, curving, of a road; wander about, stroll. $<\mathrm{B}(9.214)$

go-re-go-re, gu-ri-gu-ri, gu-ri-e-gu-ri-e $a d v$. wandering about; revolving. $<\mathrm{B}(9.327)$

go-re-rik-rik-a, gu-ri-rik-rik-a vi. turn again and again; follow someone around repeatedly. (9.327)

go-ri $n$. watch, clock. $<\mathrm{B}(5.838)$

go-rim-rim-a $v t$. roll along the ground. (9.54)

go-ring-rang-a vt. throw back and forth, swing back and forth. (9.54)

go-ring-ri-a vt. swing something in a circle, as on the end of a string.

go-ru, gu-ru $n$. a plant with edible leaves, (same as men-da). (5.23)

go-sreng-a $v t$. pass to someone by rolling or sliding along the ground. $(9.54)$

go-sri-sra-a $v t$. throw things around, this way and that, as small children do with rice when eating, scatter. (9.54)

go-stap-a $v t$. throw forcefully to the ground. (9.54)

go-tat-a $v t$. throw at forcefully, with stones, sticks, etc., as at people, dogs, pigs: rong-te-cha a-chak-ko go-tat-a throw a stone at the dog. $(9.54)$

go-wek-a vt. throw mud against a wall to build it up and solidify it during house construction. (5.631)

go-long $n$. vine with huge bean-like pods and red seeds. (4.655)

go-ok-a vi. come apart, come loose, come out of, as a tool handle, cork; fall out, be pulled out, plucked, of hair, of feathers; fall down, of a lunggi; -gok- $a a$. separated, broken apart. (9.572)

-gok- $a a$. bent over: deng-gok-a bow deeply; geng-gok-a sleep with knees drawn up; bam-gok-a bow head or body, bent over, of people. $(9.348)$ 
-gok-, -go-ok- $a a$. broken apart: separated: dok-gok-a hit and separate, as to knock off a handle; rong-gok-a break off a round thing, such as a glass bottom; gil-gok-a cracked and peeling, of skin. (9.282)

gok-a vi. want to, be inclined, enthusiastic. (7.725)

gok-a-rek $n$. small tree, the size of sugar cane, that grows beside river with big flowers. (4.632)

gok-chip-a, na-chil gok-chip-a vi. disturbed in the ears, tired of too much noise, don't want to hear: bol-a-ko kin-a-e na-chil gok-chip-a hearing the noise, the ears are disturbed. (3.628)

gok-gip-a $n$. hard working person, eager person. (7.725)

gok-gok-a vi. with head down, bend over, bend head or bend from waist, bend of trees. (9.41)

gol-a $n$. a bundle of thatch. $<\mathrm{B}(5.632)$

gol-mo-ris $n$. pepper. $<\mathrm{B}(5.405)$

gol-po $n$. story. $<\mathrm{B}(7.12)$

gol•-, gol, go•-ol $n$. stick. gol-- $c p$. categorizing prefix for sticks: gol-chok arrow; gol·-a-reng long pole. (4.672)

gol-a-rang $a d v$. tall and thin, of people. (6.44)

gol-a-reng $n$. a long pole, long stick, such as might be used for knocking down fruit. (5.833)

gol-chok $n$. arrow, tip of an arrow; stake, pointed stick such as is used to insert in a house wall. (5.845)

gol-dak-a vi. straight, stick like, sticking up straight, as an uncurled dog's tail; lazy, never bending to work. (2.318)

gol-dang-dang $a d v$. tall, like a stick, of a long pole, rude when referring to people. (6.44)

gol·-dik $n$. small stick, walking stick or cattle prod, shorter than a gol•-areng. (5.833)

gol-ding-ding $n$. chips of wood. (4.673)

gol-gol-a vi. flow, fall, of water, as when poured from a pot or when a pot leaks. (9.273)

gol-ka-tek $n$. a small stick used to beat gongs. (8.85)

gol-ka-ti $n$. a small stick used to beat a drum or gong. (8.85)

gol·-ki, gol·-ki-ki, gol·-ki-rek-rek $n$. something neglected, shabby, lean and thin, esp. a dog, but even a person. (9.292)

gol·-ki-dang-dang, gol·-ki-ki $a d v$. thin, stick-like. (2.312)

gol-krang-krang dak-e cha-deng-a vi. stand up straight. (9.346)

gol-kreng-a vi. tall and skinny, thin, not fat, of a person. gol-krengkreng $a d v .(6.46)$

gol·-si-ri $n$. chips, as from wood or bamboo. (4.673)

gol-tak-tak-e cha-deng-a vi. stand up straight. (9.346)

gol-teng $n$. joking term for penis, (lit. stick). (6.249) 
gol-teng-teng $a d v$. very poor, without clothes, implies having so few clothes that the gol--teng (stick, i.e. penis) shows. (8.441)

gom $n$. wheat. $<\mathrm{B}(5.23)$

gon-do-ras $n$. gardenia. $<\mathrm{B}(5.28)$

gon-ta, ghon-ta $n$. bell, gong. gon-ta- cls. classifier for hours. $<\mathrm{B}$ $(2.53)$

gon-gon-a vi. come persistently to someone to get something, as a child to a parent; as a young man or young woman comes persistently to the other; pester. (7.723)

gon-gon-a $v t$. stir food when cooking, as rice, frying food. (5.46)

gong $n$. a reed with stalks of finger thickness, almost lily like leaves, and as tall as a person. Central part can be eaten. (4.652)

gong-brap-a vi. fall over, as heavy rice plants in the rain or wind. (9.212) gong-gang $n$. a large type of stinging black ant. (4.465)

gong-ge bak-e bol-a vph. talk indirectly, in a curved way, not sticking to the subject. (7.13)

gong-ge-a, gong-gi-a vi. bent, as wire or cord; curved, winding, of a road; zigzag; less tightly bent than gong-jol-a but more than gang-bika curving back and forth. $a d v$. gong-ge-gong-dang. (2.318)

gong-ge-a, gong-gi-a, gong-ge-git-ik-a vi. disobey, disagree, not accept another's word, (lit. not straight, crooked). (8.33)

gong-ge-a-o $l w$. around the bend, out of sight. (3.54)

gong-ge-bak-a vi. curve back and forth, waving, zigzag, of roads, lines of trees, when walking). (2.318)

gong-ge-gong-got, gong-gi-gong-got, gong-ge-gong-grot $a d v$. winding back and forth, of a road, a line of trees, when running, writing; twisted, of a bamboo piece; zigzag. (2.318)

gong-grot-grot, gong-got-got $a d v$. bent over, curled, as bending rice plants, a bird's tail. (2.317)

gong-jol-a vi. bent way over, as in the wind, of trees, bamboo, rice plants; bent more tightly than gong-ge-a, but not fallen all the way to the ground. (2.318)

gong-ting-a vi. bend to the front at the waist. (9.348)

gong-ting-grik-e chu-a vph. sleep back to back. (9.343)

gong-a vi. be inclined, want to do something. (7.725)

gong-gak-a vi. broken apart, fall off, of tool handles, of posts after being eaten by ants, of fruit or branches from a tree. (9.282)

gong-rak-a vi. fast, rapidly, as when walking, running. (2.726)

gong•-rak-e $a d v$. echo in jak-rak-e gong•-rak-e quickly, fast. (0.5)

gong-rak-sak-sak $a d v$. quickly, while running quickly. (2.726)

gong-sa num. two takas, a two taka note; an idiosyncratic and idiomatic meaning where 'one taka' would seem to be a more reasonable translation, (cf. gong-bing-a five takas). (1.38) 
-gop- $a a$. bent over: chu-gop-a sleep with legs bent up; bi-gop-a fallen over, of a tree; bam-gop-a bow deeply, bend over, of people. (9.348)

gop-a $v t$. cook by wrapping food in banana leaves. (5.453)

gop-ang, a•-gop $n$. a large hole in a tree or in the ground, larger than an ha-kil-ok. (2.38)

gop-ma-a vi. very fat, chubby, of people, animals. gop-ma, gop-mama, gop-ma-ru-ru $a d v$. (6.48)

gop-ram $n$. grave, burying place. (6.79)

gra-a vi. not sticky, of dry flour, dirt, rice, etc. (3.668)

gra-gra $a d v$. light shower: mik-ka gra-gra wa-ing-a it is raining lightly. $(4.325)$

grak-grak, grok-grok $a d v$. in gulps, of drinking. (5.474)

gran $n$. dried, dried in the sun, of meat or fish: wak-gran dried pork; ma`-su-gran dried beef; kut-chi-a-gran dried eel. (5.401)

grang $n$. wing, feather. (4.471)

grang-a vi. having open spaces between the pieces, of baskets, etc. grang- $a a$. widely spaced: ap-grang-a widely spaced of basket pieces, made with open spaces; wa-grang-a nicked, not continuously, of a knife blade; ka-grang-a tied together with open spaces, of a fence. granggrang $a d v .(5.856)$

grang-jip-a vi. flap wings slowly, (lit. wing-fan). (4.486)

grap-a vi. cry, weep, wail for the dead. (7.29)

greng $n$. bone. (6.28)

grep-grep $a d v$. hurting as well as itching, of an insect bite. (3.64)

gri $p p$. without, (follows nominative): ang-a gri without me. (1.16)

gri, gri-gip-a, gri-tang, ang-gri $n$. cross nephew, woman's brother's son, man's sister's son. (6.617)

gri-a, jom-a gri-a vi. get better after being sick, improve. (8.91)

gri-tong $n$. a wild cat that is bigger than a mat-cha-a-rek. (4.416)

-grik- aa. mutually, each other: a-gan-grik-a converse, talk to each other; sin-grik-a like each other; grong-grik-a meet each other. (0.41) grik-a vi. clear, not muddy or cloudy, of liquids. (3.714)

grik-a vt. do a war dance with headhunting sword and shield, in which one shouts out while dancing. (8.83)

grik-jang-jang $a d v$. clean, clear, of water. (3.714)

grim $p p$. together, close together, in a group, thick with, (follows -ming): ang-ming grim together with me. -grim- aa. together: pin-grim-a thatch together, in a group of people; nam-grim-a get along, be friendly; in-grim-a talk at the same time. (1.16)

grim-grim $a d v$. together, along with: ang-ming grim-grim kat-angjok ran away along with me. (2.11)

grin-a vi. slide down, collapse, of earth. (9.212) 
gring, do-gring, du•-gring $n$. a small basket in which domestic fowl can nest, or in which they can be carried to market. (5.852)

gring-a vi. howl, of dogs, jackals. (4.476)

gring-gring-grang-grang $a d v$. woven with spaces between the pieces, of baskets, etc. (5.856)

grip-a vt. cover, pot, basket, close up, shut in: da-kon-cha mi grip-a cover rice with a lid; grip-e don-a enclose, as in a cage. -grip- $a a$. cover: on'-grip-a cover, of clouds; ku·-grip-a cover, of a pot. (9.62)

grip-dap-a $v t$. cover over, as with a plate. (9.647)

grip-et-a $v t$. cover, as rice pot. (9.62)

grip-grap $a d v$. unexpectedly, quickly: grip-grap an-dal-a, du-at sa-ra surprisingly dark without a lamp. (7.48)

grit, grik $n$. sugar cane. (5.23)

gro, gru $n$. length, one of the three spatial dimension. -gro- $a a$. lengthwise, on the long dimension: rat-gro-a cut lengthwise; git-chit-grotear lengthwise. -gro dns. length: bol-gro ridgepole; bi-gro length. gro-gro, gru-gru $a d v$. lengthwise: gro-gro bil-a $v t$. roll up starting from the long dimension. (2.34)

gro, gru vi. legal case, an offense that leads to a legal case. gro nang-a $v p h$. commit an offence, such as theft or adultery, that leads to a legal case. (8.13)

gro cha'-a $v p h$. receive monetary compensation as a result of a legal case.

gro dak-e adv. lengthwise. (2.34)

gro gam-a $v p h$. pay compensation at a legal case. (8.15)

gro nang-e pi-sa man-a $v p h$. have an illegitimate child, (lit. commit an offense, get a child). (6.657)

grok- cls. classifier for amounts drunk at one time. (1.38)

grong $n$. horn of cattle, deer, etc., feelers of an insect. (4.473)

grong-a $v t$. meet. (9.317)

grong-grik-a vi. meet each other. (9.317)

grop-a vi. fall down, collapse, as a house collapses from age; break, of pots, rice plants. -grop- $a a$. fall over, collapse: wil-grop-a fall over, as crops in the wind; bi-grop-a collapse, of walls, houses, trees. (9.212)

-grot- $a a$. scrape, rub: not-grot-a rub up against something in order to scratch oneself; mat-grot-a scrape body against something; ge'-grot-a plant between other plants so as to fill in gaps. (9.554)

gru-a vi. unravel, like knitting. (9.292)

gu-a $n$. areca palm, areca nut. (5.22)

gu-a-mu-ri mo-sol-a $n$. carroty looking plant with an edible fruit. $<\mathrm{B}$ $(5.23)$

gu-a-re $n$. the fence around an altar. (8.58) 
gu-a-si $n$. rib bone; side vein of a leaf. (6.28)

gu-al-a vi. forget. (7.45)

gu-ang $n$. spider. (4.464)

gu-ang-dom-dom-pa $n$. a large kind of spider that lives in the forest and builds large round nests. Said to be able to catch birds. (4.464)

gu-ang-jap-jap $n$. a type of spider that comes into houses. (4.464)

gu-ang-sring $n$. spider web. (4.475)

gu-bol $n$. cow dung. $<\mathrm{B}(4.475)$

gu-gi-rong-chok-a vi. lean way over so butt is highest. (9.348)

gu-gu-a, grap-gu-gu-a, grap-a-gu-gu-a vi. miss, be lonely for, long for; cry in loneliness, wail for the dead. (7.743)

gu-gu-ma-ri, at-te gu-gu-ma-ri $n$. a large type of at-te (bamboo knife) with a hooked shape. (5.832)

gu-me, gu-mi, gu-me-gip-a, gu-mi-gip-a $n$. man or woman's older sister's husband. (6.614)

gu-na $n$. a bulge from a bait (set of wet rice fields), the end of a narrowing bait. <B (5.114)

gu-na, sil gu-na $n$. wire. (5.88)

gu-ni-al, gu-ni-ar $n$. powerful ko-bi-raj (herbal doctor) who can use mon-tros (chants) and medicines to kill people. $<\mathrm{B}(8.51)$

gu-ra, go-ra $n$. horse. $<\mathrm{B}(5.35)$

gu-ri $n$. fog, mist. gu-ri an-dal-a thick fog, dark fog. (4.329)

gu-ri on-a, gu-ri pin-a, gu-ri dak-a, gu-ri ga-ak-a vph. there is fog, it is foggy. (4.329)

gu-ru-ma $n$. locally produced dark sugar, made by boiling the juice of sugar cane. $<\mathrm{B}(5.405)$

Gu-ru-sot-o $n$. the Hindu denomination of the Mandai people and of the Hindu Garos. <B (6.89)

gu-rung-ga-rang $a d v$. roaming around aimlessly. (9.319)

gu-su-a vi. cough, (A•chik). (9.45)

gul $n$. the fence around an altar. (8.58)

gul-a $n$. name of a children's running game. (8.81)

gul-ap $n$. rose. $<\mathrm{B}(5.28)$

gul-gul-a vi. round, spherical; round fried wheat balls. $<$ B $(2.313)$

gul•-gul-a, mik-ka gul-gul-a vi. thunder softly. (4.342)

gup $n$. the end of a bait (group of rice fields). (5.114)

gus $n$. bribe. $<\mathrm{B}(8.442)$

gut-a-ma-ra $n$. a kind of turtle, a kind of chi-jong. (4.445)

gut-am, but-am $n$. button. $<\mathrm{B}(6.598)$

gut-gut kel-ing-a vi. play a game involving hopping on one foot. (9.346)

ha int. word used when giving something to someone, here, please take it, $(0.62)$

-ha, -sa ns. only. (0.14) 
ha re-wa-a, a'-re-wa-a vi. dirty with earth. (3.716)

ha-bi-ja-bi $n$. this and that. $<\mathrm{B}(5.81)$

ha-bu-a, a-bu-a vi. take a bath, bathe. (6.57)

ha-bu-dil-a, a-bu-dil-a vt. bathe, give someone a bath: ang-a pi-sa-ko ha-bu-dil-jok I bathed the child. (6.57)

ha-bu-srok-a, a-bu-srok-a vi. bathe quickly or partially, gently. (6.57)

ha-da-su-rang, ha-du-srang $n$. dragonfly. (4.469)

ha-du-du $n$. children's running game played in a space marked by lines on the ground. $<\mathrm{B}(8.81)$

ha-guk, a-guk $n$. grasshopper. (4.469)

ha-guk-a-reng $n$. a large kind of grasshopper. (4.469)

ha-guk-ging-srok $n$. a kind of grasshopper with a long head. (4.469)

ha-guk-gok-chi-ri, ha-guk-gok-si-ri, a-guk-gok-chi-ri $n$. a small but long kind of grasshopper. (4.469)

ha-guk-ma-du-pa $n$. a big type of grasshopper. (4.469)

ha-guk-mit-im $n$. a small type of grasshopper. (4.469)

ha-mak, a-mak $n$. monkey, macaque. (4.418)

ha-mak rang-gol, ha-mak nang-gor, a-mak rang-gol $n$. long tailed monkey, langur, hanuman monkey. (4.418)

ha-ri, ha-ri-bo, a-ri-bo int. lets go!, an expression used to encourage the start of an activity. (0.64)

ha-rim-a, a-rim-a vi. late, delayed, a long time. (2.53)

ha-rong, a-rong $n$. heron, (grey heron), (Beng. sa-ros). (4.43)

ha-ti $n$. elephant. $<\mathrm{B}(4.419)$

ha-tur $n$. hammer. $<\mathrm{B}(5.838)$

ha-wa, a-wa dem. that far one, way over there. (1.5)

ha-wa-dang, a-wa-dang dem. those far ones, way over there. (1.5)

ha-wak-ha-wak grap-a vi. cry a lot, loudly, of a baby wanting to nurse. $(7.29)$

ha'-, $\mathbf{a}^{\bullet}-$, ha`-a, $\mathbf{a}^{-}-\mathbf{a}$, ha $n$. earth, ground, soil, land. ha*-, $\mathbf{a}^{\bullet-} c p$. categorizing prefix for land, soil, countryside: ha-kin-te lumps of earth; ha--ba fields; a'-gil-sak world. (4.242)

ha'-an-cheng $n$. soil mixed with sand. (4.242)

ha-ba, a'-ba $n$. agricultural land, land used for either wet rice cultivation or for slash and burn. (5.112)

ha'-ba git-dal, a'-dal $n$. first year slash and burn fields. (5.113)

ha'-ba hu'-a, a'-ba $\mathbf{o}^{\cdot}-\mathbf{a}$ vph. cultivate by slash and burn. (5.113)

ha-ba nok-dang, a-ba nok-dang $n$. land and house, property of a household, even of a poor household. (8.423)

ha'-ba song-ba, a'-ba song-ba $n$. property, (lit. fields and village). (0.7) ha-ba song-ba, a-ba song-ba $n$. property, (lit. fields and village). $(8.423)$ 
ha·-ba-ku, a•-ba-ku $n$. a width of field worked by one person moving along, as when planting rice. (5.114)

ha-ba-rim-rim, a-ba-rim-rim $n$. lots of fields, fields everywhere. (5.112)

ha'-bol, a'-bol $n$. firewood. (5.423)

ha'-bol bi-pek, a•-bol bi-pek $n$. small firewood. (5.423)

ha'-bol-nok, a·-bol-nok $n$. woodshed. (5.622)

ha-breng, a-breng, ha-beng $n$. slash and burn fields in their second year of use. (5.113)

ha-bri, a'-bri $n$. mountain, large hill. (4.232)

ha'-chik, a'-chik $n$. mountain, hill, upward slope as of a road. (4.232)

ha'-dal, a’-dal, ha•-ba git-dal $n$. first year slash and burn fields. (5.113)

ha-dam, a-dam $n$. location, place; place to sit; site, house site. (2.11)

ha-dam-san-o $l w$. together, in the same place. (2.11)

ha-dap-a, a-dap-a, dap-a vt. bury, as a body. (6.79)

ha-ding, a•-ding $n$. cultivatable land that is higher than its surroundings, land that does not flood. (5.112)

ha·-dip-ek, a•-dip-ek n. mud. (4.246)

ha·-dip-u, a•-dip-u $n$. ashes. (5.421)

ha'-dok, a'-dok $n$. place, region, area, location, such as a field, village, country. (2.11)

ha`-ga, a`-ga $n$. land that is slightly higher than its surroundings, land that does not flood; dry land, in contrast to a pond or lake. (4.232)

ha•-gal, a•-gal $n$. old abandoned slash and burn fields. (5.113)

ha-gat-e, a-gat-i, a-gat-e $a d v$. outside, open, untucked, of a shirt; classificatory rather than close kin, as a cousin-brother rather than a real brother. (5.644)

ha`-gi-sim, a`-gi-sim, ha•-gil-gi-sim, ha•-mang-gi-sim $n$. dark earth, such as earth of a field for wet rice, (lit. black earth). (4.242)

ha•-gil-sak, a•-gil-sak $n$. world, earth. (4.231)

ha•-gin-dil-a, $\mathbf{a}^{\bullet}$-gin-dil-a $n$. dust, particularly dust that is flying in the air. (4.248)

ha`-gip-ok, a`-gip-ok, ha·-gil-gip-ok, ha·-mang-gip-ok $n$. grey colored earth from rice fields used to smear on a house, (lit. white earth). (4.242)

ha•-gip-u-a, a`-gip-u-a vi. open up, of the ground in an earthquake. (4.37)

ha'-git-chak, ha'-mang git-chak $n$. the ordinary yellow to rust-brown soil found in the raised area of Modhupur; earth used to build roads, (lit. red earth). (4.242)

ha'-git-ting, a'-git-ting $n$. unburned ground, ordinary ground, in contrast to a fireplace; an area of a slash and burn field that escapes the fire, (lit. raw earth). (4.242) 
ha'-gop, a'-gop, gop-ang $n$. big hole, larger than $\mathbf{a}^{\cdot}$-kil-ok; low spot of land within a hilly area. (2.38)

ha-jak, a-jak $n$. reciprocal labor, labor to be paid back, labor exchange. $(8.433)$

ha'-jak ron·-a vt. give work for pay. (8.433)

ha'-ji-ri, $\mathbf{a}^{\cdot}-\mathbf{j i}$-ri $n$. abandoned slash and burn field, field that has reverted to forest, old dark jungle. (5.113)

ha-ka-ki, a'-ka-ki n. a kind of earth that lacks sand and that is hard when dry but very sticky when wet. (4.242)

ha'-keng-krak, a'-keng-krak $n$. hard clay. (4.242)

ha-kil-ok, a-kil-ok $n$. hole, specifically a hole in the ground but extended to any hole; nostril, ear hole; eye of a needle. (2.38)

ha-kim, a-kim $n$. relation between two lineages that is formed and perpetuated by marriage. (6.651)

ha-kin, a-kin $n$. type of white ant that builds large ant hills, and that bores into and lives in the mud walls of houses. (4.465)

ha-kin-chi, a'-kin-chi $n$. loose dust, the fine dirt that grinds off top of packed dried earth. (4.248)

ha-kin-chi re-wa-a vi. dirty from dust. (3.716)

ha-kin-te, ha-kin-ti, a'-kin-te $n$. lumps of earth that are bigger than rong-krek. (4.244)

ha-king, a-king $n$. the plot of land to which the members of a village had cultivation rights during the period of slash and burn agriculture, whose title was held by the man known as the ha-king nok-ma. (5.112)

ha-king nok-ma $n$. the man who held the title for the village land during the time of slash and burn agriculture. (8.12)

ha'-kol, a'-kol, hang--kol, ang-kol $n$. hole, in the ground or in any object. (2.38)

ha-kong, a-kong $n$. low place between hills that is wider than a ha'-sing-sep; valley. (4.232)

ha'-ma, $\mathbf{a}^{\cdot}-\mathbf{m a} n$. common land of a whole family, in contrast to the ha'-tot which is an individual's plot. (5.112)

ha'-mang, a'-mang $n$. soil, earth, what is dug out when digging a hole: ha'-mang ha'-mang dak-e so-eng-a smelling of soil, as water from a well may smell. (4.242)

ha'-nem-a vi. tasting of dirt that is mixed into food. (3.682)

ha'-ni, a`-ni $n$. earthen, made of earth, of houses: ha'-ni be-ra mud walls. (5.631)

ha'-ni be-ra, a'-ni be-ra $n$. earth wall. (5.638)

ha'-ning-gu-ang, a'-ning-gu-ang, gu-ang-ha'-ning $n$. a poisonous ground living spider. (4.464)

ha'-pa-o lw. on the ground, ground level, as opposed to upper stories. $(2.11)$ 
ha'-pal, a'-pal $n$. low land; flat land, land used for wet rice in contrast to slash and burn; specifically: the area of north Mymensingh where Mandis live. (4.231)

ha'-pek, a'-pek $n$. wet clay, mud. (4.246)

ha'-pru, ha'-pu-ru, ha'-pru-tot, a'-pru $n$. big anthill made by a'-kin ants. (4.475)

ha-ra-gat-a vt. build an earth platform such as a house platform, a veranda, a shelf, a storage area in the cooking room; pile up dirt. (5.637)

ha'-ran, a'-ran, ha--ran-ka-ri $n$. dry season, the time of dried earth. $(2.84)$

ha'-rang, ha'-drang $n$. ground, land. (4.231)

ha'-rik-a, ha'-rik-et-a vt. chase away, drive away: ma'-su-ko ha'-riking-a driving away cattle. (4.49)

ha'-rim-il-a, a'-rim-il-a $n$. slippery ground. (4.246)

ha-rong-ga, a'-rong-ga $n$. uplands, hills; specifically: the uplands of the Garo Hills. (4.232)

ha-rong-gi-rong-ga $n$. soil of mixed types, red, white, black. (4.244)

ha-rong-gra $n$. earth with small hard lumps, (same as rong-krek). $(4.244)$

ha-rong-krek $n$. dirt with small hard lumps. (4.244)

ha'-rong-rong, a'-rong-rong $n$. sloping land. (5.112)

ha'-sak-a, a'-sak-a $n$. high land, hill tract. (4.232)

ha'-sam $n$. side of a field. (5.87)

ha-san $n$. brick, dried mud brick. (5.636)

ha'-sel-o, a'-sel-o $p p$. because of, for the reason, (follows genitive -ni). $(1.13)$

ha'-si $n$. echo in ha'-kim ha'-si the relation between two lineages formed by a marriage. (6.651)

ha-sik-a, a-sik-a $v t$. want to, wish to, desire, (usually with an infinitive verb compliment): ko-bol-ko kin-a-na $\mathbf{a}^{\cdot}$-sik-a want to hear the news. (7.725)

ha'-sik-a-git-a, a'-sik-a-git-a $a d v$. according to one's wishes, on purpose, voluntarily. (7.725)

ha'-sik-en, a'-sik-en $a d v$. according to one's wishes, on purpose, (A•pal). (7.725)

ha'-sing-sep, $\mathbf{a}^{\cdot}$-sing-sep $n$. pass, low area between two hills that is narrower than a ha-kong. (4.232)

ha'-sol-sol, ha'-so-sol, a'-sol-sol $n$. slope of a mountain, slope down. $(4.232)$

ha'-song, $\mathbf{a}^{\cdot-s o n g} n$. country, nation, native place. (8.22)

ha'-stap, a'-stap, ha'-stap-a $n$. sticky mud. (4.246)

ha-ta-ru, a'-ta-ru $n$. deep muddy place, swamp. (4.253) 
ha'-ti, a'-ti n. market, week. (8.449)

ha'-til-a, a'-til-a $n$. courtyard, open area between the houses and buildings of a homestead. (5.644)

ha-ting-gak $n$. an upward slope, rise. (4.232)

ha-tot, a-tot $n$. land allotted to a single individual to use, often to a young person; property of an individual, including land, goods, rice, etc. $(5.112)$

ha'-wal, a'-wal $n$. all property rights, (lit. land and fire): ha'-wal wat-a to break the lineage bonds when divorce occurs, (lit. send away the land and fire). (8.423)

ha-we, ha-wi, ho-we, a-we, a-we, we, u-e, hu-e-ro, ho int. yes. $(1.24)$

hai, hai-bo, hai-ba-bo int. lets go!, an expression used to get action started. (0.64)

hai-brens, hai-bren-chi $n$. tall bench with a back. $<$ E (5.82)

hai-da int. I don't know, (A•chik). (1.24)

hai-gil-a, har-gil-a $n$. stork, large, gray, long-billed bird that eats fish and eels, as big as a so-gon vulture, (adjutant stork). $<$ B (4.43)

hai-wa, ai-wa dem. that distant one, the one way over there. (1.5)

hai-a, ai'-a vt. know, understand. (7.41)

hai-chak-a vt. anticipate, expect. (7.48)

hai--ja vi. (I) don't know. (1.24)

hai'-man-a ku'-man-a vph. clever, good at language. (0.7)

hai-rik-a vi. recognize. (7.45)

hak-a vi. cough up and spit. $<\mathrm{B}(9.44)$

hal, al $n$. set of cattle, yoke, plow, and plowman; team and equipment needed to plow. $<$ B (5.154)

hal we-a, al wi-a $v t$. plow, cultivate by the plow. $<$ B (5.154)

hal-a, al-a vt. to plow. $<\mathrm{B}(5.154)$

hal-i, al-i $n$. dike between rice fields. $<$ B (5.114)

ham-chak-a, am-chak-a vt. stand, endure, put up with; often used in the negative: ham-chak-ja can't stand. (7.56)

ham-sok-a, am-sok-a vi. confident, feel oneself to be capable; able to, manage, have the courage or patience for. (7.55)

ham•-a, am•-a vt. 1. want, desire, ask for, (usually with a noun object): ja-ba am-ing-a want curry. 2. be about to, on the point of, almost, (usually with a verbal compliment): si-na am•-ing-a about to die. (7.725)

ham-bru-a, am•-bru-a vt. pretend to want. (7.725)

ham-chak-a, am-chak-a vt. want on behalf of someone else. (7.725)

ham'-chel-a, am•-chel-a $v t$. want, demand, take by force. (7.36)

ham-chrak-a, am•-chrak-a vt. demand, take by force. (7.36) 
ham•-grik-a, am•-grik-a vt. want each other. (7.725)

ham•-pil-a, am•-pil-a vt. turn, turn away, turn around, turn over, reverse; stir; sweep rice into pounder hole. vi. turn over, as in bed. (9.327)

ham·-pok, am·-pok n. low stool. (5.82)

ham•-rik-a, am•-rik-rik-a vt. want more and more. (7.725)

han, han॰-, an·- $n$. body, usually used in compounds. (6.21)

han bi-bom $n$. having a round chest, round limbs. (6.48)

han bo-a vi. body swells up. (8.956)

han cheng-a, han'-cheng-a, han-jak cheng-a vi. healthy, feel good, feel light; slightly drunk, (lit. body-light). (8.91)

han chrim-a, han-chrim-a vi. feel bad, (lit. body-heavy). (8.91)

han dal-a vi. big, of a body. (6.44)

han jak, han•-o jak-o $n$. entire body, on the whole body: han•-jak nam-a healthy. (6.21)

han mil-a vi. fat, of bodies. (6.48)

han॰-, han, an॰- $n$. body, usually used in compounds. (6.21)

han-chak-a vi. endure, put up with, yield; take into the body, as a disease, get sick; become pregnant. (7.56)

han-cheng, an·-cheng $n$. sand. (4.242)

han'-cheng-a, an·-cheng-a, han·-jak cheng•-a vi. feel good, feel light, feel healthy, just barely drunk, (lit. body-light). (5.48)

han-cheng-bit, na·-cheng-bit $n$. a kind of edible fish that is smaller than a sardine. (4.454)

han'-chi, an·-chi $n$. blood, (lit. body-water). (6.29)

han-chon-a vi. small bodied. (6.46)

han-dal-a, han dal-a vi. big, fat. (6.48)

han'-dil, an`-dil $n$. bad odor of the body. (3.688)

han-dil so-eng-a vph. dirty of the body, unbathed, bad odor. (6.58)

han-ding-a vi. have a fever. (8.93)

han-gi-sim, an•-gi-sim n. dark complexion, (lit. body-black). (3.534)

han•-git-chak, an·-git-chak $n$. light complexion but light within the local range rather than pale like Europeans, (lit. body-red). (3.532)

han-jak nam-a vi. healthy, (lit. body-hand-good). (8.91)

han·-jrim-a vi. weak, sick; lazy, not feel like working, (lit. body-heavy). $(8.91)$

han'-o chi du-a vph. swell, of body parts. (8.956)

han-o ra•-a vph. deliver baby, as a midwife, (lit. take the body). (6.77)

han·-pak-a vi. abort spontaneously, miscarry. (6.77)

han-pel-dang $a d v$. having a body that is wide, but thin from front to back. (6.44)

han·-pi-a, an•-pi-a vt. stretch the body, as after sleeping. (9.319)

han-rang $n$. all body parts. (6.21) 
han'-sal-a, an`-sal-a vi. stretch the body, as after sleeping, (lit. bodypull). (9.319)

han'-sel-a, an'-sel-a vi. healthy of body. (8.91)

han'-seng-a, an'-seng-a vi. happy, joyful, dance, celebrate. (7.721)

han-seng-wil-wil-a $n$. dance joyfully in a circle, as in a kiir-ton. (8.83)

han-sim-mo-a vi. shiver, shake, from cold or fear. (9.322)

han'-srik-han'-srik, an'-srik-an`-srik $a d v$. nervous, slightly afraid. $(7.764)$

han'-tang, an'-tang, han'-tang-tang pro. self, selves, oneself, reflexive pronoun. (1.64)

hang-a vt. hold hands up to the fire to warm them; warm or dry objects such as cloth or paper by holding them up to the fire. (5.426)

hang-jang $a d v$. without reason, with no purpose, randomly, hurriedly. (3.44)

hang-ki, ang-ki $n$. crab. (4.458)

hang-ki ha--kil-ok, hang--kil-ok $n$. crab hole. (4.475)

hang-ki ma na-tik $n p h$. boy or girl? Question asked at a birth about the gender of the newborn baby, (lit. crab or shrimp?). (6.77)

hang-ki-sa-a, ang•-ki-sa-a vi. to have a skin ulcer, rash, open skin sore ulcer. hang-ki-sa, ang-ki-sa $n$. (8.953)

hap-pen, pen $n$. short pants, half pants. $<\mathrm{E}(6.527)$

har-mo-ni $n$. harmonium. <E (8.85)

he'-sal, hi-sal, ri-sal, i-sal n. banana leaf. (4.675)

he-sip-a, hi-sip-a $n$. banana leaves. (4.675)

hel-di, el-di, hol-di, ol-di $n$. turmeric. $<$ B (5.405)

hel--bu-a, el--bu-a vi. spit up a small amount, like a baby. (8.97)

hel-hel $a d v$. a little angry. (7.766)

hem-hem, hem-a-hem-a $a d v$. enough, sufficient; eaten plenty, done a lot, tired of: hem-hem cha-a-ring-a eating plenty. (3.341)

hen-del $n$. handlebars. $<\mathrm{E}(5.872)$

heng-a, eng-a vt. threaten to hit. (8.36)

heng-gok $n$. snore. heng-gok ra-a $v p h$. snore, (lit. take a snore). (7.96)

heng-a vi. woven loosely, with open spaces, of baskets, cloth. heng•-eheng-e, heng*-gri-heng-gri $a d v$. widely spaced, of the pieces of a basket. (2.25)

hi-jam-a, hi-jam-jam-a, i-jam-a, e-jam-a vi. yawn. (7.96)

hi·-a vi. go, an unusual variant of $\mathbf{i} \cdot \mathbf{- a}$, which see. (9.312)

hi-sal, he'-sal, i'-sal $n$. banana leaf. (4.675)

hil-hil $a d v$. lots, of people, animals: man-di hil-hil dong-a there are lots of people. (3.342)

hil--hil-a, il--il-a vi. loud noise, rumble of thunder, rumble like an engine. $(4.342)$ 
hil·-hil-tim•-tim-a vi. make a loud noise, like machinery, like a rice mill, airplane. (3.628)

hil·-im hil·-im $a d v$. in large numbers, of people, animals. (3.342)

hil•-im-hil•-im adv. flame up, burn strongly. (5.425)

him-a, im-a vt. hold something up to the fire to warm it or dry it, hands to warm, a pot to dry: te-rik bi-jak-ko him-a warm banana leaves at the fire (to soften them so that they do not break); press on, as heated medicinal herbs are pressed on on a sore spot; darken bamboo strips in smoke. (5.426)

him-him-a, him-him-grik-a vi. have sexual relations. (6.72)

hit-a, it-a, it-et-a vt. order, command. (9.75)

ho, ho•-gip-a $n$. father-in-law, (A·pal). (6.611)

ho-ri-gol $n$. wild pigeon, mixed green and yellow-colored bird that eats banyan fruit, (common green pigeon). <B (4.43)

ho-ri-ho-ri int. get away, move! $<\mathrm{B}(0.66)$

ho•-a, hu•-a, o*-a, u'-a, ha•-ba ho*-a vt. clear forest for slash and burn cultivation. (5.113)

ho-bit-e, hu·-bit-i, o'-bit-e $n$. father-in-law. (6.611)

ho--bit-e-sa $n$. father-in-law and son-in-law. (6.62)

ho-on-a, hong•-gon-a, o•-gon-a, ong•-on-a vi. get down, get off, from a chair, bus, ox cart, train; climb down, from a tree, mountain. (9.318)

ho•-pa-a, hu•-pa-a, o•-pa-a vt. clearing along with someone else, clearing together by slash and burn. (5.113)

ho-rut-chok-a, hu•-rut-chok-a $v t$. clear roughly with an at-te (bamboo knife), cut out weeds. (5.156)

hol-a, ol-a $v t$. hold or carry by a tump line. (9.532)

hol-di, ol-di, hel-di, el-di $n$. turmeric, turmeric colored, yellow. $<\mathrm{B}$ (5.405)

hon-dim-a, on-dim-a vi. short, low, near the ground, of a house, tree, post, hill; opposite of chu-a-a, chu-ak-a. (2.11)

hon·-a, on·-a, ron·-a vt. give, (see ron·- for compounds). (9.72)

hong•-a, ong•-a vi. be, exist, (see ong•- for compounds). (9.11)

hong•-gon-a, ong•-gon-a vi. get down, get off, from a chair, bus, ox cart, train; climb down, from a tree, mountain, (same as ho•-on-a). (9.318)

hong-kat-a, ong·-kat-a vi. come out, appear, emerge; climb down carefully and without jumping, as from a tree; come out, of skin diseases. (9.313)

hop-a, hu-ap-a vi. cry made by animals such as a barking deer. (4.476)

hu-hu-dok-dok $n$. dry season, when the ground is hard and dry. (2.84)

hu•-a, ho'-a, u'-a, $\mathbf{o}^{*}-\mathbf{a}$, ha•-ba ho'-a vt. clear land for slash and burn cultivation, (see ho'- for compounds). (5.113)

hul $n$. wooden side pieces of a yoke, to which the ropes are attached. $(5.87)$ 
hul-a $n$. dried jute stalks, often used for taking a fire from one place to another, firebrand. (5.423)

hul-a, ul·-a vt. sharpen, make sharp. (3.666)

-i pvs. subordinating suffix, having, being, (see -e). (0.216)

i-a dem. this: i-a song this village. it (close): $\mathbf{i}-\mathbf{a}$ dal-a it is big, this is big. this (for units of time): i-a bil-si-o this year; i-a ja-o this month; i-a sop-ta-o this week. i-, i-- combining form: i-ko nik-jok (I) saw this; $\mathbf{i} \cdot-n i$ gim-in because of this; $\mathbf{i} \cdot$-git-a dak-bo do like this. (1.5)

i-a int. an expression that fills time while gathering one's thoughts. (0.64) i-a wal-o lw. tonight. (2.63)

i-a-dang, i-a-drang, i-a-rang, i-dang, i-drang dem. these. (1.5)

i-a-no, in-o, i-a-o, i-o lw. here. (2.13)

i-am-a, re'-am-a vi. roam, wander, take a walk. (9.319)

i-dang, i-drang, i-a-dang, i-a-drang, i-a-rang dem. these. (1.5)

i-jam-a, e-jam-a, hi-jam-a, hi-jam-jam-a vi. yawn. (7.96)

-i-ming pvs. subordinating suffix, having, being, (see -e-ming). (0.216)

i-ni-ku-no, in-i-ku-no, hin-i-ku-no conj. then, moreover, in addition. $(1.4)$

i-o, in·-o, i-a-no $l w$. here. (2.13)

i-ri, i-ri mi $n$. a new variety of rice from IRRI, the International Rice Research Institute. $<\mathrm{E}(5.25)$

i-skul, skul $n$. school. $<\mathrm{E}(8.6)$

i-skul-nok, skul-nok $n$. school building. $<\mathrm{B}(5.624)$

I-sol $n$. the Christian God. <B (8.54)

i-song pro. they, (variant of bi-song). (1.62)

i-a vi. go, come, (same as re'-a). (9.312)

i-ang-a vi. go. (9.312)

i-ang-chek-a, i'-ang-sek-a vi. go ahead, pass by. (9.312)

i'-ang-jem--jem-a vi. go repeatedly. (9.312)

i-ang-jol-jol-a vi. go continuously, without stopping. (9.312)

i-ang-jring-a vi. go often, repeatedly. (9.312)

i-ang-pa-a vi. go along with, accompany. (9.312)

i-ang-pil-a vi. go back, return. (9.312)

i'-ang-sek-a, i'-ang-chek-a vi. go ahead, pass by someone. (9.312)

i-ang-srang-a vi. gone completely, gone for good. (9.312)

i-ba-a vi. come. (9.312)

i-ba-bru-a vi. come when not expected, at a bad time. (9.312)

i'-ba-pa-a vi. come with, accompany. (9.312)

i-ba-pak-a vi. come by way of, do on the way. (9.312)

i--ba-pil-a vi. come back, return to here. (9.312)

i-cha bak, i-cha-bak-a ppp. on this side, here. (2.13)

i-cha-i'-cha $a d v$. here and there. (2.13)

i-chik-chik dem. like this. (3.433) 
$\mathbf{i}$-ching, $\mathbf{e}^{\cdot}$-ching $n$. ginger. (5.23)

i-gong, e•-gong $n$. midrib of a banana leaf. (4.675)

i-king, e--king $n$. woman's skirt, wrap-around skirt. (6.526)

i-mik-brang-a vi. take the wrong road, get lost, go carelessly, go where one should not go. (9.538)

i-ni gim-in conj. therefore, for that reason; a semi-lexicalized form that acts like a conjunction. (1.4)

i-ni so-moi-o $a d v$. at this time. (2.61)

i-ni-ko, hi-ni-ko, i-ni-ko, in-i-ko conj. then, in addition, moreover. (1.4)

$\mathbf{i}-\mathbf{n o}$, hi-no, in-o, i-o conj. then, and then, at that time. (1.4)

i-no, in॰-o, i-a-no, i-a-o, i-o lw. here. (2.13)

i-rik-a, hi-rik-a, re•rik-a vi. follow, follow after, go behind. (9.328)

i-ru-ra•-a, i-ru-rat-a, hi-ru-rat-a vi. go back and forth, commute. $(9.315)$

i-sal, e•-sal $n$. banana leaf. (4.675)

i-tong-tang-a vi. go straight, directly, take a short-cut. (9.312)

iin-chi- cls. classifier for inches. $<\mathrm{E}(1.36)$

iis $n$. the front or forward pointing part of a plow. (5.87)

iis, i-si int. yuck, expression of disgust. (0.62)

iit $n$. brick. <B (5.636)

il-sik-il-dang adv. wiggle, not stay quiet. (9.324)

il·-il-a, hil·-hil-a vi. sound or rumble of thunder, rumble like an engine. (4.342)

im-a, him-a vt. hold something up to the fire to warm, dry, or darken in the smoke, as the hands to warm, a pot to dry, bamboo strips to darken. (5.426)

im-be-a vi. bleat of a calf. (4.476)

im-bing-gi, am-bing-gi $n$. reed of a sa-nai (small musical horn). (8.85)

im-bo-a vi. moo, sound made by a calf or cow. (4.476)

-in $n s$. foregrounding suffix, emphatic. (0.14)

in-a vi. say, it was said, when reporting another's speech; tell, order. $(7.12)$

in-bru-a vi. tell wrongly, (same as a-gan-bru-a). (7.25)

in-chak-a vi. reply, answer. (7.32)

in-chik dem. this much, like this. (3.433)

in-dak-a dem. in that way, like that. (3.433)

in-dak-e, in-dak-i, in-di-dak-e, hin-di-dak-e, in-dik-e conj. in this way, like this; this looks like a complex morphological form with an underlying meaning something like 'doing like this' but it has been well lexicalized. (1.4)

in-di-ba, hin'-di-ba conj. but, however: re'-ang-na ha-sik-a in-di-ba man•-ja (I) want to go but cannot. (1.4) 
in-di-o-ba hin-di-o-ba conj. but anyway, in spite of: dok-be-jok indi-o-ba grap-ja (he was) hit a lot but even so doesn't cry. (1.4)

in-di-sik, in-di-sik-sik, in-di-sik-ni dem. like this, this much. (3.433)

in-dik-e, in-dik-i, hin-dik-i conj. like this, in this way, (same as indak-e). (1.4)

in-et-a vt. tell, inform: ang-a nang-ko dok-no-a in-et-a I tell you (that) I will hit you. (7.12)

in-ga-gal-a vi. talk quickly, carelessly. (7.14)

in-git-chi-a $v t$. insult by saying something. (7.27)

in-grik-a vi. converse, often angrily; echo in ni-ri-a in-grik-a get along badly, quarrel. (8.32)

in-grik-bru-a $v t$. quarrel in jest. (8.32)

in-grim-a $v i$. talk at the same time; say the same thing. (7.12)

in-i-ko, hin-i-ko conj. in addition, moreover. (1.4)

in-jiil, in-jiin $n$. engine. $<\mathrm{E}(5.86)$

in-tat-a vt. insult. (7.27)

in'-cha-in'-cha $a d v$. here and there. (2.13)

in'-o, i-no, i-a-no, i-a-o, i-o lw. here, at that time. (2.13)

-ing- $a a$. progressive, indicates action in progress or a continuing state: grap-ing-a crying; bu-ing-a telling lies, joking, (A•chik: -eng- ). (0.41)

it-a, it-et-a, hit-a vt. order, command. (9.75)

ja $n$. month. (2.53)

ja re'-a, ja-na re'-a vph. menstruate, (lit. month goes). (6.72)

-ja- $a a$. no, not, negative particle: ding-ja not hot; grap-ja does not cry. (0.41)

ja- cls. classifier for months. (1.33)

ja-ba $n$. curry, side dish eaten with rice. (5.409)

ja-brang-a, gi-sik ja-brang-a vi. terrified. (7.764)

ja-but-chum $n$. dark of the moon, time before the new moon, (lit. old lady moon). (4.214)

ja-cha-a vi. disappointed, unsatisfied. (7.742)

ja-da $n$. stupid man, fool, insane man. $<$ B (7.44)

ja-da su-brok $n$. an insult for stupid people. (7.27)

ja-di $n$. stupid woman, incompetent woman, insane woman. $<\mathrm{B}(7.44)$

ja-din-a vi. appear in a dream: ang-o Ne-sen-pa ju-mang-cha jadin-a I saw Ne-sen-a in a dream, (lit. Ne-sen-pa appeared to/at me in a dream). (7.96)

ja-gip-u-a vi be startled, jump with fright, as when hearing a sudden noise. (7.763)

ja-gok-a vi. shout out with fear. (7.763)

ja-gring $n$. shadow, reflection in mirror or water. (3.558)

ja-ja-a vi. hesitate, make mistakes, speak with errors, speak forgetfully. $(7.25)$ 
ja-jong $n$. moon. (4.214)

ja-jong or-dek, ja-or-dek, ja-ni or-dek $n$. quarter moon. (4.214)

ja-jreng-a vi. be worried, a little frightened. (7.764)

ja-ju-mang, ju-mang $n$. dream. (7.96)

ja-ka-ri echo. in ja-ju-mang ja-ka-ri dream. (7.96)

ja-kam-bi $n$. quarter moon. (4.214)

ja-mai $n$. bridegroom, son-in-law. <B (6.617)

ja-min-ok-a, ja-jong min-ok-a, ja-ko min-ok-a vph. eclipse of the moon; be stupid, (lit. swallow the moon, from the fable that an eclipse comes when a monster swallows the moon). (4.214)

ja-na re'-a vi. menstruation, (lit. go for the month). (6.72)

ja-na-la $n$. window. $<$ B (5.638)

ja-nap-a vi. inform. (7.34)

ja-ra-gat-a $v i$. the return procession from the grave after a burial, or from the site of a sacrifice, which may include drum or other music and dancing. (8.562)

ja-ram-bong $n$. full moon. (4.214)

ja-ri-a vt. shake with a circular rice tray and with a circular motion, to sort and sift grain. (5.451)

ja-ro-bi-a $a d v$. month after month, a long time. (2.53)

ja-rul $n$. tree with pale trunk, leafless in January but with little round fruit and beautiful flowers. $<$ B (4.632)

ja-seng-a vi. light, opposite of dark, as a room with windows; clear up, become light, as from lamps or at dawn. (3.554)

ja-wa pro. someone else; ja-wa-ni someone else's; ja-wa-na for someone else. (1.64)

ja·- $n$. leg, foot, usually in compounds. $c p$. prefix for parts of the legs and feet: ja·-git-ok ankle. (6.237)

ja-bak $n$. a carefully piled and orderly stack, of firewood, thatch, etc. $(5.423)$

ja-bik $n$. a severe sore on the sole of the foot. (8.953)

ja-bing-bang-a vi. stumble. (9.326)

ja·-bo $n$. swollen foot. (8.956)

ja·-chan-a vi. echo in $\mathbf{j a} \cdot \mathbf{- r i k - a}$ ja•-cha-na follow another person. (9.328)

ja·-chim-it-a vi. asleep, of legs, pins and needles. (3.64)

ja-chok $n$. entire leg, upper and lower; leg of bird; one of the four vertical side pieces of a basket; foot treadle of a teng-ki (rice pounder). (6.237)

ja-chok kong•-krot-e chu-a vph. sleep with legs curled up. (9.343)

ja'-chok ku-mil-ong $n p h$. bare legged. (6.552)

ja-chok-gi-ro $n$. ankle bones. (6.238)

$\mathbf{j a}^{\bullet}-\mathbf{c h o l} \mathbf{k a} \cdot \mathbf{a}$ vph. agree, esp. about marriage arrangements and marriage replacements. (6.652) 
ja-chrang-chrang $a d v$. stand with knees sharply bent, but not down to a deep squat. (9.346)

ja'-chri-a, ja'-chi-ri-a, ja'-si-ri-a vi. stand on tiptoe, walk on tiptoes. (9.47)

ja-dil $n$. root; the part of the root that is above ground; tendon of hands and feet. (4.678)

ja'-dil, jak-si-ni ja`-dil, ja'-si-ni ja`-dil $n$. tendons of fingers or toes. $(6.235)$

ja'-dil ja•-si, ja`-dil-ni ja•-si $n$. small roots, (lit. root-toes). (4.678)

ja'-dil-ma $n$. large roots. (4.678)

ja-ding-a vi. limp. (8.96)

ja-dok-a vi. break apart, of old posts or dying trees, after being eaten by ants, etc. (9.282)

ja-dot-chi $n$. the bone at the side of the hip, the head of the femur. (6.243)

ja·-gam $n$. step; footprint, tracks. ja'-gam- cls. classifier for steps, foot prints. (6.238)

ja'-gam ga'-a $v p h$. take steps. (9.319)

ja-gang-a, ja'-gang-e dong-a vi. sit or stand with knees or legs spread apart. (9.349)

ja·-gek cha-ing-a $v p h$. cracks open between the toes. (8.953)

ja-git-ap-a vi. with crossed legs, of one person when sitting, or two people in bed. (9.349)

ja-git-ok $n$. ankle, (lit. leg-neck). (6.238)

ja-git-ok-gi-ro $n$. round ankle bones at the sides of the ankles. (6.238)

ja-git-ot-a vi. stub toe. (9.326)

ja'-git-teng $n$. leg; leg of a bird; large leg of an insect, (same as ja`-chok). (6.237)

ja-gok-a vi. die, of a tree; weaken, of a post. (9.282)

ja-gra $n$. lowest bamboo supports, running lengthwise, of a platform for a bu-rang (field house), that lie under and hold the dam-beng. (5.639)

ja'-gra, ja'-gra-sa, $\mathbf{j a}^{\cdot}$-ga-sa, ja'-ga $n$. snare, a loop trap that catches an animal's leg. (5.845)

ja·-gu-a vi. stumble into a hole and fall; do something accidentally that is undesirable. (9.326)

ja-ka-bok $n$. condition in which patches of the skin are depigmented, leukoderma. (8.953)

ja-kep-a $v t$. hold pinched between one's feet. (9.531)

ja-kim-il $n$. hair on legs. (6.25)

ja-kin-chok $n$. heel, (A•pal). (6.238)

ja-kol $n$. footprint, hole left by foot. (6.238)

ja-kom-peng $n$. crippled leg. (6.237) 
ja-kong-krang $n$. ankle ornament with bells. (6.564)

ja-kop $n$. sandal, shoe, wooden sandals. (6.523)

ja'-ku re'-a vi. go on foot, walk. (9.319)

ja-ku-a $n$. first row of thatch at the edge of the eaves. (5.632)

ja-ku-ak $n$. back of knee. (6.237)

ja'-man, ja'-man-o $p p$. after, afterward, (follows genitive -ni): cha'-a-ni ja'-man-o after eating. (1.12)

ja'-man-ja'-man $a d v$. one following after the other. (2.14)

ja'-man-ni a-ma, a-pa $n$. stepmother, stepfather. (6.612)

ja'-mik, ja'-mik-gi-ro $n$. ankle bones. (6.238)

ja'-min-tal-a vi. asleep, of legs. (3.64)

ja-neng-a vi. tired, of the legs, as from walking. (7.94)

ja-pa $n$. foot; sole of foot; the foot treadle of a rice pounder. (6.238)

ja-pa bret-a vph. cracked sole of the foot. (8.953)

ja'-pa ku'-chok bret-a vph. cracked heels. (8.953)

ja'-pa-gi-ro, ja'-chok-gi-ru $n$. round ankle bones. (6.238)

ja-pa-jang-gil $n$. top of foot, (lit. back of sole). (6.238)

ja-pa-ku'-chok $n$. heel. (6.238)

ja-pa-rip-rip $a d v$. with cloth covering down to the feet. (6.53)

ja-pak $n$. inside of the upper leg. (6.237)

ja-pang $n$. base of a mountain, place near the foot of the mountain.

ja'-pang, ja'-pang-o $p p$. near, beside, at the base of, (follows genitive -ni). (4.232)

ja'-pang-ja'-pang $a d v$. beside one another, in the nearby area. (2.25)

ja-pat-ti $n$. lower leg, esp. the back of the lower leg, calf. (6.237)

ja'-pil-dem•-a vi. cross legged, sit with legs folded. (9.345)

ja-ping $n$. thigh, upper leg, thigh of a bird. (6.237)

ja-ping bol-ma $n$. the thick upper part of the thigh, especially a large one. (6.237)

ja'-ping-tong $n$. upper part of the thigh. (6.237)

ja・-rat-a $v t$. transplant, esp. for trees. (5.14)

ja-rek $n$. edge of mountains, foot of a mountain, base of a mountain. $(4.232)$

ja•-rik-a vt. follow behind another person. (9.328)

ja-rik-i $n$. echo in bi-sim-ol ja--rik-i rice that ripens late and that is left at the first harvest to be collected later. (4.692)

ja-rik-ing $n$. shin, front part of the lower leg, (lit. leg-edge). (6.237)

ja-rim-a vi. long of pant legs, sleeves; large, of clothes. (3.341)

ja-rip-rip $a d v$. walk slowly but without resting. (2.724)

ja’-ro-a, ja'-ru-a vi. long, of pant legs, lunggis, shirttails; for a long time.

$(2.213)$

ja`-sam-o $p p$. beside, (follows genitive -ni). (1.11) 
ja'-si $n$. toe. $\mathbf{a}^{\cdot}$-bol $\mathbf{j a} \mathbf{a}^{-} \mathbf{s i} n$. small bits of firewood, (lit. toe-wood). (6.238)

ja-si git-ok chot-a vi. cracks under the toes, athlete's foot. (8.953)

ja'-si-bi-ma $n$. big toe. (6.238)

ja'-si-ja'-dil, ja'-si-ni ja'-dil $n$. tendons of the toes, (lit. toe-root). (6.238)

ja'-si-ri-a, ja'-chri-a, ja'-chi-ri-a vi. on tiptoe. (9.47)

ja-sim-il-a, ja-sim-il-e dong-a vi. sit with legs stretched out in front, either crossed or uncrossed. (9.345)

ja'-sin $n$. next younger sibling. (6.64)

ja·-skil $n$. toenail, claw. (6.238)

ja'-sku n. knee. (6.237)

ja'-sku-gi-ro, ja'-sku-gi-la $n$. kneecap. (6.237)

ja-sku-ting-a vi. kneel, sit with knees on the ground; poke with the knee. (9.349)

ja'-srang $n$. children's ankle bells. (6.564)

ja-sru-a $v t$. injure by means of a mon-tro (chant) that can kill or cause blindness. (8.566)

ja'-su-a vi. wash one's feet. (6.57)

ja'-su-et-a $v t$. wash someone else's feet. (6.57)

ja'-teng, ja'-teng-ga $n$. leg, esp. the leg from the knee down. (6.237)

ja-teng ga'-a vi. take steps. (9.319)

ja-ti $n$. notches on a log used as steps. (5.636)

ja-ting $n$. base of a tree, banana plant, etc.; base of a mountain; stubble after rice grain is cut; back end, of a pen, etc. (4.678)

ja-ting-rik-i $n$. bottom to top, whole thing. (2.11)

ja-wek-a vi. short legged, not tall, having big calves of the legs, regarded as ugly. ja-wek-wek dak-a $a d v$. (6.46)

-jai ts. contraction of negative -ja and emphatic -ai: i-a bol-de dal-jai this tree isn't big! (0.314)

jai-ga, ja-ga, ja-gai $n$. place, location. $<\mathrm{B}(2.11)$

jak $n$. hand, arm. jak- $c p$. prefix for parts of the hands and arms. jakcls. classifier for leaves: $\mathbf{i}$-sal jak-sa one banana leaf. (6.233)

jak no-gip-a $n$. midwife. (6.77)

jak stik-a $v t$. fold hands. (9.46)

jak-a-si $n$. left, left hand, the opposite kin group to one's own: jak-a-sicha bak, on the left side. (2.11)

jak-am-, jak-em- cls. classifier for the amount held in a closed hand. $(1.36)$

jak-brak $n$. inside of the upper arm, armpit. (6.233)

jak-chak-a vi. fit in the hands; manage to do; have time for; often in negative: jak-chak-ja busy, doesn't fit into hands, have no time for. $(7.55)$ 
jak-chi-ni jak-si $n$. any of the three middle fingers. (6.235)

jak-chik-su-a $v t$. poke with the elbow. (9.511)

jak-chim-it-a vi. asleep, of arms. (3.64)

jak-chon-a vi. small, of leaves. (2.211)

jak-chrok-a vi. with arms close to the sides of the body or folded closely on the chest. (9.349)

jak-gam-bu $n$. forearm. (6.233)

jak-git-ok $n$. wrist, (lit. arm-neck). (6.235)

jak-jip-a vi. beckon, wave, with palm downward, (lit. hand-fan). (9.46)

jak-ka-sin-a vi. work slowly. (2.724)

jak-kal-a $v t$. use. (9.58)

jak-kal-to-a vi. good to use, comfortable to use. (9.58)

jak-kep-a $v t$. hold pinched in nearly-closed fist. (9.531)

jak-kip-a vi. pinch the fingers tightly against the palm. (9.531)

jak-kom-peng $n$. a crippled arm. (6.233)

jak-ma $n$. technique, skill; the bad skill of a thief. (7.55)

jak-ma gam-u $v p h$. working with hands, work hard, not lazy, as at basket making, gardening, etc. (8.433)

jak-ma-jak-ma $a d v$. big, of leaves, for more than one. (2.211)

jak-mil-ong, jak-kim-il-ong, jak-mil-ong $n$. bare arms, without a bracelet, with short sleeves. (6.552)

jak-min-tal-a vi. asleep, of arms. (3.64)

jak-mit-chi $n$. pulse, as felt in the wrist. (6.233)

jak-neng-a vi. tired, of the hands. (7.94)

jak-pa $n$. palm of the hand; end of an insect leg. (6.235)

jak-pa bri-a vph. shake hands. (9.46)

jak-pa dok-a vi. clap hands. (9.46)

jak-pa-jang-gil $n$. back of hand, (lit. palm-back). (6.235)

jak-pak $n$. inside of the upper arm. (6.233)

jak-pang-pil $a d v$. with hands held behind the back. (9.349)

jak-pong, jak-prong $n$. upper arm. (6.233)

jak-ra $n$. right-hand; first, oldest child, first rice beer from a batch. (2.11)

jak-ra-cha bak $p p p$. on the right hand side. (1.11)

jak-ra-ra $a d v$. empty, empty handed; not pregnant. (2.26)

jak-rak-a vi. quick, fast, esp. of work with the hands; quick at eating. $(2.726)$

jak-rim-ra $n$. railing, as on a bridge, (lit. hand-hold). (5.646)

jak-ro-a, jak-ru-a vi. long, of shirt sleeves. (2.213)

jak-sam $n$. five taka, (lit. one hand). (8.447)

jak-sel-a vi. echo in an-sel-a jak-sel-a healthy. (8.91)

jak-si $n$. finger; distal segment of an insect leg. (6.235)

jak-si dal-gip-a nph. thumb. (6.235) 
jak-si ge-dok $n$. six fingers, having six fingers on one hand, said to occur among Bengalis but not Mandis. (8.96)

jak-si-a-ma $n$. thumb, (lit. mother-finger). (6.235)

jak-si-bi-ma $n$. thumb, (lit. female-finger). (6.235)

jak-si-chon-gip-a $n$. little finger. (6.235)

jak-si-gi-ro $n$. joints of fingers. (6.235)

jak-si-jang-chi, jak-si-ma-jang-chi $n$. middle finger, ring finger. (6.235)

jak-si-ku-ti $n$. tip of finger. (6.235)

jak-si-ot-a $v t$. point to, point out, show. (3.516)

jak-si-ot-ra $n$. index finger. (6.235)

jak-si-pi-sa $n$. little finger. (6.235)

jak-si-tam $n$. finger ring. (6.564)

jak-sil $n$. tendrils of plants. (4.671)

jak-sil $n$. a part of a kim-a (memorial post) that encircles the post. (8.58)

jak-skil $n$. fingernail, claw. (6.235)

jak-sku $n$. elbow. (6.233)

jak-sku $n$. elbow. (6.235)

jak-sram-a vi. work rapidly, able to get a lot done. (7.55)

jak-su-a vi. wash hands. (6.57)

jak-su-a vt. injection, (lit. arm pierce). (8.99)

jak-tom $n$. fist. (6.235)

jak-tom-a vi. make a fist. (9.46)

jak-tong $n$. forearm, lower part of forearm, wrist. (6.233)

jak-wat-a vt. let drop from hand; let go of: ang-a ko-lom-ko jak-wat-a

I let the pen slip and fall. (9.54)

jal, jal-i $n$. fish net. $<$ B (5.843)

jal-a $n$. rice seedling before transplanting. (4.677)

jal-a vi. increase in numbers, spread; swarm, of insects such as lice that come in large numbers; crowd, of people. (3.342)

jal-a go-a $v p h$. broadcast rice seed in a seedbed for growing jal-a (rice seedlings). (5.14)

jal-a-ko ru-a-sik-a vph. transplant rice seedlings. (5.14)

jal-ang-ka, jang-gil-a $n$. a frame to hold climbing plants. (5.835)

jal-pret jal-ro-ro-a vi. lots of people, children, kinsmen; crowded. (3.342)

jal-ang $n$. narrow foot bridge made with a log or pieces of bamboo. (5.646)

jal-ik $n$. chili pepper. (5.23)

jal--ik bal-ju-ri $n$. a long variety of chili pepper. (5.23)

jal-ik do-bi-ma $n$. a large round variety of chili pepper. (5.23)

jal-ik do-bi-ma gi-sim $n$. a black fruited chili pepper. (5.23)

jal-ik do-mi $n$. a variety of chili pepper with long fruit. (5.23) 
jal-ik man-di $n$. a variety of chili pepper, said to be found in A•rongga but not in A-bima. (5.23)

jal--ik mi-sek-ki $n$. a small and strong variety of chili pepper. (5.23)

jal--ik na-reng-ku-la $n$. a variety of chili pepper. (5.23)

jam $n$. granary, storehouse. (5.622)

jam $n$. tree with light, much incised bark and twisted trunk and branches: a small variety has willow like leaves, a larger variety has $15 \mathrm{~cm}$. dark green leaves, edible fruit in July. $<$ B (4.632)

jam-ap $n$. echo in nok-kap jam-ap house site, plot. (5.644)

jam-bu $n$. larger variety of jam tree. $<\mathrm{B}(4.632)$

jam-bu-ra $n$. pomelo, a large citrus tree and fruit. (5.22)

jam-ti-ti $n$. smaller variety of jam tree. $<$ B (4.632)

jan-ti $n$. central tube of a rice beer pot through which beer is strained. (5.438)

jang-a vi. make a pleasant sound, as a drum, bell, gong, voice; good speaking. (3.624)

jang-a-tang-a, jang-be-tang-be-a vi. noisy, be noisy. (3.628)

jang-be-a vi. very loud, noisy. (3.628)

jang-chi $n$. middle, middle portion of an object, such as a glass or a wall; halfway; body of an object: song jang-chi-o in the middle of the village. -jang-chi dns. middle: wal-jang-chi midnight; nok-jang-chi center of the house; bon-jang-chi-a end in the middle, when half done; ma--jangchi middle sized tiger; jak-si-jang-chi middle finger, ring finger. (5.87)

jang-e, jang-i $a d v$. loud, disturbing, of pigs, loudspeakers. jang-e bol-a, jang-i bol-a $v p h$. speak loudly, as when under the influence of rice beer. (3.628)

jang-gi $n$. life, soul, vital essence of a person. (8.52)

jang-gi $n$. trap; snare for catching birds; any device for catching animals, net, glue, or a covered hole. (5.845)

jang-gi gal-a $v p h$. die, (lit. throw away the jang-gi soul). (6.79)

jang-gi sik-et-a, jang-gi sik-a, jang-gi ron-a $n$. put in life, give life or the soul, in creating a human being, by a spirit or God. (8.52)

jang-gil $n$. back, back of body, back of an insect; back side of an object; green outer edge of bamboo; bottom of a banana leaf. (6.243)

jang-gil bol-gru, jang-gil-ni bol-gru $n$. backbone. (6.28)

jang-gil tik-a vi. turn one's back; with one's back toward something. (9.349)

jang-gil tik-rik-e chu-a $v p h$. sleep back to back. (9.343)

jang-gil-a, jal-ang-ka $n$. a frame to hold climbing plants. (5.835)

jang-gil-o, jang-gil-cha $p p$. behind, in back of, (follows genitive -ni), (A·chik). (1.11)

jang-ting-a vi. loud, make a loud noise. (3.628) 
jang-jot-a, jang--jot-jot-a vi. narrow, narrower at the middle than at the ends; thin waisted. jang-jot, jang--jot-jot $a d v$. (2.313)

jang-ki $n$. stairs, an attached ladder such as the ladder leading up to watchhouse; the notched bamboo placed so that a mit-e (spirit) can use it to climb to an altar. (5.636)

jap-a, jap-et-a vt. put one on top of the other, pile up, of cloth, blankets. (9.647)

jap-ak- cls. classifier for armsful, as leaves, firewood, etc. (1.36)

jap-u-jap-u $a d v$. plentiful, esp. of leaves or clothes, or of things in piles. $(3.342)$

jat $n$. group, ethnic group, tribe; caste; species, kind, variety. $<\mathrm{B}(6.89)$

-jat-chi, -jat-chi-o dns. middle: sal-jat-chi-o middle of the day; waljat-chi-o middle of the night. (0.16)

jat-ska $n$. belonging to one's own group. (6.89)

jat-ta $n$. spear; stiff broom for sweeping outside. (5.845)

jau $n$. tree with inedible fruit but planted by people because they find it pretty. $<\mathrm{B}(5.22)$

jau--jau-a vt. speak to others without much care, as at a drinking party. $(7.14)$

je pro. relative pronoun, that which, whatever, whoever: je-ko whatever, whomever (accusative); je-cha, je-o, je-no wherever; je-ni whosever; jegit-a somehow, however, in whatever way; je-sik-ni to whatever extent. $<\mathrm{B}(1.64)$

je so-moi-o $l w$. whenever, at whatever time. $<\mathrm{B}(2.71)$

je-a, ji-a $v t$. criticize, insult, say bad things about, dislike. gro je-a vi. make arguments, talk at a legal meeting. (7.27)

je-chak-a, ji-chak-a $v t$. refuse, deny. (7.33)

je-mon, je-mon-je conj. for example, for instance. $<\mathrm{B}(1.4)$

je-was $n$. wife's older sister, (same as bo-ji). $<\mathrm{B}(6.615)$

-je-et-, -jet- $a a$. heavily, with pressure: sik-jet-a press down hard, squash; on-jet-a put something heavy on top of something else. (0.46)

je-et-je•-et dak-ing-a, on-jet-jet-a $v t$. place something heavy on, press hard. je-et-je-et $a d v$. heavy, tight, of shoelaces, thatch, of a fully inflated football. -je·-et-, -jet- $a a$. heavily, with pressure: sik-jet-a press down hard, squash; on-jet-a, on-jet-dap-a put something heavy on top of something else. (9.67)

jek-jak-a vi. make noise, make a disturbance, as by children or when everyone talks together; talk a lot, as when drunk; talk at the same time. (3.628)

jek-jek-a vi. rain long but lightly. (4.327)

jek-ju-jek-ju mo-jim-a vph. shake back and forth, as in an earthquake or in a shaking tree. (9.322) 
jem·-jem-a vi. regularly, repeatedly, often: jem·-jem-a-ri bol-ing-a always talking. jem--jem $a d v .:$ jem•-jem i-jam-ing-a repeatedly yawning. -jem-jem- aa. repeatedly constantly: kat-jem-jem-a run a lot; dok-jem-jem-a hit constantly; i-ang-jem•-jem-a go repeatedly. (2.71)

jeng $n$. leaves, grass and small plants growing close to the ground, especially when used as food for cattle. (4.66)

jeng-gim $n$. a plant that grows in water at the ends of rice fields, taller than a man and of finger thickness. Used to make fences. (4.652)

jeng-grang $n$. large and rough openwork basket for leaves, etc. (5.852)

jep $n$. pocket. $<\mathrm{B}(6.521)$

-jep- aa. cover: rim-jep-a cover with the hand; ga--jep-a cover with the feet. (9.62)

-jep-jep-, -je-jep- $a a$. hard, forcefully, into pieces: ga--je-jep-a step with pressure, stamp on; dok-jep-jep-a keep hitting, break into small pieces; chik-jep-jep-a chew hard, chew into pieces. (3.72)

-jet-, -jet-jet- $a a$. hard, forcefully: ga'-jet-a, ga-jet-jet-a grab, hold tight, with feet or legs; on-jet-a, on-jet-dap-a put something heavy on top of something else; sik-jet-a, sik-jet-jet-a push hard, press hard. $(3.73)$

ji-bon $n$. life, lifetime. $<\mathrm{B}(6.77)$

ji-chak-a, je-chak-a $v t$. refuse, deny. (7.33)

ji-ga $n$. a flowering tree, leafless in winter but with red or green bulbous hard fruits at end of the branches. $<$ B (4.632)

ji-ga-a-gu-ru $n$. a red variety of ji-ga tree. (4.632)

ji-ga-du'-mi $n$. a variety of ji-ga tree. (4.632)

ji-niis $n$. thing, things, objects. $<\mathrm{B}(5.81)$

ji-ip-ji-ip $a d v$. good quality, of cloth, shirt, lunggi, etc. (6.598)

jiin-jiil $n$. door chain. $<\mathrm{B}(5.638)$

jiip $n$. car, auto. $<\mathrm{E}(5.86)$

jiit-ti-a, jit-ti-a vi. win, as at sports. $<$ B (8.81)

jik, jik-gip-a, jik-tang, ang-jik $n$. wife. (6.615)

jik gal-a, jik-ko wat-a vt. divorce, (lit. throw away one's wife). (6.655)

jik-chol $n$. the women of a man's wife's lineage, (same as nok-chik). (6.631)

jik-git-e, jik-git-i $n$. younger wife, second wife. (6.615)

jik-jik $a d v$. rain heavily: mik-ka jik-jik on-ing-a rain is falling heavily. $(4.327)$

jik-jik-a vi. noise of smacking made by babies at the breast. (3.624)

jik-jik-a $v t$. shake, as a fish line, a gourd when straining rice beer; bounce a child in play, tickle. (9.515)

jik-pang-ma $n$. first wife, senior wife, as opposed to jik-git-e. (6.615)

jik-se $n$. wife and husband, couple. (6.62) 
jik-sek-a vt. marry by starting to live together without formal steps; steal a wife; take someone else's wife. (6.651)

jik-skang $n$. first wife, former wife. (6.615)

jil-a vt. care for, support, have responsibility for children, old people, animals. (9.73)

jil-jil-a vi. shake, tremble, from illness or cold. (9.322)

jil-ma $n$. crowd, lots, of people, animals, not used for inanimate things. -jil-ma ns.: a-chak-jil-ma a lot of dogs. (3.342)

jil-au-a vi. lightning; flair up, of a fire. (4.344)

jil--jeng jil--jeng $a d v$. shiny, flickering, of a TV screen, pig fat, beautiful cattle. (3.556)

jim-a vi. dissolve, as salt in water, candy in the mouth; soak, melt, disappear. (9.279)

jin-ang $n$. loan, of money, rice, etc.: jin-ang bot-a make a loan for interest, as of rice or money, lend; jin-ang chot-a pay back a loan; jinang ra-a take a loan, borrow; jin-ang sro-a take a loan, borrow. (8.446)

jin-ong-a vi. separate from a liquid; settle out of water; rise to the top, as cream or fat; collect in one place. (9.279)

jing-jeng-ga-jeng $a d v$. shake, swing back and forth, of curtains, a flexible bridge, a plant frame. (9.218)

jing-jing-a vi. hang by the hands or the knees. (9.349)

jing-ka $n$. a long striped vegetable used in cooking. $<$ B (5.23)

jing:-jang-a vi. flash, of lightning; shine, of metal or a mirror. (4.344)

jing-jet-jing•-jet $a d v$. flicker, as reflecting metal, jewelry, a TV screen. $(3.556)$

jing-jing-a $v t$. shake something, as a circular rice tray when sorting rice; shake a finger, fist, or knife to threaten. (9.515)

jip-a vt. fan; fan drying rice to blow away the chaff; flap wings. -jip- $a a$. with fanning, flapping: grang-jip-a flap wings slowly, soar; jak-jip-a beckon, wave. (9.515)

jip-u, jip-u-jip-u adv. messy, piled up this way and that, of cloth, leaves. (3.718)

-jit- $a a$. move: ka-jit-a move something that is tied, especially an animal; de-jit-a move, put in a different place. (9.511)

jit-a vi. move, shift one's location, as to give someone room to sit; change residence. aa move: ka-jit-a move something that is tied, especially an animal; de·-jit-a move, put in a different place. (9.319)

jit-pak-a $v t$. shove a thing or a person to make it move. (9.511)

jo-ba-pul, jo-ba-bi-bal $n$. hibiscus. $<\mathrm{B}(5.28)$

jo-bra-a vi. mildly sick. (8.91)

jo-di conj. if. $<$ B (1.4)

jo-jong $n$. little brother, baby brother, affectionate name for a baby boy. $(6.614)$ 
jo-mi-dar $n$. landlord. $<\mathrm{B}(8.41)$

jo-ra-, ju-ra-, jur cls. classifier for pairs, teams of animals. $<\mathrm{B}(1.35)$

jo-ton ka-a vt. try, attempt. (7.725)

jo-a $v t$. fry, without water but sometimes with oil, as meat, cakes, cracked rice, etc. (5.46)

jo-kram-a, jo-kam-a vt. stir up curry when frying; fry, of meat, fish, cakes, etc. (5.46)

jo--ol-a, jol-a vi. flow, of water; rise or fall, as when water covers roads in the rainy season, recede, of flood water; ooze, as from the ear; run, of the nose. (8.93)

jo--ong $n$. bug, insect, $(\mathrm{A} \cdot$ chik $)$, (see jong•- for Mandi compounds). (4.461)

jo-rep-rep-a, jo•-re-rep-a vt. stir in extra bits, such as onions, to add flavor when frying. (5.46)

joi-pol, jol-poi, joi-na n. nutmeg tree. $<\mathrm{B}(5.22)$

Joi-sto, Jo-sto, Jo-sti $n$. a Bengali month that overlaps May and June. $<\mathrm{B}(2.822)$

jok $n$. water pitcher, jug, usually aluminum. $<\mathrm{E}(5.436)$

-jok pvs. indicates a change of state, for an action or condition that happened in the past but whose results continue in the present: kat-jok has run; kat-ja-jok does not run any more; ga-ak-jok fell, has fallen. $(0.211)$

jok-a vi. escape, get loose, get out, by people, animals, water, things. ma-rang jok-a vt. be rid of ma-rang (taboo, pollution). (9.313)

jok-et-a vt. let loose, allow to escape. (9.572)

jok-jek-a vt. shake, as medicine, a tree branch. (9.515)

jok-jok-a vi. shake, bounce, as when riding in a bus or rickshaw; shake jerkily, bounce in walking; bounce, as a child in play; bounce up and down on a tree branch. jok-jok, jok-jok-chi-chi $a d v$. (9.322)

-jok-ming $t s$. a pair of suffixes that combine to indicate a time previous to some other time, had: ching-a re-ang-jok-ming we had gone. (0.312)

-jok-o, -jok-o-no pvs. nominalizing suffix indicating when, after which: ang-ni chi ring-jok-o, na-a ring-bo when my tea has been drunk, you drink; mi jo--jok-o-no koi bal-ing-a when frying rice it bursts into puffs. (0.218)

-jol-jol-, -jo-jol- aa. via, completely, systematically, continuously: agan-jol-jol-a speak continuously, speak at the same time; dok-jol-jol-a hit repeatedly, hit many things; kat-jol-jol-a run constantly, all the time. jol-jol, jo-jol $p p$ via, along a path, road, river, (follows nominative): chi jol-jol along the water, via the water; bring jol-jol via the forest; a--sam jol-jol along the edge, of a field, etc. (3.343)

jol-oi $n$. cloth cradle that is tied between two posts of a veranda. $<\mathrm{B}$ $(6.521)$ 
jol-u-ru adv. hanging down, like a tiger's tail, hair from the head, a long beard, shirt tails. (9.255)

jol-a, jo--ol-a vi. flow, of water; rise or fall, as when water covers roads in the rainy season, recede, of floodwater; ooze, as from the ear; run, of the nose. (9.273)

jol-kep, jol-kep-kep adv. narrow, esp. of fields, (same as ap-chon-a). $(2.215)$

jom-a vi. be sick, suffer, have fever. (8.91)

jom-a gri-a vi. improve after an illness. (8.91)

jom-bru-a vi. pretend to be sick. (8.91)

jom-cha-deng-a vi. sick on and off, intermittently, (lit. sick-stand). (8.91)

jom-ket-a vi. sick all the time. (8.91)

jom-ok-a vi. a little sick. (8.91)

jom-pek-ing-a vi. repeatedly sick. (8.91)

jom-pin-ek-a vi. sick all the time. (8.91)

jom-ren-di-a vi. mildly sick, a bit sick. (8.91)

jom•-a vi. move stealthily. jom•-e-jom•-e $a d v$. moving stealthily. (9.319)

jon-mo, jor-mo $n$. birth. $<\mathrm{B}(6.77)$

jong, jong-gip-a, ang-jong $n$. younger brother, mother's younger sister's son, father's younger brother's son, cousin-brother. (6.614)

jong-a-da $n$. brothers, younger and older. (6.62)

jong-ju-a vt. stack up, of plates, pots, glasses, baskets, etc. (9.647)

jong-sa-ri $n$. wife's younger brother, husband's younger brother. (6.614)

jong--, jong $n$. bug, worm, insect, (A•chik: jo•-ong). (4.461)

jong-chret $n$. a small insect that lives in thatch and eats paper and cloth. (4.469)

jong-dol $n$. ground-dwelling insect, larger than a grasshopper, that makes noise in evening. (4.469)

jong-kang-ku $n$. inchworm. (4.467)

jong-king $n$. immature grub of a ground dwelling insect. (4.474)

jong--men $n$. grub, larva, early stage of an imature insect, before na-di. $(4.474)$

jong--mot $n$. small bug, hardly $3 \mathrm{~mm}$. long, that eats small channels in bamboo or wood. (4.469)

jong-sip-in $n$. a large hairless leaf-eating caterpillar with colored spots. $(4.467)$

jong-su $n$. a small caterpillar with stinging hairs that causes an itch. $(4.467)$

jong-tim-brot $n$. black insect that lives in the earth and curls up, the size of a finger joint. (4.469)

jor-mo, jon-mo $n$. birth. $<\mathrm{B}(6.77)$

jor-na $n$. watering pot. $<\mathrm{B}(5.436)$ 
jot-a vt. insert, poke into, with a finger, a stick, etc., as a feather into a turban; put on, wear, as a hair clip, a feather in a turban; insert a stick through leaves to hold them together; less forceful than dat-a, less pointed or cutting than su·-a. -jot-, -jot-jot-, -jrot- $a a$. insert, be tight; squeezed in the middle: sik-jot-a, sik-jrot-a plant in between other plants; song-jot-a replace a post; jang-jot-jot-a be squeezed, narrower in the middle than at the ends; sal-jot-jot-a pull hard, as when tying string; rim•-pi-jot-a, rim•-pi-jrot-a grab in the middle, squeeze in the middle. (9.571)

jot-chrak-a $v t$. remove weeds by cutting underground with a se-ni (flatbladed digging tool). (5.156)

jot-chring-chri-a $v t$. poke into something and lift it up, dirt with a shovel, straw in order to toss with a ka-rail (tossing tool). (9.571)

jot-dik-a $v t$. poke someone to call attention. (9.571)

jot-drak-a vt. poke and tear off, of paper, cloth. (9.571)

jot-du-a $v t$. echo in bo-du-a jot-du-a report bad things about someone, (lit. pierce upward). (7.25)

jot-ge-gel-a vt. poke back and forth clumsily, as children might do it, as with a stick in a hole or in the branches of a tree, wood into a fire. (9.571)

jot-ket-a vt. insert, plug up. (9.571)

jot-prot-a $v t$. poke through. (9.571)

jot-ru-ru-a $v t$. poke into, as poke with a stick into a tree or a nest. (9.571)

jot-tap-a $v t$. insert, as thatch to repair a hole in the roof. (9.571)

jra-o-jra-o $a d v$. flaming up, burning vigorously, brightly. (5.425)

jrak-jrak $a d v$. lots, in flocks, esp. for chicks, sometimes for children. $(3.342)$

jrang-a vi. be light, in contrast to darkness; become light, of twilight, moonlight, when clouds clear up, when lamps are turned on; burn up strongly, of fire, lamps. (3.554)

-jret- $a a$. tightly: ka-jret-a tie tightly; sal-jret-a pull tightly; ga--jret-a step on hard; sep-jret-a wring out water; pa-jret-a press on medicinal herbs. (3.72)

-jring dns. every: sal-a-jring every day; sop-ta-jring every week. $a a$. often, repeatedly, always, constantly, regularly: cha-jring-a always eat; i-ang-jring-a go all the time. (2.71)

jring-jring, jring-na $a d v$. always, repeatedly, constantly, daily. (2.71) jring-jrot $a d v$. repeatedly, daily. (2.71)

jrip-a vi. silent, quiet, of people, animals, machines. jrip-jrip adv. silently, quietly, secretly. (3.626)

jrip-jrang $a d v$. soundlessly, silently. (3.626)

jro-a, jru-a vi. spicy, chili-hot, burn from taste or touch of chilies. (3.682)

jro-a, jru-a vt. swim, of people, fish, etc. (9.279) 
jro-bat-a $v t$. swim across. (9.279)

jro-jro dak-a $a d v$. a little chili-hot. (3.682)

jrong-a vi. die down, of plants, almost dead. (4.696)

-jrot-, -jot-, -jot-jot- aa. insert, be tight; squeezed in the middle: sik-jot-a, sik-jrot-a plant in between other plants; song-jot-a replace a post; jang-jot-jot-a be squeezed, narrower in the middle than at the ends; sal-jot-jot-a pull hard, as when tying string; rim·-pi-jot-a, rim--pi-jrot-a grab in the middle, squeeze in the middle. (9.571)

ju-al $n$. yoke for cattle. $<\mathrm{B}(5.835)$

ju-gai-a vt. supply, support, in return for payment or when supporting a child or an indigent person. $<\mathrm{B}(8.446)$

ju-mang, ja-ju-mang $n$. dream. ju-mang nik-a $v p h$. have a dream, (lit. see a dream). ju-mang-cha nik-a $v p h$. have a dream, (lit. see in a dream). ju-mang-cha a-gan-a $v p h$. speak by a dream. (7.96)

ju-a, ju-grik-a vi. have sexual relations. (6.72)

jung-ki-ba-ring $n$. a thorny plant, a small kind of brinjal or eggplant. (5.23)

jur $n$. force: jur dak-e forcefully, insistently. $<\mathrm{B}(7.56)$

jut-ta $n$. rubber shoes. $<\mathrm{B}(6.523)$

ka-a vt. tie, tie with string, rope, or by twisting with flexible bamboo strips; put on, wear, of a turban; build by tying, of a fence, a barrier in a stream; make friends. (9.65)

ka-bai-bai adv. still tied. (9.65)

ka-bak $n$. breast of a bird, front of the body. (4.471)

ka-bang-a vi. tied strongly, of a fence; often negative: ka-bang-ja weakly tied. (9.65)

ka-bik, kin-i ka-bik $n$. string for tying hair in a knot. (6.561)

ka-cha-ra int. bravo!, shouted while dancing with a head hunting sword. $(0.64)$

ka-chap-a $v t$. tie to, tie on to, attach by tying. (9.65)

ka-chim-ot-a $v t$. tie into a bundle. (9.65)

ka-da $n$. a land measure of varying size, but in Modhupur 4.8 acres, which is 16 pa-ki. $<\mathrm{B}(5.12)$

ka-di $n$. turban, the turban worn by a priest. (6.522)

ka-dik-a $v t$. echo in sik-dik-a ka-dik-a catch and tie up a thief. (9.65)

ka-ding-de-a $v t$. hang by tying with a string or rope, suspend. (9.253)

ka-e sal-a vph. pull by a rope, of a dog, cow, etc. (9.511)

ka-gep-a vt. tie together, as a fence. (9.65)

ka-go-ji, ka-gu-ji $n$. a small variety of lime. $<$ B (5.22)

ka-gop-a $v t$. tie in a bundle, as thatch; tie in a cloth. (9.65)

ka-gos $n$. paper. $<\mathrm{B}(7.28)$

ka-gos-pul $n$. bougainvillea. $<\mathrm{B}(5.28)$ 
ka-grang-a $v t$. tie together with large open spaces, of a fence. (9.65)

ka-ji $n$. a preparation of meat bits, eaten on special occasions, generally with rice beer. (5.401)

ka-jit-a vt. move something that is tied, esp. an animal. (9.511)

ka-jot-jot-a $v t$. tie tightly. (9.65)

ka-jret-a $v t$. tie tightly. (9.65)

ka-keng-krot-a $v t$. tie tightly. (9.65)

ka-kil-ang $a d v$. having no hair; bare, ground without grass. (6.26)

ka-ku-bi-dil, ka-ku-bi-du $n$. wild pencil-sized vine with indented heart shaped leaves that are $10-20 \mathrm{~cm}$. long and edible. (4.655)

ka-li $n$. ink. $<$ B (7.28)

ka-mal $n$. priest of the traditional religion. (8.51)

ka-mal, ka-mar, sil-ka-mal $n$. blacksmith. $<\mathrm{B}(8.41)$

ka-mil-a $n$. worker, laborer. $<\mathrm{B}(8.41)$

ka-mil-eng-ga $n$. tree with five lobed sour green edible fruit, liked by pregnant women. $<$ B $(4.632)$

-ka-na, -kan pvs. third person imperative, let him, let her, let them: bi-song dong-ka-na let them stay. (0.215)

ka-ni $n$. barb of a fish hook; point, corner, such as a corner of a rice field, of a piece of paper; bottom corner of a basket. $<\mathrm{B}(5.843)$

ka-rail $n$. a tool consisting of a long pole with a spike on the end, used for tossing straw. (5.835)

ka-rak-a vt. tie tightly, strongly: am·-pang ka-rak-ja-o-de ru-rong-a if thatch is not tied strongly it tumbles down. (9.65)

ka-rang $n$. dirt on the body; filth. (6.58)

ka-ri $n$. soda that is used in cooking, and that is either purchased or made by filtering water through ashes. (5.405)

ka-ri, ka-ri-o $l w$. next year. (2.66)

ka-ri chang-ha-o nph. year after next. (2.66)

ka-ri ka-rang-o $n p h$. next year. (2.66)

ka-ri-chi $n$. liquid soda solution, used in cooking. (5.405)

ka-ri-sim $n$. salt, (variant of kai-sim). (5.405)

ka-rik-rik-a vt. retie, as loose thatch; tie again and again. (9.65)

ka-rip-rip-a, ka-tip-tip-a $v t$. tie lots and well. ka-rip-rip $a d v$. (9.65)

ka-rit-chu-a vt. tie together, as string. (9.65)

ka-rok $n$. skin disease, of animals or people, eczema. (8.953)

ka-ru-ru-a $v t$. tie in many places, repeatedly. (9.65)

ka-sa $n$. kind of metal, heavier than ordinary aluminum of pots, sometimes used for plates, drinking cups, and gongs. $<$ B (5.88)

ka-si, ka-si bol, ka-si-jak-srem n. a medium sized thorny tree with small, somewhat willow-like leaves, lots of little round fruit eaten by birds, and good heartwood for posts, (Beng. kui-ra-ka-ta). (4.632) 
ka-sim, ka-ri-sim, kai-sim, sim $n$. salt. (5.405)

ka-srok-a $v t$. tie, loosely, tie with a knot that is easily untied, like a slipknot. (9.65)

ka-steng-a $v t$. tie with a knot that is not easily untied, not a slipknot. (9.65)

ka-stik-a vt. tie tightly. (9.65)

ka-stong-a $v t$. a way of tying together two crossing roof pieces so that the visible parts of the tie strips cross each other; contrasts with a-mak ki'-pret-a where the visible parts of the tie strip run parallel. (9.65)

ka-ta ba-ju $n$. upper arm rings. (6.564)

ka-ti, gol--ka-ti $n$. a small prepared stick used to beat gongs; match stick. $<\mathrm{B}(8.85)$

ka-tip-a $v t$. cover by tying, as a banana leaf is tied over a rice beer pot, as eyes are blindfolded with a cloth; wind with string; wrap around. (9.62)

ka-tip-tip-a vt. tie thoroughly. (9.65)

ka-tom-a $v t$. tie up in bundle; tie in a cloth; pack. (9.65)

ka-wak $n$. an itching skin disease. (8.953)

ka'-a vi. bitter. (3.682)

ka'-a vt. do, work, (A•chik, A•pal). (8.433)

ka-bak $n$. front of the body; breast of a bird. (6.245)

ka-brap-a vi. taste of too much salt or soda. (3.682)

ka'-bre-o-a vi. mixed bitter and bre-o-a taste. (3.682)

ka'-cha-a vt. speak angrily, scold angrily, bawl out, insult. (7.36)

ka'-chi-a vi. taste that combines bitter and sweet. (3.682)

ka'-ding-a vi. laugh, smile. (7.29)

ka-ding-gek-gek-a, ka-ding-gak-gak-a vi. laugh heartily, constantly.

ka'-ding-git-chi-a $v t$. laugh insultingly. (7.27)

ka'-ding-kak-ka-ri-a, ka-ding-kang-ka-ri-a vi. laugh largely and loudly. (7.29)

ka-ding-rek-rek-a vi. laugh very loudly. (7.29)

ka-ding-sim-ik-a vi. smile. (9.44)

ka-ding-wak-wak-a vi. laugh a lot. (7.29)

ka'-dong-a vt. hope. (7.725)

ka'-kim-a vi. a bit bitter, not very bitter. ka-kim-ka-kim $a d v$. (3.682)

ka-kit-a vi. itch. (3.64)

ka-kit-chu-a vi. feel tickled; have buzzing in the ear. (3.64)

ka-kol $n$. depression in the chest over the lower breast bone. (6.245)

ka'-ku-ak-a vi. very bitter. (3.682)

ka'-ma, ka'-ma-o, ka'-ma-cha $p p$. below, under; down river, (follows genitive -ni). (1.11)

ka’-ma-ni ku'-chil $n$. lower lip. (6.22)

ka'-ma-ni wa $n$. lower teeth. (6.22) 
ka-ma-ni wen-a $v t$. weaving the sides of a basket after the bottom is woven. (5.856)

ka·-mang-ka•-mang, ka·-mrang-ka•-mrang adv. good smelling. (3.688)

ka'-me-seng vi. taste that combines bitter and sour. (3.682)

ka·-o-nang-a $v t$. angry at. (7.766)

ka-o-nang-dik-a vt. angry at. (7.766)

ka•ra-o, ka•-ra-u $n$. a bad smelling cockroach that is larger than an ordinary sel-uk cockroach, and that lives in people's houses and eats things. (4.469)

ka•rek $n$. beans with a long narrow edible pod. (5.23)

ka'-rek gi-sim $n$. a long kind of black bean. (5.23)

ka-rek gip-bok $n$. a long kind of white or ash-colored bean. (5.23)

ka·-rong-ti $n$. heart. (6.27)

ka-sa-a vt. love, as one feels toward one's children, sympathize with, miss. (7.722)

ka·-sa-chak-a vt. sympathize with, pity. (7.741)

ka'-sa-pa-e int. please, a somewhat artificial translation from English. (0.64)

ka'-si-rok ka'-si-rok $a d v$. beautiful sounding, of some music. (8.83)

ka-si-rok-e grap-a vph. cry quietly, wail softly, as when someone dies, a high, quiet whine. ka-si-rok-rok $a d v .(7.29)$

ka·-sim-ang-ka-sim-ang $a d v$. beautiful sounding, touching, of speaking, as one might speak to evoke pity, of music that makes one sad or thoughtful. (3.624)

ka-sin-a vi. slow, quiet, calm, slow down; cold to the touch; cool down after a fever. (2.724)

ka-sin-chip-chip $a d v$. very softly, quietly; very cold, of things, people. (3.626)

ka-sin-chok-chok $a d v$. very slowly, softly, quietly. (2.724)

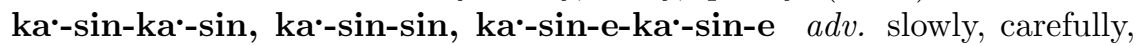
quietly. (2.724)

ka'-sin-min'-min-a vi. very slowly, quietly. (2.724)

ka'-so-a, ka'-su-a vi. noisy, unpleasantly loud, disturbing. (3.628)

ka-so-chip-a vi. unpleasantly loud, of dogs, children, airplanes, etc. (3.628)

ka'-so-ka'-rek $a d v$. noisy, of children. (3.628)

ka·-sop $n$. lungs. (6.27)

ka'-sro-ka'-sro dak-a $a d v$. unable to eat much due to feeling in throat, discomfort in the chest after eating. (8.93)

ka'-srok-e, ka'-srok-ka'-srok $a d v$. speaking beautifully, softly, as when telling story. (7.15)

ka•teng-gil-a vi. very bitter. (3.682) 
ka-teng-kil-ok-a vi. very bitter, too bitter to eat. (3.682)

ka-ting-ting $a d v$. well off, with plenty of money, property, food; without worries. (8.441)

ka'-tong $n$. heart. (6.27)

ka-wak-a vi. too strong in taste, too much sugar, salt, etc. (3.684)

kai-jol, ke-jur $n$. a palm tree that is tapped for its sap. $<$ B (5.22)

kai-sim, ka-ri-sim, ka-sim, sim $n$. salt. (5.405)

kak-ket $a d v$. true, correct. (3.44)

kak-si n. spouted earthenware pot. (5.432)

-kal- aa. more: nam-kal-a better; chu-kal-a sleep more. (3.342)

kal-bong vt. someone who eats anything, glutton; place for rubbish, garbage pit. (5.472)

kal-i-ji-ra $n$. a small variety of rice that has black husk and a good smell, grown as an a-mon crop. $<\mathrm{B}(5.25)$

kal-nim-a vi. not eat due to anger or loneliness. (7.766)

kal-si-a, kal-si-kal-tong $a d v$. dirty of the body, needing a bath; dirty, of cloth that needs a wash. (6.58)

kal-si-kal-tok, kal-ti-kal-tok, kal-ok-kal-tok $a d v$. soiled, dirty, spotted, of people, cloth, etc. (3.716)

kal-ting-a vi. rotten and smelly, stinky, as of half bad meat; rancid. (3.688)

kal-tok-tok $a d v$. dirty, soiled. (3.716)

kal-u-a, am•-pang kal-u-a vi. old thatch, good only as mulch. (5.632)

$\mathrm{kal}^{\cdot-a} v t$. play games, cards, sports. (8.81)

kal-ak-a vt. joke, play with; fool around, bother, of young people who do not obey parents, of ghosts who frighten people, of men with women. (7.26)

kal-dek-kal-tek $a d v$. small, esp. of animals. (2.215)

kal--mik $n$. a small variety of bee that makes very good honey. (4.463)

kal-tang-a vi. short, too short to fit, of sleeves, pants, pen cover, etc. $(2.215)$

kam, gam, gam-u $n$. work, labor. $<\mathrm{B}(8.433)$

-kam- aa. completely, thoroughly: ron-kam-a give everything; wakam-a rain continuously; rim-kam-a keep working, not rest; bam-kama bent thoroughly over; dong-kam-a live, dwell, stay for a long time. $(3.342)$

kam-a vi. burn, blaze. (5.424)

kam-bi $n$. top of head; flower bud, leaf bud, end of a growing vine; end, tip, extreme point; top of a tree. (4.676)

kam-du-a vi. echo in ding-du-a kam-du-a, hot, feverish. (8.93)

kam-gil-gil-a vi. burn on the surface only. (5.424)

kam-kring-krang-a vi. incompletely burned. (5.424) 
kam-ret-a vi. burn partly. (5.424)

kam•-chok-kam•-chok $a d v$. good, of well-cooked food, of rice beer. (5.46) kam•-dak $n$. real kin, immediate kin, closely related. (6.634)

-kan, -ka-na pvs. subordinating suffix, esp. with verbs of telling or ordering: kat-kan-a bi-a hit-a he ordered (him) to run. pvs. third person imperative, let her, let him, let them: bi-a re•-ang-kan let her go, she can go. (0.216)

kan- cls. generalclassifier, residual category classifier, esp. for man-made objects that lack any other clear classifier: shoes, glasses, hats, ears, houses, doors, lights, letters, books, baskets, etc. (1.31)

kan-a vt. feed, of liquids, including breast milk. (5.478)

-kan-bo, -ka-na-bo pvs. third person imperative, equivalent to -kan or -ka-na. (0.215)

kan-chi-dik $n$. small, common house lizard. (4.441)

kan-da- cls. classifier for small bunches of bananas, about eight fruit. (1.34)

kan-di $n$. three takas. (8.447)

-kan-di- $a a$. in part, partially: cha-kan-di-a eat some, leave some; sikan-di-a die before time, part way through (normal) life; tong•-kan-di part way. (3.347)

kan-gek-a, kan-gek-gek-a vt. force someone to drink, as when serving rice beer. (5.478)

-kan-ta dns. lacking a limb or tail: jak-kan-ta lacking an arm; jak-sikan-ta missing a finger; chu-kan-ta incomplete. (8.96)

kan-chi-ri $n$. worm that lives in ground and makes knobly little dirt piles, does not bite. (4.467)

kan-chi-ri chon-gip-a $n$. a small kind of kan·-chi-ri, smaller than kan'-chi-ri-ron-da. (4.467)

kan-chi-ri ron-da $n$. long thin worm, a large variety of kan·-chi-ri, about $15 \mathrm{~cm}$ long. (4.467)

kan-dam-a vi. short, of people. (2.215)

kan·-dok-a, kan•-dik-a vi. short, of people, animals, string, sticks, posts, days, etc. short of speaking, briefly. (2.215)

kan'-dok-kan'-dok adv. into lengths. (2.312)

kan-tang $n$. short sleeved. (2.215)

kan-tap $n$. tree with $25 \mathrm{~cm}$. fuzzy indented leaves and small round inedible fruit. (4.632)

kan-tong-a vi. short. (2.215)

kang-a vi. blocked off, obstructed, as by crowded things, by people on a bus, by a tree across the road, by something stuck in the throat; difficult, of a song. (9.77)

kang-dik-kang-si $a d v$. so crowded with things or people that it is difficult to get by. (9.77) 
kang-kang-pret $n$. bush with tiny doubled leaves facing each other. (4.64)

kang-ket-a $v t$. choke on, as on a fish bone, or on water when drinking; stuck in the throat: ang-a do-greng-ko at-ta-ka kang-ket-a I suddenly choked on a chicken bone. (5.479)

kang-kil-ak-a $n$. small kind of turtle with hard shell. (4.445)

kang-kon-te-a vt. pull or grab by a handle, hook, or loop, as by an umbrella handle. (9.531)

kang-a vt. support, as to prop up a banana plant with a pole. (9.58)

kang-ka-ri $n$. lower back, small of the back, (A·pal). (6.243)

kang•-ka-ri $n$. small tree with $2 \mathrm{~cm}$. round leaf, edible sour, puckering, or slightly sweet red fruit when ripe, (A•pal), (same as te'-seng-ki). (4.632)

kang-kip-a, kang-kip-kang-kip vi. adv. bend forward at the hips, as when dancing. (9.348)

kang-krang-a vi. not touching, off the ground, as a tree that has fallen only partway; squat with buttocks well off the ground; sit with one's back away from a wall. (2.11)

kang-krang-krang, kang-krang-chi-krang, kang--krang-e at-chonga $a d v$. squat with buttocks well off the ground. (9.345)

kap $n$. cup. $<$ E (5.434)

kap-pa gro, ku-pa gro $n$. legal case, a dispute that becomes subject to formal arbitration. (8.13)

kap-pi $n$. lower back, small of the back. (6.243)

kar-pu n. a kind of fish, carp. $<$ B (4.454)

Kar-tiik, Ka-tiik, Ka-ti $n$. a Bengali month that overlaps October and November. $<$ B $(2.827)$

kas $n$. ordinary, unrefined; daily language; a form of language regarded as poor or improper. $<\mathrm{B}(7.11)$

kas-tel $n$. mustard oil, used in cooking. $<$ B (5.405)

kat $n$. an itching skin disease. (8.953)

kat cha-a-ni sam nph. leaves of a species of small tree or large bush that are ground with water to rub on the skin to treat the skin disease called kat. (8.99)

kat-a vi. run, run away. (9.316)

kat-cha-a vi. ashamed, (A•chik). (7.745)

kat-chi $n$. sickle, rice cutting knife. (5.832)

kat-chi $n$. echo in mit-e kat-chi spirits. (8.54)

kat-dil-a, gat-dil-a $n$. the vertical posts near the front of a rice pounder that guide its fall. (5.87)

kat-dim-dim-a vi. run a lot, this way and that, as children do. (9.316)

kat-ding-dang-a vi. run off in various directions, to different places, run here and there. (9.316) 
kat-e chak-a $v p h$. run away and hide, as when a young man tries to avoid marriage. (6.651)

kat-gil-gil-a vi. run around and around, back and forth, as children. (9.316)

kat-gring-grang-a vi. run off in all directions. (9.316)

kat-jem-jem-a vi. run repeatedly. (9.316)

kat-jol-jol-a vi. run constantly, all the time. (9.316)

kat-jring-a vi. run repeatedly. (9.316)

kat-mik-brang-a vi. run off in fear. (9.316)

kat-mrak-a vi. run through a field and destroy crops, as animals may do. (9.316)

kat-nap-a vi. run and enter, rush in; run in and hide, take shelter. (9.316)

kat-pek-a vi. running repeatedly. (9.316)

kat-pen-sil, kat-kol-om $n$. pencil. <B (7.28)

kat-ru-rat-a, kat-ru-ra·-a vi. run back and forth. (9.316)

kat-ru-ru-a vi. run fairly fast. (9.316)

kat-sam $n$. a bush about a meter tall, with an odd yellow flower on top and long pods with an x-like cross section, the leaves of which are ground with water to rub on skin to relieve kat, an itching skin disease. (8.99)

kat-sek-a vi. run a race. (9.316)

kat-sit-tap-a vi. run away. (9.316)

kat-srap-a vi. run and catch up with. (9.316)

kat-tong-tang-a vi. run straight, directly. (9.316)

kat-tu-a $n$. an edible turtle, smaller than, and a different species from, chi-jong, (same as chep-a). <B (4.445)

kat-wen-wen-a vi. run around, all over the place. (9.316)

kat-wil-wil-a vi. run in circles, run all over the place. (9.316)

ke-a, ki-a vt. butt with horns. (4.482)

ke-bit-a, ki-bit-a vt. bring along, of things that hang, of something wrapped in a cloth or tucked into something: O-ses ri-spil ki-bit-inga Oses comes with his hanging testicles. (9.532)

ke-ching-chri-a $v t$. poke with horns: ma·-su grong-cha ke-ching-chriing-a the cow pokes with her horns. (4.487)

ke-grik-a vt. push or fight each other with horns, as buffalo. (4.482)

ke-ji- cls. classifier for kilograms. <E (1.36)

ke-jur, kai-jol $n$. a palm tree that is tapped for its sap. $<$ B (5.22)

ke-ma ka·-bo, ke-ma ka•-pa-bo int. a somewhat artificial translation of English 'excuse me'. (0.64)

ke-ma ron-a $v t$. forgive, give forgiveness. (7.39)

ke-o pro. anyone, someone; ke-o-ba with negative means nobody: ke-oba sok-ba-ku-ja nobody has arrived yet. $<\mathrm{B}(1.64)$

ke-ri-ar $n$. carrier of a bicycle. $<\mathrm{E}(5.872)$ 
ke-ring bol $n$. small tree with three-part leaves and large inedible fruit. (4.632)

ke-rok-a, ki-rok-a $v t$. shake a winnowing basket back and forth to separate the rice from the husks. (5.451)

ke-sa-ri $n$. a kind of dahl. $<\mathrm{B}(5.23)$

ke-si, ke-chi $n$. scissors. $<\mathrm{B}(5.832)$

ke'-a, ki·-a vt. permitted to marry, belonging to correct kinship groups; appropriate, as appropriate to eat with right hand: ang-a Ma-rak-ko dong-na ki--ja it is inappropriate for me to marry a Marak. (6.657)

ke-em-a n. dung; excrement. vi. defecate. (6.29)

ke-em-bu-a vi. defecate in clothes, or on a mat, etc. as a baby might; deficate a lot. (9.49)

ke-em-chi-rot-a vi. defecate just a little. (9.49)

kel, ker $n$. rice straw after grain has been removed. $<$ B (4.66)

kel-ki $n$. small wooden cotton gin. (5.835)

kel-ok-kel-ok $a d v$. coughing repeatedly. (9.45)

kel-wi $n$. tree with rather dusty yellowish bark, thin $15 \mathrm{~cm}$. seed pods and smallish $2 \mathrm{~cm}$. leaflets on $20 \mathrm{~cm}$. leaf, (Beng. ko-roi). (4.632)

ken-a vi. be afraid. (7.764)

ken-a gri $p p p$. without fear, fearless. (7.746)

ken-chak-a vi. afraid for someone else. (7.764)

ken-dil-a vt. frighten, cause someone to be afraid. (7.764)

ken-ja-brang-a vi. terrified, helpless with fear. (7.764)

ken-ja-gok-a vi. scream with fear. (7.764)

ken-kok n. coward. (7.764)

ken-sik-a $v i$. nervous, worried, a little scared. ken-sik $a d v$. (7.764)

ken-ta-a vi. raggedy, shabby, of cloth, old houses, pots, jokingly of people. (9.292)

ken-ti-ri $n$. very small-leafed flowering tree, with sour fruit liked by pregnant women, (Beng. te-tul). (4.632)

ken'-a vt. brush, comb hair. (6.57)

ken-chrak-a vt. comb vigorously, as when itching, or to get rid of lice. $(6.57)$

ken·-dok-a vi. short, of sticks, shirts, etc. (2.215)

ken'-srok-a vt. comb gently. (6.57)

keng-krang-a vi. thin, skinny, of animals. keng-krang-krang, keng-krang-si-si $a d v$. (6.46)

kep-a vt. hold or pinch, as with tongs; pinch, of a crab. -kep- $a a$. hold, grab, squeeze: jak-kep-a hold pinched in a nearly-closed fist; pak-sikkep-a carry under the arm; ja-kep-a hold pinched between one's feet; dang-kep tongs. (9.531)

kep-kep-a vi. tremble with fright, especially of the lips and mouth. $(9.322)$ 
kep-kep-dang-dang $a d v$. talking constantly, repeatedly. (7.12)

kes-ki wa $n$. medium sized variety of bamboo that is good for basket making. $<$ B (4.637)

-ket- $a a$. filled, squeezed tight, stuck: gap-ket-a filled up tight, no more space, squeezed in; dap-ket-a push wet mud into cracks, bury; jot-ket-a insert, plug up; chang-ket-a stuck, crowded, choke; nap-ket-a enter and stay. (2.22)

ket-a vt. close with hook or latch, but not with a lock. (9.61)

ket-chi-e $a d v$. on the side. ket-chi-e chu-a, ket-chi-chu-a $v p h$. sleep on one's side. (9.349)

ket-chi-grik-a vi. side by side. (2.25)

ket-chi-o, ket-chi-cha $p p$. at the side of, beside, on the side, (follows genitive -ni). -ket-chi- $a a$. to the side, crosswise: chit-ket-chi-a cut bamboo strips crosswise to the inner and outer edges; ni-ket-chi-a look to the side; pit-ket-chi-a cut off with a cut perpendicular to the surface. (1.11)

ket-chi-ra, ket-chra, ki-chra $n$. a red ant that appears in large swarms and that has a painful but non-poisonous bite. It makes nests in folded leaves. (4.465)

ket-chi-ra bi-top, ket-chi-ra bi-tip $n$. nest of leaves made by red ants, with jong·-men (grubs) inside. (4.475)

ket-ta $n$. a blanket made by tying layers of thin cloth together. $<\mathrm{B}$ (6.591)

ki ja-ni? int. who knows? <B (0.66)

ki-a, ke-a vt. hang, (see ke-a for compounds). (9.532)

ki-a, ke-a vt. butt with horns, (see ke-a for compounds). (4.482)

ki-rong $n$. echo in $\mathbf{a}^{\cdot-t o t}$ ki-rong, property of a single individual, as opposed to family property. (8.423)

ki-rot-ki-rot, ki-rot-bak-rot $a d v$. a bit here, a bit there, as in giving things in bits rather than all at once. (3.347)

-ki- - dns. dirt, especially body dirt, dung: ging-ki nose dirt, snot; mik-ki eye dirt; wa-ki tooth dirt, tartar; wal-ki coals from a fire; ki-bit-bit dung beetle: su-dap-ki-ki dirty, unwashed. (6.29)

ki·-a vi. defecate, (A•chik). (9.49)

ki·-a, ke•-a vt. permitted to marry, belonging to the correct kinship groups; appropriate, as appropriate to eat with the right hand. (6.657)

ki-bit-bit $n$. dung beetle. (4.469)

ki-bong-bet-a vi. defecate vigorously, have diarrhea. (9.49)

ki-brek-a vi. have diarrhea. (9.49)

ki-chrak-a vi. defecate forcefully, have diarrhea. (9.49)

ki-dam-bu-a vt. defecate plentifully or in the wrong place, as children do. $(9.49)$ 
ki-dik $n$. latrine. (5.622)

ki-gol $n$. leaves or a piece of bamboo used to clean excrement. (6.57)

ki·-jil-jil-a vi. defecate a lot. (9.49)

ki-jrot-a $v t$. defecate a little in the wrong place as by a child. (9.49)

ki-ka-rok $n$. bits of dirt in the hair, dandruff. (6.58)

$\mathbf{k i} \cdot \mathbf{- m e}$, ki-mi $n$. tail; the projecting upper part of the top of a house post cut to fit a cross piece. (4.473)

ki-pak-pak-a vi. defecate a lot. (9.49)

ki-pat $n$. cross pieces, strengthening diagonals, of a basket bottom. (5.854)

ki--pil-a $n$. the part of a traditional gan-do (loincloth) that goes between the legs and tucks in at the waist in back. (6.527)

ki-pret-a, a-mak ki--pret ka-a vt. a way of tying together two crossing roof pieces, so that the visible parts of the tie strips run parallel, contrasting with ka-stong-a where the visible parts cross. (9.65)

ki-pring $n$. morning defecation. (9.49)

ki-rong-a, ki'-rong-rong-a vi. diarrhea. (9.49)

ki-ru-ru-a vi. defecate plentifully. (9.49)

ki-sam-bil-ak $n$. disease when something like skin comes out of the anus. (8.97)

ki-sam-bu $n$. anus; back end of an object, filter of a cigarette, back end of an inch worm; bottom of a pot. (6.243)

ki-sang $n$. buttocks, backside; bottom or back end of an object, as a flashlight, basket, glass, pot. last, previous, most recent: ja ki-sang last month. (6.243)

ki--sang song-a $v p h$. sit down (rude, lit. post one's butt). (9.345)

ki-sang tik-a vph. with one's back toward, turn one's back, (lit. with the buttocks toward). (9.349)

ki'-sang-cha-na $a d v$. backwards. (2.14)

ki-sang-chak-a vi. follow behind; fall behind, cannot keep up. (9.328)

ki-sang-ki-sang $a d v$. one following the other, single file. (2.14)

ki-sang-o, ki-sang-cha $p p$. behind, in back of, (follows genitive -ni), (lit. at the buttocks of). (1.11)

ki-sang-tap $a d v$. less than half filled, of a basket, etc., with only a little in the bottom. (3.348)

ki-sang-ti $n$. hind end of an animal; buttock. (4.473)

ki'-si-a vi. fart. (9.49)

ki-si-sru-a vi. fart a lot. (9.49)

ki-sik $n$. bottom corner of a basket. (5.854)

ki-sing-kop $n$. lowest area of the back, area at the back of the pelvis. (6.243)

ki-song-a vi. echo in at-chong-a ki--song-a sit down (rude). (9.345) 
ki-sru-sru-a vi. have diarrhea, lots of defecation. (9.49)

ki-tap $n$. diaper, baby napkin. (6.521)

ki-ting, ki-sang-ti ki-ting $n$. hard point at lower middle back of the pelvis, coccyx. (6.243)

ki-wa $n$. leaves or a small piece of bamboo used to clean excrement. ki-wi-a $v t$. clean excrement with a stick or leaves. (6.57)

kiir-ton $n$. a type of circle dance. $<\mathrm{B}(8.83)$

kil n. cotton. (5.29)

kil-a, ha-ba kil-a vt. stage of clearing after burning a field, when unburned or partially burned bits that remain are gathered into piles for further burning. (5.113)

-kil-ak- $a a$. open: sal-kil-ak-a pull open; o-kil-ak-a open, as a box, a covered pot, door, trunk, book, skin of a banana, jackfruit; king-kil-ak-a open, of flat things, books; pull up, of pant legs, lunggi, skirt. (9.60)

kil-ap $n$. cotton fluff after the seed has been ginned out; the cottony part of a bol-chu tree. (4.676)

kil-as n. class. $<\mathrm{E}(8.6)$

kil-chi-ri $n$. a good variety of cotton. (5.29)

kil-ding $n$. fine string, thread, including jute thread. (6.594)

kil-e-rep-kil-e-rep $a d v$. small bits of flat things such as bits of paper or cloth. (2.215)

kil-gon, kil-gron $n$. cotton seed. (4.677)

kil-te, kil-ti $n$. cotton ball before ripening, before it has burst. (4.677)

kil-teng $n$. ordinary variety of cotton. (5.29)

kim-a $n$. memorial post that is set up to honor a dead person. (8.58)

kim-a vi. to make a noise, as thunder; make a sound. (4.342)

kim-a vt. marry, get married: me-chik me-a-sa-ko kim-a the woman marries the man. (6.651)

kim-at kim-chi $n$. the relation of kin groups formed by marriage, the relation of an individual to his or her spouse's kin group: ang-a Re--mani kim-at kim-chi I am married to the Re'-ma lineage. (6.651)

-kim-bak- $a a$. shortened, abbreviated, partly, halfway: den-kim-bak-a cut a little, partly; cha-kim-bak-a eat a bit, not finish; wal-kim-bak-a half the night; rat-kim-bak-a cut partway through. (2.215)

kim-bal-o-bi-du $n$. a thorny vine, thick as a thumb with $30 \mathrm{~cm}$. leaves with parallel veins. (4.655)

kim-bing-a vi. to thunder in the distance. (4.342)

kim-brok-a vt. put one's hand in and grab, as from a hole or pot. (9.531)

kim-cha-a vi. to thunder. (4.342)

kim-chap-a $v t$. add a second wife. (6.652)

kim-il $n$. body hair, fur of an animal, small feathers, down. (6.25)

kim-il-a, chi kim-il-a vi. dirty, cloudy, of liquids. (3.716) 
kim-il-ra-ra $a d v$. hairy. (6.25)

kim-it-a, sik-kim-it-a vt. extinguish, put out, as a fire or match; turn off, hurricane lamp, flashlight. (5.424)

kim-pret-a vi. thunder, powerful thunder clap. (4.342)

kim-ru-ru-a vi. long lasting thunder, thunder which sounds as if it moves across the sky. vi. echo in kim-pret-a kim-ru-ru-a thunder. (4.342)

kim-sot-a $v t$. end a $\mathbf{a}^{\cdot}$-kim relationship that a marriage forms between two lineages. (6.655)

kim--bik $n$. peak of a roof, ridge, the part of the roof above the ridge-pole where the thatch is bent over it. (5.632)

kim--bik pi-a $v p h$. 'break' the thatch to bend it over the ridge of the roof. (5.632)

kim--bik-ni krong $n p h$. post at one end of a house that supports the ridgepole. (5.633)

kin-a-a vt. hear, listen. (3.622)

kin-a-chak-a $v t$. agree, follow what one hears. (7.33)

kin-a-dik-a vi. ugly in sound, opposite of kin-a-to-a beautiful to hear. (3.624)

kin-a-rik-a $v t$. listen to something passing or moving: nang-ni a-gana-ko ang-a kin-a-rik-e chok-ing-a I am listening and writing your speech. (3.622)

kin-a-si-a vi. sound bad, sound ugly, (same as kin-a-dik-a). (3.624)

kin-a-to-a vi. beautiful to hear, nice sounding. (3.624)

-kin-ap- $a a$. comfortable, pleasant: rim-kin-ap-a pleasant to touch, soft; dong-kin-ap-a in a good state, comfortable; chu-kin-ap-a sleep well, comfortably; pin-kin-ap-a spread comfortably, as a blanket; chukin-ap-a comfortable for sleeping. (3.455)

kin-i $n$. hair of the head. (6.25)

kin-i-ki-chrot $n$. tuft of hair, hair bit at middle back of head, just above neck, the Hindu hair tuft. (6.25)

kin-ok-a vt. put one's hand into a hole and grab, grope. (9.531)

kin-tu conj. but, however. $<\mathrm{B}(1.4)$

king- cls. classifier for flat things, ears, leaves and esp. for paper and things made from paper, including books, but not cloth. (1.32)

king-chon-a, king-chon-king-chon vi. small of flat things, of mats, winnowing baskets, leaves, paper, books. (2.211)

king-dal-a vi. big of flat things, of paper, banana leaves, boards, ears, bread, paper, books. (2.211)

king-gal-a $v t$. uncover, take off cloth, cover of a pot, etc., turn right side up, as a boat. (9.62)

king-kil-ak-a, king-krak-a vt. pull up, pant legs, lunggi, skirt; open, of flat things, books. (6.53) 
king-king $p p$. until, up to, (follows dative -na): sal-ja-chi-o-na kingking until noon. (1.12)

king-kip-a vt. turn over, turn upside down, of baskets, pots, small objects; capsize a boat; especially to turn over a basket, plate, etc. in order to enclose something under it; put on cover, of a pot. (9.62)

king-kip-dap-a $v t$. place a plate, basket, etc. upside down over another thing in order to cover it. (9.62)

king-ma, king-ma-ma, king-ma-king-ma adv. large of flat things, paper, cloth, leaves, winnowing basket, mat, etc.: king-ma-a vi. (2.211)

king-ring-king-ring adv. digging, of plowing, hoeing, of a pig rooting. (9.576)

king-tal-a $v t$. open up, of books, of flat things previously folded, of clothes to dry. (9.60)

-kip- aa. upside down, closed up in: sin-kip-a turned upside down; king-kip-a turned upside down over; jak-kip-a hold in a closed hand. $(9.214)$

kip-a, jak kip-a $v t$. bend the fingers to press against palms; grab or hold something in the fist. (9.46)

kit-a vt. dig a hole such as a grave, tank or well; pull out of a hole, dig up. (9.576)

kit-bol-bol-a $v t$. stir up rice in a beer pot with the hand, in order to spoil it. (5.455)

kit-brok-a vt. stick one's hand in and grab. (9.531)

kit-chrak-a $v t$. scratch up dirt, of dogs. (9.576)

-ko, -ku cm. accusative case marker that marks the direct object of a clause or sentence: ang-ko me. (0.12)

ko-a, ku-a vt. do cane work, weave cane as part of basket making. (5.856)

ko-ar, ko-ra $n$. fenced in area for animals, pen. $<$ B (5.622)

ko-bi-ras, ko-bi-raj $n$. herbal doctor. $<\mathrm{B}(8.91)$

ko-bi-ta $n$. poem, poetry. $<\mathrm{B}(7.28)$

ko-chi-rok, sko ko-chi-rok $a d v$. with shaven head, bald, without hair. (6.26)

ko-dal, ku-dal $n$. big wide hoe for digging out dirt. (5.831)

ko-dam, ku-dam $n$. pillow. (6.591)

ko-dom $n$. undistinguished tree with ordinary $15 \mathrm{~cm}$. leaves and inedible fruit. $<$ B (4.632)

ko-e-ri $n$. dark brown, purple. $<\mathrm{B}(3.534)$

ko-ka $n$. turban. (6.522)

ko-ka, ku-ka $n$. a little boy, older than a gen-da but not yet a pan-te. Used in calling. $<$ B $(6.822)$

ko-ka-rok, ku-ka-rok, sku ko-ka-rok $a d v$. bare, hairless, bald; bare ground as in a courtyard between houses. (6.26) 
ko-ka-rong, ku-ka-rong adv. hairless. (6.26)

ko-ki, ku-ki $n$. a little girl, older than a gen-da but not yet a me-tra. Used in calling. $<$ B $(6.822)$

ko-ku-rang-rang $a d v$. bald. (6.26)

ko-mil-a, ku-mil-a $n$. orange tree, orange fruit. $<$ B (5.22)

ko-mil-ong-a, ku-mil-ong-a, ko-mil-ong dong-a vi. naked above the waist. (6.552)

ko-mor, ku-mor, ko-mol $n$. waist. $<\mathrm{B}(6.243)$

ko-pal $n$. forehead. $<$ B (6.22)

ko-ping $n$. head cloth, esp. one worn by a woman, may be tied under the chin. (6.522)

ko-ra $n$. a land measure, 1/100 acre, the smallest measure of land, (same as dis-mel). $<\mathrm{B}(5.12)$

ko-ra $n$. small bowl. $<$ B (5.434)

ko-ra-a, ku-ra-a vi. with congenitally twisted feet, limp. $<\mathrm{B}(8.96)$

ko-re, ku-ri n. crazy person. (8.92)

ko-ros $n$. money wages. $<\mathrm{B}(8.435)$

ko-ta $n$. words, what is said. $<\mathrm{B}(7.28)$

ko-ta $n$. can, food tin. $<\mathrm{B}(5.436)$

ko-tip, ku-tip n. man's turban; head cloth wrapped over forehead and tied in back; top of memorial post. (6.522)

ko-a, $\mathbf{k u}^{\cdot-\mathbf{a}} v t$. draw water up from an open well, dip out water. (9.277)

koi $n$. puffed rice, popcorn. $<\mathrm{B}$ (5.403)

koi-chong-a vi. coming up to a peak, as an ant hill. koi-chong-chong $a d v$. (2.312)

koi-ra, ko-rai-a $n$. round-bottomed cooking pan with two handles, wok. $<$ B (5.436)

koi-sak $n$. a fairly bland green leafy vegetable, a climbing plant with red stalks and $2 \mathrm{~cm}$. heart shaped rather succulent and edible leaves. $<\mathrm{B}$ (5.23)

-kok dns. the one who: chau-kok thief; mat-u-kok greedy person; siik-kok teacher; ken-kok coward. (6.821)

kok $n$. basket; mainly A $\cdot$ chik, but occurs in Mandi as a categorizing prefix in compounds for particular kinds of baskets. (5.852)

kok- $c p$. prefix for types of baskets: kok-si fish basket. kok $n$. basket. $(0.17)$

kok-kim-a-o $p p$. underneath, (follows genitive -ni), (A•chik). (1.11)

kok-kreng $n$. roughly made, openly woven, middle sized carrying basket for firewood, etc. (5.852)

kok-sep $n$. small basket generally used to hold small animals, birds, fish, etc. $(5.852)$

kok-si $n$. basket for holding fish or eels. (5.852) 
kol $n$. a machine, such as a pump, a rice mill, a sugar cane press. $<\mathrm{B}$ $(5.835)$

kol- $c l s$. classifier for holes such as windows, doorways, holes in the ground or in a tree. (1.32)

kol-a, kol-am-a vt. pick up small things, as from the ground, gather. $(9.535)$

kol-a-chi num. thirty, (A•chik). (3.3730)

kol-a-pul $n$. a beautiful orange flower. $<$ B (5.28)

kol-am-a vt. pick up, lift up, gather, of small things from theground; collect fallen fruit. (9.535)

kol-chang-bong-a num. one hundred, (lit. $20 \times 5$ ). (3.3799)

kol-chang-bri num. eighty, (lit. $20 \times 4$ ). (3.3780)

kol-chang-gin-i num. forty, (lit. $20 \times 2$ ). (3.3740)

kol-chang-git-tam num. sixty, (lit. $20 \times 3$ ). (3.3760)

kol-chon-a vi. small, of holes: kol-chon-chon $a d v$. (2.211)

kol-dal-a vi. big, of holes. (2.211)

kol-e-ra $n$. cholera. $<\mathrm{E}(8.92)$

kol-grik, kol num. twenty, (A•chik). (3.3720)

kol-grik-bong-a, kol-i bong-a num. twenty-five, (A•chik). (3.3725)

kol-ki $n$. pottery top of a water pipe that holds the tobacco. $<$ B (5.429)

kol-ma vi. big, of holes. (2.211)

kol-ma-ma $n$. big hole. (2.38)

kol-om $n$. pencil, pen. $<\mathrm{B}(7.28)$

kol-om, kol-om ka-ti $n$. cutting, sprout, to be used in planting. $<\mathrm{B}$ $(5.14)$

kom $n$. few, little. <B (3.345)

kom-bol $n$. blanket, woven of heavy thread. $<\mathrm{B}(6.591)$

kom-i-a vi. lessen, reduce, as to come down in price. $<$ B (3.345)

kom-pru $n$. a big kind of black stinging ant that comes out in rainy season, (same as gong-gang). (4.465)

kom•-a vi. bend, make oneself small, cower, as a dog in fear, as a person in the presence of someone powerful. (9.641)

kom•-si-a vi. crumple up small, as when sick or cold. kom·-si kom·-jom dak-a $a d v .(9.348)$

kom•-tet-a vi. curl up tightly, of a person or an animal when strongly resisting someone who is pulling. (9.641)

-kon ts. perhaps, probably: nam-ku-ing-a-kon (it) is probably still good. (0.31)

kon-jon $n$. a locust tree with flowers, small oval leaflets on compound leaves, and long skinny edible fruit that is sometimes eaten with dahl, (Beng. sa-di-na). (4.632)

kon-jon bi-jak $n$. edible leaves of the kon-jon (locust?) tree. (5.23) 
kon-ta $n$. a digging tool a with long straight wooden handle and a narrow straight blade at the end that is used for smoothing the sides of holes, digging up tubers, etc. $<$ B (5.831)

kon-a vi. coiled up in a circle or spiral, as string; curled up, as a snake, dog, cat. (9.641)

kon-dau-la $a d v$. circling, encircling, twisted around, of a road, snake, as a dog sleeps. (2.315)

kon-dok $n$. sections of a building, areas divided by partitions. kon-dokcls. classifier for sections, rooms. (5.635)

-kong- dns. scooped out, hollow: ring-kong hollow log used to feed animals; bol-kong-bang hole in a tree trunk; kong-kep area inside the curl of the edge of the ear. (2.38)

kong-a vt. dig by scooping and scraping rather than by chopping. (9.576)

kong-bang, bol-kong-bang $n$. hole in a tree trunk, hollow space at base of a tree or higher up. (4.673)

kong-brim $a d v$. of mixed colors, having several different colors, as black, white and red, of a tiger or dog. (3.538)

kong-dep-a vi. limp, lame. (8.96)

kong-kang, kong-kang-kang, kong-krang $a d v$. thin, of a person, so that bones show. (6.46)

kong-kep, na-kong-kep $n$. the area inside the curl of the edge of the ear. (6.22)

kong-kep-skep $a d v$. full, no space, too many things to fit. (2.22)

kong-si-kong-rok $a d v$. doing lots of work, work hard, busy. (8.433)

kong-tok-a, kong-brim-kong-tok-a vi. mixed colors, as in a lunggi pattern. kong-tok-kong-tok $a d v$. (3.538)

kong-krot-a vi. bent, curved, as an umbrella handle; curly, of hair; curled up, of a magazine cover, a dog's tail; curled up position in sleeping, sitting; with bent legs. (2.318)

kong-krot-e chu-a $v p h$. sleep in a curled-up position. (9.343)

kong-krot-kong•-krot adv. curl, curly, of hair. (6.561)

kop jik-se $n$. the pair of half bamboos that run the length of the roof, one below and one above the rafters and to which the thatch is tied, (lit. wife-husband pieces). (5.632)

kop ran-di $n$. a single length of half bamboo that runs the length of the roof, (lit. widow piece). (5.632)

kor-gus $n$. rabbit. $<\mathrm{B}(4.419)$

kos-mos $n$. an ornamental flower, cosmos. $<$ E (5.28)

kot-tin-a, mang-got-cha kot-tin-a vi. walk with a stick, as an old person: kot-tin-i ram-a ri-a walk with a stick. (8.96)

kra cha-a vi. modest, embarrassed, ashamed. (7.745)

kra cha'-na gu-al-a $v p h$. lacking modesty, (lit. forget to be modest). $(7.745)$ 
kra dong-ja $v p h$. show no shame. (7.745)

kra-a vi. be correct, right, suitable, appropriate, fit, competent, allowed to marry: Sang-ma-ming Ma-rak-ming dong-na kra-a for Sangma and Marak to marry each other is correct. (6.657)

kram $n$. type of drum, a bit smaller than da-ma and with one end that tapers to a small size. (8.85)

kram-chi $n$. sweat, perspiration, (A•chik gram-chi). (6.29)

kram-chi-a vi. sweat, perspire. $n$. perspiration. (9.49)

kram-ni pi-sa $n$. very small and sacred drum, (same as na-tuk), (lit. child of the kram). (8.85)

-krang- $a a$. open, with space between: ting-krang-a open, of umbrellas; kang-krang-a off the ground, as a tree that has fallen only partway; de-krang-a resting with one end on off the ground, hold a foot in the air. (2.25)

krang-a, krang-krang-a, krang-krang dak-a vi. clear up, of weather, clouds, or a mess; clear out of the mind, forget; empty, of a pot without water, etc. (4.323)

kre-o-kre-o $a d v$. with a crackling sound, of dry leaves being crushed, an egg breaking, of shrimp, of eating food that is not fully cooked. (3.624)

kreng-a vi. independent, not taking directions from others. (9.73)

kreng-chak-a vi. defend, protect. (9.73)

kreng-ding-a vi. strong, forceful. (7.56)

-krep- $a a$. crush: rim-krep-a grab and crush; dok-krep-a crush by hitting; so-krep-a cremate, burn a dead body. (9.578)

kri-mi, kri-mi jo-ong $n$. disease characterized by worms coming out from the anus. $<$ B $(8.97)$

kriim bi-skut $n$. a kind of purchased bisquet. $<\mathrm{E}(5.408)$

krim-kru $a d v$. with many people; with many children and kinsmen: angni ang-su ang-di bang-a krim-kru my grandchildren and children are many. (6.657)

kring-a vi. stretched tight, of cloth, rope, hide over a drum; stretch an arm or arms out straight toward the front or side; stretch fingers out straight. kring-kring, kring-kring dak-a $a d v$. stretched tight, of cloth or string, without wrinkles. (9.642)

-kring-krang aa. uncontrolled, incompletely, scattering around: chakring-krang-a get up quickly throwing covers around; go-kring-kranga throw around in an uncontrolled way; ru-kring-krang-a take down and scatter around, of old thatch; kam-kring-krang-a incompletely burned; pin-kring-krang-a incompletely thatched. (9.54)

krip-a vi. closed, of an umbrella, covered, of a pot, basket. (9.211)

krit-a $v t$. sacrifice to spirits, (same as a-mu-a). (8.562) 
krok-a vt. put on or wear, of something that requires insertion of a body part rather than wrapping, such as a shirt, sweater, socks, pants, etc. $(6.53)$

krok-dik-a vi. not good for wearing, as of dirty clothes, uncomfortable of clothes. (6.53)

krok-to-a vi. good for wearing, comfortable. (6.53)

krong n. post. (5.633)

kru- cls. classifier for spans from the thumb to the end of the middle finger or little finger. (1.36)

-ku- aa. still, anymore: jom-ku-ing-a still sick; sa-dik-ku-ja-ing-a not hurting yet. (0.41)

ku-a $n$. open well, dug well. $<$ B (5.642)

ku-a, ko-a $v t$. do cane work, weave cane as part of basket making. (5.856)

ku-a do-ri $n p h$. well rope. (5.835)

-ku-ak-a $a a$. in a strong degree, very: min-ku-ak-a very ripe; $\mathbf{k a} \cdot \mathbf{- k u}-$ ak-a very bitter; sin-ku-ak-a want very much. (3.342)

ku-ak-ku-ak $a d v$. with the mouth open and gasping when nearly dead and when the jang-gi (soul, life force) is about to leave the body; the similar mouth gesture of fish. (6.79)

ku-bi, kup $a d v$. very, (see kup). (3.76)

-ku-ja- $a a$. not yet: nom--ku-ja not yet soft; man-ku-ja can't yet. $(0.41)$

ku-ku-ga-ri $a d v$. lots of crying, as by a child. (7.29)

ku-mil-ong-a, ko-mil-ong-a, ku-mil-ong dong-a vi. naked above the waist. (6.552)

ku-min-a vi. eaten enough, tired of eating. (5.48)

ku-na, ko-na $n$. bottom corner of a basket; corner of a piece of paper, a field, a dike, etc. $<$ B (5.854)

ku-pa gro, ka-pa gro $n$. a legal case. (8.13)

ku-rak-a vi. break, fall to pieces, as an earthen wall. ku-rak- cls. classifier for the split pieces of a length of bamboo, for lengthwise pieces of papaya, etc. -ku-rak- aa. into pieces, separate: be•-ku-rak-a broken into pieces; pit-ku-rak-a split into pieces. (9.212)

ku-ri $n$. a low or deep spot in a pond, low place in rice fields where water collects; small tank that goes dry in the dry season; place, as in some place names. $<$ B $(4.253)$

Ku-ri mit-e $n$. a mit-e (spirit) that makes people crazy. (8.54)

ku-ro, ku-ru $n$. heartwood, hard central wood. (4.673)

ku-rom-ku-tom $a d v$. so sick and weak that getting up and walking is very difficult. (8.93)

ku-ru-ak $n$. red eagle, dives for fish, shrimp, snakes, (Beng. igol pa-ki). $(4.43)$ 
ku-si $n$. happiness. $<\mathrm{B}(7.721)$

ku-ta $n$. mud, mud house, mud construction. $<\mathrm{B}(5.631)$

ku-ti $n$. horizontal wooden peg embedded in a mud wall to which the roof is tied: nang-gol ku-ti handle of a plow. $<\mathrm{B}(5.632)$

ku-tip, ko-tip $n$. turban, (see ko-tip). (6.522)

ku- - cls. classifier for mouths, words, bits of language; mouthsful of solid foods; doors, gongs; single split sticks of firewood. $\mathbf{k u} \cdot \mathbf{-}$. prefix for the mouth and surrounding area: ku-sik mouth; ku-sim-ang beard; ku•-chil lip. (1.37)

$\mathbf{k u} \cdot \mathbf{a}$, ko-a $v t$. draw water up from an open well, dip out water. (9.277)

ku·-ak-a vt. scratch to hurt, with claws or nails, by animals or people; claw like a cat; scrape off the excess wet mud of a wall during house construction. (9.554)

ku-ak-chrak-a vt. scratch badly: meng-gong ang-ko ku-ak-chrak-a the cat scratched me badly. (8.955)

ku•-ak-ma $a d v$. spoken language, as contrasted with writing or singing. $(7.12)$

ku-ang-dak-e chu-a $v p h$. sleep with an open mouth. (9.343)

ku·-bi-bal, song-ni ku•-bi-bal $n$. ordinary way of speaking, village dialect. (7.11)

ku•chak-a vi. respond, answer, agree, promise. (7.32)

ku'-chi $n$. saliva; bubbles. (6.29)

ku•-chi kan-a, ku•-chi ron-a vph. give advice. (7.37)

$\mathbf{k u} \cdot \mathbf{- c h i - a , ~ k u}$-sik ku•-chi-a $v t$. crave, long for, esp. for tobacco. (5.48)

ku•-chil $n$. lip. (6.22)

ku-chil bret-a vph. chapped lips, (lit. lip bursts). (8.953)

ku-chil-dang-dang $a d v$. having big lips. (6.42)

ku-chim-mong-a vi. discuss carefully. (7.31)

ku•-ching-a vt. threaten. (8.36)

ku'-chok-a vi. pointed, with a protruding mouth, as the nose of an animal such as a dog, the end of a pen, of a crescent moon, of a field with pointed corners. ku-chok-chok $a d v$. (2.312)

ku•-chon-a vi. with a quiet, soft voice; small, of mouths. (7.12)

$\mathbf{k u}^{*}$-chot-a $v t$. make a smacking noise to a child to soothe it. echo in ku•-dim-a ku•-chot-a kiss, smack. (9.44)

ku'-chot-chot $a d v$. small-mouthed, of a person, basket, pot. (6.42)

ku•-dal-a vi. big, of mouths, firewood; ku•-dal-e bol-a speak loudly. (2.211)

ku•-dal-it-a vt. enlarge the mouth of a basket, cause to flair. (5.856)

ku-dam-bot-a vi. having a flat face, flat chin. (6.42)

$\mathbf{k u} \cdot \mathbf{- d e p - a}, \mathbf{k u} \cdot \mathbf{- c h i l} \mathbf{k u}-\mathbf{d e p}-\mathbf{a}$ vi. have a flat mouth. (6.42)

ku'-dim-a vt. kiss, as a baby; make a smacking noise to a child to soothe it. $(9.44)$ 
ku'-dim-bok $n$. chin. (6.22)

$\mathbf{k u} \cdot \mathbf{- e n g}-\mathbf{k u} \cdot \mathbf{- c h o k} a d v$. a shape with sharp corners, as a triangle, diamond. $(2.313)$

ku-gi-sep ang-ki-sa dak-a $n$. rash at corner of mouth, cold sore. $(8.953)$

ku'-grip-a $v t$. cover the mouth of a pot. (5.46)

$\mathbf{k u} \cdot \mathbf{- m a - k u \cdot - m a} a d v$. with a full mouth. (5.472)

$\mathbf{k u} \cdot \mathbf{- m a - s a} a d v$. echo in $\mathbf{k u} \cdot \mathbf{- m i}-\mathbf{s i} \mathbf{k u} \cdot \mathbf{- m i}-\mathbf{s a}$ whisper. (7.15)

$\mathbf{k u} \cdot \mathbf{- m a n}-\mathbf{a} v i$. able to speak; often negative: $\mathbf{k u} \cdot \mathbf{- m a n}-\mathbf{j a}$ speak badly, stutter. (7.14)

ku-man-si-a $v t$. pledge to give a man-sa (ceremony) if a sick person gets well. (8.566)

ku-mang $n$. end, of a bamboo strip, a piece of thread; point of a pen; mouth of a gun. (5.87)

ku-mang sik-a $v p h$. insert an end into something, as to thread a needle. (6.598)

ku'-mi-si-a vi. whisper. (7.15)

ku'-mik-chet-a, ku'-mit-chet-a, mik-chet-a vi. swear, pledge, promise. $(7.33)$

ku-min-dik-a vi. alone, with no one to talk to. (7.743)

ku'-mit-tip-a vi. close lips, purse lips. (9.44)

ku'-mit-tip-e chu-a vph. sleep with a closed mouth. (9.343)

ku'-mong-a vi. agree, talk over and come to an agreement discuss. (7.33)

ku-neng-a vi. tired voice. (7.29)

ku-pa $n$. language, speech, voice; language used at a legal meeting. (7.12)

ku·-pa-a vi. speak at a legal meeting, have a dialogue, talk with, converse. (7.31)

ku'-pret-a vi. speak fluently, speak without shyness. (7.14)

ku-ra-chak-a vi. agree, promise; pledge, as pledge to give a sacrafice (man-sa). (7.33)

ku'-ra-ma $n$. voice, quality of one's voice, way of talking. (7.15)

ku'-rak-a, ku-rak-e bol-a vi. shout, speak loudly, (lit. strong in language). (7.15)

ku-rang $n$. voice: bi-ni $\mathbf{k u} \cdot-$ rang $\mathbf{k i n}-\mathbf{a}-\mathbf{t o}-\mathbf{a}$ his voice is beautiful. $(7.15)$

ku'-rang, wa'-ku'-rang $n$. a length of bamboo split in half. (5.639)

$\mathbf{k u} \cdot$-rang-ku'-dang $a d v$. speak angrily, in an ugly way. (7.766)

ku-rit-chu-a vi. repeat what another person has said, gossip, rumor. (7.31)

ku'-ron-a $v t$. warn, advise, give information. (7.37)

ku-sep, ku-gi-sep $n$. corner of the mouth. (6.22)

ku'-sik n. mouth; language; opening of a pot, etc.; front end of a worm; pal $\mathbf{k u}$-sik tip of a plow blade. (6.22) 
ku'-sim-ang $n$. beard; tassel of a corn cob. (6.25)

ku'-sim-ang chap-cheng $n$. small chin beard. (6.25)

ku'-sim-ok-a vi. whistle. (7.29)

ku-sol sal-a vi. speak or sing in a beautiful slow style; dragged out, streched out. (8.83)

ku-srip-a $v t$. swirl water in the mouth to rinse it. (9.44)

ku'-tip pat-a vph. give symbol of power, create a headman, (lit. wrap the turban). (8.12)

ku'-wang $n$. crazy person, having mouth agape. (8.92)

ku'-wang-a, ku'-sik-ko wang-a $v p h$. let the mouth hang open, as in surprise or by a crazy person; large-mouthed, of baskets. (9.44)

ku'-wang-e chu-a $v p h$. sleep with one's mouth open. (9.343)

ku'-wat-a vi. promise, as promise to give a man-sa (sacrifice, ceremony). (7.33)

ku-weng-a vi. curved, of a mouth when twisted to the side or when crying; curved of a crescent moon. ku-weng-weng adv. (9.44)

$\mathbf{k u}$-weng-brak-brak $a d v$. with edge broken and out of shape, as the mouth of a pot, etc. (9.282)

kui-cha, kut-chi-a $n$. eel. (4.454)

kul, pul $n$. masonry, brick, or iron bridge for people and vehicles. $<\mathrm{B}$ (5.646)

kul-i-a vi. to be open, as a box, shop. <B (9.211)

kun-da $n$. the back part of a gun that rests against the shoulder, gun stock. (5.845)

kung-chut $n$. bright red plant, about two feet high with vivid colored flower stalks. (4.656)

kup, ku-bi adv. very. $<$ B (3.76)

kus $n$. fish-catching fork, with a long bamboo handle. $<$ B (5.843)

kut $n$. coat. $<\mathrm{E}(6.527)$

kut me-ris $n$. civil marriage, court marriage, a marriage performed by a judge. $<$ E $(6.651)$

kut-chi-a, kui-cha $n$. eel. (4.454)

kut-chi-a-gran $n$. dried eel. (5.401)

la-gi-a, na-gi-a $v t$. need. $<\mathrm{B}(7.726)$

la-ti $n$. a pole used as a weapon. $<$ B (5.833)

lain sal-a vi. make a row, mark out a row, as when planting pineapple, etc. (5.112)

lait, tos-lait $n$. flashlight, torch. $<\mathrm{E}(5.838)$

lak, lo-ko num. one hundred thousand. <B (3.38994)

lal-sak $n$. edible spinach-like leaves of a small reddish plant. $<\mathrm{B}(5.23)$

lang-gol, nang-gol $n$. plow. $<\mathrm{B}(5.831)$

lap $n$. benefit; profit, as profit in business. $<$ B (8.442) 
lap cha'-a $v p h$. take a profit. $<$ B (8.442)

lau, rau $n$. gourd, a long edible squash that hangs from a climbing vine. $<\mathrm{B}(5.23)$

le-bu $n$. large green lime. $<\mathrm{B}(5.22)$

le-chu, li-chu $n$. litchi fruit. $<$ B (5.22)

le-la-le-la $a d v$. loose, as of a tool handle, table leg; lacking stiffness, limp, as of a paralyzed hand. (2.23)

lek-gu-a, lek-gu-gu-a vt. tickle, (same as rem-bu-bu-a, jik-jik-a). (3.64)

lek-ka, le-ha $n$. written things. $<\mathrm{B}(7.28)$

lek-ka-po-ra $n$. reading and writing, literacy. $<\mathrm{B}(7.28)$

lek-ki-a $v t$. write. $<$ B (7.28)

leng-got $n$. man's underwear, similar to reng-ti (old fashioned loincloth). $<\mathrm{B}(6.527)$

leng-ti, reng-ti $n$. loin cloth. $<\mathrm{B}(6.527)$

lep $n$. thick padded quilt with cotton stuffing. $<\mathrm{B}(6.591)$

li-chu, le-chu $n$. litchi fruit. $<\mathrm{B}(5.22)$

li-li-pul $n$. lily, a decorative flower. $<\mathrm{E}(5.28)$

li-pi stiik $n$. lipstick. $<\mathrm{E}(6.565)$

li-lap-a vi. lightning. (4.344)

liik $n$. leak, of a bicycle tire. $<\mathrm{E}(5.872)$

lil--lil-a vi. make noise, of thunder, an engine. (4.342)

long-go $n$. cloves, a spice. $<\mathrm{B}(5.405)$

long-pen $n$. long pants. $<\mathrm{E}(6.527)$

lunggi $n$. man's tube-like skirt. $<\mathrm{B}(6.527)$

lus-kan $n$. loss, opposite of profit. $<\mathrm{B}(8.442)$

$\mathbf{m}$ int. a murmur made that shows agreement to an order or request, it may begin with a nondescript vowel. (1.24)

-ma dns. female human. (6.821)

-ma dns. big, large: ta-ma a large edible tuber; mi-ma a big grained variety of rice; ja--dil-ma large roots; bi-rot-a-ma large pimple. (2.213)

ma int. what?, what did you say? (0.64)

ma $n$. matrilineal kin group, (same as ma-chong). (6.63)

-ma $t s$. question marker that forms yes-no questions: neng-jok-ma? are you tired? ma? qw. what (did you say)? (0.31)

ma-chang $n$. platform on which a house is built. (5.637)

ma-da-gong-gil-ek $n$. an egg-eating forest-dwelling bird, bigger than common myna. (4.43)

ma-ga $n$. scar, as from wound, vaccination or from the skin disease called hang-ki-sa-a; skin blemish; worn place on bark where cows have been tied. (8.955)

ma-gi-a $v t$. beg, ask for alms. $<$ B (9.72)

ma-gi-a-ri $n$. beggar. $<\mathrm{B}(8.41)$ 
ma-gin-a $a d v$. working without money wages, but often with food provided, as with a work group for building a roof where friends are given food and rice beer but no money. $<\mathrm{B}(8.435)$

ma-ha-jon, mo-ha-jon $n$. money lender. $<\mathrm{B}(8.41)$

ma-ha-ri $n$. kin group, lineage, named group, such as Nok-rek, Chambu-gong, etc. (6.63)

ma-je-ma-je, ma-ji-ma-ji $a d v$. now and then, sometimes. <B (2.71)

ma-ji-la $n$. middle child, middle one. $<\mathrm{B}(6.64)$

ma-ju-ru $n$. a wild striped forest cat that is bigger than a gri-taog but not as big as mat-cha-am-gip-a. (4.416)

ma-kan-chi $n$. tree with 20-30 cm. lozenge shaped leaves. (4.632)

ma-ma, ma-ma-gip-a, ma-ma-tang $n$. mother's brother. <B (6.611) ma-ma-sa $n$. mother's brother and his sister's child. (6.62)

ma-mang $a d v$. only, just, merely: i-ang-a ma-mang (he) simply went. $(3.344)$

ma-mil-a $n$. legal case, especially one that goes to a government court. $<\mathrm{B}(8.13)$

ma-ming-ba, ma-mung-ba $n$. something; nothing, with a negative verb: ma-ming-ba dong-ja it is nothing, it doesn't matter. (3.346)

ma-mon-chi $n$. very small red tick-like insect that bites the naval, eyes, and ears, and causes itching. (4.468)

ma-ni, a-ni $n$. father's sister, mother's brother's wife, mother-in-law. (6.612)

ma-ni-a vt. follow instructions, obey, do as another suggests; worship. $(9.75)$

ma-ni-sa, a-ni-sa $n$. a mother-in-law and her son- or daughter-in-law. (6.62)

ma-ni-tang dong-gip-eng dong-gru $n$. the belt and sword of the constellation Orion, (lit. mother-in-law, across, long, implies that the son-inlaw is carrying his mother-in-law). (4.212)

ma-ra-a vt. knock off grain, by cattle. <B (5.451)

ma-rai-a vi. whine, seek attention, pester, as a hungry baby or a dog that rubs up against someone. (9.78)

ma-rak-ha $n$. barking deer. (4.419)

ma-rang $n$. pollution, taboo, blood, misfortune, evil, crime. (8.52)

ma-rang rok-a vph. drive away ma-rang (pollution), drive away evil, a kind of sacrifice that requires an egg and a bearded bamboo. (8.562)

ma-rang wat-a $v p h$. get rid of pollution (ma-rang) as by an offering of an egg. (8.562)

ma-reng-ga $n$. a club to hit someone with, walking stick larger than pa-jun. (5.833)

ma-ri-a $v t$. kill, murder. $<\mathrm{B}(6.79)$ 
ma-ri-bi-du $n$. the largest vine that grows in Modhupur, woody and as thick as a person's leg, it has $25 \mathrm{~cm}$. leaves, red sap, and grows wild in the forest: ma-ri-bi-du sek-i the red sap of ma-ri-bi-du that is drunk as a cure for watery diarrhea. (4.655)

ma-star $n$. teacher. $<\mathrm{E}(8.6)$

ma-sul-eng-ga $n$. kingfisher, small blue bird that catches fish. $<$ B (4.43) ma- cls. classifier for groups of chicks with a mother hen. (1.35)

ma- cls. classifier for hundreds, generally for takas, the Bangladeshi currency unit. (1.35)

ma- $c l s$. classifier for the number of pieces crossed together by a perpendicular piece in weaving a basket, for small pieces of areca nut. (1.37)

ma- $q w$. combining form of $\mathbf{m u}$-a what? ma-ko ra-ba-jok? what (did you) bring?; ma'-ming with what?; ma'-ni sal? on what day? (1.21)

ma-a-pa-a $n$. parents, mother and father. (6.62)

ma-am-a vi. groan in suffering, make shivering sounds. (7.29)

ma'-ang, ma`-ang-di'-ang $a d v$. craving, longing for, as for tobacco; searching for. (7.725)

ma-at-a vt. divide, apportion, property, meat, money, etc. (8.427)

ma'-bai, ma'-ki, mai-ba int. hesitation form used when collecting one's thoughts. (0.64)

ma’-bak-a, na·-bak-a vi. dirty, soiled from dust, muck, etc., of people and things. (3.716)

ma•-chong $n$. kin group, lineage, named group, such as Nok-rek, Chambu-gong, etc. (6.63)

ma-chong $n$. vertical bamboo strips of a basket, vertical cane pieces of a sling for a pot. (5.854)

ma'-de, ma'-di, $\mathbf{a}^{\bullet}-\mathbf{d e}, \mathbf{a}^{\bullet}-\mathbf{d i} \quad n$. mother's younger sister, father's younger brother's wife, aunt, stepmother. (6.612)

ma-dok-a $v t$. echo in ma'-ring-a ma-dok-a miss someone, long for. $(7.743)$

ma-dong $n$. incest, sexual relations or marriage within a named lineage. (6.657)

ma'-drang $n$. lineage, (same as ma•-chong). (6.63)

ma'-gin-i $n$. weaving system in which each thread or bamboo strip crosses two perpendicular pieces together. (6.598)

ma・-gip-a n. mother. (6.612)

ma'-git-tam $n$. the usual basketry weave where a strip crosses over or under groups of three perpendicular strips. (5.856)

ma-jang-chi $n$. middle child, neither the youngest nor oldest child. (6.64)

ma-jim-a vt. make wet, as to settle dust. (9.272)

ma-jong, a'-jong $n$. mother's older sister, father's older brother's wife. $(6.612)$ 
ma-ki int. a hesitation form used when collecting one's thoughts. (0.64) ma'-man-ti $n$. everything, all sorts, lots, of various kinds, (A•chik). $(3.343)$

ma-mu-ri $n$. thong that encircles the end of a drum and that holds the end pieces. (8.85)

ma`-na, mo`na $q w$. why? for what?: mo`-na grap-ing-a? why (are you) crying? (A•chik: mai-na). (1.21)

ma•-ning-sa $n$. mother and child. (6.62)

ma-nok $n$. members of one's own lineage, especially the women: mother, sisters, mother's sisters, etc. (6.631)

ma`-pil-a $v t$. make a pattern in basket weaving that zig zags, first outward and then back inward. (5.856)

ma•-ring-a vi. miss, be lonely for, cry for someone, want to see someone. $(7.743)$

ma-sa $n$. simple over-under weave, tabby weave, used for rough openwork baskets such as a kok-kreng. (5.856)

ma•-sa num. one hundred. (3.3799)

ma・-si-a vt. understand, $(\mathrm{A} \cdot$ chik $) .(7.41)$

ma-si-a, ba-si-a $v t$. last stage of husking rice when one gets rid of bi-ma (husks that have no grains) by picking them out by hand. (5.451)

ma•-si-a ma'-tat-a vi. angry. (7.766)

ma`-sik-a, ma•-sik-a-sik-a vi. belonging to the same ma•-chong (lineage). (6.657)

ma·-skap-a vi. sticky. (3.668)

ma・-su $n$. cow, bull, cattle, (A•chik: mat-chu). (5.33)

ma'-su be'-en $n$. beef, (lit. cattle-meat). (5.401)

ma`-su gen-da, ma•-su pi--sa, ma•-su ba-sur $n$. calf. (5.33)

ma`-su ma•-rong $n$. wild cattle. (4.419)

ma·-su mat-ti $n$. cattle. (4.40)

ma-su ri-sip-il $n$. tree with $12 \mathrm{~cm}$. leaflets on $40 \mathrm{~cm}$. forked leaf, (lit. cattle testicles), (Beng. pi-tras). (4.632)

ma·-su-a-gong $n$. smelly $30 \mathrm{~cm}$. tall weed with $10 \mathrm{~cm}$. long, very skinny pods and leaves with six leaflets. (4.656)

ma•-su-ga-ri $n$. ox cart. (5.86)

ma•-su-ke•-em-a, ma•-su-ki n. cow dung. (4.475)

ma-tap-a vt. paste, stick, paste up; press onto skin as medicine. (9.63)

mai qw. what? (alternate of $\mathbf{m u}-\mathbf{a}$, (primarily A•chik). (1.21)

-mai ts. contraction of past marker -ming and emphatic -ai. (0.314)

mai-ba int. hesitation form use when collecting one's thoughts, well. $(0.64)$

mai-ja vi. echo in dong-ja mai-ja don't have, doesn't exist, or with any other negative verb. $(0.5)$ 
mai-jing-jing $n$. noisy, finger-sized insect. (4.469)

mai-mai $n$. with a negative verb: nothing: mai-mai dong-ja there is nothing. $q w$. what all?: mai-mai nik-jok? what all did (you) see? (3.346)

mai-na $q w$. why? what for? (A•chik). (1.21)

Mak $n$. a Bengali month that overlaps January and February. $<$ B (2.831)

mak-bil $n$. bear. (4.419)

mak-kal $n$. a shrub whose dark $20 \mathrm{~cm}$. long leaves are used for fish poison. (5.843)

mak-kal-ri-gi $n$. vine that causes severely irritated skin if touched. (4.655)

mak-kre, mak-kri $n$. macaque, monkey. (4.418)

mal gal-a vph. the first washing of new cloth, getting rid of the starch. (6.57)

mal-a $n$. necklace, neck ornament; circular cane piece at the top of a basket. $<\mathrm{B}(6.563)$

mal-a vi. crawl, of children, lizards, bugs, etc.; want to be with, come to lovingly, as a child comes to his grandfather, or an animal pet to its owner. (9.319)

mal-a vt. put on jewelry, put on around neck, wear at neck. $<\mathrm{B}(6.53)$

mal-a-ni sam $n$. love potion, to make someone want to be with another, (lit. medicine to make someone crawl). (7.722)

mal-ak-mal-ak adv. crave water, thirsty. (5.48)

mal-am-a vi. crawl, of children, lizards, bugs, etc. (9.319)

mal-dim-dim-a vi. crawl quickly. (9.319)

mal-et-a vt. tempt, attract, (lit. cause to crawl). (7.38)

mal-gek-a vi. be very tame, of an animal. (4.482)

mal-gip-a $n$. crawlers, crawling animals, snakes, insects, etc. (4.461)

mal-iik $n$. owner, boss. $<\mathrm{B}(8.41)$

mal-mok-a $v t$. encourage, soothe, give comfort, as to a fussing child. (7.37)

mal-ru-rat-a vi. crawl back and forth. (9.319)

mam, mam-mam $n$. baby word for rice. (5.403)

man-dal $n$. small tree with large poplar-like leaves and colorful flowers, with thorns on the trunk and branches; used to build fences. (4.632)

man-de, man-di $n$. person, human being; specifically, a member of the Garo ethnic group. cls. classifier for the height of a person: man-de gin-i twice as tall as a person. (6.821)

man-de ba-ring $n$. a small type of eggplant that was cultivated in the past. (5.23)

man-de-bi-ma, man-de-bi-pa $n$. animal-woman, animal-man, a way of joking by adding suffixes ordinarily used for animals to the word for person. (7.26) 
man-de-gil-we $n$. a shy person, one who does not want to show his face, like a gil-we, an animal known for its shyness. (7.745)

man-de-ku, man-di-ku, man-de-ku•-sik $n$. the Mandi language, the Garo language. (7.11)

man-de-ming jak-ming cls. classifier for the height from the ground to the tip of a person's upstretched fingers, (lit. person-with-arm-with). (1.36)

man-de-ska, man-di-ska $n$. fellow humans, fellow Garos. (6.821)

man-di, man-de $n$. person, human being; specifically, a member of the Garo ethnic group. (6.821)

man-sa $n$. a ceremony regarded as Christian, but much like a Sangsarek sacrifice, that may be done to cure a disease, or in fulfillment of a promise to give thanks when a sick person has recovered. $<\mathrm{B}(8.566)$

man-teng-gin-a $n$. a stick, a stick used for hitting. (5.833)

man-ti, man-chi $n$. yeast for making rice beer, $(\mathrm{A} \cdot \mathrm{pal})$. (5.405)

man॰-a vi. be able, can; find, get, accomplish, achieve, finish: bal-wa man--ing-a wind blows; sol-di man·-ing-a have a cold. -man•- $a a$. be able accomplish, finish: $\mathbf{k u} \cdot \mathbf{- m a n - a}$ able to speak, speak well; bil-man-a have the strength for; nik-man-a discover, notice. (7.55)

man·-chak-a vi. succeed against opposition, overcome, manage; win at sports. (7.55)

man'-chek-chek-a vt. can do well. (7.55)

man-dik-a vi. be disturbed, annoyed, bothered, as by pestering children; having too much work. man•-dik-man'-si dak-a $a d v$. (9.78)

man'-pit-chi vi. get a little, not much. (7.55)

man-pran-ja vi. can't get everything finished. (7.55)

man-prong-a vt. blow up, blow around, of dust, cotton fluff, etc. (4.36)

man-rik-a vt. get back something given away or lost, recover; obtain, receive, keep; inherit. (9.11)

man'-ring-a $v t$. do at the right time; often negative: man•-ring-ja late, at the wrong time. (9.531)

man-sit-tong-a vt. get everything. (9.531)

-mang ns. plural marker for a few pronouns, $(\mathrm{A} \cdot \mathrm{chik})$ : na·-si-mang you plu.; bi-si-mang they; u-a-mang those. (0.11)

mang- cls. classifier for animals, including mammals, birds, insects, etc., occasionally for ghosts, spirits: tam-pi mang-sa one fly. (1.31)

mang-cha-a vt. angry at, wanting to fight, of animals or people: ang-ko a-chak mang-cha-ing-a the dog is angry at me. (8.36)

mang-gi-si, man-di mang-gi-si $n$. dead person, dead body, corpse. (6.79)

mang-got $n$. small stick such as might be used for a cattle prod; walking stick, cane. (5.833) 
mang-got sol-a, mang-got $\mathbf{r a}^{\bullet}-\mathbf{a}$ wph. give symbol of power, create a headman, (lit. carve the staff, take the staff). (8.12)

mang-gra-a vi. angry, wanting to fight, of people and animals. (7.766)

mang-gu-ak $n$. a small, nonbiting centipede. (4.467)

mang-gu-ak git-chak $n$. a small type of centipede, that sometimes lives in roofs. (4.467)

mang-gu-ak jol-si $n$. a small and thin, but long centipede that lives in the ground. (4.467)

mang-gu-ak mi-bram $n$. a small type of centipede, that appears in large numbers. (4.467)

mang-gu-ak pil-dep $n$. small black centipede. (4.467)

mang-gu-ak ron-da $n$. a large type of centipede. (4.467)

mang-kal-a vt. play games, tease, fuss with, fool around, do mischief; annoy, disturb, as children do: di·-sa-ko mang-kal-a joke, fool around with the child. (9.78)

mang-kan-dik-a vi. short in the body, of animals. (2.211)

mang-meng-a vi. irritated, tired, impatient, fussy, whining. (9.78)

mang-meng-dil-a $v t$. annoy, make impatient. (9.78)

mang-meng-et-na-jok $v t$. about to reach the irritating, (complaining) stage, a stage of child development. (6.78)

mang-meng-mang-chip $a d v$. be annoyed, pestered. (9.78)

mang-rak-a vi. strong in body, healthy, without disease or weakness. (8.91)

mang-ri-a vi. go to a funeral in a procession, carrying a pig, rice beer, drums, etc. (6.79)

mang-ro-a vi. long in the body, of animals; long in the torso, of people. (2.211)

mang-ru-dam $n$. graveyard. (6.79)

mang-sok-a vi. plan, intend. echo in mang-ri-a mang-sok-a go to a funeral with pig, beer, drums, etc. (7.48)

mang-song-a, ming-song-a vt. plan, intend. (7.48)

mang-tip $n$. a small caterpillar with stinging hairs that irritate the skin when touched, smaller than a jong-su. (4.467)

mang-wa-a vi. miss someone sadly, mourn for a dead person. (7.743)

map-pru $n$. large long-tailed forest lizard that lives in trees. (4.441)

mas n. month. <B (2.53)

mat $n$. squirrel. (4.419)

mat $n$. playing field. $<$ B (5.112)

mat- $c p$. prefix for some mammals: mat-cha tiger; mat-ma water buf-

falo; mat-chok deer; mat-ram a fish-eating wild cat. (0.17)

mat-a vi. sharp, cut, scarred. vt. wound, make a slicing cut. (3.666)

mat-bor $n$. headman, village leader, head person. $<$ B (8.12) 
mat-bring, mat-brung, mat-bu-rung $n$. forest animals, esp. mammals, but not including birds or insects. (4.40)

mat-cha $n$. tiger, leopard, any of several kinds of large cats. (4.416)

mat-cha dal-gip-a, mat-cha-am-gip-a $n$. tiger, largest of the wild cats. (4.416)

mat-cha ma-jang-chi $n$. middle sized mat-cha, (same as mat-chasal-rong). (4.416)

mat-cha mak-bil $n$. wild animals, (lit. tigers and bears). (4.40)

mat-cha-a-rek $n$. leopard, the smallest of three sizes of mat-cha, spotted. (4.416)

mat-cha-a-ro-pa $n$. Royal Bengal tiger, the largest of the tigers, (same as mat-cha-am-gip-a). (4.416)

mat-cha-am-gip-a $n$. tiger, the largest of three sizes of mat-cha, striped. (4.416)

mat-cha-du, mat-cha-bet, mat-cha-brek $n$. a human being who is able to change into a tiger. (8.51)

mat-cha-ki-brin-a vi. black mixed with gray or white, of hair, (lit. mixed tiger dung). (3.538)

mat-cha-sal-rong $n$. the middle-sized of the three large cats. (4.416)

Mat-chi $n$. a subgroup of Garos who have never been seen by the Modhupur people, but who are said never to wash and to eat cats and dogs. Some believe them to have lived in or near to Modhupur at an earlier time. (6.89)

mat-chin-da-ri-a vt. cut slightly, cut a little. (8.955)

mat-chok $n$. deer. (4.419)

mat-chok-grong te-rik $n$. kind of banana that is sometimes cooked, but sometimes eaten raw. (5.22)

mat-chot-a vt. finished, done, (A·chik). (9.14)

mat-chrak-a vt. scratch, as by a cat. (8.955)

mat-chrit-a, mat-srit-a vt. cut slightly, cut a little; scratch, by people or animals. (8.955)

mat-chu $n$. cow, cattle, $(\mathrm{A} \cdot$ chik $) .(5.33)$

mat-chu-ra $n$. stopper for a bottle, cork, bottle cap. (5.434)

mat-chu-reng $n$. a small kind of squirrel with a long snout. (4.419)

mat-chu-ri $n$. civet cat, a small, red and black, long tailed and bad smelling wild cat. (4.416)

mat-chu-ri gri-tong $n$. big variety of mat-chu-ri. (4.416)

mat-chu-ri kal-dek $n$. small variety of mat-chu-ri. (4.416)

mat-chu-ri wa-rik-i $n$. smallest variety of mat-chu-ri. (4.416)

mat-da-bu $n$. a large kind of squirrel. (4.419)

mat-ek-a n. rabbit, (same as kor-gus). (4.419)

mat-gil $n$. cowhide that is stretched over the end of a drum. (8.85) 
mat-kil-ot-a $v t$. cut a little, not much. (9.573)

mat-ma $n$. water buffalo, wild buffalo. (5.35)

mat-ma ra-ma $n$. Milky Way, (lit. buffalo-road). (4.212)

mat-mat-chi $n$. porcupine. (4.419)

mat-mi-mang $n$. a me'-mang (ghost) that frightens people, especially on the road at night. (8.54)

mat-ram $n$. otter, a one or one and a half foot high fish-eating mammal. (4.419)

mat-ta $n$. digging stick. (5.831)

mat-te, mat-ti $n$. a loner, human or animal, rogue male that stays alone. $n$. echo in ma-su mat-te cow, cattle. (5.33)

mat-tin $n$. bundle of a special meat preparation made from a strip from the back of the animal that is rolled in a banana leaf, and may be eaten at a sacrifice and offered to a spirit. (5.401)

mat-tul $n$. large round hat, worn to keep off rain. $<\mathrm{B}(6.522)$

mat-tul $n$. screwdriver. $<\mathrm{B}(5.838)$

mat-u-a vt. greedy, covet, yearn for a person or thing, fall in love. (7.723) mat-u-kok $n$. a greedy person, one who wants everything. (7.724)

me-di $n$. agreement to rent land, rent paid on land. $<\mathrm{B}(8.425)$

me-di jok-a $v p h$. end an agreement to rent land, stop renting. (8.425)

me-di ra-a $v p h$. rent land from another, take land for money payment. (8.425)

me-di ron-a vph. rent land to another for money payment, rent out land, let. (8.425)

me-ga-ru, mi-ga-ru $n$. an edible grain, Job's-tears or pop sorghum. $(5.23)$

me-ja an`-tam-o, me-ja-o an॰-tam-o $l w$. last evening, yesterday evening. (2.62)

me-ja wal-ni, me-ja-o wal-ni $a d v$. yesterday morning. (2.62)

me-ja wal-o, me-ja-o wal-o $l w$. last night. (2.62)

me-ja-o, mi-ja-o $n$. yesterday. (2.65)

me-ja-o-na skang $n$. day before yesterday. (2.65)

me-kop, mi-kop n. maize. (5.23)

me-li-a, mi-li-a vi. get along well, not fight, peaceful, good relationship; go together well, of things, words. $<\mathrm{B}(8.32)$

me-rong, mi-rong $n$. husked rice. (5.25)

me-rong-chek-chek $n$. rice that is free from husks, well cleaned rice. $(5.25)$

me-sa-a $v t$. echo in grik-a me-sa-a dance with sword and shield. (8.83) me-sak-a, mi-sak-a vi. itch, irritated, have an eruption on skin, burn from touching the hairs of a mang-tip worm or mak-kal-ri-gi plant: git-dok me-sak-a feeling in throat after eating ta--ring (arum). (8.94) 
me-se, mi-si $n$. mouse, rat. (4.419)

me-se da-bu $n$. rat, large sized me-se that makes tunnels in the ground like a mole. (4.419)

me-se rong-kan-tek $n$. mouse, small sized me-se that lives in the roofs of houses. (4.419)

me-se wa-gong $n$. a kind of rat that is smaller than a me-se da-bu. (4.419)

me-seng-a, mi-seng-a vi. sour. (3.682)

me-siin $n$. machine, such as a rice mill, diesel pump. <E (5.838)

me•-a, mi・-a $n$. edible bamboo shoots. (5.23)

me•-a, mi-a n. man, male; me-a pi·-sa male child, boy. (6.821)

me•-a-sa, mi-a-sa $n$. man, adult male. (6.821)

me•-chik, mi•-chik, me•-chik-sa $n$. woman, adult female; me•-chik pi-sa woman-child, girl. (6.821)

me•chik be-wal vi. menstruation, (lit. woman-habit). (6.72)

me-dik, mi-dik $n$. rice cooking pot. (5.432)

me•-dik-sam-dik, mi•-dik-sam-dik $n$. cooking pots and pans, (lit. ricepot curry-pot). (5.432)

me•-gon, mi-gon $n$. stick for stirring rice. (5.438)

me-mang, mi-mang $n$. ghost, beings that are invisible but that frighten people. (8.54)

me'-mang gam, mi-mang gam $n$. funeral, ceremony for the dead, (lit. ghost work). (8.562)

me•-mang-ka•-chi, mi-mang-ka•-chi $n$. bush with skinny half-meter prickly stalks with slightly red tipped ends, small scattered leaves and .5 cm. seeds scattered up and down the stalks; used as medicine to apply to sore limbs. (4.64)

me•tra, mi•-tra $n$. unmarried woman, young woman, adolescent girl. (6.824)

mel-e-ri-a n. malaria. $<\mathrm{E}(8.92)$

mel-e-ri-a bi-pang $n$. tall skinny tree with somewhat sparse leaves that is planted by forest department; a kind of eucalyptus. $<\mathrm{B}(5.22)$

mel-a vi. slightly rotten with a somewhat bitter or almost burning taste, like sap or citrus peel, of bad fruit or bad rice beer. (3.682)

men-da $n$. sour vegetable with large buds and edible leaves and outer flower petals. (5.23)

men-di-ka-ta $n$. small tree with small opposite leaves, that is planted and cut off to make fences. $<\mathrm{B}(4.632)$

men-di-pul $n$. ornamental plant with a tall stiff woody stalk. (5.28)

men-du $n$. a yellow flowered pea with edible seeds. (5.23)

meng-chut $n$. mouse-sized animal with a long nose, stink-rat, shrew. (4.419) 
meng-go n. cat, (A•chik). (5.35)

meng-gong $n$. cat, (A•chik: meng-go). (5.35)

meng-meng-ka-ri $n$. small bee-like insect, that makes no honey and does not sting, but makes lots of buzzing sound. (4.463)

mes $n$. match. $<\mathrm{E}(5.423)$

mes bi-king $n$. matchbox. (5.423)

mi $n$. rice plant, unhusked rice, cooked rice. (5.25)

mi-a-tam $n$. evening rice meal. (5.477)

mi-bi-pang, mi-pang $n$. rice plant. (5.25)

mi-chi $n$. liquid from curry, juice, gravy. (5.409)

mi-dam-bek $n$. very soft boiled rice, rice cooked with lots of water that can be eaten with gu-ru-ma (locally made brown sugar) or milk. (5.403) mi-dap $n$. wrapped portion of rice, serving. (5.453)

mi-dap-a $v t$. wrap up rice. (5.453)

mi-dap-sam-dap $n$. serving sized portions of rice and curry, when wrapped in leaves. (5.453)

mi-go-ra $n$. thick uncut rice plants. (5.25)

mi-ja-o, me-ja-o $n$. yesterday. (2.65)

mi-ka-sim $n$. old rice, rice cooked earlier. (5.403)

mi-kak-si $n$. leaves sticking out on top of an altar for a sacrifice to nok-ni mit-e (house spirit). (8.58)

mi-kot-chu $n$. a traditional variety of rice grown by slash and burn methods, one kind of mi-man-de. (5.25)

mi-ma $n$. big-grained variety of rice, one kind of mi-man-de. (5.25)

mi-man-de, mi-man-di $n$. hill rice, dry rice, traditional rice grown by Mandis, of which there are several types. (5.25)

mi-mang-sa $n$. agreement, reconciliation, settlement of a legal case. $<\mathrm{B}$ (8.15)

mi-mil $n$. sticky rice that has been cooked by steaming, cooked mi-mitim. (5.25)

mi-mit-im $n$. a variety of sticky rice before it has been husked, the kind of rice that, when cooked, will be called mi-mil, a traditional rice from slash and burn times, one kind of mi-man-de. (5.25)

mi-mit-im mi-rong $n$. husked but uncooked sticky rice. (5.25)

mi-mon-tron, ni-mon-tron $n$. invitation. $<\mathrm{B}(7.38)$

mi-na-tik $n$. a traditional kind of rice from slash and burn times, one kind of mi-man-de. (5.25)

mi-pal $n$. rice portion, serving, share, esp. when wrapped in a banana leaf. (5.453)

mi-pan-chi $n$. rice and curry mixed and ready to eat. (5.403)

mi-pang, mi-bi-pang $n$. rice plant, bigger than a jal-a (seedling). (5.25)

mi-pi-sa $n$. a traditional kind of rice from slash and burn times, one kind of mi-man-de. (5.25) 
mi-pil-ek, mi-bil-ek $n$. very soft boiled rice, rice cooked with lots of water, which can be eaten with gu-ru-ma native sugar or milk. (5.25)

mi-pring $n$. morning rice meal. (5.477)

mi-ra-mis $n$. non-meat foods, vegetarian foods. $<$ B (5.409)

mi-rim $n$. rice grains that have been dropped and spilled while eating. (5.403)

mi-ring $n$. echo in ha-king mi-ring property, possessions, implying the possessions of a rich person. (5.112)

mi-rong, me-rong $n$. husked but uncooked rice. (5.25)

mi-sak-a, me-sak-a vi. burn, sting, as from nettles or the stinging hair of a mang-tip worm: git-ok mi-sak-a. vi. feeling in throat after eating arum ta'-ring. (8.94)

mi-sal $n$. morning or noon rice meal. (5.477)

mi-sam $n$. rice and curry. (5.403)

mi-si, me-se $n$. mouse, rat, (see me-se for compounds). (4.419)

-mi-si- $a a$. very, a lot, much: ro'-mi-si-a very long; ak-mi-si-a plucked a lot; dal--mi-si-a very big; ring•-mi-si-a sing a lot. (3.342)

mi-si-mi, mi-si $n$. millet. (5.23)

mi-si-na-chil $n$. small leafed herb, (lit. mouse ear). (4.656)

mi-si-sam-si $n$. rice and curry, (same as mi-ja-ba, mi-sam). (5.403)

mi-sti $n$. sweets, candy. $<$ B (5.408)

mi-a, me•-a $n$. man, male. (6.821)

mi-bram $n$. edible middle part of the stalks of a banana plant. (4.670)

mi-chi gal-a $v t$. the first washing of new cloth to be rid of the starch. (6.57)

mi'-chik, me'-chik $n$. woman, adult female. (6.821)

mi-gong $n$. a long vine with $40 \mathrm{~cm}$. rather water lily like leaves with a 'v' cut at tip, (Beng. kan-chon). (4.655)

mi'-mang, me'-mang $n$. ghost. (8.54)

mi-tra, me-tra $n$. unmarried woman, young woman, adolescent girl. (6.824)

mik- cls. classifier for units of measure from the tip of the middle finger to the elbow, cubit. (1.36)

mik-a-a, mik-a-a srak-srak vi. slightly rough or uneven, not smooth or slippery. (3.662)

mik-au-a vi. wake up, open eyes. (7.97)

mik-bok-a vt. desire, covet, greedy for, lust after. (7.723)

mik-brak-mik-brak adv. seeing ahead of time, seeing early, as when someone comes from a distance. (3.512)

-mik-brang- $a a$. without seeing, fail to notice: $\mathbf{i}$-mik-brang-a go the wrong way, make a wrong turn; su-mik-brang-a stab without seeing what is stabbed; ni-mik-brang-a look past, not notice, miss seeing. $(9.538)$ 
mik-brap-a vi. be dazzled by bright light, get into the eyes, of bright lights. (3.554)

mik-brap-it-a $v t$. shine on, dazzle someone: ang-a nang-ko lait net-e mik-brap-it-a shining a light, I dazzle you. (3.554)

mik-brek, mik-brek-brek $a d v$. with eyes partly closed. (9.42)

mik-brok-a vi. dull, faded; fade, loose color, loose initial shine or glaze, grow dull. (3.539)

mik-cha-a vt. like, love, be attracted to, like with the eyes, like things, esp. like a member of the opposite sex. (7.722)

mik-chet-a, mit-chet-a, ku-mik-chet-a vi. swear, promise, take an oath. (7.33)

mik-chi $n$. tears, (lit. eye-water). (6.29)

mik-chi-ding-ding adv. stare. (9.42)

mik-chi-mrang, mik-chi-mrang-mrang adv. with running eyes. (8.93)

mik-chi-rong-rong adv. beautifully bathed, cleaned. (3.714)

mik-chil, mik-chil-chil, mik-chil-mik-chil $a d v$. very small, tiny; in a very small way: mik-chil dak-e den'-a cut into tiny pieces. (2.215)

mik-chip-a vi. close eyes. (9.42)

mik-chip-mik-kem $a d v$. embarrassed, blinking, closing and opening eyes in embarrassment. (7.745)

mik-chok-a vi. having a long face, a pointed face. mik-chok-chok $a d v$. (6.42)

mik-chret-a vi. be dazzled from brightness. (3.554)

mik-dam-bak-a vi. wide and round-faced, considered beautiful. mikdam-bak-bak $a d v$. (6.42)

mik-dat-a, sal mik-dat-a vi. dazzled with glare, blinded by getting the sun in the eyes: na-a lait net-e ang-ko mik-dat-it-a shining a light, you dazzled me. (3.554)

mik-dat-mik-dat $a d v$. staring, a bit angrily. (9.42)

mik-del-bok $n$. white of the eye. (6.22)

mik-del-bok-bok $n$. soft, of a person, of someone who doesn't want to work; lacking strength or taste, of a curry without enough soda or spices. (3.686)

mik-del-brok-brok, mik-del-bok-bok, mik-del-bu-bu adv. having big eyes, ugly-looking eyes, as when angry. (9.42)

mik-del-bu $n$. white of the eye; an insult. (6.22)

mik-del-bu-bu $a d v$. staring with wide eyes. (9.42)

mik-del-ong, mik-del-ong-rong, mik-del-rong-rong $a d v$. staring with large eyes, ugly. (9.42)

mik-gau-a, mik-gek-a vi. open eyes, as when puppies first open their eyes. (4.482)

mik-gi-sim $n$. black eye. (8.953) 
mik-gil $n$. eyelid. (6.22)

mik-gil dong-ja, mik-gil gri $v p h$. have no modesty, too forward in behavior, shameless, (lit. without eyelids). (7.745)

mik-gil jom•-a vph. sleepy, doze, (lit. heavy eyelids). (7.96)

mik-gil mik-sram $n$. eyelashes. (6.25)

mik-gil nap-a vph. get to know, become acquainted with, overcome shyness, feel free with, (lit. enter the eyelids, get into the eye). (7.745)

mik-gil rak-a, mik-gil-ak-a vi. not needing to sleep, wakeful, awake, (lit. strong eyelids). (7.97)

mik-gil si-a vi. embarrassed, with eyes down in shame, (lit. eyelids die). (7.745)

mik-gil-ak-a vi. wake up, open eyes, wakeful, be awake. (7.97)

mik-gip-bok $n$. white of the eye. (6.22)

mik-gip-eng $a d v$. looking sideways, look out of the corner of the eye. $(9.42)$

mik-git-ching-a vi. look out of the corner of the eye. (9.42)

mik-git-il-a vi. dizzy. (7.96)

mik-git-tal-a vi. awake, conscious. (7.97)

mik-jap mik-jap adv. staring in curiosity or desire. (9.42)

mik-jol-a $v i$. having a long narrow face, a pointed face. mik-jol-jol $a d v$. $(6.42)$

mik-ka $n$. rain. $(4.325)$

mik-kang $n$. face; front, front end, of a flashlight, sweater, etc.; top, of a banana leaf. next, in the future: mik-kang bil-si, mik-kang-gip-a bil-si-o next year; mik-kang ja-o, mik-kang-gip-a ja-o next month; mik-kang Ro-bi-bal next Sunday. (6.22)

mik-kang pin-ik-na kra cha-a vph. ashamed to go to someone, (lit. ashamed to show one's face). (7.745)

mik-kang-a, mik-kang-grik-a vi. face to face, face one another. (9.349) mik-kang-cha-na $a d v$. forwards. (2.14)

mik-kang-grik-e chu-a vi. sleep facing each other. (9.343)

mik-kang-o, mik-kang-cha $p p$. in front of, in the presence of, (follows genitive -ni). (1.11)

mik-kel dap-a, mik-kel-kel dap-a vi. eyes gummed up with eye dirt, eyes in bad shape. (9.42)

mik-kem-a, mik-krem-a vi. wink or blink seductively; flirt. mik-kremmik-chip $a d v$. (9.42)

mik-ki $n$. eye dirt, (lit. eye-dung). (6.29)

mik-ki-brek, mik-ki-brek-brek $a d v$. with lots of eye dirt. (6.29)

mik-kim-il $n$. eyebrow, (same as mik-sim-ang). (6.25)

mik-kol, wan-chi mik-kol $n$. old yeast used as seed to make new yeast, (same as am-bi). (5.405) 
mik-kol ting•-a vi. deep eyes, as when tired. (8.93)

mik-kong $n$. macaque, (same as ha-mak). (4.418)

mik-mak-a, mik-mak-ga-mak vi. slightly drunk, slightly dizzy, a bit confused. (5.48)

mik-mak-mik-mak, mik-met-mik-met $a d v$. poorly seen due to smoke or fog. (3.54)

mik-mal-a vi. get along well, well acquainted. (8.32)

mik-mel-a, mik-on mel-a vi. see badly, see unclearly, out of focus. (3.512)

mik-met-mik-met $a d v$. poorly seen due to smoke or fog. (3.54)

mik-mik-a, wal-mik-a vt. smoke, cause smoke to swirl around, fan or blow smoke; wave smoke about as part of sa-sat-su-a ritual; smoke out bees or animals; use smoke to blacken objects, as bamboo strips for baskets; smoke bananas to ripen them; smoke cows to be rid of mosquitoes: wal-ko mik-mik-a, mi-si ong•-kat-kan make a mouse come out with smoke. (5.422)

mik-na-geng $n$. area beside and in back of the eye, temple. (6.22)

mik-nap-a, mik-on-o mik-nap-a vi. get into the eyes, enter the eye, as dirt, dust. (9.216)

mik-neng-a vt. tired of seeing, don't want to see, dislike, (lit. eye-tired). $(7.744)$

mik-ni-a vi. greedy. (7.723)

mik-nok-sik $n$. corner of the eye. (6.22)

mik-ol-sim-sim $a d v$. eyes looking downward, in anger or in shame. (9.42) mik-ol-ting•-ting $a d v$. deep-eyed. (6.42)

mik-on $n$. eye, (A·chik: mik-ron). (6.22)

mik-on a-no $n$. pupil of the eye, black center of eye. (6.22)

mik-on an-dal-a vi. see badly, (lit. eyes dark). (3.512)

mik-on bi-rong $n$. iris of the eye, colored and dark part of the eye, (lit. eye-grain). (6.22)

mik-on gip-bok $n$. white of the eye. (6.22)

mik-on kra-a vi. become familiar with someone or something by having often seen him or it: ang-a mik-on kra-e Dhaka-cha nik-ba-jok I have come familiar with Dhaka by visiting it. (7.43)

mik-on sa-a vi. having painful inflamed eyes. (8.93)

mik-pit-i ging-pit-i $a d v$. dirty face, with nose dirt and eye dirt. (6.58)

mik-ra-chak adv. having no shame. (7.745)

mik-rak-a vi. wake up; wakeful, not need to sleep, fail to sleep at night; not shy, (lit. eyes strong). (7.97)

mik-rep-rep-a vi. blink constantly, from dirt or disease; blink seductively. (9.42)

mik-sel-a vi. wink with one eye. (9.42) 
mik-sem-pru, mik-sem-pru-pru $a d v$. without washing or bathing, with a dirty face. (6.57)

mik-sep-a, mik-sep-sep-a vi. with nearly-closed eyes. mik-sep-sep $a d v .(9.42)$

mik-si-a vi. desire, want, hope for. (7.725)

mik-si-ging-si $a d v$. lots of work, many jobs to do. (8.433)

mik-si-mik-ot $a d v$. dislike, not want to see. (7.744)

mik-sik, pu-ra mik-sik $n$. bits of rice grain that have been broken when pounded and that are given to chicks to eat. (4.677)

mik-sik-kim $n$. forehead, brows, area over the eyes. (6.22)

mik-sil-ek-a vt. lust after, be lustful. (7.723)

mik-sil-ep $a d v$. with eyes partly closed. (9.42)

mik-sim-ang $n$. eyebrow. (6.25)

mik-sim-dik-dik adv. looking down in anger or shame. (9.42)

mik-sok-a $v t$. hope, look out for, suppose, suspect, expect, (lit. eye-reach).

mik-sot-ti $n$. pimple on the eyelid, stye. (8.955)

mik-sram $n$. eyelash, very small. (6.25)

mik-srok-a vi. long-faced, thin-faced. mik-srok-srok dak-a $a d v$. (6.42)

mik-srong-a vi. long-faced, good looking. mik-srong-srong dak-a $a d v$. (6.42)

mik-su-a vi. wash face. (6.57)

mik-ta $p p$. because, on account of, for that reason, (follows genitive -ni). Echo in gi-sik mik-ta mind. (1.13)

mik-tal-a $v p h$. lie on one's back; mik-tal-e chu-a sleep on one's back. $(9.343)$

mik-tam-bik-a, mik-tam-bing-a vi. round-faced. mik-tam-bik-bik $a d v .(6.42)$

mik-ting-grik-e chu-a $v p h$. sleep with one person facing another's back. (9.343)

mik-to-a, mik-tu-a vt. measure, cloth, etc. by forearm lengths (mik). (9.66)

mik-tom- cls. classifier for units of length from the elbow to the end of the clenched fist. (1.36)

mik-u-a vi. sound, make a noise; cry of any animal, meow, bleat, moo, etc.; sound, of musical instrument, machine, etc.; squeak. (3.624)

mik-u-drak-a vi. make a loud noise, of a dog, a squeaking ox cart, an airplane. (3.624)

mik-wat-ging-wat $a d v$. looking rapidly back and forth. (9.42)

mil-a vi. fat. (6.48)

mil-dam-bu-a vi. fat, of people, cattle, etc. (6.48) 
-mil-ong dns. bare, without clothes or jewelry: ku-mil-ong-a naked above the waist; na-mil-ong without earrings; jak-mil-ong without a bracelet; ja'-chok ku-mil-ong bare legged. (6.552)

mil-am $n$. traditional ceremonial headhunting sword. (5.845)

-min-a pvs. subordinating suffix: having, being, (see -e-min-a). (0.216)

min-a vi. ripe, of crops, fruit; oozing, of a wound; cooked enough, done; turn white, of hair. (4.694)

min-chi-a vi. very ripe and soft. (4.694)

min-chu-ek-a $v i$. very ripe and soft, as bananas. (4.694)

min-da-wek-a vi. over cooked. (5.46)

min-dam-bek-a vi. over ripe, left too long before harvesting. (4.696)

min-di-ni $n$. the thatch that extends over the gable end of an old fashioned long house. (5.632)

min-ek-a vi. tender, soft, of well-cooked rice, breasts; a bit over-ripe but still edible. -min-ek- $a a$. soft, squash, crush: jot-min-ek-a poke and make soft, crush; ga'-min-ek-a step on and squash; rim•-min-ek-a squash in the hand. (3.664)

min-i-o $l w$. day before yesterday, some time ago. (2.65)

min-ik-a, min-ik-min-ik vi. level, even, smooth without lumps, as the mud of a rice field that is ready for transplanting. (3.662)

min-ku-ak-a vi. very ripe. (4.694)

min-min-ek-a vi. a bit too ripe, very soft. (4.696)

min-ok-a $v t$. swallow; eclipse of sun or moon. (5.472)

min-pret-a vi. ooze, of a sore in the ear or on the body, (lit. ripe-burst). (8.953)

min-su $n$. material that comes with a new baby and that includes blood along with the pale secretions (bi-jol), pus, bloody pus: min-su gal-a clean away the blood and dirt of a new baby. (6.77)

-min-tal- $a a$. asleep of body parts, pins and needles: ja'-min-tal-a leg is asleep; jak-min-tal-a arm is asleep. (8.94)

min-min $a d v$. very slowly, in working, in walking. (2.724)

min'-ti-sa $n$. echo in ak-ki-sa min•-ti-sa a little. (3.345)

-ming $\mathrm{cm}$. a case marker meaning with, along with, together with: angming with me. (0.12)

-ming ts. a terminal suffix that follows one of the tense-aspect markers and that indicates a conditional or a previous time. (0.312)

ming- cls. classifier for incorporeal and abstract things, diseases, songs, colors, spirits, ideas. (1.31)

ming-a $v t$. name, call, for personal names, not for family or lineage names: Ming-ji ang-ko ming-a I am named Ming-ji, (lit. Ming-ji (is the name that) names me). (7.18)

ming-kil-ak-a vt. call people by the wrong name, when joking. (7.18) 
mit-am $n$. some. (3.346)

mit-chi ga-ak-a vph. a bit of rain falls. (4.325)

-mit-chi- aa. into small pieces: den-mit-chi-a chop into small pieces; chit-mit-chi-a tear into small pieces; ak-mit-chi-a tear off into small pieces. (3.347)

mit-chi-a vt. have disgust for, be repelled by, as of bad things to eat, as Hindu feelings for lower castes. (7.744)

mit-chi-mit-chi $a d v$. a little bit: seng-na mit-chi-mit-chi a little bit light, as at early dawn. (3.345)

mit-chu-a, mit-tu-a vt. close, plug up, a bottle, bamboo tube, etc.; fill up, as stuffing mud into the cracks of drying house walls. (9.61)

mit-chu-ra $n$. cork, plug, cap, screw top of a bottle. (5.438)

mit-dap $n$. layers of a basket bottom. mit-dap- cls. classifier for stories of a multi-story building; for layers of a basket bottom: mit-dap-gin-i double bottomed. mit-dap-mit-dap $a d v$ in layers. (5.854)

mit-dil-wak $n$. jungle lizard, smaller than a map-pru, about $30 \mathrm{~cm}$. long; a fat woman. (4.441)

mit-e, mit-i $n$. spirits, gods, unseen beings that create things, cause thunder, etc., and cause disease when they bite. (8.54)

mit-il-mit-il dak-a $v p h$. shake back and forth in an earthquake. (4.37)

mit-im $n$. body fat, grease. mit-im-a vi. fat, fatty tasting. mit-immit-im dak-a $a d v$. a little fatty. (6.29)

-mit-ing-o pvs. while, at the time of: chol-a gan-mit-ing-o while wearing a shirt. (0.216)

mit-tang-a vt. care for, look after, bring up, feed, as children or old people. (9.73)

mit-tel-a int. somewhat artificial translation of English 'thank you'. (0.64)

mit-tin-a $v i$. be out of sight, hidden. (9.344)

mit-tip-a vt. close up, cover, of a bottle, a window hole, of the clouds over the sun; plug up, put on a screw top; bury; be closed, shut, without holes. (9.61)

mit-tu-a, mit-chu-a vt. plug up, close up, fill up, as mud into cracks in a wall. (9.61)

-mo ts. a question marker anticipating agreement: ga·-ak-jok-mo? (it) has fallen, hasn't it?, (A•chik). (0.31)

mo-a, mu-a vt. herd, drive cattle. (4.49)

mo-bel $n$. lubricating oil. <B (5.838)

mo-chi-chi-a, mu-chi-chi-a vt. shake, as a tree to get fruit, a person in order to awaken. (9.515)

mo-du $n$. papaya, honey. $<\mathrm{B}(5.22)$

mo-ja, mu-ja $n$. socks. $<\mathrm{B}(6.523)$ 
mo-je-jek-a vt. shake vigorously, as to loosen fruit from a tree. (9.515) mo-jim-a, mu-jim-a vi. swing, shake, move back and forth, of a pendulum, a child in a hanging cloth cradle, leaves in the wind, the earth in an earthquake, people squirming back and forth. (9.255)

mo-jim-it-a vt. move, swing, shake. (9.511)

mo-mo-a, mu-mu-a, mo-a vi. soothe, as by humming to a baby or by rocking or bouncing it gently. (7.29)

mo-ne-hoi int. I suppose so. $<\mathrm{B}(0.66)$

mo-ra $n$. a low round stool made from bamboo and cane. (5.82)

mo-rek-rek-a, mu-rek-rek-a $v t$. shake, as a tree to get fruit. (9.515)

mo-sa $n$. man of the opposite marriage group, brother-in-law, wife's mother's brother, sister's daughter's husband. (6.611)

mo-sa-ri, mu-su-ri, mu-sul, mu-sur $n$. mosquito net. $<\mathrm{B}(6.591)$

mo-si, moi-si $n$. domestic water buffalo, wild buffalo, (same as mat-ma). $<\mathrm{B}(5.35)$

mo-si-ga-ri $n$. buffalo cart. $<\mathrm{B}(5.86)$

mo-so-la $n$. spices. $<\mathrm{B}(5.405)$

mo-tor, mot-ol $n$. any kind of a motor vehicle. $<\mathrm{E}(5.86)$

mo`-na, ma'-na $q w$. why?, what for? (A•chik: mai-na). (1.21)

mo•-ong-a, mong•-a vi. ask someone to accompany, urge someone to go along, take along; urge someone to do something, attract: ang-a nang-ko mong-ing-a I am asking you to go along. (7.38)

moi-la $n$. dirt, rubbish, waste. $<\mathrm{B}(3.716)$

moi-na n. myna bird, (Beng. sa-me). $<\mathrm{B}(4.43)$

mok-at $n$. echo in chok-at mok-at door. $<\mathrm{B}(5.638)$

mol-a $n$. fragrant mixture of tobacco and molasses used for smoking with a water pipe. $<$ B (5.429)

mol-i-ka-pul $n$. white many-petaled flower. $<$ B (5.28)

mol-on-a vt. knock off the last grains from rice plants by driving cattle over them. (5.16)

mol-ong-ga $a d v$. fully naked, without clothes, like a small child. (6.552) mol-mol-a vi. request, beseech. (7.38)

mon- cls. classifier for maunds, a measure of weight, 40 seers, about 37 kilograms. $<\mathrm{B}(1.36)$

mon-dol-a vi. die down, about to die, of plants. (4.696)

mon-tro $n$. a non-Christian ceremonial chant, incantation. $<$ B (8.566)

Mong-gol-bal $n$. Tuesday. $<$ B (2.813)

mong-ma $n$. elephant. (4.419)

mong-song $n$. most important, main, primary. -mong-song- $a a$. main, primary: cha-mong-song-a eat enough; nok-mong-song main building, house of the senior couple of a household. (3.76) 
mong•-a, mo•-ong-a vt. ask someone to accompany, urge someone to go along, take along; urge someone to do something, attract: ang-a nang•-ko mong•-ing-a I am asking you to go along. (7.38)

mos-jiit, mos-jiit-nok, mo-jiit, mos-jiid, mu-jiit, mo-siit $n$. mosque. $<$ B $(5.624)$

mrak-a, mrok-a vt. wipe off, clean off, sweep off, lick off. (9.556)

-mrang, -mrang-mrang dns. resembling, somewhat: git-chak-mrang, git-chak-mrang-mrang reddish; gip-bok-mrang whitish. (3.433)

mre-o-mre-o $a d v$. faintly, dimly, not bright, of a fire: mre-o-mre-o ni-a see dimly, as a sick or dying person might see. (3.558)

mrik-a vi. smooth, as well-combed hair. mrik-mrik, mrik-dil-dil $a d v$. (3.662)

mring-a vi. knocked down, damaged, as crops from trampling animals or wind and rain. (4.696)

mrit $n$. razor, straight razor with a handle. (5.832)

mrok-a vt. wipe up, as collecting food from a dish; clean off, as a dish with ashes; clean a place for drying rice. (9.556)

mu-a $q w$. what? mu-a-ko den-ing-a? what (are you) cutting?; mu-amu-a? what all?, what? (plural). (1.21)

mu-a-hai int. what's this?, what's happening? (0.64)

mu-ri $n$. puffed rice. $<\mathrm{B}(5.403)$

mu-ri $n$. tied bundle of rice stalks. mu-ri- cls. classifier for tied bundles of rice stalks. $<\mathrm{B}(5.16)$

mu-ri ka-a vt. tie in a bundle, of rice stalks. (5.16)

mu-su-ri, mu-su-ri dail n. red dahl. (5.23)

mul-a $n$. a large radish; the plant has four-petaled white or slightly lavender flowers, and edible greens in addition to the white or reddish edible root. $<\mathrm{B}(5.27)$

mul-a, mul-a na-tok $n$. a small white fish that is without 'whiskers' (ku•-sim-ang), (Beng. dang-ki-na). <B (4.454)

mul-a sak $n$. edible radish leaves, greens of mul-a. (5.23)

-na $\mathrm{cm}$. dative case marker that marks the indirect object of a clause or sentence; to, for: ang-na to me. (0.12)

-na $\mathrm{cm}$. an augmentation for locatives indicating direction toward, and that is used with -o or -cha to give -o-na, -cha-na to toward, in the direction of. (0.12)

na int. no, not. $<$ B (1.24)

-na pvs. infinitive: $\mathbf{i}$-ang-na ha-sik-be-ing-a (I) want very much to go. $(0.216)$

-na ts. isn't it?, question particle which often anticipates a positive answer. $<$ B $(1.21)$

-na $t s$. quotative particle, it was said, someone said, I heard: sok-ba-noa-na I heard that (he) will arrive. (0.31) 
na-a vi. appear, show up, turn up, of people, fruit at the market, etc.; become visible; rise, as sun, moon; erupt, come out, swell up, of skin diseases, pimples, etc. (3.512)

na-ba-a, sal na-ba-a vi. sun rises, appears. (2.52)

-na-ba-dong-a pvs. probably. (3.42)

-na-ba-gin-ang pvs. will probably: re-ba-na-ba-gin-ang will probably come. (0.211)

na-bak $n$. collar of a shirt; loop on a basket for holding the tump line; handle of a cup; flap of rawhide to which the rope for holding a drum is attached. (5.87)

-na-be pvs. negative imperative, (A•chik). (0.215)

na-chik-grong $n$. barking deer, (same as ma-rak-ha). (4.419)

na-chil $n$. ear. (6.22)

na-chil a`-kol, na-chil a--kil-ok, na-chil-ni a-kil-ok, na-kol $n$. ear hole. (6.22)

na-chil-dang-dang, na-kil-dang-dang $n$. with big ears. (6.22)

na-chil-ja--leng $n$. earlobe. (6.22)

na-chil-ke-em-a, na-chil-ki $n$. ear wax, (lit. ear-dung). (6.29)

na-dam-bi $n$. earring worn at the top of the ear. (6.562)

na-di $n$. a stage of a maturation of a bee or other insect when the wings begin to grow but it still cannot fly; grub. (4.474)

na-dil-eng, na-ding-dil-eng $n$. ear ornament worn at the bottom of the ear. (6.562)

na-ding-di $n$. dangling ear ornament. (6.562)

na-ding-dil-ep, na-dong-dil-ep $n$. hanging lower ear ornament of present style, not a ring. (6.562)

na-dip-a, na-chil na-dip-a, na-dip dak-a vi. interrupted in hearing by noise; ring in the ears, buzz, have noise in the ears; slightly deaf, not hear well. (8.93)

na-du-ret-a vi. appear in part but not fully, of the rising sun or moon, of the sun coming from behind clouds. (3.512)

na-gan-di, to-ra na-gan-di $n$. a partially-made basket, a basket of which the cane work at the top is not yet done. (5.854)

na-gap-a vi. match, of clothes; get along, of people, (same as mel-i-a). (3.539)

na-gi-a, la-gi-a $v t$. need. $<$ B (7.726)

na-gi-ra $n$. loop on a basket for holding the carrying strap; cane handles for the sling of a rice beer pot. (5.854)

na-gok $n$. deaf. (8.92)

na-gra $n$. a type of drum with a pottery base. $<$ B (8.85)

na-grang-grang $a d v$. sticking out, of ears. (6.42)

na-gring $n$. cock's comb. (4.471) 
na-jak-a $n$. early morning, time when the sun is not quite up. (2.52)

-na-jok pvs. immediate or intentional future: kat-na-jok about to run, intends to run; kat-ja-na-jok will run no more, does not intend to run any more; ga'-ak-na-jok is about to fall. (0.211)

-na-jok-ming ts. present or future conditional or future perfect, referring to an action happening before some specified time in the future: ching-a re-ang-na-jok-ming we will have gone. (0.312)

-na-jok-o, -na-jok-o-no pvs. when about to, when intending to: nang-ni cha-na-jok-o ang-ko o-kam-bo when you intend to eat, call me. (0.218)

na-kol $n$. ear hole. (6.22)

na-kong-kep $n$. inner fold of ear that is above and behind the ear hole. $(6.22)$

na-mil-ong $n$. without earrings, without a necklace. (6.552)

na-pin-tak-tak $n$. without earrings. (6.552)

na-prot-a vi. come through, appear through. (3.512)

na-ra, na-ra-deng-ga $n$. stubble, the bottom of rice stalks left standing after harvest. $<$ B (4.670)

na-ra-ja-ting $n$. rice stubble left after stalks have been cut. (4.670)

na-rang $n$. orange tree, orange fruit. (5.22)

na-reng-reng-a $v t$. shake head. (9.41)

na-ri-kel, nai-rol $n$. coconut. $<$ B (5.22)

na-rik-ki $n$. big earrings, once worn by women, that hung in bunches from the earlobe or from a strap over the head, and that may have offered some protection against headhunters. (6.562)

na-rik-tam-bik, na-ruk-tam-buk, na-rik-tem-bik, na-ruk-tem-buk $n$. small children, used in joking. (6.822)

na-rip-pu-a vi. come out, of a rash, such as a pox or from the poisonous touch of a mang-tip worm. (8.953)

na-ron-chi $n$. a white insect that lives in dung and does not bite. (4.467)

na-rong-reng-a vi. turn head back and forth, shake the head; no. (9.41)

na-si-a vt. greedy, lust for, long for something. (7.723)

na-sik-et $n$. tick, insect that bites people and cattle, and grows round as it fills with blood. (4.468)

na-sin, ro-sun $n$. onion. $<\mathrm{B}(5.23)$

na-sin-do-ki $n$. garlic. (5.23)

na-sin-gip-bok $n$. garlic. (5.23)

na-sin-git-chak $n$. onion. (5.23)

na-sta $n$. breakfast, snack, small meal, meal without boiled rice. $<$ B (5.477)

na-tap-si $n$. an ear ornament. (6.562)

na-tek-mik-tek $a d v$. moving head from side to side, looking in all directions, of dogs, people. (9.41) 
na-teng $n$. upper side of the face, upper cheek and temple. (6.22)

na-teng git-chak $a d v$. having no shame, (lit. red cheeks). (7.745)

na-til-ek-a, na-til-ek mik-til-ek vi. restless, going this way and that; looking back and forth constantly, alert. (9.324)

na-tong $n$. the wooden stake which is embedded at the top of a mud wall and to which the roof is tied: na-tong, na-tong-ket the fulcrum around which a rice pounder swings. (5.632)

na-tong-ket $n$. cross piece of a teng-ki (rice pounder), the fulcrum around which the pounder swings. (5.87)

na-tuk $n$. very small and sacred drum. (8.85)

-na-wang $d n s$. with a big opening, a basket shape characterized by a wide mouth; with a gaping mouth, stupid. (6.42)

Na-wang $n$. a monster with a very large mouth and, some say, a large belly, who is credited with causing eclipses by swallowing the sun or the moon. Na-wang min-ok-a vph. eclipse, (lit. swallowed by Na-wang). $(8.54)$

na-wang-ku-wang adv. looking around, looking back and forth; crazy, not working, foolishly. (7.44)

na'- $c p$. prefix for varieties of fish: na'-tok fish; na'-kam dried fish. (0.17)

na'-a pro. you singular; combining form: nang-; nang-ko you accusative; nang-ni your. (1.62)

na-bak-a, ha na'-bak-a, ma'-bak-a vi. dirty, dirty from earth. (3.716)

na-cheng-bit, han'-cheng-bit $n$. kind of small edible fish, smaller than a sardine. (4.454)

na'-chi-gol-dang $n$. kind of fish, similar to sai-tan but white, (Beng. bai-la). (4.454)

na-chi-kal-tok $n$. kind of fish with white patches on its skin; a skin disease characterized by small depigmented areas. (4.454)

na-ching pro. we inclusive, you and I, you and we. (1.62)

na-dang $n$. a type of fish that is as long as an arm. (4.454)

na-gap-a vi. go together, of dialects that are similar, of matching clothes; get along well together, of friendly people. (7.11)

na'-gi-pa $n$. mother. (6.612)

na-kam $n$. dried fish of the ordinary kind. (5.401)

na'-kam wa'-sing $n$. bamboo tube used for storing dried fish. (5.438)

na-ku ja-da $n$. insult given to a stupid man, as stupid as a fish. (7.27)

na-ku-dep $n$. a kind of fish, (Beng. ma-gur). (4.454)

na--ma-su $n$. a kind of fish with a flat mouth, (Beng. ti-mi). (4.454)

na'-ong, no•-ong pro. you plural, (mainly Modhupur, same as na`-song). $(1.62)$

na-rim-il $n$. a slippery kind of fish, (same as sing-gi na-tok). (4.454)

na-rong $n$. a large kind of fish. (4.454) 
na'-si-mang pro. you plural, (A·chik). (1.62)

na'-song pro. you plural: na·-song-ni your, plural. (1.62)

na-su-deng-ga $n$. a kind of fish with a long mouth, (Beng. kai-ka). (4.454)

na-tik $n$. edible freshwater shrimp. (4.458)

na-tok $n$. fish, general term. (4.454)

na-tok-gran $n$. a kind of dried fish that Mandis prepare themselves by drying in the sun. (5.401)

-nai ts. contraction of quotative - na and emphatic -ai: do-til-eng indik-e mik-u-jok-nai it really is said that the do•tileng bird calls like this. (0.314)

nai-rol, na-ri-kel $n$. coconut. $<\mathrm{B}(5.22)$

nak-a vt. walk over stalks of cut rice in order to knock off the rice grains, by people. (5.16)

nak-da-na $n$. a very bitter herb that is eaten as cure for bad stomach and diarrhea. (8.99)

nak-pul $n$. nose jewel, ornament worn at the side of the nose. $<\mathrm{B}(6.562)$

nal $n$. barrel of a gun; pipe; tributary of a river git-ok nal gullet, (lit. neck path). <B (5.87)

nal-a vt. eat or drink a lot, gluttonously. (5.48)

nal-sa-nal-bat $a d v$. on opposite sides, across from each other, that side and this side, on both banks of a river. (2.11)

nam-a vi. good. (3.455)

nam-bru-a vi. fairly good, not very good. (3.453)

nam-chik, ang-nam-chik $n$. man's sister's daughter, woman's brother's daughter, cross-niece, niece, daughter-in-law. (6.617)

nam-e dong-a-ma? int. how are you?, are you alright? a greeting that is probably a fairly recent innovation, but one that is used more often than the other artificial translations of English courtesy forms, $(\mathrm{A} \cdot \mathrm{chik}$ : nam-eng-a-ma?). (0.64)

nam-e nik-a, nam-nik-a $v t$. like. (7.722)

nam-e-man-chan $a d v$. very good, excellent. (3.455)

nam-en $a d v$. very. (3.76)

nam-et-a $v t$. improve, make good. (3.455)

nam-gek-a vi. well off, well to do, in health or possessions. (8.91)

nam-grim-a vi. get along well. (3.455)

nam-hang, nam-sang adv. good enough, adequate, good but not excellent. most, more than half, almost, almost done. (3.453)

nam-ja vi. bad. (3.457)

nam-ja gat-a $v p h$. speak badly of, say bad things about. (7.27)

nam-kal-a vi. better. (3.455)

nam-nik-a, nam-e nik-a vt. like. (7.722) 
nam-pak-a vi. very good. (3.455)

nam-sang, nam-hang $a d v$. most, more than half, almost, almost done, good enough, adequate, good but not excellent. (3.341)

nang-a vi. produce, bear fruit, etc.: bi-te nang-ba-ing-jok the fruit has become ripe. (4.694)

nang-a $v t$. must, need, require. (7.726)

nang-a, gro nang-a vt. commit an offence, such as adultery or theft. (8.13)

nang-a, nang-ting-a, nang-ting-dat-a vt. touch, bump into something, as a tree; hit a mark, hit with something, as with something thrown. (9.552)

nang-a-nang-ja $n$. this and that, various kinds of things. (5.81)

nang-chak-a vi. make a loud noise in the ear, like a gun: chan-dal na-chil-o nang-chak-ing-a the gun is making a loud noise in (my) ear. (3.624)

nang-chap-a vi. touch, be touching, lean against. (9.552)

nang-chap-grik-a vi. touch each other, foul in football. (9.552)

nang-chek-chek-a vi. ripe, of fruit, rice, etc.; fine, rich, plentiful, well developed; stage of rice beer when it is well mixed and good to drink. (4.694)

nang-chek-chek-a, gro nang-chek-chek-a vi. a legal case, including one arising from improper behavior between a man and a women. (8.13)

nang-chrak-a vi. taste badly because of too much soda or salt. (3.684)

nang-chrap-a vi. grow in large amounts, of fruit, vegetables. (2.25)

nang-dat-a vt. bump into, collide, touch hard, press to the point of hurting, bump into with body; knock two things together. (9.552)

nang-git-ot-a $v t$. touch lightly or quickly. (9.552)

nang-gol, lang-gol $n$. plow. $<$ B (5.831)

nang-gol, rang-gol $n$. long tailed monkey, langur monkey. (4.418)

nang-it-a vt. touch, bump, hit, cause things to touch or bump. (9.552)

nang-rim-a, nang-grim-a vi. get along well, united, have a good relationship, often with implication of an illicit sexual relationship. (6.72)

nang-rim-it-a vt. reconcile, cause to get along well. (8.32)

nang-ting-a, nang-ting-dat-a $v t$. bump into, as into a tree when walking. (9.552)

nang-ting-grik-a vt. bump into each other, as when playing football. (9.552)

nang- pro. combining form of na'-a you singular. (1.62)

nang-jong $n$. your younger brother. (6.614)

nang-ma $n$. your mother. (6.612)

nang"-mong nang"-pong $n$. your parents and their kinsmen. (6.63)

nang-pa $n$. your father. (6.611) 
-nap- aa. enter, go into: chu-nap-a enter for sleeping, sleep together, a way of initiating a marriage; sik-nap-a squeeze in forcefully, as into a house or bus; kat-nap-a run in and take shelter, hide; til-nap-a fly onto a person, fly into the eye, of dust or an insect. (0.48)

nap-a vi. enter, go in, come in; set, of the sun, moon. (9.313)

nap-bol-a vi. be baptized (for Baptists). (8.564)

nap-ket-a vi. enter and stay, not come out. -nap- $a a$. enter, go into: chunap-a enter for sleeping, sleep together, a way of initiating a marriage; sik-nap-a squeeze in forcefully, as into a house or bus; kat-nap-a run in and take shelter, hide; til-nap-a fly onto a person, fly into the eye, of dust or an insect. (9.313)

nap-rong-rang-a vi. fit through, pass through freely. (2.22)

nap-sik-a, nap-sik-et-a vi. hide, in embarrassment or fear. (9.344)

-ne ts. a particle that softens an imperative or a request, and makes it more polite: ang-a nap-na-ne? may I please come in?; seng-bo-ne please wait. (0.31)

nel-a $v t$. shove, poke, with the shoulder or upper arm. (9.511)

nel-grik-a vt. strike shoulders against each other, as in bed, in scuffling. (9.552)

nem-a $v t$. look for, search. (3.514)

nem-bar, mem-bar $n$. member of the union (local district) council. $<\mathrm{E}$ (8.12)

nem-mik-brang-a vt. look past, not notice, miss seeing. (3.514)

neng-ra $n$. a lame person. (8.96)

neng-ra-bon $n$. a very fine short grass with tiny burrs. (4.652)

neng•-a $v i$. tired. -neng-. aa. tired: bil-neng-a tired; mik-neng-a don't want to see, tired of seeing; ja•-neng-a have tired legs; ku•-neng-a tired voice. (7.94)

neng-si neng•-wak adv. very tired. (7.94)

neng-sit-tap-a vi. very tired. (7.94)

neng-tak-a vi. rest, take a rest. (7.94)

neng•-wak-a vi. very tired. vi. echo in neng•-si neng•-wak-a very tired. (7.94)

net-a $v t$. turn on, shine, with a light, candle, burning sticks, etc., in order to see; shine on, illuminate: lait-cha net-e ni-ing-a, chau-kok-ko see the thief by shining a light. (3.556)

net-kri-a $v t$. clear around the edge of an area to be burned so fire will not spread beyond it; wal kam-na ken-e net-kri-a clear the edge for fear of fire burning. (5.113)

net-pak-a, net-pak-e gal-a vt. flick off, brush off with hand or broom, of dirt, unwanted food, etc.; push aside, as when people push through a crowd. (9.557) 
-ni cm. genitive case marker that marks a possessive; -'s: ang-ni my; meng-gong-ni the cat's. (0.12)

-ni $\mathrm{cm}$. an augmentation for locatives indicating direction from, and that is used with -o or bfcha to give -o-ni or -cha-ni from: $\mathbf{u}^{\bullet}$-cha-ni re-ba-a came from there. (0.12)

-ni ts. question marker that forms yes-no questions, a less common synonym of -ma. (0.31)

ni-a $v t$. look at; watch, test, try, attend to with one of the senses: kin-a-e ni-bo listen!; rim-e ni-a feel. (3.514)

ni-bru-a vi. see poorly, not want to see, not try to see. (3.514)

ni-cha-a vi. with head up, look up. (9.41)

ni-chak-a $v t$. watch for someone or something, look for someone; wait for someone, of a midwife waiting for a baby, of participants in a sacrifice waiting for the priest to return to the house. (3.514)

ni-chak-gip-a $n$. midwife, (lit. one who watches and waits for someone). (6.77)

ni-chak-su-a $v t$. wait for. (9.328)

ni-chol-wi-a $v t$. peek, sneak a look. (3.514)

ni-dat-a, ni-tat-a vt. stare at. (3.514)

ni-dat-a, sal ni-dat-a $v t$. bright sun, (lit. the sun stares). (3.556)

ni-dik-a vi. ugly to see. (3.457)

ni-gak-a vt. stare at. (3.514)

ni-git-chi-a vt. see unintentionally, catch sight of; look at something bad, as seeing someone who is very sick. (3.514)

ni-git-u-a $v i$. direct the face upward, look way up, higher than ni-cha-a. (9.41)

ni-ket-chi-a $v t$. look to one side. (3.514)

-ni-ko cm. an augmentation for locatives that is used in a clause with a transitive verb and that indicates the direction from which something comes. It is used with -o or -cha to give -o-ni-ko or -cha-ni-ko from: u'-cha-ni-ko ra'-ba brought (it) from there. (0.12)

ni-mik-brang-a vt. look around without seeing, look past, not notice, miss seeing. (3.514)

ni-mon-tron, mi-mon-tron $n$. invitation. $<\mathrm{B}(7.38)$

ni-na $\mathbf{a}^{\cdot-s i k-j a} v p h$. dislike, (lit. not want to look at). (7.744)

ni-na man·-ja $v p h$. dislike, (lit. unable to look at). (7.744)

ni-om n. law. $<$ B (8.14)

ni-on-a vi. look down, bend down to look. (9.42)

ni-pak-a $v t$. look at on the way, look at in passing. (3.514)

ni-pang-pil-a $v t$. twist around to look backwards, look over one's shoulder. (3.514)

ni-pil-a vt. look back, look over one's shoulder. (3.514) 
ni-rai-a vt. take out weeds, cultivate, esp. with a se-ni (flat bladed digging tool). <B (5.156)

ni-ri-a $v t$. quarrel, dispute, get along badly. (8.32)

ni-rik-a $v t$. watch someone leaving. (3.514)

ni-rok-a vt. observe, guard, look after, watch over, for children, animals, garden, but for a shorter time than jil-a. (9.73)

ni-rok-ki-a vt. guard, observe, care for. (9.73)

ni-rong-ret-a vi. look to the sides. (9.42)

ni-sam-a $v t$. echo in ni-rok-a ni-sam-a look after, care for. (9.73)

ni-si-a vi. echo in ni-dik-a ni-si-a ugly to look at. ni-dik ni-si, ni-dik ni-si dak-a $a d v$. in an ugly manner. (3.457)

ni-so-a, ni-su-a vt. wait for, look out for. (3.514)

ni-tat-a, ni-dat-a vt. stare at. (3.514)

ni-tat-e dong-a, sal ni-tat-e dong-a, ni-dat-e dong-a $v t$. shine on strongly, (lit. the sun stares). (3.556)

ni-tim-a vt. watch attentively, continuously. (3.514)

ni-to-a, ni-tu-a vi. beautiful to look at. (3.455)

ni-to-a cha-nap-a vi. good to look at and to eat, beautiful and tastes good. (3.455)

ni-to-et-a vt. beautify, make beautiful. (3.455)

ni-to-ri-ri $a d v$. very beautiful to see. (3.455)

ni-wat-a vt. stare at, look at nothing else. (3.514)

niil $n$. blue. $<\mathrm{B}(3.534)$

nik-a vt. see, dream about. vi. look like, have the appearance of. nik- $a a$. look upon, consider, regard: chon-nik-a feel small, feel inferior, have an inferiority complex; dal-nik-a look up to, respect, see as large; nom-nik-a appear to be easy; nam-nik-a like, love, see as good; seng-nik-a show off, act smart. (3.512)

nik-dik-a vt. ugly to look at; catch sight of, see briefly, see unexpectedly, see someone who does something bad, catch in the act, as a thief. (3.512) nik-dil-et-a $v t$. show where something can be seen. (3.516)

nik-et-a $v t$. see at a distance. (3.512)

nik-mal-a vt. see each other regularly, as of two people who work regularly together, as lovers. (3.512)

nik-man-a vi. notice, discover, notice the absence or presence of something. (3.512)

nik-rik-a $v t$. see something that is moving, see in passing. (3.512)

nim-a vt. forbid, restrict, as some kinds of food to a sick person; do without: mi-ko nim-bo, sal-ha sal-gin-i avoid rice for a day or two. $(5.479)$

ning•-a, ning•-a-o, ning•-a-cha $p p$. inside, of a house, box, mouth; under, a table, under ground, (follows nominative): ang-ni bik-ma ninga-o inside my stomach. (1.11) 
ning•-a-ni ku'-chil $n$. lower lip. (6.22)

-no $\mathrm{cm}$. locative case marker, an alternate of -o that can be used only with a limited number of pronouns and demonstratives. (0.12)

-no pvs. future, (variant of -no-a). (0.211)

no, no-gip-a, no-no, a-no $n$. younger sister, man's younger brother's wife, husband's or wife's younger brother's wife. (6.615)

-no-a, -nu-a, -no pvs. future tense: kat-no-a will run; ga'-ak-no-a will run, $(A \cdot c h i k:-g e n) .(0.211)$

no-a-bi $n$. sisters, younger and older. (6.62)

-no-a-ming, nu-a-ming $t s$. conditional, would have: ching-a $\mathbf{i}^{\bullet}$-angno-a-ming we would have gone. (0.312)

no-di $n$. river, larger than a gang. $<\mathrm{B}(4.252)$

no-gip-a, no-tang, a-no $n$. younger sister, mother's younger sister's daughter, cousin-sister. (6.615)

no-gri $n$. small metal bells tied to a pole and used by a priest during the ja-ra-gat-a (return walk from a sacrifice). (8.58)

no-gri, mat-cha no-gri $n$. plant with lovely yellow or orange, sweet pea-like flowers on 1.5 meter stalks that grows in the forest. (4.656)

no-jong $n$. younger sisters and brothers. (6.62)

no-ma $n$. female members of one's own lineage, (lit. younger sister and mother). (6.631)

no-mil, nu-mil $n$. young unmarried woman, adolescent girl, (A•chik), (same as me'-tra). (6.824)

no-mo-skar int. a Hindu greeting. $<\mathrm{B}(0.66)$

-no-na $\mathrm{cm}$. alternate of -o-na toward, used with some pronouns. (0.12)

-no-ni $\mathrm{cm}$. alternate of -o-ni from, used with some pronouns. (0.12)

no-no $n$. little sister, affectionate name for a baby girl. (6.615)

no-sa-ri $n$. wife's younger sister. (6.615)

no-sta $n$. echo in na-sta no-sta breakfast, snack. $<$ B (5.477)

no'-ong pro. you plural, (mainly Modhupur, same as na'-song); combining form: nong--. (1.62)

noi-cha, da-ba noi-cha $n$. wooden post of a water pipe. $<$ B (5.429)

noi-on-ta-ra $n$. a five-petaled white or purple flower, phlox. $<$ B (5.28)

nok $n$. house, building, household, family. -nok dns. buildings: wa'-sinok house built of split bamboo; tat-nok building where weaving is done; skul-nok school building; ran-a-nok kitchen building. nok- $c p$. prefix for types and parts of houses: nok-pan-te young men's house; nok-kap house site. nok- cls. classifier for families, households, for what is held in a house. (5.622)

nok jang-si-ni bil-bang $n p h$. crossbeam that spans the side walls of a house. (5.633)

nok-chak-a $v t$. take someone into one's home; give shelter. (9.72) 
nok-cham-e $n$. parent's of one's children-in-law, parents of a child's spouse. (6.614)

nok-chik $n$. a woman's female lineage mates, mother, sister, etc.; a man's wife's female lineage mates, wife's sisters. etc. (6.631)

nok-chol $n$. opening in the sidewall of a traditional Mandi long house, where water and objects can be thrown out. (5.638)

nok-dang, a-ba-nok-dang $n$. land and house, property of a household. (8.427)

nok-di $n$. small house for a newly married or young couple in the compound of the parents. (5.622)

nok-dong-gip-a $n$. a replacement spouse, one who marries a widow or widower; successor, esp. the successor of the a--king nok-ma (title holder of the village land). (6.657)

nok-gi-sep, nok-ni gi-sep-o $n$. space between houses. (5.644)

nok-gil $n$. back end, back door, of a traditional long house, space beside a house if there is a door on that wall. (5.638)

nok-gip-a $n$. owner, God. (8.427)

nok-jam $n$. property, (lit. house-granary). (8.423)

nok-jang-chi, nok-jang-chi-o $n$. center of the house. (5.638)

nok-ka-ni $n$. outside corner of a house. (5.636)

nok-ka-ra, nok-kra $n$. roofed area without walls at the front end of a traditional house. (5.635)

nok-kap $n$. house site, place where a house can be built. (5.644)

nok-ket-chi, nok-ket-chi-o $n$. space next to a house. (5.644)

nok-kim-a $n$. space below a house, space below anything. (5.644)

nok-king $n$. roof. (5.632)

nok-kol $n$. servant, worker. (8.41)

nok-kol gam-a $v p h$. work for wages, work as a servant. (8.435)

nok-kra, nok-ka-ra $n$. roofed area, without walls, at front end of a traditional house. (5.635)

nok-krom $n$. male heir, resident son-in-law. (6.617)

nok-ma $n$. rich person, wealthy person; traditional village leader, headman. (8.441)

nok-ma cha-a vi. be rich, not poor. (8.441)

nok-ma-gip-a $n$. owner, the employer of a worker. (8.41)

nok-man-di $n$. traditional style house, long and with the door at one end. (5.622)

nok-mik-kang $n$. front end, front entrance, of a traditional long Mandi house. (5.638)

nok-mong, nok-mong-song $n$. the main building, main house of a compound, house for the senior couple. (5.622)

nok-na $n$. heiress, daughter who inherits property and cares for her parents. (6.617) 
nok-ni gu-ang $n$. a small type of spider that lives in people's houses. $(4.464)$

nok-ni mit-e $n$. the spirit of the house. (8.54)

nok-ning $n$. interior, inside of the house. (5.644)

nok-pan-te $n$. house for adolescents and unmarried young men. (5.622)

nok-pi-song-pi $n$. whole village. (3.343)

nok-sa-gri $n$. husbands of two sisters, (same as sa-du-sa). (6.62)

nok-sam $n$. area beside a house; neighbor. (5.644)

nok-san $n$. loss, as from buying and selling. $<\mathrm{B}(8.442)$

nok-sik $n$. corner of a room, inside corner of a house. (5.636)

nok-sil $n$. neighbor, neighboring houses. (5.644)

-nol dns. place to keep animals, goat shed, place for chickens: wak-nol pig sty; do•-nol chicken coop. (0.15)

nom-a vi. easy, soft, inexpensive. (3.75)

nom•-bil-ek-a vi. cooked softly, as for children or for old people without teeth. (5.46)

nom•-brek-a vi. very soft, of mud, fruit. (3.664)

nom-chi-brek-a $v$. weak, without strength, works badly, of a person; soft, cooked with too much water, of rice. (7.56)

nom-min-ek-a vi. very soft, too soft, of mud, fruit; cooked with too much water, of rice, tubers. (3.664)

nom-nik-a $v t$. appear to be easy, appear soft. (7.43)

nom•-rik-nom•-rik $a d v$. neither very hard nor very soft, the texture of body flesh. (3.664)

nong-a vt. rub, kneed, as muscles; smear on; mix, as rice and curry when eating; grind finely. (9.554)

nong-chak-a $v t$. grind spices, etc. with grindstones: ol-di nong-chakgip-a rong-ti a stone for grinding turmeric. (5.458)

nong-rep-rep-a $v t$. roll, rub, between or on the hands. (9.645)

nong- pro. combining form of na•-ong, no•-ong you plural, (mainly Modhupur). (1.62)

not-a vt. scour, scrub, scrape, of skin, pots, etc.; clean by scrubbing, clean teeth. (9.557)

not-brak-a, not-brok-a vt. rub a part of the body against something in order to scratch it, of cows, buffalo or even people. (4.482)

not-grot-a $v t$. rub up against something in order to scratch, generally used for animals. (4.482)

-nu-a pvs. future, (variant of -no-a). (0.211)

$\mathbf{- o}, \mathbf{- u}, \mathbf{- n o}, \mathbf{- n u} \mathrm{cm}$. temporal or spatial locative case marker that marks the location of an event in time or space, in, at, on: am-bun-o tomorrow; te-bil-o on the table. -o, -u pvs. nominalizing suffix; when, at the time of: ang-ko sing-o a-gan-chak-na man'-no-a when (you) ask me, (I) will be able to answer. (0.12) 
o-a, u-a vi. open, of doors, boxes, etc. (9.211)

-o-ba pvs. although, even if: bi-a sok-ba-ja-o-ba, nik-ja-no-a even if (he) arrives, (we) will not see him. (0.216)

o-ba-ba, ba-ba, bap-a-ri-bap int. exclamation showing surprise. $<\mathrm{B}$ (0.66)

o-chol $a d v$. old and weak, lame, unable to walk. $<\mathrm{B}(6.78)$

o-chrak-a, u-chrak-a vt. peel off skin, remove peel. (5.458)

-o-de pvs. if: sok-ba-ja-o-de, nik-ja-no-a if (he) doesn't arrive, (we) will not see (him). (0.216)

o-drak-a, u-drak-a vt. peel, remove peel. (5.458)

o-et-a, u-et-a $v t$. open, cause to be open, of doors, etc. (9.60)

o-jim-a, u-jim-a vi. swing, as in a cradle. (9.255)

o-kau-a, u-kau-a vt. open, of doors; take off, of covers, bottle caps. (9.60)

o-kil-ak-a vt. open, as a box, a covered pot, door, trunk, book, skin of a banana, jackfruit. (9.60)

o-mak $n$. largish tree with lovely flowers, the bark of which is used to make tump lines, (Beng. ga-ton). (4.632)

-o-na, -no-na $\mathrm{cm}$. an augmented locative meaning toward, until, up to the time: Ep-ril-o-na until April. (0.12)

o-nek adv. many. <B (3.342)

-o-ni, -no-ni cm. an augmented locative meaning from, since: Dha-kao-ni from Dhaka. (0.12)

-o-ni-ko, -no-ni-ko cm. an augmented locative used with transitive verbs that indicates the direction from which something comes: ba-no-ni-ko ra-ba-a? from where was it brought? (0.12)

o-ro-la $n$. a tree, not found in Modhupur and known only from stories; it is said to grow wild and to bear an edible fruit. (4.632)

-o-sa, o-ha pvs. only if: bi-a sok-ba-o-sa, nik-no-a (we) will see (him) only if he arrives. (0.216)

o-suk $n$. illness, sickness. $<\mathrm{B}(8.91)$

$\mathbf{o}^{-} \mathbf{- a}, \mathbf{u} \cdot \mathbf{- a}, \mathbf{h o}-\mathbf{a}, \mathbf{h u} \cdot \mathbf{a}$ vt. clear land for slash and burn cultivation. (5.113)

o-bit-e, u'-bit-i, ho-bit-e $n$. father-in-law. (6.611)

o•-gon-a, ho•-gon-a vi. get down, get off, from a chair, bus, ox cart, train; climb down, from a tree, mountain, (same as ho•-on-a). (9.318)

ok $n$. stomach, (mainly A•chik but used in some Mandi compounds). $(6.245)$

ok man-a vi. sick to the stomach. (8.97)

ok re•-a vi. diarrhea, (A·pal). (8.97)

ok sa-a vi. have a stomach ache. (8.97)

ok-a vt. pull out, of plugs, corks, plants when weeding, etc.; unbutton; take off clothes that require insertion, of shoes, hat, shirt, lunggi; disconnect. 
-ok- $a a$. come apart, take apart, separate: sal-ok-a pull out; go-oka come apart, come loose; po-ok-a take off, take out, of hats, corks; sol-ok-a carve, as a memorial post. (9.572)

ok-bong-a $v i$. wider at the middle than at the ends, as some rice fields, some baskets; bulging section of a memorial post. ok-bong, ok-bongbong $a d v$. (2.313)

ok-chok-a vi. bulging, of a distorted circle. (2.313)

ok-dip-ra $n$. soft area under the sides of the ribs, further front than ok-sam-beng. (6.245)

ok-gam-a vt. call, name, (A•chik, A·pal). (7.18)

ok-gil-a vi. noise made by a bull when wanting to lock horns. (4.476)

ok-gin-ang-a vi. pregnant. (6.74)

ok-ka-a $v t$. satisfied by, eaten enough of, full of, drunk from. (5.48)

ok-ka-bing-bing adv. ate lots, full. (5.48)

ok-ka-mik-mak-a vt. partly, but not completely full, of food, rice beer; slightly drunk. (5.48)

ok-ke, da'-o-ni ok-ke $a d v$. not for long. (2.53)

ok-ki-sa, ok-ki, ok-ki-ok-ki $n$. a little, a small amount. (3.345)

ok-kri-a vt. hungry, hungry for. (5.48)

ok-ma-ru-ru $a d v$. big-bellied, of people, animals. (6.48)

ok-o ki-a vi. be pregnant, (lit. hang in the belly). (6.74)

ok-pel-pel $a d v$. fat, of people. (6.48)

ok-rip-pong-a vt. pull away, pluck, of harvesting cotton. (5.16)

Ok-ro-hai-on, Ok-ran, O-gron, Ok-rai-on $n$. a Bengali month that overlaps November and December. $<$ B (2.828)

ok-sam-beng $n$. soft area at the side of the body below the ribs. (6.245)

ok-sik-ki $n$. soft area at the side of the body below the ribs. (6.245)

ol-a, hol-a vt. carry or hold from tump line; wear na-rik-i (bunch of large earrings) as protection against head hunting. (9.532)

ol-chon-a vi. small, of a bunch of bananas. (2.211)

ol-dal-a vi. big, of a bunch of bananas. (2.211)

ol-di, el-di, hol-di, hel-di $n$. turmeric. $<$ B (5.405)

ol-ko-pi, ul-ko-pi $n$. a member of the cabbage family with a bulbous, edible stalk. (5.23)

ol-u-kok $n$. thief. (8.34)

on-a vi. come down, of rain, fog, dew: ku·-chi on-a drool: on-et-a vt. put down, set down, as a load from the head, shoulders, etc.; put over one's head, of an umbrella. -on- aa. down, downward: chok-on-a get down, get off, as from a bus; ni-on-a look down; sik-on-a push down; bil-on-a fly down; di-on-a take a pot off the fire; sik-on-a push down. (9.68) 
on-dap-a $v t$. cover with something; put the new, horizontal strip on top of the old, vertical strip in basket-making; put on top, of rows of thatch. (9.62)

on-dim-a, hon-dim-a vi. short, low, near the ground, of a house, tree, post, hill; opposite of chu-a-a, chu-ak-a tall. (2.11)

on-drak-a, on-drak-drak-a vi. rain heavily. (4.327)

on-jet-a, on-jet-dap-a vi. put something heavy on top of something else. $(9.67)$

on-krop-a, on-krop-dap-a vt. drop onto. (9.68)

on-prak-prak-a, on-drak-pak-a vi. rain heavily. (4.327)

on-sit-tu-a $v t$. set down firmly. (9.68)

on-wak-a, gu-ri on-wak-a vi. be thick, of fog. (4.329)

on-a, hon·-a, ron-a vt. give; put; apply, as paint; pay a fine, compensation; give a loan; officiate at a marriage; take an examination (what students do), hand in an examination, (see ron- for compounds). (9.72)

on-cheng-a $v t$. loan an object that must be returned. (8.446)

on-chok-a vi. climb down headfirst, like a woodpecker; put down point first. (9.318)

on'-dim on'-chok $a d v$. up and down, of roads, of birds flying, of people of different heights walking in a file; uneven, of ground. (2.14)

on-dim-it-a vt. make lower, make shorter, of a post. (9.649)

on-grip-a vi. covered, as with clouds, cloudy: ga-dil-a on·-grip-e donga cloudy. (9.62)

on-kam-a vt. give finally, not to take back. (9.72)

on-ki-rot-a $v t$. give a little but not all, as money. (9.72)

ong $n$. a large stinging hornet that builds a very large nest with an opening at the bottom. (4.463)

-ong ns. plural marker that indicates a person along with his, her, or their closest people, usually their family members: Ra-ben-ong Raben and his family. (0.11)

ong-ga-ri $n$. the shelf over an old style central fireplace. (5.636)

ong-a vi. be, exist, be equivalent to. (9.11)

ong-ja-o-ba conj. or, if not. (1.4)

ong-kat-a, hong-kat-a vi. come out, appear, emerge; come out, of skin diseases. (9.313)

ong-kat-dil-a vt. cause to come out, make something come out, squeeze out. (9.572)

ong-mi-sep-a vi. seep out, come out of the ground in small amounts, of water in a spring. (9.279)

ong•-on-a vi. get down, get off, from a chair, bus, ox cart, train; climb down, from a tree, mountain, (same as ho-on-a). (9.318)

ong:-pa-a vi. be together. (9.11) 
ong--pok-pok-a vi. come out, appear in large amounts, of water from a spring, rice and other grains when maturing. (9.279)

-ot- $a a$. call attention to, pay attention: jak-si-ot-a point to, show; sim-il-ot-a smell, test by smelling; dong-ot-a touch with a finger to call attention; ni-ot-a watch, look at. (7.47)

-ot- $a a$. let go of, separate from: rong-ot-a take out something round, as a banana from its skin; sil-ot-a push out, as the hand out of a sleeve; rim-sik-ok-a let something escape from one's hand, as a bird one is trying to catch. (9.54)

ot-a, hot-a $v t$. sweep, rake, clean up. ha-ba ot-a $v p h$. rake up debris in fields. (9.58)

ot-e gal-a $v t$. sweep and throw away, clean out and throw away: na-chilki·-ko ot-e gal-a clean out and get rid of earwax. (9.58)

ot-rok-a $v t$. sweep. (9.58)

ot-tong-ga $n$. earring worn at top of ear. (6.562)

-pa dns. male human: dal-am-pa a man with a high opinion of himself; rak-am-pa a very strong man; ra-song-pa a proud man. (6.821)

pa-a, pa-gip-a $n$. father. (6.611)

pa-chat-chi $n$. father's kinsmen. (6.63)

pa-dal, pa-dar $n$. Catholic father, priest. $<\mathrm{E}(8.51)$

pa-dri $n$. Anglican priest. $<\mathrm{E}(8.51)$

pa-gar, pa-gal $n$. small tank, pond; hole from which dirt has been taken and that collects water. $<$ B $(4.253)$

pa-gip-a-na-gip-a $n$. parents. (6.62)

pa-gol, pa-gil-a $n$. crazy person, one who speaks incoherently. $<\mathrm{B}(8.92)$

pa-gu-ri, pa-gri $n$. turban. $<\mathrm{B}(6.522)$

pa-jong $n$. father's older brother, mother's older sister's husband. (6.611)

pa-jun $n$. stick for hitting a cow, walking stick; the vertical bamboo next to the jang-ki (stairs) of an altar. $<\mathrm{B}(5.833)$

pa-ki $n$. thirty decimals of land, three-tenths of an acre. $<$ B (5.12)

pa-lok $n$. Protestant preacher, (same as pa-mong). (8.51)

pa-mong $n$. Protestant preacher. (8.51)

pa-ra $n$. open earthenware dish, pottery holder for coals that give smoke in sa-sat su-a ceremony. (5.432)

pa-ra $n$. section of a village, neighborhood. $<$ B (8.22)

pa-ra-pi-sa $n$. small earthenware dish. (5.432)

pa-ri $n$. wound, sore, infected sore. (8.955)

pa-sa, ging-ni pa-sa $n$. round ear ornament inserted in the nose, not a ring. $<\mathrm{B}(6.562)$

pa-sim-bi-dil $n$. thin cultivated vine with little clumps of leaves along it that can be cooked with meat. (5.23)

pa-ti, am-pa-ti $n$. fine type of mat, the kind used to sleep on. (5.852) 
pa-wa-sa, pa-ning-sa $n$. father and child. (6.62)

pa-wang-a vi. be open, of an umbrella. (9.211)

-pa- $a a$. with, along with: don-pa-a put together; a-gan-pa·-a speak along with; $\mathbf{i}$-ang-pa'-a go together, go with. (0.41)

pa·-a vi. brave, fearless. (7.746)

pa·-a vt. apply, smear on, as paint, as medicine on skin; attach, as a poster on a wall. (9.556)

pa·-a, ba・-a vi. perch, of birds; cling, as flies as on a wound. (4.482)

pa·-a, ba・-a vi. thin. (2.215)

pa-dit-a vt. come very close, esp. to something hot. (9.552)

pa-jret-a vt. press on, as medicinal herbs on a sore spot; push down, as a button switch on a flashlight. (9.67)

pa'-sik-kap-a vi. stick, be stuck to, as a poster on a wall; well dressed, with well-matching clothes. (9.63)

pa-tap-a vt. glue to, stick to, attach. (9.63)

pai-ka-na $n$. latrine. $<\mathrm{B}(5.622)$

pait class. classifier for layers of mud on a new house, (same as tol-op). $<\mathrm{B}(5.638)$

-pak dns. area of body where the limbs meet the torso: jak-pak inside of the upper arm; si-pak inside of the upper part of a woman's leg; ripak the inside of the upper part of a man's leg; gang-pak the part of a bird's wing that is close to the body. pak- $c p$. prefix for the area of the shoulder and armpit: pak-krong shoulder; pak-wal armpit; paksim-ang underarm hair; pak-sik-kep-a hold or carry under the arm. (6.233)

-pak- $a a$. along the way, while going, along with: bre-pak-a buy along the way; i-ba-pak-a go by way of; ring-pak-a drink along the way; dong-pak-a stop along the way; sap-pak-a swoop down and take, like a hawk. (0.43)

pak- cls. classifier for halves, half an areca nut, half a fruit, half lengthwise pieces of bamboo; sal pak-sa half a day; le-ka pak-sa one side of a piece of paper; the other side: ra-ma pak-sa the other side of the road. (1.37)

pak-a vt. empty out, pour out quickly, of either solids or liquids; dump. $(9.276)$

pak-a vt. shove, poke, esp. with the shoulder or upper arm: pak-grik-a strike shoulders and upper arms against each other. -pak- aa. shove, bump, push aside: jit-pak-a shove something to make it move; sik-paka push a short distance, knock over; ga-ping-pak-a push with the foot, kick away; net-pak-a flick off, brush off, with hand or broom; dok-pak-a knock off, as fruit from a tree; han--pak-a abort spontaneously, miscarry. (9.511)

pak-grik-a vt. strike shoulders and upper arms against each other. (9.552) 
pak-kim-il, pak-wal-kim-il $n$. underarm hair. (6.25)

pak-kram $n$. tree with a pale trunk. (4.632)

pak-krang chuk-krang $a d v$. speak loudly, angrily, rapidly: pak-krang chuk-krang dak-e bol-a kin-a-to-ja speaking in a loud and angry way sounds unpleasant. (7.15)

pak-krong $n$. shoulder. (6.233)

pak-ma $n$. most, more than half, majority: pak-ma-in pin-jok most of it is thatched. (3.342)

pak-ma-ri $n$. butterfly. (4.469)

pak-nok $n$. cook house, kitchen. (5.622)

-pak-pak- aa. a lot, fast: wa-pak-pak-a rain heavily; ki-pak-pak-a defecate a lot. (3.342)

pak-pak-a vt. wave hand up and down; grang pak-pak-a flap wings quickly, as when preparing to take off; flap ears, of a dog; slap the upper arm against the side. (4.486)

pak-ram $n$. place where rong-chu (flattened rice) is poured in the rongchu gal-a ceremony; place where something is poured; place where waste is thrown. (8.58)

pak-ri $n$. horizontal house beam running lengthwise, at the level of the eaves or higher, and supporting the roof. (5.633)

pak-ri-ni krong $n p h$. post in a house supporting the beam called pak-ri. (5.633)

pak-ru-ru-a vt. pour out, empty out. (9.276)

pak-sa $n$. the other side, of a road, etc.; half: sal pak-sa half a day.] C99:63, ba-tor pak-sa-cha, on the other side of the ba-tor(dike). $137 \mathrm{dem} \cdot-$ [cls classifier for folds. (1.37)

pak-sa-cha, pak-sa-cha-pak, pak-sa-o $l w$. beyond, on the other side of: ra-ma pak-sa-cha on the other side of the road. (2.11)

pak-sam- cls. classifier for sides, as sides of a sheet of paper, of a coin. (1.37)

pak-sik-kep-a $v t$. hold or carry under the arm. (9.532)

pak-sim-ang $n$. underarm hair. (6.25)

pak-tang- cls. classifier for sides, of a coin, a sheet of paper, (same as pak-sam). (1.37)

pak-wal n. arm pit. (6.233)

pal $n$. the metal front part of a plow; blade. (5.87)

pal $p p$. because, on account of, (follows genitive -ni). (1.13)

pal- cls. classifier for leaf bundles, portions of rice or curry held in a banana leaf. (1.34)

pal-a $n$. haystack, straw stack, a carefully stacked pile of rice straw, not just a heap. (4.66)

pal-a vt. sell. (8.444) 
pal-ding-a $v t$. peddle, sell by walking around with one's goods; prostitute oneself. (8.444)

Pal-gun $n$. a Bengali month that overlaps February and March. $<$ B (2.832)

pal-ong-sak $n$. a small, $15 \mathrm{~cm}$. diameter vegetable that has odd white and brown flowers on a stalk, and with star-like spreading edible leaves. $<\mathrm{B}(5.23)$

pal-rik-a vt. sell to one who is passing. (8.444)

pal-sit-ong-a $v t$. sell everything. (8.444)

pal-wang-bi-du $n$. a rather tree like vine, with straight, but not standing, sections, and fern-like leaves and leaflets. (4.655)

pam n. pump. $<\mathrm{E}(5.838)$

pan-chi-ni $n$. a ceremony before a wedding, when pan (betel leaf) and chi-ni (sugar) are offered to guests. $<\mathrm{B}(8.566)$

pan-te, pan-ti $n$. bachelor, unmarried youth, adolescent boy. (6.824)

pang- cls. classifier for trees, stalks, individual trunks of a banana plant, plants, rice seedlings. (1.32)

pang-dal-a vi. big, of trees. (2.211)

pang-kim-pang-kam $a d v$. messy, esp. of uncombed hair, beard, but also for things in the house. (3.718)

pang-na $a d v$. always, $(\mathrm{A} \cdot$ chik $) .(2.71)$

pang-na-ba $a d v$. never, when used with a negative verb, (A•chik). (2.71)

pang-pik-a vi. come out by the roots, fall over so roots come out. (9.212)

pang-pil-a vi. reversed, backward, upside down, of car, boat, basket, batteries; inside out; fall over, of a person: ang-a ring-ko pang-pil-ita I turned the boat upside down. -pang-pil- $a a$. reversed, backward: jak-pang-pil-a with hand held behind the back; ni-pang-pil-a look backward, over one's shoulder; di-pang-pil-ek-a upside down. (9.214)

pang-pil-chu-pil $a d v$. reversed. (9.214)

pang-pil-ek-a vi. turned over. (9.214)

pang-pil-ek-chu-pil-ek adv. upset, reversed, capsized; said to be the way Mandis speak Bengali. (9.214)

pang-pil-tai--a vi. turn over. (9.214)

pang-si-dim-dim $n$. lacking trees or bamboo, without growing things, empty of plants or buildings, bare. (4.630)

pang-si-ri-ri $a d v$. beautiful, the way the world looks in the bright moonlight. (3.554)

pang-si-ru-ru, pang-si-rim-rim $n$. cleared, especially of trees and brush, clear and nice. (4.630)

pas-tor $n$. Protestant preacher. $<\mathrm{E}(8.51)$

pat $n$. jute, burlap, jute thread. $<$ B (5.29)

pat-a $v t$. dress another person; esp. dress someone in jewelry; tie together cattle for a yoke; ku-tip pat-a bestow a turban as a symbol of office, 
create a headman, (lit. clothe with the turban). pat- cls. classifier for things made of cloth, shirts, lunggies, quilts; for units of paper money, takas. (6.53)

pat-chon-a vi. narrow, of cloth pieces. (2.211)

pat-dal-a vi. large, wide, of cloth. (2.211)

pat-kel $n$. brick, dried mud brick. (5.636)

pat-sa, dang-ga pat-sa $n$. one taka. (8.447)

pat-ta-ba-hor, pat-ta-ba-kor $n$. two kinds ornamental plants, one with yellow speckled leaves, one with yellow or red striped leaves. $<\mathrm{B}(5.28)$

pe, pi, pe-top, pi-top $n$. cheek. (6.22)

pe-bok-a, pi-bok-a vi. round-cheeked, puffy-cheeked. pe-bok-bok, pi-bok-bok $a d v$. (6.42)

pe-bu-su, pi-bu-su $n$. disease with swollen sides of face. (8.956)

pe-cha, pe-sa $n$. owl. $<\mathrm{B}(4.43)$

pe-chu, pi-chu $n$. bird's crop. (4.471)

pe-del $n$. pedal. $<\mathrm{E}(5.872)$

pe-pe $n$. papaya. $<\mathrm{B}(5.22)$

pe-ti-kot $n$. woman's undergarment. $<\mathrm{E}(6.526)$

pe-a, pi-a $v t$. break, take apart; hatch; change money; clear or cultivate a dry field with bamboo knife, hoe, or plow; construct a new rice field; grind flour at a mill; gin cotton; draw water from a deep well, use a water pump with an up and down handle. (9.284)

pe`-a, $\mathbf{p i}^{\bullet}-\mathbf{a} v t$. call by name: bi-ming $\mathbf{p i}^{\mathbf{i}-\mathbf{e}}$ ring-gam-a call by name. $(7.18)$

pe'-dem-a, pi'-dem-a vt. fold, bend sharply, of harder things than dem·-a. $(9.641)$

pe-e a-gan-a, pi•-e a-gan-a $v t$. explain, break down the topic and explain. (7.34)

pe'-ga-gal-a, pi'-ga-gal-a vt. break and throw away. (9.284)

pe-gom-a, pe-gop-a vt. break and bend over but not break apart, of plants. (9.284)

pe-prak-a, pi-prak-a $v t$. break completely. (9.284)

pe-prak-e a-gan-a, pi-prak-e a-gan-a vi. explain, speak fully, break it down completely and explain. (7.34)

pe-top rip-om-a, pi-top rip-om-a $v p h$. swollen cheeks. (8.956)

pe-wak-a, pi-wak-a vt. break down, cause to collapse, destroy. (9.284) pek- cls. classifiers for packs of cigarettes. $<\mathrm{E}$ (1.34)

pek-a vt. intoxicated from, surfeited from; the feeling of having eating or drinking a lot, feeling after eating pork fat: ang-a wak-mit-im-ko bang-e cha-e pek-a having eaten a lot of pork fat, I am surfeited. (5.48)

pek-chrap $n$. growing close together, parallel, as tree trunks or branches. (4.671) 
pek-drak-a vi. spread apart: ja-chok pek-drak-a with legs spread apart. (9.349)

pel-a vi. flat. pel- $c l s$. classifier for flat things such as boards and things made from boards, furniture; for flat pieces of firewood. (2.311)

pel-chep-chep adv. flat. (2.311)

pel-dal-a vi. wide, of flat things, boards, firewood, chests, hips, stools: pel-dal-dal $a d v$. (2.211)

pel-dang-dang $a d v$. wide, of flat things, of hips. (2.211)

pel-deng-a vi. flat. adv.: pel-deng-deng, pel-deng-pel-deng. (2.311)

pel-dep-a $v i$. flat. pel-dep-dep, pel-dep-pel-dep adv. (2.311)

pen-ta-long $n$. long pants. $<\mathrm{E}(6.527)$

peng-reng-peng-cheng $a d v$. rocking back and forth, like a boat or a flimsy bridge. (9.218)

peng--a vt. block off, hinder, prevent, stop, protect, block from view. peng- $a a$. obstruct: chang-peng-a blocked off; dong-peng-a obstruct; chu-peng-a prevent, obstruct. (9.77)

peng-chak-a $v t$. defend someone else against attack. (8.36)

peng-rik-a vt. hold back, prevent from going, hold in fish by blocking a stream. (9.77)

pet-ta-rong, pet-ta-rong-rong, pet-rong-rong adv. naked above the waist; hairless, either naturally or shaved. (6.552)

pet-tang- cls. classifier for buttocks. (1.37)

pi-ka cha $n$. tea served without milk. $<$ B (5.409)

pi-pi $n$. whistle, a child's toy. (8.85)

pi-prot, pi-prot-ki-sang $n$. common forest-living bird, bigger than a du-ma-sek-i, colored white, and black or gray, with red rear. (4.43)

pi-prot-ki-sang $n$. tree with red, somewhat sumac-like fruit in the cold season; named for the bird of the same name. (4.632)

pi-prot-mak-bil $n$. big variety of pi-prot bird that lives in the forest. (4.43)

pi-ra $n$. edge of the earth platform of a building. (5.637)

pi-ta $n$. fried cakes eaten at celebrations. $<$ B (5.409)

pi-tol $n$. brass, used for very good kitchen ware. $<$ B (5.88)

pi-a, pe-a vt. break, take apart; hatch; make change (money); clear or cultivate a dry field; construct a new rice field; grind, flower at a mill; gin cotton, (see pe-a for compounds). (9.284)

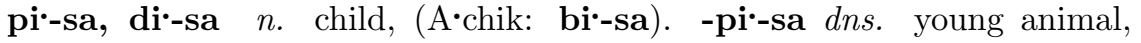
occasionally used for young of plants; a small example of objects: mong-

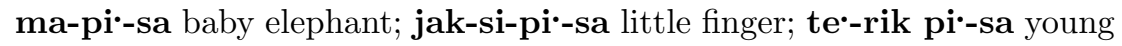
banana plant; profit, interest on a loan. (6.822)

pik-a vt. pull out by the roots, as plants from the earth, as rice seedlings for transplanting; pluck, as gray hairs, as bird down. (9.572) 
pik-chrak-a vt. pull out vigorously, as plants by roots with stones, dirt, etc., perhaps with some noise. (5.16)

pil- cls. classifier for coins. (1.38)

pil-ak $n$. all, whole, $(\mathrm{A} \cdot$ chik $)$. (3.343)

pil-dal-a vi. big, of coins, earrings. (2.211)

pil-eng-pil-eng, pil-eng-pil-ung $a d v$. rock back and forth, of a boat; flap back and forth, of paper, etc. (9.218)

pil-ip-pil-ap nik-rik-a $a d v$. catch a glimpse of. (3.512)

pil-ip-pil-ip, pil-ip-pil-ip dak-a adv. flutter strongly in the wind, of cloth, of feathers on a bird. (9.216)

pil-a vi. return, go back, come back; transform, as from human to a tiger and back again. -pil-- aa. return, reverse, again: $\mathbf{i} \cdot$-ba-pil-a come back, return; pang-pil-a reversed, upside down; am•-pil-a turn away, turn around; wal-pil-a come back, return; tang-pil-a come to life again; gi-sik ra-pil-a change one's mind. (9.315)

pil-tai-a vi. repeat, again and again. pil--tai-pil-tai $a d v .(2.71)$

pin-a $v t$. cover with, spread over, as with a blanket, thatch on a roof: bi-a lep-ko pin-e chu-a he sleeps with a blanket spread over (him). (9.62)

pin-bru-a vt. cover too much, as of hair covering the face, a blanket hiding the head. (9.62)

pin-dap-a $v t$. cover, roof with thatch, sun by clouds, bed with a blanket; smooth over furrows of a plowed field: sal-ko ga-dil-a pin-dap-ing-a the clouds cover the sun. (9.62)

pin-dim-a $v t$. spread over two or more things or people: ba-ra patsa-ko man-di ak-gin-i pin-dim-e chu-a two people sleep under one blanket. (9.62)

pin-ek-a vt. crush. -pin-ek- $a a$. crush: cha-pin-ek-a chew into pieces; ga-pin-ek-a knock down (plants) by walking on them; rim--pin-ek-a crush in the hand; jom-pin-ek-a sick all the time, crushed by disease. (9.578)

pin-grim-a $v t$. be covered together, when two or more things or people are covered by the same object; cover together when several people work together to cover something. (9.62)

pin-ik-a, pin-ik-et-a $v t$. show, point to, point out. (3.516)

pin-ji-ra $n$. cage. $<\mathrm{B}(5.852)$

pin-kin-ap-a vt. spread comfortably, as a blanket. (9.62)

ping-a, bik-ma ping-a vi. swollen, bloated, of the abdomen in disease. (8.956)

ping-chri-ping-chri $a d v$. rapidly, of running, jumping, swimming, riding a bike, etc. (2.726)

ping-gi-ra $n$. a black-colored bird that is the size of a dove, (drongo). $(4.43)$ 
ping-pang, pi-ring-pang $n$. morning star, (A`chik: pring-prang). (4.212)

pit-a vt. split, chop firewood; split bamboo; cut into small pieces. (9.573) -pit-chi pvs. only, just. (3.344)

pit-chi-ri-a, pit-si-ri-a vt. split into small pieces, esp. bamboo. (9.573) pit-drak-a vt. split, as firewood, as a length of bamboo. (9.573)

pit-ket-chi-a $v t$. split off a thin strip of bamboo with a cut perpendicular to the surface. (9.573)

pit-ku-rak-a, pit-ku-rang-a vt. split lengthwise into pieces with an axe; split bamboo. (9.573)

pit-man-di-a vt. split off a thin strip of bamboo with a cut parallel to surface. (9.573)

pit-nek $n$. any of various impure, often muddy colors: off-white, tan, gray, brown, dirty pink, dirty yellow, lightly colored, faded. (3.539)

pit-si-ri-a, pit-chi-ri-a vt. split into small pieces, esp. bamboo. (9.573)

po-da-neng $n$. finger-sized insect that makes noise during the daytime in April or May. (4.469)

po-ki $n$. bird. $<\mathrm{B}(4.43)$

po-liis $n$. police. $<\mathrm{E}(8.12)$

po-liis, nel po-liis $n$. nail polish. $<\mathrm{E}(6.565)$

po-ra $n$. reading, lesson. $<\mathrm{B}(7.28)$

po-rai-a vt. teach: pi-sa-ko ma-star po-rai-a the master teaches the children. $<\mathrm{B}(8.6)$

po-re, po-re-po-re, por, por-por, po-ro $p p$. after, (follows genitive -ni): i-ni po-re after this. $<\mathrm{B}(1.12)$

po-res-tar $n$. forester. $<\mathrm{E}(8.41)$

po-ri-a $v t$. read, study. $<\mathrm{B}(7.28)$

po-ri-bar $n$. family. $<\mathrm{B}(6.657)$

po-ri-dil-a $v t$. help to read, teach to read. (7.28)

po-ri-ka $n$. test, examination. $<\mathrm{B}(8.6)$

po-ri-skar, po-ri-skal vi. clean. $<\mathrm{B}(3.714)$

po-sak, poi-sak, po-sak-po-ri-chot $n$. clothing, dress, garments. $<\mathrm{B}$ $(6.521)$

po•-ok-a vi. come off, of a hat; come out, as a cork. (9.572)

poi- cls. classifier used in chants at sacrifices. (1.38)

poi-la $a d v$. first. $<\mathrm{B}(3.3801)$

poi-sa $n$. coins, small change. $<\mathrm{B}(8.447)$

pok $n$. fork, part of a bicycle. $<\mathrm{E}(5.872)$

pok-pek-a vi. slosh back and forth, of water in a pot when being carried, of people riding a bus; make jerking movements with the head, as when a pig eats. (9.218)

pol-pol-pal-pal $a d v$. speaking quickly and loudly. (7.14) 
pong $n$. ladle made from a gourd and used esp. for rice beer. (5.438)

pong- cls. classifier for long cylindrical things, biris, cigarettes, memorial posts, blowing horns (which have a long cylindrical mouthpiece), injections (because they are administered with a cylindrical needle). -pong dns. cylindrical: bi-pong handle of a tool; jak-pong upper arm. (1.32) pong-dal-a vi. big, of cylindrical things, gourd dippers, cigarettes. (2.211) pong-si $n$. device made from a thread hanging from a horizontal bamboo bow, used in the kil-ding tet-a ceremony for identifying the spirit responsible for a person's illness. (8.58)

pong-sreng-sreng $a d v$. long of cylindrical things. (2.312)

pos-chiim, pot-chiim $n$. west. $<$ B (4.218)

pot-a vt. accuse; threaten, make angry, make claim on, often in a legal sense. (7.36)

pot-a vt. tuck in, as a shirttail or the back part of a loincloth; insert a feather in the hair or turban. (6.53)

pra-pra $a d v$. in great numbers, of birds, ants, people. (3.342)

pra-to-na $n$. prayer. $<\mathrm{B}(8.564)$

prai $a d v$. almost, mostly; approximately. $<\mathrm{B}(3.348)$

-prak num. every, each: sop-ta-prak every week; bil-si-prak every year. (2.71)

-prak- $a a$. a lot, strongly, completely: ru-prak-a pour lots of water; bi-prak-a break completely; gil-prak-a peeling, of skin; a-gan-prak-a explain, say fully; kam-prak-a burn strongly. (3.72)

prak-a vt. break, tear open, split apart, break into pieces. (9.284)

prak-e-pruk-e $a d v$. in any old way, carelessly, without thinking, esp. of speaking: prak-e-pruk-e in-ing-a speak without holding back, speak out in any way, speak carelessly. (7.14)

prak-prak $a d v$. continuously, freely, openly, without hesitation, without shyness or fear. prak-prak mik-u-ing-a vph. noisy, loud, as the noise of water flowing on steep ground, crackling noise of splitting wood, of tearing cloth. (2.71)

pran-a vi. brave, able to, manage, reach. (7.746)

prap $n$. banyan tree, (Beng. bot). (4.632)

prem $n$. frame of a bicycle. $<\mathrm{E}(5.872)$

pret-a vt. break apart; split bamboo more coarsely than chit-a; cause something to burst open, as a bamboo wall, bamboo tube; break up, as a mud wall. -pret- $a a$. cause to explode, burst, make noise, break apart: dok-pret-a hit and break something, as a coconut; gam-pret-a make a lot of noise, like a gun, airplane; ga'-pret-a burst or squash something with the foot; kim-pret-a thunder; chrik•-pret-a scream loudly, squeal, of pigs. (9.284)

pri-pri $a d v$. into pieces: -pri-, -pri-pri- $a a$. into pieces: ak-pri-a tear into pieces; go-pri-a scatter; den`-pri-a, den-pri-pri-a cut into many 
small pieces; git-chit-pri-pri-a full of tears; cham-pri-a worn out and in pieces. (3.347)

pring, pring-o $n$. morning, in the morning, (A•chik). (2.52)

pring wal-ni $n$. tomorrow morning. (2.52)

pro-tom, pol-tom $n$. first, beginning. $<\mathrm{B}(3.3801)$

prok $n$. dress, frock. $<\mathrm{E}(6.526)$

prong-a vi. to fly about in the air, of light things such as dust, powder, cotton fluff. -prong- $a a$. swirling about in the air: cho-prong-a winnow; dok-prong-a brush off, as dirt from one's arm; man·-prong-a blow around, of dust, fluff, etc.; sip-prong-a blow off dust, dirt. (9.216) prot-chu-chu $a d v$. high and fat, of a straw stack, of a pile of things: bik-ma prot-chu-chu having a very big and round belly. (2.319)

pru-a vt. pierce, as a wound to get out puss or to work out a sliver. -pruaa. through, cut through, pierce: rat-pru-a cut through; ga'-pru-a step through; dok-pru-a knock a hole through; su'-pru-a pierce, as a wound to get out a thorn. (9.571)

pu-a vt. make a hole, as with a chisel; drill or eat holes in wood, fruit, etc., by worms or birds; plant with a digging stick. (9.571)

pu-a, pu-gek-a vt. add dry ingredients to food when cooking, of salt, soda, etc., pour, let fall from the hand, of powder or fine things like rice grains, salt into a curry; sprinkle over, as yeast over rice when making rice beer. (5.46)

pu-ju-a $n$. lay out, a sick person on a mat, a baby in a hanging cradle; esp. lay out and prepare a dead body. (6.79)

pu-kur, pus-kin-i $n$. large tank or pond that holds water all year without drying up, bigger than a pa-gar. $<\mathrm{B}(4.253)$

pu-pa-pu-pa $a d v$. with lots of holes, as a screen. (2.38)

pu-ra $n$. flour, rice flour, made by soaking rice in cold water and then pounding in a teng-ki (rice pounder); food made from rice flour; powder. (5.403)

pu-ta- cls. classifier for drops; spots, as spots of color on a lunggi; forehead cosmetic spots. $<$ B (1.37)

pu-ta-pu-ta $a d v$. in drops, spotted, like a leopard. (3.538)

pu-ti $n$. echo in na-ti pu-ti grandchild. $<$ B (0.5)

pui-ta, pu-ti-a n. rabbit, (same as kor-gus). $<\mathrm{B}$ (4.419)

pul $n$. flower; diamond-shaped 'flower' pattern of basket weaving. $<\mathrm{B}$ (4.676)

pul, kul n. masonry, brick, or iron bridge for people and vehicles. $<\mathrm{B}$ (5.646)

pul-ko-pi $n$. cauliflower. $<$ B (5.23)

pul-pen $n$. long pants. $<\mathrm{E}(6.527)$

pup, pur-bo n. east. $<$ B (4.218) 
Pus $n$. a Bengali month that overlaps December and January. $<$ B (2.829) pus-kin-i, pu-kur $n$. large tank or pond that holds water all year without drying up, bigger than a pa-gar. $<\mathrm{B}(4.253)$

put- cls. classifier for feet, 12 inches. $<\mathrm{E}(1.36)$

put-ka mach $n$. a fish that puffs up if you blow on it. Children make a ball from it and play with it. $<$ B (4.454)

ra- vt. cut by slicing, used in compounds only, (same as rat-, which see for compounds). (9.573)

ra-ja-gu-ru, ra-ja-gu-ru men-da $n$. a vegetable with tiny green-pink lantern-shaped flowers on a stalk that gets smaller toward the top, and 5 cm. leaves. A kind of men-da. (5.23)

ra-ja-has $n$. goose. $<\mathrm{B}(5.35)$

ra-ma $n$. road, path, way, means. (5.646)

ra-ma a'-sol-sol $n p h$. downward slope of a road. (4.232)

ra-ma song-a, ra-ma song-cheng-a vi. make the first inquiries about a marriage proposal, (lit. cook the path, prepare the path). (6.651)

-ra-ra ns. lots, all over the place, all around: nok-ra-ra houses everywhere; ka-rang-ra-ra dirt everywhere, filthy; kim-il-ra-ra hairy, lots of hair. (0.11)

ra-si-ra-wak dak-a $v p h$. act with modesty: ra-si-ra-wak dak-ja show off. (7.745)

ra-sin-a, sin-a vt. to cut across evenly, at a neat right angle, especially a length of bamboo. (5.856)

ra-song $n$. praise, pride, good fortune, glory; ra-song dak-a give honor with clasped hands. (7.748)

ra-song-si-si dak-a vph. boastful, show off, lacking modesty. (7.745)

ra-sot-a vt. kill by cutting, slicing, as one kills a chicken. (6.79)

ra'-a vt. bring, get, take, receive objects: gro ra'-a receive a fine, get compensation; jin-ang ra-a take a loan, receive a loan, borrow; po-rika ra'-a give an examination, what teachers do, receive the exam paper. (9.519)

ra-a $v t$. belong to a kin group such as as Cham-bu-gong or Sang-ma): ang-a Ma-rak-ko ra'-a I belong to Marak. (6.657)

ra'-ang-a vt. take, take away. (9.519)

ra-ba-a $v t$. bring, bring in this direction. (9.519)

ra-ba-gal-gal-a $v t$. bring quickly. (9.519)

ra-bit-a vt. bring along, take along, of objects in one's hands, as an animal takes something in its mouth. (9.519)

ra'-bru-a $v t$. follow without belief, as religious rules. (8.564)

ra'-chak-a vt. accept, receive objects; catch a thrown object; obey, follow orders or instructions, take advice. (9.531)

ra-chak-grik-e a-gan-a vph. answer back and forth, dialogue. (7.32) 
ra-chap-a vt. put or take along, take one thing with another thing. $(9.519)$

ra-du-a vt. lift up, take up, bring up. (9.532)

ra'-e adv. including, having to do with, taking into account, on account of that: ang-ko ra-e kam rim-bo include me in your work. (3.346)

ra-gat-a, de ra·-gat-a vt. adopt a child, esp. a sister's daughter, so that in the absence of a true daughter she can care for her adopted parents and inherit their property. (6.657)

ra-jring-a vt. keep taking, continuously taking. (9.519)

ra-kang-a, a'-kang-a $v t$. have a claim on a kinship group for a spouse. (6.651)

ra-kol-kat-a $n$. a knobly trunked tree with edible fruit that is ripe in July. (4.632)

ra-nap-a vt. bring in, take in, as into a house. (9.519)

ra'-rik-a vt. keep for a while; care for temporarily, as when one gives food to an adult; obtain from a passing peddler. (9.531)

ra-ron-a $v t$. take down, put down, unload; separate; reject, as to reject someone who is not of the right kin group. (9.511)

ra-sek-a $v t$. take by force, remove a thing or a person by force, as when a family takes back a member from an unsuitable marriage. (9.519)

rai-song-ma, ra-song-ma $n$. a proud woman, boastful woman, show-off. (7.748)

rai-song-pa, ra-song-pa $n$. a proud man, a boastful man, show-off. $(7.748)$

rak-a vi. hard, strong, tough, difficult: dam rak-a expensive. rak-erak-e $a d v$. fast, strong, of talking, walking, etc. -rak- $a a$. strong, fast: gong-rak-a fast; mik-rak-a wake up, strong in the eyes; mang-rak-a healthy, strong of body; ding-rak-a strong of long slender things, like string; jak-rak-a quick, fast, esp. in working with one's hands, quick at eating. (3.664)

rak-a-si $n$. shooting star, name of a mit-e (spirit). (4.212)

rak-am-pa $n$. a very strong man. (7.56)

rak-dam-dam $a d v$. sitting firm, not movable. (3.75)

rak-e-rak-e $a d v$. fast, strong, of talking, walking, etc. (2.726)

rak-ki-a vt. keep, maintain; guard, take care of, as a small baby or animals. (9.73)

rak-nik-a $v t$. regard as hard, difficult; appear to be difficult. (7.43)

rak-rak-a vi. echo in tak-tak-a rak-rak-a make a tapping sound, as the sound of beating a finger on the table, of typing. (0.5)

rak-rak-a vt. swing knees or feet back and forth; wag, of a dog's tail: ja'-chok-ko rak-rak-a swing the feet ; ja'-git-teng-ko rak-rak-a swing the knees, legs. (9.47) 
rak-sot-sot $a d v$. strong in language, not accepting what another person says. (7.56)

-ram dns. place, location: chu-ram sleeping place; gop-ram grave, burying place; at-chi-ram birthplace. (0.16)

ram-a vt. put out to dry in the sun. (9.272)

ram-ram $a d v$. ordinary, normal, usual. (3.44)

ran-a vi. growl, roar, of a dog, tiger, etc.; purr, of a cat. (4.476)

ran-a, ran-a-nok $n$. kitchen building. $<$ B (5.622)

ran-da $n$. widower. (6.64)

ran-di $n$. widow. (6.64)

ran-a vi. dry out, become dry, of objects, hair, etc.; git-ok ran`-a vi. thirsty, (lit. throat dry). (9.272)

ran'-chok-a vi. thin, of people. scrawny, (lit. dry pointed). (6.46)

ran-sik-ap-a vi. dry out, of the throat from talking, of a wound. (9.272)

rang $n$. gong, a traditional musical instrument. (8.85)

-rang ns. plural marker: man-de-rang people; nok-rang houses. $(0.11)$

rang-bet-ul $n$. a kind of rang (gong). (8.85)

rang-dil $n$. a kind of rang. (8.85)

rang-gol, ha-mak rang-gol, nang-gol $n$. long tailed monkey, langur monkey, hanuman monkey. (4.418)

rang-i, rang-ri $n$. largish tree with willow-like leaves and somewhat spotted bark, (Beng. ga-ton). (4.632)

rang-kong-si $n$. a kind of rang. (8.85)

rang-mi-sil $n$. a kind of rang. (8.85)

rang-sal-git-chak $n$. kind of red ant that is larger than smil-chak, (same as breng-gu. (4.465)

rang-sip-pok $n$. part of a memorial post; a kind of rang (gong). (8.58)

rang-tal $n$. plate, dish. (5.434)

rang-- cls. classifier for number of times. (1.31)

rang-du-a, rang-du-rang-du dak-a vi. breathe deeply, breath heavily. (9.45)

rang-kan-ta $n$. someone who gets angry easily. (7.766)

rang-kap-kap adv. flat, hollow, of a belly, very thin, (opposite of rongdal-a). (6.46)

rang-ki-rang-ki, rang-ki-rang-dat $a d v$. speak loudly and angrily. (7.766)

rang•-ra-a vi. belch. (9.44)

rang-si-rang-dang $a d v$. speak angrily, in an ugly way. (7.766)

rang-sing do-a vi. echo in bi-ka so-a rang*-sing do-a. (7.766)

rang-sip-it-a vi. breathe: rang-sip-it-gi-ja hold one's breath. (9.45)

rang-sok-a vi. get one's breath, able to breathe: pin-bru-o rang-sokja (I) can't breathe when covered. (9.45) 
rang•-tang-tang, rang•-tang-rang•-tang adv. angrily; hard, well inflated, of a football or tire. (7.766)

ras-ta $n$. road, smaller than a so-rok. $<\mathrm{B}(5.646)$

rat-a vt. cut by slicing; cut with sickle as one harvests rice; cut off mud from a house wall to smooth it. (9.573)

rat-a, wal-e rat-a vt. make fire by using friction. (5.424)

rat-gro-a, rat-gru-a vt. cut lengthwise. (9.573)

rat-kim-bak-a, ra-kim-bak-a vt. cut part way through. (9.573)

rat-ku-rak-a adv. cut once lengthwise. (9.573)

rat-pru-a, ra-pru-a vt. cut a hole through. (9.573)

rat-si-ri-a, ra-si-ri-a vt. cut into small pieces, as pork, pineapple. (9.573)

rat-srik-a, ra-srik-a vt. make a careful slicing cut. (9.573)

rat-tim-bang-a, ra-tim-bang-a $a d v$. cut in half crosswise. (9.573)

rat-tong-a, ra-tong-a vt. cut crosswise into two separate pieces. (9.573)

rat-wak-a, ra-wak-a vt. cut off, slice off, as a piece of meat. (9.573)

rat-wil-a, ra-wil-a, rat-wil-wil-a vt. cut around, as when skinning an eel, when cutting a stripe around a handle; girdle a tree. (9.573)

rau, lau $n$. gourd, a long edible squash that hangs from a climbing vine. $<$ B (5.23)

rau'-rau-tau-tau $a d v$. speaking loudly and rapidly: rau'-rau-tau-tau dak-e bol-a kin-a-to-ja it sounds badly to speak loudly and rapidly. (7.14)

re, ri $n$. cane. (4.655)

re-ding, ri-ding, re-ding-ga, re-ding-ra $n$. horizontal pole hung inside the house to hold clothes. (5.82)

re-du-ra, ri-du-ra $n$. kind of cane used in basket making. (4.655)

re-jap $n$. reserved forest, reserved seat on a bus. $<\mathrm{E}(4.630)$

re-jil-a vi. joke, tease, especially among some kinsmen, as between brothers-in-law, or with a grandchild. (7.26)

re-kot $n$. land records. $<\mathrm{E}(5.12)$

re-ma, ri-ma $n$. a large type of cane. (4.655)

re-man-di, ri-man-di $n$. variety of cane used in basket making. (4.655)

re-wa-a, re-wak-a vi. soiled, spotted, dirty, from ink, mud, dust, etc. (3.716)

re-wa-se-wa $a d v$. spoiled, filthy from dung, mud, etc. (3.716)

re•-a, ri·-a vi. go, come. (9.312)

re-am-a, i-am-a vi. wander, roam, take a walk. (9.319)

re•-ang-a vi. go, move away. (9.312)

re-ang-gip-a $n$. previous, last, former: re-ang-gip-a bil-si last year; re-ang-gip-a Ro-bi-bar-o last Sunday. (2.62)

re-ba-a, ri-ba-a vi. come. (9.312)

re-ba-gip-a $n$. next, the one that comes: re-ba-gip-a ja-o next month; re-ba-gip-a Ro-bi-bar-o next Sunday. (2.63) 
re•-el-a $v t$. fail to obey, defy, not follow instructions: re•-el-e i'-ang-a go, in spite of instructions. (7.33)

re-el-a $v t$. make a hole, bore a hole. (2.38)

re-em-re'-em $a d v$. having a small pattern, as the creases in one's skin. $(3.538)$

re-jring-a vi. come regularly, often. (9.312)

re-king, e--king, he-king, ri--king $n$. woman's wrap-around skirt. (6.526)

re-ping-grik-a vi. pass, of two people going in opposite directions. $(9.317)$

re'-rik-a vi. follow. (9.328)

re'-ru-ra'-a, re'-ru-rat-a vi. go back and forth, travel back and forth, of people. (9.315)

re'-sal, he'-sal, e'-sal, $\mathbf{i}^{\bullet}$-sal $n$. banana leaf. (4.675)

re-sek-a vt. pass, of one person by another when going in the same direction, overtake. (9.317)

re-tong-tang-a vi. take a short cut. (9.319)

rek sal-a $v t$. made a line, a row, as when plowing or when planting. (5.14)

rek-ka $n$. paper; notebook. $<$ B (5.88)

rek-keng-a, rik-keng-e cha-a vt. eat bones and all. (5.479)

rek-rek-a vt. move back and forth; wag tail, swish tail like a cat getting ready to pounce; shake, as a fruit tree to knock down fruit: bal-wa bolbi-jak-ko rek-rek-a the wind shakes down the leaves. (9.515)

rek-tek $a d v$. completely; often with negative as none at all: rek-tek dil-jok utterly poor; mi-rong rek-tek ong-ku-ja-no-a there will not yet be any rice at all. (3.343)

rem-bu-a, rem-bu-bu-a vt. tickle; shake someone to call attention. $(9.515)$

rem-rem-a vi. feel sleepy; blinking in sleepiness; with drooping eyelids. (7.96)

ren-da-mok-a vi. lazy. (7.94)

ren-di-a vi. dried out and in poor condition for eating, of fruit and vegetables. (4.696)

ren-jar $n$. kind of forest officer, ranger. $<\mathrm{E}(8.41)$

reng-gun-mi $n$. a variety of rice that is no longer used. (5.25)

reng-reng-a vi. ring, as a gong rings. (3.624)

reng-rim-it $a d v$. yellowish, yellow mixed with other colors. (3.532)

reng-si $n$. needle; string or stick on which to hang flowers or other things; string of flowers, fruit, fish, etc. (6.598)

reng-si $n$. a ground-dwelling worm that eats cloth, earth-needle, (same as sil-eng-si). (4.467)

reng-si a-kil-ok $n$. eye of a needle. (6.598) 
reng-si-a vi. at the ga-stu stage of early maturation. (4.692) reng-ti, leng-ti $n$. old fashioned man's loincloth. $<\mathrm{B}(6.527)$

rep-a $n$. half-taka coin, eight anna coin. (8.447)

rep-a vt. cut finely, chop up fine, make fine cuts from the surface, cut off, as skin from fruit; carve off surface, peel; echo in sol-a rep-a carve off surface. (9.573)

rep-chok-a, den-chok-a, rat-chok-a vt. carve a point. (9.573)

rep-pi-a vt. smear mud on a wall, plaster with mud. (9.556)

rep-rep-tep-tep $a d v$. quickly, thoughtlessly, loudly, as when arguing. (7.14)

rep-rok-a vt. carve off bits from the surface of something, as from a potato or tuber, so as to cook it. (9.573)

rep-sem-sem-a vt. cut into very small pieces, crush. (9.578)

ri, re $n$. cane, (see re- for compounds). (4.655)

ri- $c p$. prefix for male genitals and their parts: ri-gong penis; ri-spil testicles. (0.17)

ri-bo $n$. a lone male not attached to a group, as a-mak ri-bo lone macaque, (lit. swollen genitals). (4.40)

ri-bo na-a, ri-bo nang-a $v p h$. swollen testicles. (8.956)

ri-dam-bu-bu $n$. insult given to a man, (lit. big penis). (7.27)

ri-gong $n$. penis. (6.249)

ri-gong sil-a $v t$. retract the foreskin. (6.249)

ri-jang $n$. testicles. ri-jang bong-got-bong-got $n p h$. a way of joking about a little boy's testicles. (6.249)

ri-jang-jot-jot $n$. insult for men. (7.27)

ri-ka ka-rok $n$. insult for a man with no hair. (7.27)

ri-ku-chil $n$. foreskin, (lit: penis-lip). (6.249)

ri-met $n$. semen. (6.29)

ri-mik-del-bu $n$. insult to a man. (7.27)

ri-na-wang $n$. insult given to men, (lit. penis-wide mouthed). (7.27)

ri-pak $n$. inside of the upper part of a man's leg. (6.249)

-ri-ri dns. beautiful, well done: ni-to-ri-ri beautiful to see; pang•-si-riri beautiful, the way the world looks in the bright moonlight; ra-ja-ri-ri well done; chu-ak-ri-ri very high. (3.455)

ri-ri-si-ri, ri-ri-si-si $a d v$. messy, in a very bad state: ri-ri-si-ri dil-a very poor. (3.457)

ri-sim-ang $n$. male pubic hair. (6.25)

ri-sip-il, ri-sip-il bi-gron, ri-sip-il bit-chi $n$. testicle. (6.249)

ri-sip-il gin-ang $n$. uncastrated, (lit. with testicles). (4.49)

ri-su-brok $n$. insult given to men, (lit. penis-rotten). (7.27)

ri-ti ni-ti $n$. law, custom. $<\mathrm{B}(8.14)$

$\mathbf{r i}^{\bullet}-\mathbf{a}$, re•-a, $\mathbf{i}^{\bullet}-\mathbf{a}$ vi. go, come, move, (see re•- and $\mathbf{i} \cdot$ - for compounds). (9.312) 
ri-rau-a vi. flash, of a mirror or flashes of lightning. ri॰-rau-ri-rau $a d v$. (4.344)

ri-rau-ri-rau $a d v$. flashing, of lightning, of reflections from a tin roof, the surface of water, a TV screen. ri-rau-a vi. (3.556)

riik-sa $n$. rickshaw. $<\mathrm{B}(5.86)$

riik-sa-wal-a $n$. rickshaw peddler. $<\mathrm{B}(8.41)$

riin, run, lun $n$. loan of money, as from a savings club. riin ra-a $v p h$. take a loan, receive a loan. $<\mathrm{B}(8.446)$

-rik- $a a$. follow, along the way: kin-a-rik-a listen to something passing; ja-rik-a follow; hai-rik-a recognize; dong-rik-a stay behind; gi-sik ra-rik-a remember; nik-rik-a see in passing, see something moving; a-gan-rik-a speak to someone who is passing; man-rik-a recover something lost; rim·-rik-a hold someone back, prevent from going; dong-rika pregnant. (9.328)

rik-a vt. build, esp. for houses. (5.631)

rik-a vt. spin thread, etc. (6.598)

rik-a, rik-et-a vt. drive away, chase away animals. (4.49)

rik-a-a vt. pile up, collect in one place, as in a basket; take something from one place to another. (9.535)

rik-ang-a vi. go to inform, deliver a message. (7.34)

rik-bok $n$. old fashioned full necklace of long white cylindrical pieces, said to have been worn by both men and women. (6.563)

rik-en-rik-en $a d v$. uneven, pitted, wrinkles of the skin; a zigzag pattern. (3.662)

rik-i $n$. top, tree top. (2.11)

rik-i ben-chot $n$. top end, very top. (2.11)

rik-ing $n$. edge, border, of a piece of cloth, stripe along the border; back, non-cutting edge of a cutting tool such as an at-te (bamboo knife); top rim of a basket; edge, lip of the mouth of a pot. (5.87)

rik-kak-rik-kak $a d v$. bony, having no flesh on one's bones. (6.44)

rik-kap-rik-kap $a d v$. not smooth, bumpy, rough, of boards, cloth. (3.662)

rik-ki-jem--jem $n$. this and that. (5.81)

rik-ki-kong-si $n$. this and that. (5.81)

rik-ki-rik-kot $a d v$. rough, uneven, of boards, roads, the ground, food containing hard bits. (3.662)

rik-king-rik-king $a d v$. lumpy, as a field after plowing; scattered, disorderly. (3.718)

rik-kit $n$. each, every: man-de rik-kit every person. -rik-kit num. each, every (when used with a classifier): man-de sak-rik-kit each person; a-ba dam-rik-kit each field; sal-a-rik-kit each day; ja-rik-kit each month. (3.35)

rik-kit-a, rik-kot-a $v t$. scrape out, scrape off hard material when cleaning a pot. (9.557) 
rik-kit-dang-kit, rik-kit-rik-kit $a d v$. everything, this and that, all sorts of things. (5.81)

rik-kit-rik-kit, rik-kit-dang-kit $a d v$. everything, this and that, all sorts of things. (3.343)

rik-kot-rik-kot $a d v$. wrinkled, of skin, ground. (2.319)

rik-mat-chu $n$. oldfashioned full necklace, similar to rik-bok but with red cylindrical pieces rather than white. (6.563)

rik-ong $n$. edge, border; border between nations; bank, of a river or tank, etc.; border of cloth; edge of a bed, mat, well. (2.11)

rik-pa-a vi. follow after. (9.328)

rik-pak-a $v t$. drive away animals, pigs, birds, cattle. (4.49)

rik-pil-deng, pil-deng $n$. oldfashioned coin necklace. (6.563)

rik-rak-a vi. reflect, shine, of a mirror, tin roof, metal, water, TV. rikrak-rik-rak $a d v$. (3.556)

-rik-rik- $a a$. repeatedly, piece by piece, constantly, progressively: am•-rikrik-a want continuously; bu-rik-rik-a lie constantly; gu-ri-rik-rik-a turn again and again; bang-rik-rik-a more and more, increase, of prices; dil-rik-rik-a grow poorer and poorer; do-rik-rik-a go up and up. (2.71)

rik-rik-rak-rak, rik-rak-ga-rak, rik-rik-sik-sik, sik-sik-sak-sak, siksik-gil-ak, sik-sak-gil-ak, sik-sak-ga-sak $a d v$. restless, wiggly, esp. of wiggly restless children; moving back and forth, wandering around. (9.324)

rik-rok-a vt. scrape together, scrape into a pile. (9.535)

rik-ru-ru-a $v t$. drive away, drive animals far away. (4.49)

rik-si-kok-si $n$. this and that. (5.81)

rik-u ni-a $v p h$. watch from behind, watch as a person or animal goes away. (9.328)

rim-a vt. take, bring, for people. (9.519)

rim-a-rim-a, rim-ang-rim-ang $a d v$. see or hear partially, unclear, blurred, as from far away. (3.54)

rim-bit-a vt. bring, take along, as a child, friend, or dog. (9.519)

rim-chap-a $v t$. go together, take someone along. (9.519)

rim-il-a vi. slippery, smooth. (3.662)

rim-il-tet-tet $a d v$. very smooth, worn smooth, like a fine mat, smooth metal; shiny, like silk. (3.662)

rim-it, rim-it-a vi. yellow; by extension: light brown, orange-brown, green, occasionally blue. (3.532)

rim-it-jrang-jrang vi. completely yellow. (3.532)

rim-it-jri-jri adv. bright yellow. (3.532)

rim-it-rim-it dak-a $a d v$. yellowish. (3.532)

rim-ol $n$. pestle, pounding pole of an old style rice pounder; the piece of a teng-ki (foot treadle rice ponder) that pounds into the hole; rim-ol, 
rong-te rim-ol the smaller, upper, grindstone that is held in the hand. (5.87)

rim-pak-a $v t$. meet someone on the way and take him along. (9.519)

rim-pi-rim-pi $a d v$. very high, very far. (2.11)

rim-pit-rim-pit $a d v$. see poorly because something is far away. (3.54)

rim-ra-rim-ra, rim-ra-ga-ra $a d v$. vaguely, blurry, unclear, see badly, as from far away, in a fog, under water. (3.54)

rim-rik-a $v t$. hold someone back, prevent from going. (9.531)

rim-rim-tim-tim $a d v$. lacking strength, tired, unable to work because of pregnancy. (7.94)

rim-a vt. catch, capture, grab, hold, touch, handle; work; lead a legal case; follow the law. (9.531)

rim•-a, gam rim•-a vph. work, do labor. (8.433)

rim-brek-a vt. grab with the hands, as when eating. (9.535)

rim-brin-a vt. mix with the hand, as rice and curry. (9.535)

rim-brok-a $v t$. spoil by grabbing, as with dirty hands. (9.531)

rim-brot-a, rim-brot-brot-a vt. kneed, of sore muscles; rub, as when washing vegetables. (9.554)

rim--chak-a $v t$. catch a thrown object; receive a newly born baby. (9.531) rim-chak-gip-a $n$. one who receives, esp. a midwife. (6.77)

rim•-chak-ra $n$. something one grabs and holds regularly, something for holding, handle, handle of a pump. (9.531)

rim-chet-chet-a $v t$. grab hard. (9.531)

rim-chip-a, rim-chip-et-a vt. grab or catch something that is not seen, as catching fish in a trap. (9.531)

rim-dang-a $v p h$. finish working, without interruption. often negative: rim•-dang-ja disturbed, interrupted. (8.433)

rim-deng-deng-a $v t$. hold lightly pinched between the fingers. (9.531)

rim-dep-a vt. push with a finger or thumb, to kneed sore muscles, to test the ripeness of fruit. (9.554)

rim•-e ni-a $v t$. feel, test by feeling. (3.64)

rim-ek-a vt. touch with the palm of the hand, handle. (9.552)

rim•-ga-gal-a $v t$. work quickly. (8.433)

rim-gek-a, rim-gek-gek-a vt. grab and hold with force, grab by the throat; feeling in the throat that comes from eating arum (ta-ring), a tuber. (9.531)

rim-jang-jot-a vt. grab in the middle or narrow part, squeeze in the middle, grab by the wrist. (9.531)

rim--jep-a vi. cover over with the hand. (9.46)

rim-jret-a vt. squeeze hard with the hand. (9.531)

rim--jrot-a, rim·-jrot-jrot-a, rim•-jot-a $v t$. grab and squeeze hard. $(9.531)$ 
rim-kam-a vt. keep working, not rest. (8.433)

rim-kin-ap-a vi. pleasant to touch, feel soft or good. (3.64)

rim-krep-a vt. grab and crush. (9.578)

rim-min-ek-a vt. squash in the hand. (9.531)

rim•-pi-jot-jot $v t$. grab hard. (9.531)

rim-pi-jrot-a, rim--pi-jot-a vt. grab hard, as a stick, as someone's throat. (9.531)

rim-pin-ek-a vt. crush and mix with hand, as mud and water. (9.578)

rim-pret-a vt. break open, break with hands. (9.284)

rim-rik-a vt. hold back, not let go, not allow to escape. (9.531)

rim-rok-a vt. rub, rub along, rub with the palm, massage, kneed sore muscles, soothe by stroking; rub in order to clean; rub someone's arm in requesting, beseeching; pet an animal; brush off dirt from body. (9.554)

rim-sik-kap-a, jak-o rim•-sik-kap-a vt. hold tight in the hands. (9.531) rim-sik-ok-a, rim•-sik-ot-a vt. let something escape from one's hand, such as a bird one is trying to catch. (9.54)

rim-sot-a vi. grab by the throat, kill by choking; feeling in the throat after eating arum (ta-ring), a tuber. (3.682)

rim•-srap-a vt. manage to catch, as a chicken or a thrown object. (9.531)

rim-sret-a vt. let slip from the hand; rub off with the hand, stroke off.

rim•-srot-a $v t$. not succeed in grabbing, let slip from the hand. (9.54)

rim-su-su-a vt. do only part, fail to finish. (9.14)

rim-tat-a vt. grab by force, as when capturing a son-in-law. (6.651)

rim-tip-a $v t$. cover with the hands, esp. to cover one's mouth in embarrassment; cover the nose against a smell; cover a hole, a pot, glass, etc. $(9.62)$

rim-to-a vi. nice feeling, feel good to the touch. (3.64)

rin-ek-rin-ek, rin-ek-a-rin-ek-a $a d v$. condition of someone who is sick and weak and cannot get up; knocked down, smashed down, as rice plants that are knocked over. (8.93)

rin-ok-a vi. not too hard and not too soft, of cooked rice; not too wet and not too dry; speaking slowly and with fine language; smooth. rinok-rin-ok $a d v$. (3.664)

ring $n$. boat. (5.86)

-ring- $a a$. at the correct time: sok-ring-a arrive at the right time; most often used in the negative; sok-ba-ring-ja not arrive on time; re-angring-ja not go on time. (2.51)

ring-a vt. drink; take medicine orally, both liquids and pills; smoke tobacco. (5.474)

ring-chak-a vi. fit in, hold, of a drink: ring-chak-gip-a gil-as glass that holds the drink. (5.474) 
ring-chot-a, ring-chot-chot-a vt. slurp, as tea; suck, as on a straw, drink up; finish drinking with a slurp, as through a straw. (5.474)

ring-dam-bu-a vt. drink a lot. (5.474)

ring-drak-a $v t$. drink a lot at one time. (5.474)

ring-e ni-a $v t$. taste a liquid, test by tasting. (3.682)

ring-gam-a vt. call, call out, cry out to someone, call together, call by personal name. (7.18)

ring-gek-a $v t$. while drinking, in the midst of drinking. (5.474)

ring-gil-a vt. root up ground with the snout, like a pig; move food around with the nose, like a cow. (4.487)

ring-kong $n$. dish or hollowed log for feeding animals, especially for pigs, feeding trough. (5.835)

ring-pak-a vt. drink along the way. (5.474)

ring-pek-a vi. somewhat drunk from rice beer; choke on water, as when swimming or drinking. (5.48)

-ring-rang- $a a$. back and forth: kat-ring-rang-a run back and forth; go-ring-rang-a throw back and forth. (9.515)

ring-rang-ga-rang $a d v$. constantly moving; flicker, of reflections in metal, a TV screen, water; shaky, wobbly, of a rocking boat, a bamboo bridge; flutter, of leaves ; restless, constantly moving, of people. (9.324)

ring-reng-a vi. swing, rock back and forth, like a boat or a bamboo bridge; wiggle back and forth, like a weak chair. ring-reng-ring-reng, ring-reng-ga-reng $a d v .:$ ang-a do-ri-ko ring-reng-ga-reng ka-dingde-a I tie up the string swingingly. (9.218)

ring-ri-a vi. hang loosely, dangle, as fruit that is about to fall, like a broken branch, an injured arm. ring-ri-ring-ri $a d v$. (9.255)

ring-srip-a, ring-sip-a vt. slurp, as when drinking hot tea from a plate, hot soup from a spoon; drink through a straw; drink part but leave part, what a mosquito does when it bites. (5.474)

ring-a $n$. sing. (8.83)

ring-dim-dim-a vt. sing a lot, continuously. (8.83)

ring-drak-a $v t$. sing loudly. (8.83)

ring-mi-si-a $v t$. sing a lot, for a long time. (8.83)

ring-rang-a vi. small amount of lightning. (4.344)

ring-ring-a-ri grap-a vi. a high moaning cry. (7.29)

rip-a vi. dive under water, sink, disappear under water, flood, rise, of river water. $v t$. rub on, apply to the head, as soap, water: sko rip-a wash hair. (9.279)

rip-ak-a vt. rub or smear on, of powder, nail polish, medicine on skin; rub oil on gongs to make them look fine. (9.556)

rip-ak-rip-ak $a d v$. quickly, all at once, of collecting things, of using ones hands: rip-ak-rip-ak, rip-ak-sam-bak speaking quickly. (2.726) 
rip-ak-tip-tip-a $v t$. spread on plentifully, as oil on the body, powder on the face. (9.556)

rip-bol-a $n$. full immersion baptism, for Baptists. (8.564)

rip-e-a, rip-i-a $v t$. carry on the shoulder, as a log. (9.532)

rip-eng $n$. friend, (A•chik). (6.83)

rip-eng-dam-beng $a d v$. this way and that, inconsistent, disorderly, going here and there, in speaking, in moving about. (3.718)

rip-ing-rip-ang $a d v$. disorderly, thrown here and there, of objects, of messily planted rice, of speaking in a disordered way. (3.718)

rip-om-a vi. swell up, of parts of the body. (8.956)

rip-on-chi $n$. dew. (4.329)

rip-pong-rip-pong $a d v$. flying about in large quantity, of such things as feathers, cotton. (9.216)

rip-rip-a, mik-ka rip-rip-a vi. rain a few drops. (4.325)

rip-u-a vt. dry out over a fire, of meat, fish: wak be-en-ko wal-o rip-u-a pig meat dries at the fire. (9.272)

rit-a vt. boil. (5.46)

rit-a $v t$. roast rice with some water to prepare it for pounding. (5.451)

rit-a, stil rit-a vi. rumble, sound of hail preparing to fall; boil. (4.329)

rit-am-a vt. trim, cut off larger branches of a standing tree. (9.573)

rit-cha-sa num. one hundred, (mainly A•chik). (3.3799)

rit-chu-a vt. join, attach together, as bamboo pieces, sections of house beams, cloth by sewing. (9.63)

rit-dang-ban-dang $a d v$. disagreeing, speaking at the same time, confused, tangled, unsystematically, disorderly. (8.32)

rit-ek-a-rit-ek-a vi. sick and weak and unable to get up. (8.93)

rit-im-a, rit-im-pil-a vi. grow, get new leaves or bark, sprout, as from a tree stump; grow new skin, of a snake. (4.691)

rit-ok-rit-ok $a d v$. falling by themselves, of fruit. (4.694)

rit-ti-rit-ti, rit-ti-rit-ta, rit-ti-rit-tak, rit-ti-ti-rit-ta-ta $a d v$. rain just a bit, intermittently, here and there, drop by drop. (4.325)

rit-tik-e-rit-tak-e ga--ak-a $a d v$. rain just a bit. (4.325)

rit-tong-ban-tong $a d v$. speaking quickly and loudly, as when arguing. (7.14)

-ro ts. indicates a correction to a previous statement or presumption: ang-a Chu-ni-a-cha i-ang-jok-ro I did too go to Chunia. (0.31)

ro-a, ru-a $n$. rice seedling after removal from seedbed or after transplanting: ro-a sik-a transplant rice. (5.25)

ro-a, ru-a vi. mate, of animals, not used for people. (4.482)

ro-a, ru-a vt. dance, as a kir-ton. (8.83)

ro-a-tik-bi-du, ru-a-tik-bi-du $n$. a thin vine with much indented fernlike or fingered leaves, the middle finger being longest. (4.655) 
ro-am-a, ro-om-a, ro-o-a vi. wander, stroll. (9.319)

ro-ar pam, ro-al pam $n$. rower pump, water pump that is pulled with an oar-like handle. $<$ E $(5.838)$

Ro-bi-bal, Ro-bi-bar $n$. Sunday. $<\mathrm{B}(2.811)$

ro-chok-a, ru-chok-a vi. long and pointed, of a face. (6.42)

ro-gat-a, ru-gat-a vi. dance done at a sacrifice for the spirits when the dancers return to the house from the altar. (8.83)

ro-kom, ro-hom $n$. kind, type, variety. $<\mathrm{B}(3.437)$

ro-kom- cls. classifier for kinds, varieties. $<\mathrm{B}(1.31)$

ro-rim-a vi. come or go in large numbers, in a crowd of people, cattle, etc. (9.319)

ro-rot-a, ru-rot-a vi. cackle, sound made by a hen when she lays an egg. (4.476)

ro-se-a, ru-si-a vt. choose, sort, select out the bad ones. (9.536)

ro-si $n$. the rope that attaches the yoke to the plow. $<\mathrm{B}(5.87)$

ro-sun, ro-sin, ra-sin, na-sin $n$. onion, garlic. $<\mathrm{B}(5.23)$

ro-sun gip-bok, na-sin gip-bok $n$. garlic. (5.23)

ro-sun git-chak, na-sin git-chak $n$. onion. (5.23)

ro-ti, ru-ti $n$. bread. $<$ B (5.408)

ro-a, ru-a vi. long in space or time, of roads, houses, stories, time periods, etc.; tall, of people, trees, posts. -ro--, -ru-aa. long: mangro-a long in the body; chang-ro-a tall, of people, trees; ja-ro-a long, of pant legs, shirttails, for a long time. (2.213)

ro'-chok-a, ru•-chok-a vi. long and pointed. (2.312)

ro'-e-ro'-e a-gan-a, ru'-e-ru'-e a-gan-a vi. speak for a long time, say

a lot, stretch it out, speak slowly. (7.14)

ro'-mi-si-a, ru'-mi-si-a vi. very long, in space or time. (2.213)

ro-on-a vt. take down, take out, remove. (9.511)

ro-op-a vi. go down, flatten, of a swelling. (8.956)

ro-ot-ro-ot dak-a, dam-bu ro'-ot-ro-ot adv. big, of a baby. (6.44)

ro-rek-a vi. long and thin, of cylindrical things, shaped like bamboo. ro-rek-rek, ru-rek-rek $a d v$. long of cylindrical things, smaller in diameter than ro-rong-rong. (2.312)

ro'-reng-a, ru'-reng-a vi. long, of cylindrical things, sticks, pens. (2.312) ro-reng-ro-reng $a d v$. long and beautiful. (2.213)

ro'-ring-ro'-ring, $\mathbf{r u}^{-}$-ring-ru'-ring $a d v$. stringy, like raw egg or runny dough; running, of sap, dripping egg white; stretched out in time, of a tune. ro-ring-a, ru'-ring-a vi. (9.257)

ro'-rok-a, ru•-rok-a vi. somewhat long, of body parts, fingers, face, arms, legs, nose; somewhat elongated, like a banana, pineapple; tall, of trees; not as long as ro-reng-a, neither very long nor very short. (6.44)

ro'-rong-a, ru'-rong-a vi. cylindrically shaped, like a tin can, the trunk of a papaya tree. ro-rong-rong $a d v .(2.312)$ 
rok, ja-si-ni rok, jak-si-ni rok $n$. veins, tendons of the toes, fingers. $(6.233)$

rok-a $v t$. scrape, scrape off, as a dirty pot; shave or cut hair, with scissors or razor; push rice into the hole of a teng-ki (rice pounder) with an al-pat (push tool).ma-rang rok-a vph. remove ma-rang (evil and pollution); dispose of ma-rang by means of an egg sacrifice using bamboo that has been shaved so as to make a ku-sim-ang (beard). -rok- $a a$. scrape, rub, scrape off, knock off, remove: dok-rok-a knock one thing against another, as to clear dirt from a basket; rep-rok-a cut off peel; dang-roka scrape out, as dirt or weeds; rep-rok-a carve off bits from the surface; ot-rok-a sweep. (9.557)

rok-ra-bi-du $n$. slender vine with long narrow leaves. (4.655)

rok-rak-a vi. loose, not tight, as a tool handle; wobble, of a pole in the ground; loosely tied, loose, of ripe fruit on a tree. rok-rak-ga-rak adv. $(2.23)$

rok-rek-a vt. loosen, shake loose, as a tooth, a pole in the ground, tool handle, furniture; loose and rattling, of the seeds of su-ri fruit; make something move back and forth, like a post in a hole. rok-rek-ga-rek $a d v$. loose, not tight, as a handle. (2.23)

rok-rek-ga-rek $a d v$. loose, not tight, as a handle. (2.23)

rok-rek-it-a vt. cause to move back and forth, of a tree or post that wobbles. (2.23)

rok-rok, rok-rok cha'-a $a d v$. eat up, finish: ang-a rok-rok cha'-tokjok I ate up everything. (5.479)

ron-a, on'-a, hon-a vt. give, put; apply, as paint; pay a fine, compensation; give a loan; po-ri-ka ron-a take an examination (what students do), hand or give in the exam; bi-a ron-a perform a marriage, officiate at a marriage. (9.72)

ron-bek-a $v t$. give forcefully, even against the will of the receiver. (9.72) ron-chak-a, on'-chak-a $v t$. give help, with money, rice, etc.; contribute, as food for a wedding. (9.73)

ron-kam-a, on·-kam-a vt. give something that is not to be returned, give finally. (9.72)

rong $n$. color, variety, type; suit in cards. $<\mathrm{B}(3.539)$

rong rak-a $v p h$. be permanent color, strong color. (3.539)

rong- cls. classifier for round and spherical things, for large pots, fruit, eyes, coins. (1.32)

rong-bet, rong-bret $n$. cracked rice, a snack food made by frying cracked rice dry, without water or oil. (5.403)

rong-bet gin-di, rong-bret gin-di $n$. fried cracked rice, cracked finer than ordinary rong-bret, but not as fine as flour. (5.403)

rong-bok-a vi. rice that is almost but not fully ripe, riper than bri-a. $(4.692)$ 
rong-chon-a vi. small, of round things, fruit, eyes, pots. (2.211)

rong-chon-mit-ing $a d v$. while small, an early ga-dom stage of maturation. (4.692)

rong-chu $n$. flattened rice that is eaten as a snack. (5.403)

Rong-chu-gal-a $v t$. a traditional festival that involves the throwing of rong-chu (flattened rice). (8.562)

rong-dal-a vi. big, of round things, fruit, eyes, pots, breasts; to swell up, grow round, of fruit, breasts, sores; rong-dal-ing-a growing round and large, of fruit, of breasts, as a stage of maturation, jokingly of testicles: tor-mus ko-mil-a-na-bat-e rong-dal-a watermelons are bigger than oranges. (2.211)

rong-dik $n$. earthenware storage pot with no neck and a large mouth, used to store rice; the thick part at the base of a banana trunk, (lit. banana-round pot). (5.432)

rong-ge-rong-ge $a d v$. a long time, late. (2.53)

rong-get-a vi. bulge, have a roundish protrusion; pregnant. (2.211)

rong-git-chak-a vi. almost ripe, especially of rice, riper than bri-a. (4.692)

rong-git-ing $a d v$. not fully cooked, not yet ready to eat, of rice. (5.46)

rong-git-ting rong-min-ek adv. misunderstand, (lit. undercookedovercooked). (7.41)

rong-gop-rong-gop adv. relatively large, of small round things such as ripe fruit, rice grains, sugar, thread. (2.211)

rong-got $n$. joking about a little boy's testicles. (7.26)

rong-gri-rong-gra $a d v$. not sticky, (same as gra-a). (3.668)

rong-kal-a vi. joke, tease, fool around. (7.26)

rong-ma-rong-ma $a d v$. large of round things when there are more than one. $(2.211)$

rong-mi-sep $a d v$. small, of round things, of hail stones. (2.211)

rong-min-ek $a d v$. well cooked, done, of rice. (5.46)

rong-ot-a $v t$. take out something round, as a banana from its skin, an eye from its socket; dig up; expose the genitals. (9.572)

rong-rang-a vi. loosely fitting, as bracelets. rong-rang-ga-rang adv.: tang•-rong-rang-a loosely fitting. (2.23)

rong-reng, tam-pi-rong-reng $n$. big blue fly that eats dung, horse fly. (4.462)

rong-reng-a, na-rong-reng-a vi. shake head, indicate 'no'. (9.41)

rong-ret-a vi. out of shape, sideways, twisted, often of a body part, leg, arm, finger; dislocated, of bones; not meeting, of roads, of people going in opposite directions; wrong way in traveling. rong-ret-ga-ret $a d v$. inconsistent, disorganized, as when speaking in an inconsistent and disorganized way. (2.319) 
rong-rong, rong-rong dak-a $a d v$. late, delayed, take a long time. (2.53) -rong-rong- dns. clean, pure: gip-bok-rong-rong pure white; chirong-rong clean, clear; git-chak-rong-rong bright red. (3.714)

rong-rong-a vi. leak, as from a sack; fit in or through a hole. -rongrong- $a a$. through a hole: gip-u-rong-rong-a fit through something small, such as a sieve; ga•-ak-rong-rong fall through; ki·-rong-rong-a have diarrhea. (2.22)

rong-sep-a vi. having small eyes, like Mandis. (6.42)

rong-tal-a vi. clean, clear, $(\mathrm{A} \cdot$ chik $)$ : gi-sik rong-tal-a clear in the mind, know. (3.714)

rong-tal-gip-a $n$. holy spirit, (lit. clean one). (8.54)

rong-tek $a d v$. not fully cooked, still somewhat hard, of rice. (5.46)

rong-ting-gi-rong-tang-ga, rong-ting-rong-tang adv. neither very hard nor very soft, as rice cooked without enough water. (3.664)

rong•- $c p$. prefix for stones: rong•-kol cave; rong•-krek small lumps of hard earth; rong-te rock. (0.17)

rong•-gok-a vi. break off, of a round thing, such as a glass bottom, the neck of a pot. (9.282)

rong-kat-a vt. take out, remove from. (9.572)

rong•-kol $n$. cave. (4.231)

rong•-krek, rong·-kek $n$. small lumps of hard earth. (4.244)

rong-te, rong•-ti $n$. stone, rock, big stone; the flat (lower) grindstone; the weight used on a balance scale. (4.244)

rong•-te-ni $\mathbf{p i}^{\cdot-\mathbf{s a}}$, rong•-te $\mathbf{p i}^{-}-\mathbf{s a} n$. the smaller (upper) grindstone that is held in the hands, (lit. stone's child). (5.438)

rot-a $n$. small round metal or earthenware water pot. $<\mathrm{B}(5.432)$

rot-a-rang-tal $n$. dishes, cooking utensils. (5.434)

ru-a $n$. axe. (5.832)

ru-a vt. pour liquid, slower and more carefully than pak-a; take down, take apart, of thatch, a fence, a basket; take down a house. (9.276)

ru-a, ro-a $n$. rice seedling after removal from seedbed or after transplanting, rice plant: ru-a sik-a transplant rice seedlings. (5.25)

ru-a, ro-a vi. mate, of animals, not used for people. (4.482)

ru-a, ro-a vt. dance, as a kir-ton. (8.83)

ru-an $n$. winnowing tray with curved up sides and back, but a flat and open end. (5.852)

ru-ang-ru-ang $a d v$. see or hear dimly, in the distance. (3.54)

ru-ang-ru-ang, gi-sik ru-ang-ru-ang $a d v$. very lonely, miss strongly, homesick, deep sorrow, deep thinking, anxiety; the way one feels with some kinds of music. (7.743)

ru-at $n$. land leech, smaller than a water leech. (4.466)

ru-brap-a $v t$. pour over. (9.276) 
ru-dap-a vt. pour over, as water over the head when bathing. (9.276)

ru-dit-a $v t$. pour a bit of rice beer onto the ground as an offering for the mit-e (spirits). (8.562)

ru-gal-a, ru-e gal-a vi. ceremonial offering of rice beer to spirits, by pouring and throwing. (8.562)

ru-gek-a vt. filter ashes to get soda, pour a large quantity. (5.458)

ru-gi, ro-gi $n$. sick person, patient. $<\mathrm{B}(8.91)$

ru-kim-it-a $v t$. pour on (water) to put out fire. (5.424)

ru-kring-krang-a $v t$. take down old thatch, pouring it down all around. (5.632)

ru-mal $n$. handkerchief, small cloth. (6.521)

ru-pa $n$. silver. $<\mathrm{B}(5.88)$

ru-prak-a vt. pour lots of water, as from a bucket over the head when bathing: chi ru-prak-e a-bu-ing-a bathe by pouring water. (9.276)

ru-ra-a $v t$. move an object from one place to another; irregular, inconsistent. (9.511)

ru-ra-ga-ra $a d v$. inconsistent, in speaking or moving; move back and forth; irregular. (9.315)

ru-rat-ru-rat, ru-rat-ga-rat $a d v$. back and forth. -ru-rat-, -ru-ra'- $a a$. back and forth: bil-ru-rat-a fly back and forth; i $\cdot$-ru-rat-a go back and forth, commute; don-ru-ra'-a move things back and forth; mal-ru-ra'-a crawl back and forth. (9.315)

ru-reng-reng $a d v$. lie out straight and quiet, knowing nothing, in sleep, sickness, or death. (7.96)

ru-rong-a $v i$. fall, of leaves, over-ripe fruit, badly tied thatch; tumble down, as a pile of firewood. (9.212)

ru-rot-a vi. fall through, leak while being moved, as through a hole in a bag being carried. (2.22)

ru-rot-ru-rot $a d v$. falling here and there, as bits from a torn sack, from a running nose, of a child's drool, blood from a wound. (2.14)

-ru-ru- $a a$. for a long time, forcefully: kim-ru-ru-a thunder that seems to move across the sky; dong-ru-ru-a be in the same place for a long time; ak-ru-ru-a pluck for a long time. (2.53)

ru-srip-a $v t$. pour off water leaving solid sediment behind, decant. (9.276) ru-tring-trang-a vt. take completely apart, of a roof, fence, house, mat. (9.65)

ru'-a, ro'-a vi. long, tall, (see ro'- for compounds). (2.213)

-sa dns. a pair of kinsmen: at-chu-sa grandfather and grandchild; mama-sa mother's brother and sister's child; ma-ni-sa mother-in-law and child-in-law. (6.62)

-sa num. one. (3.3701)

sa qw. who?: sa-ni nok whose house?; sa-na nam-nik-a? who (does she) like?; sa-sa neng-ing-a who all is tired? (A•chik: sa-wa). (1.21) 
-sa, -ha ns. only. (0.14)

sa-a vi. in pain, sick. (8.91)

sa-a vt. serve, pass out food; spread out food to distribute; spread out rice on a mat when making rice beer. (5.458)

sa-ban $n$. soap. $<\mathrm{B}(6.57)$

sa-chak-a $v t$. echo in ron'-chak-a sa-chak-a contribute. (9.73)

sa-di-a int. dear me! (0.64)

sa-du, sa-du-gip-a $n$. wife's sister's husband: sa-du dal-gip-a wife's older sister's husband; sa-du chon-gip-a wife's younger sister's husband; sa-du-sa husbands of sisters. (6.614)

-sa-ge, -sa-gi dns. former, deceased: Rong-jeng-sa-ge the deceased Rongjeng. (6.828)

sa-gip-a $q w$. who?; which?, of several people; sa-gip-a-ko nik-jok which person (did you) see? (1.21)

sa-ma-po-ki $n$. a robin-like bird, said to be able to talk, (Beng. sa-ma). $<\mathrm{B}(4.43)$

sa-mi-sa-a, cha-mi-sa-a $v t$. arouse from sleep, wake someone up. (7.97) sa-nai $n$. small reed horn, made of wood with a flared end. (8.85)

sa-pak-a vt. distribute cooked rice from a pot before it is wrapped, put out on plates. (5.458)

sa-ra, cha-ra $p p$. except for, other than, instead of, without, (follows nominative): wak sa-ra: without pigs. $<$ B (1.16)

sa-rang, mi-sa-rang $n$. a traditional kind of hill rice, one kind of miman-di. (5.25)

sa-ren-di-a vi. somewhat sick. (8.91)

sa-ri $n$. sari, woman's wrapped outer garment. <B (6.526)

sa-ri $n$. sister-in-law, used reciprocally between any sisters-in-law. (6.615)

sa-ri-a-ni $n$. mothers-in-law, daughters-in-law and sisters-in-law. (6.62)

sa-rong $n$. smallish water pot. (5.432)

sa-sa $q w$. who all? (1.21)

sa-sat $n$. an incense made from the wood of the sal tree and burned for its fragrant smoke in some Sang-sa-rek ceremonies: Sa-sat so-a the ceremony in which sa-sat is burned. (8.58)

sa-wa $q w$. who? (A•chik). (1.21)

sa-wi-a vt. insult, scold, bawl out, give bad words. (7.27)

sa-wil $n$. a large vine with yellow flowers and a big fruit; used for pot scrapers. (4.655)

sa-a vt. build a nest, build a hive, by birds or bees, on a branch or on the ground but not a hanging nest. (4.482)

sa--al-a $n$. swell up, as rice when cooking. (5.46)

sa-dik-a vi. hurt, ache, be painful; burning taste of chilies. (8.94)

sa-dik-srim-a vi. a little painful, not very painful. (3.64) 
sa-kam-a vi. painful. (8.94)

sa-tip $n$. afterbirth. (6.29)

sai-kel, bai-si-kol $n$. bicycle. $<\mathrm{E}(5.872)$

sai-tan $n$. kind of fish, (Beng. ta-ki). (4.454)

sak $n$. greens, edible vegetable leaves. $<\mathrm{B}(5.409)$

sak-, ak- cls. classifier for people. (1.31)

sak-a $n$. upper place, upland. (2.11)

sak-a vt. braid hair or string; make rope; spin; twist cloth, etc. (6.25)

sak-a-gil-gil $a d v$. shallowly, of plowing. (2.215)

sak-a-ni ku'-chil $n$. upper lip. (6.22)

sak-a-ni wa $n$. upper teeth. (6.22)

sak-a-ni wen-a $v t$. weave the top part of a basket. (5.856)

sak-a-o, sak-a-cha $p p$. above, on top of, beyond, (follows genitive -ni):

nok-ni sak-a-o on top of the house. (1.11)

sak-a-pong, sak-a-pong-o $p p$. top, on top, on the surface of, (follows genitive -ni): chi-ni sak-a-pong-o on the surface of the water. (1.11)

sak-ki $n$. witness at a legal case. $<\mathrm{B}(8.13)$

sak-ki-a vt. strain, as milk, to remove dirt, etc. (5.458)

sak-sa-han, sak-san num. only; alone, of people. (3.344)

sak-sel-a, sak-sel-sel-a $v t$. twist string, cord, bamboo tie strips. (9.645)

sal $n$. sun, day, daytime; 24 hour period. sal- cls. classifier for days.

$(4.214)$

sal ding -a $v p h$. sunlight, as opposed to shade. (3.554)

sal min-ok-a, sal-ko min-ok-a vph. eclipse of the sun, (lit. swallow the sun). (4.214)

sal na-na skang $n p h$. before sunrise. $(2.52)$

sal si-a $n$. end of the day, (lit. sun dies). (2.52)

sal wel-a vi. the time past noon. (2.52)

sal-a int. brother-in-law, a scolding word, used as an insult. $<\mathrm{B}(0.66)$

sal-a vt. pull; pull in order to lengthen, stretch; play a harmonium; draw a line; pump a 'rower' pump; adjust a sari; conduct a legal case, pull out the arguments. -sal- aa. stretch, pull: han-sal-a stretch the body, as after sleeping; sur-sal-a a slow, stretched-out singing style. (9.511)

sal-a-gip-in $n$. another day; sal-a-gip-in i-ba-bo come on another day. $(2.65)$

sal-a-jring $a d v$. every day. (2.71)

sal-a-ram $n$. east, (A•chik). (4.219)

sal-a-rik-kit $a d v$. every day. (2.71)

sal-an-tam-o $n$. time when the sun begins to move lower in the sky, late afternoon. (2.52)

sal-an-ti adv. every day. (2.71)

sal-bret-bret $a d v$. not very hot, comfortable. (3.673)

sal-chak-a $v i$. warm oneself in the sun. (3.673) 
sal-chet-chet-a vt. pull tightly. (9.511)

sal-chi-bil-a vi. not hot, of weather on a cloudy day. (3.673)

sal-chrit-a vi. striped, as striped cloth, as a tiger. (3.538)

sal-chu-gra-gra $n$. time when the sun is well up, but not way up, one or two hours after sunrise. (2.52)

sal-ding-dang-a vt. pull in various directions. (9.511)

sal-do-a, sal-du-a vt. pull up, as to draw water with a bucket from an open well. (9.511)

sal-do-det-a vt. pull back and forth. (9.515)

sal-gal-a vt. pull away, pull off; put away, as a blanket. (9.68)

sal-gi $n$. heaven. $<\mathrm{B}(8.52)$

sal-gim-ik $a d v$. the whole day. (2.53)

sal-gip-eng, sal-gip-eng-pang $n$. south, $(\mathrm{A} \cdot \mathrm{chik})$, the southern (shorter) path of the winter sun. (4.219)

sal-gom $n$. a large kind of radish, a round, red and bulbous root crop. $(5.27)$

sal-gom-a $v t$. pull down, bend down, as tree branches. (9.511)

sal-gon-da-ri $n$. a long and striped kind of fish, (Beng. go-sul). (4.454)

sal-gro $n$. north, $(\mathrm{A} \cdot \mathrm{chik})$; the northern (longer) summer path of the sun. (4.219)

sal-ha chang-e-chang-e $a d v$. every alternate day. (2.71)

sal-i-bot-a vt. drag something along the ground. (9.511)

sal-i-ram $n$. west, $(\mathrm{A} \cdot$ chik $)$. (4.219)

sal-iis $n$. gathering of people to settle a legal case, (same as bi-char). $<\mathrm{B}(8.13)$

sal-ik-kim $n$. shade, as offered by a tree, house, etc. sal-ik-kim-a vi. be shady, become shady. (3.558)

sal-jat-chi, sal-jang-chi-o, sal-jat-chi-o $n$. in middle of the day, noon. $(2.52)$

sal-jot-jot-a $v t$. pull hard, as when tying a string. (9.511)

sal-jret-jret-a vt. pull tightly. (9.511)

sal-kil-ak-a vt. pull open, pull up sleeves, open shirt front. (6.53)

sal-kring-a $v t$. stretch out, of arms, cloth, string. (9.642)

sal-nam int. somewhat artificial translation of English 'good day'. (0.64)

sal-ok-a vt. pull out. (9.511)

sal-on-a vt. pull down. (9.511)

sal-pak-sa $a d v$. half a day. (2.53)

sal-prak $a d v$. all day, the whole day. (2.53)

sal-sa dong-e $a d v$. omitting one day, after skipping one day. (2.53)

sal-sa-wal-sa $n$. one day and one night. (2.53)

sal-sek-a, sal-ek-a vt. pull someone away; take back a woman by a wife's

kin when they dislike her husband. (6.655)

sal-si-si $a d v$. all day, the whole day. (2.53) 
sal-srik-a vt. make smooth, as by scraping a knife on bamboo, clean; scrape rice into the hole of a rice pounder. (9.554)

sal-srip-a, sal-sip-a vt. slurp, suck; sniff to smell, sniff with the nose for something that smells good; sniffle with a cold; suck water with trunk, of an elephant. (5.472)

sal-tat $a d v$. all day, the whole day. (2.53)

sal-tet-a vt. break by pulling, as a string. (9.284)

sal-tik-a $v t$. pull on, pull out, esp. of hair. (9.511)

sal-a $n$. a soft and flexible broom, used for indoor sweeping. (5.835)

-sam dns. side, beside: ha-sam side of a field; nok-sam area beside a house; chi-sam water edge. (0.16)

sam $n$. grass, herb; medicine; curry. sam- $c p$. prefix for grass and small herbs: samsi crab-grass; sam-mik-chip sensitive plant. (4.652)

sam ba-si-a $v p h$. remove weeds; sort. (5.156)

sam bit-e, sam bit-i $n$. a little round burr. (4.676)

sam- cls. classifier for any bilateral body part, hands, eyes, etc. (1.38)

sam-ak-ki $n$. a ragged 1-2 meter plant with $15 \mathrm{~cm}$. fuzzy, dark green, slightly heart-shaped leaves with lighter colored leaves in themiddle. (4.656)

sam-ba-o $p p$. near, beside, from nearby, (follows genitive -ni), (A•chik). (1.11)

sam-ba-si-a $n$. an altar for a sacrifice of any sort: sam-ba-si-a-ko banai-a construct an altar. vt. make an altar for a sacrifice. (8.562)

sam-bi-jak $n$. vegetables, (lit. grass-leaves). (5.409)

sam-bi-si $n$. poison mixed with food to kill someone, as by a gu-ni-al. $(8.58)$

sam-bol $n$. medicine that is drunk or injected. (8.99)

sam-cheng, sam-cheng bol-cheng $n$. old unwanted plants and leaves that have fallen from trees, rubbish. (4.66)

sam-dap $n$. wrapped up portion of curry, serving. sam-dap-a vi. wrap up curry portions in banana leaves. (5.453)

sam-dik $n$. curry cooking pot. (5.432)

sam-gol-dak $n$. small wild woody shrub, a foot or so high with edible but somewhat bitter leaves. (4.64)

sam-ja-lik $n$. a small pinkish-flowered plant with clover-like leaves, small five-petaled yellow flowers, and a red stalk, inedible. (4.656)

sam-jang-gi $n$. low herb with $8 \mathrm{~cm}$. slightly indented leaf; the rubbed leaf is good on wounds, (Beng. pa-tor-chu-na). (4.656)

sam-mik-chip $n$. sensitive plant, a little plant with tiny leaves that close up when touched. (4.656)

sam-pal $n$. bundle of curry, (same as sam-dap). (5.453)

sam-pil n. comb for the hair. (6.57) 
sam-si $n$. crabgrass. (4.652)

sam-sim-a-ri $n$. tall plant with little, slightly violet, everlasting-like flowers. The stems are used to make light fences, and crude walls and partitions, and the crushed leaves are rubbed on wounds. (4.656)

sam-su-eng $n$. small, slightly smelly herb. (4.656)

sam-tak-a-ri $n$. little herb with small white flowers in clusters, much indented leaves, and burrs. (4.656)

sam-tam-pret $n$. a jungle plant, used by an herbal doctor (ko-bi-raj). (4.656)

sam-ti, sam-ti-bi-te $n$. a 1 meter high plant with round burrs that are smaller than a pea but that catch clothing, different from sam-tak-a-ri. (4.656)

san-da $n$. film over the eye, cataract. $<$ B (8.92)

san-di-a vt. look for, search: gim-a-ko san-di-a look for something lost. (3.514)

san-tok $n$. old-fashioned broad silver wrist bracelet. (6.564)

sang-gong $n$. woman's bracelet, bangle; plant tendrils. (6.564)

sang-gong, bi-dil-ni sang-gong $n$. tendrils, (lit. bracelet of vines). (4.670)

Sang-sa-rek $n$. the Mandi name for the traditional religion of the Garos, (A·chik: Song-sa-rek). (8.51)

sang·-chak-a vi. brave, courageous. (7.746)

sang-et, sang•-ret $n$. firefly. (4.462)

sap-a vi. know how to, be good at, be expert at; do systematically and with good results. (7.55)

sap-a vi. swoop down, dive, as hawks swoop down to grab meat. (4.486) sap-srak-a $v t$. dive to catch, as a hawk catches baby birds: du・-reng du'-pi-sa-ko sap-srak-a the hawk dives to get the baby bird. (4.486)

sar $n$. fertilizer, either natural manure or commercial. $<\mathrm{B}(5.835)$

sat-a vt. ladle out, scoop out, as water when fishing. (9.277)

sat-pak-a vt. scoop out water with bigger scoops than for chok-wak-a. (9.277)

sat-ti $n$. umbrella. $<\mathrm{B}(6.521)$

sau-sau-e $a d v$. softly, of thunder that anticipates rain. (4.342)

se, si, se-gip-a, si-gip-a $n$. husband. (6.614)

se-a, bi-mik se-a vt. scrape bi-mik decorations on the green surface of bamboo; write, $(\mathrm{A} \cdot$ chik). (5.856)

se-a, si-a $n$. write, $(\mathrm{A} \cdot \mathrm{chik}) .(7.28)$

se-bit-a vt. decorate bamboo by scraping and making a ku·-sim-ang (beard). (5.856)

se-eng-a vi. smell bad, stink, (A·pal, A•chik), (same as so-eng-a). (3.688) se-gal-a, se-ko gal-a vt. divorce, (lit. throw away one's husband). (6.655) 
se-jik $n$. couple, husband and wife, less used than jik-se. (6.62)

se-ni $n$. a small tool used for chopping out weeds that has a wooden handle and a flat iron blade with a curved cutting edge pointing away from the handle. (5.831)

se-pang, se-pang-o, si-pang, si-pang-o, si-pang-cha $p p$. near, close by, (follows genitive - $\mathbf{n i}$ ). (1.11)

se-pang-grik $a d v$. close to each other. (2.11)

se-pang-se-pang $a d v$. in the surrounding area, nearby. (2.11)

se-ra-be-ra $a d v$. confused; every which way; jumbled and messed up. $<\mathrm{B}$ (3.718)

se-ram-pu $n$. a very small red or black ant, (same as sim-il-chak). (4.465)

se-re-jing $n$. a light-hearted type of song based on the story of Serejing and her lover, Waljam. (8.83)

se-reng-ki bu-su $n$. thorny tree with small sour leaves that are cooked with curry. (4.632)

se-ru, se-ruk $n$. any of several kinds of myna birds. $<$ B (4.43)

se-ru-a-bil-ek $n$. a kind of myna bird, (grayheaded myna). (4.43)

se-ru-a-pal $n$. kind of myna with yellow eyes, and habits like an American robin, a bit smaller than the similar common myna. (4.43)

se-ru-gin-ti $n$. medium-sized myna. (4.43)

se-ru-man-di $n$. common myna bird with yellow eyes and the size and habits of an American robin, lucky bird, (Beng. sal-iik). (4.43)

se'-em-a, sem-sem-a vi. tiny, like millet grains or mustard seeds; fine, of powder; thin, of cloth; fine rain, drizzle. se·-em-chik-chik, se·-emse•-em, sem•-sem dak-a $a d v .(2.215)$

se•-em-se•-em, sem•-sem dak-a $a d v$. fine rain, drizzle. wa-sem-sem-a vi. (4.325)

se-et-a vt. pinch something between two fingers, either to hurt it or to hold it. (9.531)

-sek- $a a$. by force, forcefully: sal-sek-a pull away, take someone by force; jik-sek-a steal a wife, take someone else's woman; ra'-sek-a take by force. (7.56)

sek-a vt. have sex in violation of proper rules, either premarital or adultery. (6.72)

sek-ki $n$. sap, tree sap. (4.674)

sel-a vi. leak, of liquids through a roof, a pot, etc. (9.276)

sel-gol-gol-a vi. leak a lot, rapidly. (9.276)

sel-o ma-sin $n$. diesel pump. $<\mathrm{B}(5.838)$

sel-a vi. improve in health, recover. (8.91)

sel--pu-a vi. stand on end, of hair. (4.482)

sel-sel-a vi. echo in del--del-a sel-sel-a have the sound of a broken machine, bad drum head. (3.624) 
sel•-uk $n$. cockroach. (4.469)

sen-dil $n$. sandal. $<\mathrm{E}(6.523)$

sen-dur $n$. colored cosmetic spot on forehead. $<\mathrm{B}(6.565)$

seng-chok-a vi. upside down, with the head down: seng-chok-e ong-on-a come down headfirst, as a woodpecker or squirrel. (9.212)

seng-so-a, seng-su-a vt. wait for. (9.328)

seng-su $n$. large tree with small leaves. (4.632)

seng•-a vi. bright, brilliant, shine, grow light as at dawn; alert, smart, clever. (3.554)

seng•-na git-tu $n$. early morning, morning twilight. (2.52)

seng-nik-a $v t$. feel smart about oneself; show off. (7.43)

seng·-o ki-a vph. be pregnant. (6.74)

seo-seo-e bol-a $v p h$. having a hoarse voice. (7.15)

sep-a, sep-jret-a vt. wring out water, squeeze out water from wet clothes; squeeze out juice from fruit; milk a cow. (9.277)

sep-pi, spi $n$. warrior's shield. (5.845)

sep-u-a $v t$. press out, squeeze out, as pus. (8.99)

ser-, sel- cls. classifier for seers, an Indian unit of weight, approximately one kilogram. $<\mathrm{B}(1.36)$

ses $a d v$. done, finished, last. < B (9.14)

set-ta-a vi. improve in health. (8.91)

si-a, se-a vi. write, (A•chik). (7.28)

si-a vi. die; go out, of fire, lamp: git-ok si-a be hoarse, (lit. throat dies); gro si-a settle a legal case; ja-jong si-a dark of the moon, (lit. moon dies). (6.79)

si-a vi. echo in tok-a si-a spotted. (3.538)

si-a, ju-mang-cha si-a vt. give instructions in a dream in which the dreamer is told what to do: si-pil-a dream again, repeat, of a dream in which instructions are given. (7.96)

si-chang-a vi. die in the middle of life, die ahead of time. (6.79)

si-dil-a $v t$. neglect, fail to care for, (lit. lead in dying). (9.73)

si-dip-it-a vi. partly dead, but still a bit green, of plants. (4.696)

si-et-a, si-it-a vt. kill, cause to die, put out, of hurricane lamp, etc.: gro si-et-a settle a case, (lit. cause the case to die). (6.79)

si-get, si-gret, si-ga-ret $n$. cigarette. $<\mathrm{E}(5.429)$

si-git-ik-a vi. die suddenly. (6.79)

si-kal dak-a, si-kar vt. hunt, go hunting. <B (4.49)

si-kal-tim-a $n$. hunt quietly, stealthily. (4.49)

si-kan-di-a vi. die before time, before full age. (6.79)

si-ne-ma $n$. cinema, movies. $<\mathrm{E}(8.83)$

si-ri, sri, sre $n$. tongue, (see sre for compounds). (6.22)

si-ri ang.-ki-sa dak-a $v p h$. have a sore on the tongue. (8.94) 
-si-ri- aa. gentle, into small pieces: pit-si-ri-a split into small pieces; bi-si-ri-a cracked finely, of glass, cup, pot, etc; ja•-si-ri-a on tiptoes; gil-si-ri-a shed skin, peel off bark. (3.347)

si-sa $n$. lead (the metal). (5.88)

si-sa-a vi. burn in the mouth from eating chilies, burn on the skin from touching chilies. (8.94)

-si-si $d n s$. all, whole, very; song-si-si whole village; ap-si-si very narrow; sal-si-si all day. (3.343)

si-sim-ak-a vi. faint, (lit. die-wake up). (7.96)

si-ti-ri-bi-dil, si-ti-ri-bi-du $n$. a thin wild vine with $3-5 \mathrm{~cm}$. edible scalloped leaves with indented bottom, and many-branched small greenish whitish flowers. (4.655)

si-wil, si-wil-si-wil adv. quietly, softly, so as not to be heard. (3.627)

$\mathbf{s i}^{-}-, \mathbf{s i}, \mathbf{s i}^{-}-\mathbf{i} n$. female genitals. $\mathbf{s i}^{-}-c p$. prefix for female genitals and their parts: si-sim-ang female pubic hair; si-ku-chil genital labia. (6.249)

si-chu $n$. cactus. (4.656)

si-chu, chi'-chu $n$. bladder. (6.27)

si-dal-dal-a vi. an insult for women, large vulva. (7.27)

si-dam-bu-bu $n$. insult for a woman, (lit. big vulva). (7.27)

si-jang-bong-got $n$. an insult for a big-bellied little girl. (7.27)

si--jel $n$. a large variety of jackal. (4.419)

si-ka-ka-rok $n$. a joke for little girls. (7.26)

si-kot-i n. clitoris. (6.249)

si-ku-chil $n$. vaginal labia. (6.249)

si--mik-del-bu $n$. insult to a woman. (7.27)

si-na-wang $n$. insult for a women, (lit. big mouthed vulva). (7.27)

si-ol, si·-al $n$. jackal, (A•pal). (4.419)

si-pak $n$. inside of a woman's upper thigh. (6.249)

si-sim-ang $n$. female pubic hair. (6.25)

si'-so-brok $n$. insult given to women, (lit. vulva-rotten). (7.27)

si-son-ta $n$. wart, mole, skin blemish. (8.955)

si-sot-ti, si-sot-ta $n$. disease characterized by pea sized pimple-like lumps on the skin. (8.955)

si-tap-pu $n$. somewhat chili-like plant with little yellow flowers, $3 \mathrm{~cm}$. lantern shaped fruit; grows wild and is also a garden weed, but edible. (4.656)

siik-kiit-o $n$. educated. $<\mathrm{B}(8.6)$

siit $n$. bicycle seat. $<\mathrm{E}(5.872)$

sik-a vt. blow, play horn, whistle, flute; use a bamboo tube to blow up a fire. (8.83)

sik-a vt. grab, hold, capture, seize, get, obtain; make a legal case; catch thieves. (9.531) 
sik-a vt. insert, put in, stick in, poke, jab; sew; plant, esp. for rice in mud; put on, wear, of things requiring insertion such as a hat or bracelet; give life, provide the jang-gi (life, soul) by the creator; mik-gil sik-a close eyes, (lit. put into the eyelids). (9.571)

sik-a vt. wish, (A•chik). (7.725)

sik-a, mik-gil sik-a vi. close the eyelids, (lit. insert the eyelids). (9.42)

sik-a-tang $a d v$. willingly, according to one's own wish. (7.725)

sik-brok-a vt. put something into water, cause to be wet, step into water. (9.277)

sik-chap-a vt. sew together. (9.63)

sik-dik-a vt. catch, catch in the act, as a thief; catch someone in adultery. $(9.531)$

sik-do-a vt. push up, push forward, as a flashlight switch. (9.511)

sik-do-do-a vt. push. (9.511)

sik-e ra-a vt. stick in and carry, as in a pocket or bag. (9.571)

sik-gang-pang-a vt. knock over, make a person or animal fall down on its back. (9.513)

sik-jet-a, sik-jet-jet-a, sik-jret-a vt. push hard, press hard; press down, as on a flashlight switch; squash, as an insect; hold down with hands; press in order to squeeze out, as a pimple; tie tightly. (9.67)

sik-jot-a, sik-jrot-a vt. plant between other plants, fill in the gaps in a planted field. (9.571)

sik-keng-krot-a vt. catch and hold tightly, by force, with arms or hands. (9.531)

sik-ket-a vt. insert forcefully, of something tight, insert and leave in, as a hearing aid, a bit into a brace, chunks of mud into the cracks of a drying wall of a new house. (9.571)

sik-kim-it-a, kim-it-a vt. put out, extinguish, of matches, fire, hurricane lamp, flashlight, etc. (5.424)

sik-krok-a vt. put on, wear, of hats, bracelets, rings, socks, shoes, belt; put through, as an arm through a sleeve, the loop a of handbag, an open window; insert, as an object into thatch, or a bit into a brace; hang a loop over something; attach plow in the middle of a jual (yoke); climb into skin, (in mythology). (6.53)

sik-mik-a vt. add a little, as condiments for flavoring. (5.46)

sik-nang-a $v t$. catch in the act, as a thief or adulterer. (8.34)

sik-nap-a $v t$. put into, squeeze in forcefully, as into a house or bus: motor es-es-ten man-di-ko sik-nap-e chak-it-a the bus assistant makes the people fit (into the bus) by squeezing them. (2.22)

sik-on-a vt. push down, press down. (9.511)

sik-pak-a vt. push, shove; knock over. (9.511)

sik-pu-pu-a vt. blow up fire, as by blowing through a tube. (9.45) 
sik-rit-chu-a $n$. sew together, of two pieces of cloth. (6.598)

sik-ru-ru-a, sik-ru-ru-et-a vt. push forward, push hard. (9.511)

sik-sak-gil-ak $a d v$. restless, wiggly, not quiet, constantly moving, as a small child. (9.324)

sik-sep-a vt. stick something into a fold in one's clothing, ear, etc.; tuck or poke in, as shirt at the waist, a tool into rafters, a card into a book; stick in and attach, like a pen with a clip. sik-sep-ga-sep $a d v$. crowded, squeezed in, as in a bus: sik-sep-ga-sep motor ga-hat-a get onto the bus by crowding. (9.571)

sik-sik-a vt. scratch, scrape with finger nails or a tool, to soothe itching, to smooth mud walls; carve, as a memorial post; remove bark, remove the surface of wood, bamboo, etc.; rub against something in order to scratch; go repeatedly, as to a woman in whom one is interested. (9.554)

sik-sim-a vt. put something into liquid, as clothes to soak, sugar to make water sweet, dough into oil. (9.277)

sik-tap-a $n$. sew on a patch, as on a blanket. (6.598)

sik-tat-a $v t$. hit, fight, grab by force in the way sons-in-law were once captured. (6.651)

sil $n$. iron. sil- $c p$. prefix for iron objects. (5.88)

sil-a, jak-si sil-a $v t$. point. (9.46)

sil-ai, sel-ai $n$. gun. (5.845)

sil-chi $n$. a hard kind of iron that takes a good cutting edge and that is used for at-te (bamboo knives), and some other tools. (5.88)

sil-dang-kep $n$. metal tongs, tweezers. (5.436)

sil-eng-si $n$. needle, (same as reng-si). (6.598)

sil-eng-si $n$. kind of ground-dwelling worm that eats cloth, (same as reng-si). (4.467)

sil-eng-si a-kil-ok $n$. eye of a needle. (6.598)

sil-ga $n$. sky. (4.212)

sil-git-chak $n$. rust. (5.88)

sil-git-ting $n$. a kind of iron that is softer and less suitable for cutting tools than sil-chi. (5.88)

sil-it-ing $n$. an ornament of silver, gold or other metal that was worn around the neck by either men or women. (6.563)

sil-kat-a $n$. nail for carpentry. (5.838)

sil-ki $n$. pebbles, small hard glassy black stones. (4.244)

sil-mi-si $n$. small nail, tack. (5.838)

sil-pan $n$. metal collar on the handle of a tool where the knife blade is inserted. (5.87)

sil-pi $n$. singer. $<\mathrm{B}(8.83)$

sil-so, sil-su, sul-so $n$. mustard, mustard seed. (5.23)

sil-so-ni tel $n$. mustard oil. (5.405) 
sil-sok $n$. a little metal cooking tool, with one sharp end for poking and one flat end for stirring. (5.436)

sil-a vt. draw skin back and reveal, draw back the lips to reveal the teeth, retract the foreskin: wa-ki sil-a reveal tooth dirt. (9.44)

sil-ot-a jak-ko sil-ot-a vt. push the hand out of the sleeve. (9.46)

sil-tek $n$. an insult for men. (7.27)

$\operatorname{sim} n$. salt, (variant of kai-sim). (5.405)

sim-a vi. spoiled, smelly, not good to eat, of old rice. (4.696)

sim-a, gi-sim-a vi. dark, black, cold colors, blue. (3.534)

sim-a, sim-a ni-a $v p h$. a procedure in which the names of the mit-e (spirits) are recited while holding a broken black thread which swings when the correct mit-e has been named, thereby revealing which mit-e caused a sickness. (8.562)

sim-ak-a, chu-a-sim-ak-a vi. conscious, awake, wake up. (7.97)

sim-ak-pil-a vi. come alive again. (6.77)

sim-al $n$. tiny red or black ant, (A·pal), (same as sim-il-chak). (4.465)

sim-bra-a vi. miss, be lonely for, worry about, homesick, have nostalgia for. (7.743)

sim-chi-rik-rik $a d v$. pure black. (3.534)

sim-chi-ru-ru $a d v$. pure black; smooth, shiny but not bright. (3.534)

sim-dim-dim $a d v$. dark appearance of trees and shrubs as seen from far away and therefore looking dark. (3.534)

sim-il-a vi. smell pleasant, fragrant. (3.688)

sim-il-chak $n$. tiny red or black ant that swarms in large numbers and often lives in houses. (4.465)

sim-il-e nik-a $v p h$. like the smell of, notice the good smell of. (3.688)

sim-il-ot-a, sim-il-ot-e ni-a $v t$. smell, test by smelling, sniff something. (3.688)

sim-kel-tong-a vi. very black, of people, dogs, etc. (3.534)

sim-ku-ak-a vi. very black, of people, dogs, etc. (3.534)

sim-pru-a vi. very black, pure black, like the soot on pots; very dark, of people, dogs, etc.; very dark complexion. sim-pru-pru. adv. (3.534)

sim-rik-a vi. dark, blackish, ash gray, dark blue, dark green. sim-riksim-rik $a d v$. (3.534)

sim-sak-a vi. careful, think ahead. (7.48)

sim-sam-sim-sam $n$. twilight, partly but not fully dark. (2.52)

sim-sim-a $v i$. the time of growing dark, after the sunset. an-tam-sim$\operatorname{sim} a d v .(2.52)$

sim-sim-sim-sim $a d v$. starting to turn bad but not completely rotten, as old rice. (4.696)

sim•-a, sim•-sim-a vt. soak, immerse, dunk, submerge, as cloth, pots, a tea bag, fish in water so they don't die; wash hair. (9.277) 
sim•-pram-a vi. very sharp. sim•-pram-sim•-pram adv. (3.666)

sin-a vt. like, want to, be attracted to. (7.722)

sin-a, ra-sin-a vt. cut across very smoothly and evenly, of a stick or, esp. a length of bamboo: tam-bing-bing dak-e ra-sin-et cut it off in a smooth circle! (5.856)

sin-a-ru $n$. a poisonous kind of centipede. (4.467)

sin-a-ru $n$. large tree with light colored trunk and strong golden colored wood that is good for posts, (Beng. su-nal-u). <B (4.632)

sin-a-ru-du-ka $n$. a black centipede, less poisonous than sin-a-ru-gitchak. (4.467)

sin-a-ru-git-chak, sin-a-ru-dal-gip-a $n$. a large very poisonous centipede. (4.467)

sin-chi $n$. the hump of cattle. (4.473)

sin-grik-a vt. like each other, want each other, of a man and woman. $(7.722)$

-sin-i num. seven, $(\mathrm{A} \cdot \mathrm{chik}) .(3.3707)$

sin-il-a vt. direct toward; extend a hand, as to a child to help her get up; aim at, of a weapon; push forward, as firewood into a fire. (9.46)

sin-ing-a vi. echo in kal-ak-a sin-ing-a fool around, lead astray, as what a husband does with another woman. (7.26)

sin-ing-a vt. teach, learn: na·-a ang-na Man-di ku-sik-ko sin-inging-a you are teaching me the Mandi language. (8.6)

sin-kip-a vt. turn upside down, of a pot, basket, etc. (9.214)

sin-ku-ak-a vt. want very strongly, esp. of a man for a woman or vice versa. (7.722)

sin-te-a, sin-ti-a vi. hang from a loop, or strap, as from shoulder; hang from a hook, as an umbrella, cane; fly a flag. (9.253)

sin-tik-a, stik-a vt. put on, wear on the head, of hats, glasses; grab in the hair, of burrs; cover, as a rice pot. (6.53)

sin-wak-a vt. want very strongly, of a man for a woman or vice versa. $(7.722)$

sin-a vi. personally cold, of people. (3.675)

sin-chak-a vi. support, prop up, as a banana plant with a post, as the beams of a house. (9.511)

sin-chik-sin'-chik $a d v$. somewhat cool, of people. (3.675)

sin-chip-a vi. cool off, of weather, as when clouds cover the sun. (3.675)

sin·-dik-a vi. be lonely, lack companionship, gloomy, sad. sin·-dik-dik $a d v .(7.743)$

sin-ka-ri $n$. cold season. (2.84)

sing-ga $n$. musical horn made from a buffalo horn and blown without a bamboo blowing tube. $<\mathrm{B}(8.85)$

sing-gi na·-tok $n$. small stinging fish, edible. (4.454) 
sing-gi-ma-ri $n$. a kind of rang (gong). (8.85)

sing-a vi. ask, question, question witnesses at a legal meeting; bark, of dogs. (7.32)

sing-e ni-a vi. ask in order to find out, inquire. (7.32)

sing-kam $n$. a stick used to support a sagging branch of a tree. (5.833)

sing-rik-a $v t$. ask a question of someone who is passing. (7.32)

-sip-, -srip- $a a$. slurp, swirl in the mouth: $\mathbf{k u}$-srip-a swirl water in mouth to rinse it; sal-sip-a suck, as on a straw, have a stuffy nose; ringsip-a slurp, as tea. (0.48)

-sip-, -srip- aa. slurp, swirl in the mouth: ku'-srip-a swirl water in mouth to rinse it; sal-sip-a suck, as on a straw, have a stuffy nose; ringsip-a slurp, as tea. (9.44)

sip-it-sip-it $a d v$. with abundant sweat, perspiration. (6.29)

sip-po-po-a, spo-po-a $v t$. blow up a fire. (9.45)

sip-prong-a, sprong-a vt. blow off dust, dirt; blow rice beer over a sick person during sacrifice; blow up fire; blow out, of matches, hurricane lamp, etc. vi. fly about in air, of dust, powder, etc. (9.45)

sip-prong-chak-a, sprong-chak-a vt. blow through a wa-sing (bamboo tube), to blow up a fire. (9.45)

sit-a vt. grind a tool with sand to sharpen it. (3.666)

sit-a vt. apply, anoint, paint, smear on, as medicine or oil on the skin; paint or scribble on a wall, etc. (9.556)

sit-a, tik ra-ma sit-a $v t$. make a part in the hair. (6.57)

sit-tap-a vi. sticky, of tree sap, of flour mixed with water, etc.; stick like glue. sit-tap-sit-tap $a d v$. (3.668)

sit-tip-a vi. wound up, tangled. (3.718)

-ska dns. of their own kind, that sort of person: tro-ska contemporaries, of the same generation; jat-ska of the same ethnic group. (6.89)

-ska- $a a$. in turn, in return: in-ska-a say in turn, reply. (0.43)

ska-pong, sak-a-pong $n$. top part of an object, as the top part of a drinking glass. (5.87)

skal $n$. an evil person who can kill people by magic, rather than by reciting a chant (mon-tro). (8.51)

skang $p p$. before, (follows dative -na): ang-na skang mi cha-bo eat before me. (1.12)

skang, skang-o $l w$. before, earlier, ago, in earlier days. (2.62)

skang ja-man $a d v$. one after the other, as two people walking. (2.14)

skem-a vi. adv. gritty, texture of sand. skem-skem $a d v$. mixed with sand or dirt, of food that is not good to eat. (3.662)

sket-sket $a d v$. crowded, bumping into each other, filled up, without space, as fruit on a branch, people in a bus. (2.22)

ski-a $n$. teach, learn, (A•chik). (8.6) 
sko, sku $n$. head; end of a rice field, long arm of a rice pounder (teng-ki). (6.22)

sko kam-bi, sku kam-bi $n$. top of the head. (6.22)

sko rik-i, sku rik-i $n$. top of head. (6.22)

sko sa'-dik-a, sku sa'-dik-a vi. headache. (8.94)

sko-ta-ning, sku-ta-ning $n$. brain. (6.27)

sko-tong, sku-tong $n$. headman, leader, (A·chik). (8.12)

skok-skok, skok-ba-kok $a d v$. too quickly, carelessly, especially of eating, drinking. (2.726)

skon-a $v t$. throw into water. (9.513)

skong-a vi. separate from liquid, as cream that rises to the top or dirt that sinks to the bottom. (9.277)

skot-a vi. intermediate between hard and soft, not too hard and not too soft, of fruit, things cooking; not fully ripe; intermediate between tight and loose. skot-skot $a d v$. (3.664)

skrik-a $v t$. remove skin, peel off skin. (4.49)

-sku num. nine, (A•chik). (3.3709)

sku, sko $n$. head, (see sko for compounds). (6.22)

skul, i-skul $n$. school. $<\mathrm{E}(8.6)$

skul-nok, i-skul-nok $n$. school building. $<$ B (5.624)

-so- aa. first, before others, ahead, wait: i-ang-so-a go first, go ahead; seng-so-a wait for; ni-chak-so-a wait for, look out for; cha-so-a eat before someone else; a-gan-so-a announce, tell ahead of time, (cf. cheng- before doing something else). (2.62)

so-a, su-a vi. rot, rotten. (4.696)

so-bok, te'-rik so-bok $n$. banana bud or flower. (4.676)

so-brok-a, su-brok-a vi. completely rotten, not good to eat. (4.696)

so-dap-gek-gek, su-dap-gek-gek $a d v$. very dirty, rotten, unwashed. (3.716)

so-dap-ki-ki, su-dap-ki-ki $a d v$. dirty, unwashed. (3.716)

so-dap-prong-prong, su-dap-prong-prong, so-dap-prang-prang adv. dirty, unwashed. (3.716)

so-drak-sa-drak adv. tattered, torn. (9.292)

so-ek-a, su-ek-a vi. rash between toes: $\mathbf{j a}^{\cdot}-\mathbf{s i}$ so-ek-a the toes get a rash. (8.953)

so-eng-a, su-eng-a vi. smell badly, stink, (A•chik: se-eng-a). (3.688)

so-eng-kal-ting-a, su-eng-kal-ting-a, su-eng-a-kal-ting-a vi. rotten, smelly, of food or of someone who does not wash. (3.688)

so-gon, su-gon $n$. vulture. $<\mathrm{B}(4.43)$

so-hor $n$. city. $<\mathrm{B}(8.22)$

so-man, so-man-so-man adv. level, even, smooth; equal in age, size, quality, etc. $<$ B (2.311) 
so-mas, so-maj $n$. society, social group. $<\mathrm{B}(8.48)$

so-mi-ti $n$. organization, committee, club. $<\mathrm{B}(8.48)$

so-mo-bai $n$. cooperative: so-mo-bai so-mi-ti cooperative club. $<\mathrm{B}$ (8.48)

so-moi $n$. time. $<\mathrm{B}(2.51)$

So-ni-bal $n$. Saturday. $<\mathrm{B}(2.817)$

so-pok-a, su-pok-a vi. starting to turn rotten. so-pok-so-pok adv. (4.696)

so-prot-a, su-prot-a vi. rotten, soft and old, full of holes, as wood when eaten by insects. (4.696)

so-ras $n$. a stringed musical instrument. $<\mathrm{B}(8.85)$

so-rok $n$. road, highway. <B (5.646)

so-si-a vi. wet. (9.272)

so-sra, so-sa, su-sra, su-sa, su-su-ra $n$. cucumber. (5.23)

so•-a vi. echo in bo:-a so•-a fool around, joke. (7.25)

so•-a, su·-a vt. burn, ignite, light fire, candle, etc.; feed fire with firewood; singe a pig over fire to remove hair. (5.424)

so-chip-a vt. burn completely. (5.424)

so-e net-a vt. light, shine, as a hurricane lamp, oil lamp. (5.424)

so-ga-det-a, so-gat-a $v t$. feed firewood into fire. (5.424)

so-krep-a, su•-krep-a vt. cremate, burn a dead body. (6.79)

so'-mi-si so'-ma-sa $a d v$. how the body is without bathing. (6.58)

so·-ot-a vt. kill. (6.79)

so-sim-prak-a vt. blacken with fire, scorch, as when a butchered pig is scorched to clean it and remove its hair. (5.424)

so-so-a, su•-su-a vi. painful, from chilies that burn when tasted, or esp. for burning in a wound. (3.682)

so-so-jeng-jeng, su-su-jeng-jeng $a d v$. going from this to that without finishing anything, having too much work. (8.433)

so'-srom-a vt. cook in a tied up banana leaf. (5.46)

so-tep-a $v t$. cook in a folded banana leaf. (5.46)

soi-tan $n$. satan, devil. <B (8.54)

sok $n$. breast, man's nipple; mother's milk, animal's milk when drunk by a baby animal. (6.245)

sok cha-mit-ing $a d v$. while nursing, a stage of child development. (6.78) sok-a vi. arrive, reach. -sok- $a a$. arrive, reach: rang-sok-a get one's breath; am-sok-a confident, able to manage; chu•-sok-a enough, enough to reach; mik-sok-a hope, suspect. (9.313)

sok-bit-chi $n$. breast milk, mother's milk, (lit. breast-juice). (6.29)

sok-chon $n$. tree with white sap and $12 \mathrm{~cm}$. whorled leaves. (4.632)

sok-dik-a vi. arrive unexpectedly, without warning, at the wrong time. $(9.313)$ 
sok-kit-e, sok-kot-e $n$. nipple. (6.245)

sok-me, sok-mi $n$. woman's breast. (6.245)

sok-me cha-mit-ing $a d v$. during the time of small growing breasts, a stage of maturation. (6.78)

sok-me ja-ping $n$. breasts and thighs, the parts that tempt men. (6.245)

sok-pak $n$. the last child, last baby, youngest of a set of siblings. (6.64)

sok-sin $n$. the next child, next youngest, (same as ja-sin). (6.64)

sok-tik-a vi. fully arrive; often negative: sok-tik-ja not quite arrive, not quite reach. (9.313)

sol-di $n$. cold, sniffles. $<$ B $(8.92)$

sol-sol-a $n$. silkworm. (4.467)

sol-srim $n$. medium complexion within the local range, neither very light, nor very dark in complexion. (3.539)

sol-a vt. carve, cut off pieces, esp. from the surface, to reduce the size of a wooden piece; scrape off mud from the walls of a new house to make them smooth; remove bark, trim, whittle; skin an animal. (9.573)

sol-ok-a vt. carve, as a memorial post; shape and smooth the surface, more delicate than sol-a. (9.573)

sol-srok-a vt. make smooth by cutting or carving a little bit from the surface; polish. (9.573)

Som-bal $n$. Monday. <B (2.812)

som-bol $n$. means of support, savings, provisions, supplies; an old man's stick. (8.427)

som-pot-i, soi-som-pot-i, som-pot $n$. property. $<\mathrm{B}(8.423)$

son-choi, son-choi so-mo-bai $n$. the type of club that collects savings money. $<$ B (8.48)

song $n$. village. song- $c l s$ classifier for villages. (8.22)

song-a vt. set up, as post. (9.571)

song-chi $n$. fence post; the vertical bamboos used between the wooden posts of an old fashioned bamboo house. (5.642)

song-dong-a $v t$. settle a village. (8.22)

song-jot-a $v t$. replace a post. (9.571)

song-nok $n$. settlement, the entire village area. (8.22)

song-pi nok-pi $n$. the whole village. (8.22)

Song-sa-rek $n$. the A-chik name of the traditional religion of the Garos, (Mandi: Sang-sa-rek). (8.51)

song-a vt. cook; brew rice beer. (5.46)

song-min-ek-a vt. cook to softness. (5.46)

sop $n$. all. $<$ B (3.343)

sop-ta $n$. week. cls. classifier for weeks: sop-ta-prak, sop-ta-rik-it, sop-ta-jring every week, each week; sop-ta-gin-i two weeks. $<$ B (2.53)

sor-dar $n$. agent of a village head, speaker at village bi-char (legal meetings). $<\mathrm{B}(8.12)$ 
sot- num. prefix for tens in counting, as sot-bri forty, sot-chet eighty, etc., (A·chik). (3.3710)

sot-a vt. chop, cut firewood. (9.573)

sot-ku-rang-a $v t$. chop lengthwise. (9.573)

sot-ni-o, sot-mi-o lw. day after tomorrow. (2.65)

sot-tang-so $n$. a land measure, 1/100 acre, 1/30 pa-ki, (same as ko-ra or dis-mel). $<\mathrm{B}(5.12)$

sot-tong-a vt. chop across, into lengths, chop into two parts, of a log, stick, etc.; cut clear through. (9.573)

spak-a vi. a bad puckering taste, as that of areca nut when eaten alone, like some very unripe fruits. (3.682)

spi, sep-pi $n$. warrior's shield. (5.845)

spin $n$. sesame. (5.405)

spo-a, spu-a vt. blow, as on a fire, either to make it burn, or to extinguish a flame. (9.45)

spok $n$. spoke of a wheel. $<\mathrm{E}(5.872)$

spok-a vt. insert one cylindrical thing into another, as a small bamboo into the end of a larger one to make a longer pole, stack glasses, put on the cap of a pen, plug up as with a cork in a bottle. (9.571)

spom-a $v t$. hold inside the mouth without chewing, as water, betel, etc. (5.472)

spru, spu-ru $n$. small edible snail, (A·pal, A•chik), (same as chot-chru). (4.458)

Sra-bon $n$. a Bengali month that overlaps July and August. <B (2.824) sra-rit-tep $a d v$. abundantly, of speaking quickly and fluently, of hail. (7.14)

sra-sra, sra-e-sra-e $a d v$. in large numbers, fall in large amounts, as rice grain. (3.342)

srak-a vt. lick. srak-e-srak-e $a d v .(9.44)$

srak-srak, mik-a srak-srak adv. intermediate between rough and smooth, not slippery. (3.662)

sram-a vi. crumbly, brittle, easily breakable, the texture of dry dirt, dried leaves, fried foods, puffed rice. (3.668)

-srang- $a a$. completely: i-ang-srang-a completely gone, gone for good. $(3.342)$

srang-a vi. clear up, be clear, of water, (same as grik-a); of clouds, (same as krang-a); of hearing, of a headache: gi-sik srang-a clear up, of the mind, leaving no worries or problems. ku-pa srang-a vi. clear in language, honest. srang-e $a d v$. clearly, completely, of speaking, seeing, hearing, understanding: na-chil srang-e kin-a ears hear clearly; srange hai-ku-ja don't yet understand completely, not yet clear. (3.714)

srang-srang $a d v$. sharp, of a knife; fluently, clear, clearly, exactly, as speaking clearly. (3.666) 
srap-a vi. catch up with, find someone; often in negative: srap-ja cannot catch up. -srap- aa. catch up with: kat-srap-a run and catch up with; rim-srap-a catch, as a chicken, a thrown object; in-srap-a catch the points of a conversation, have a dialogue. srap-srap, srap-e-srap-e $a d v$. going together, follow one another closely, of people or animals. (9.328)

srap-nik-grik-a vi. catch up with and see each other. (9.328)

sre, sri, si-ri $n$. tongue. (6.22)

sre ja•ting, sri ja•ting $n$. back of the tongue, base of the tongue. (6.22)

sre-a, sri-a vt. exchange, trade. (8.444)

sre-bak, sri-bak $n$. back of the tongue; the part of a traditional man's loin-cloth that hangs down in front, front flap. (6.22)

sre-grik-a, sri-grik-a vt. exchange, trade. (8.444)

sreng- cls. classifier for single bunches of thatch. (1.34)

-sret- aa. slip away: cha'-sret-a slip from the mouth; rim•-sret-a slip from the hand. (9.54)

sri, si-ri, sre $n$. tongue, (see sre for compounds). (6.22)

sri-a $v t$. to slice lengthwise. sri-sri $a d v$. in lengthwise slices: sri-sri dak-e rat-a cut lengthwise. sri- cls. classifier for lengthwise slices, of papaya, etc. (9.573)

sri-a, sre-a vt. exchange, trade. (8.444)

sri-sra $a d v$. in large amounts, of rain, birds or flying insects, earth lumps. $(3.342)$

srik-a vt. shave, cut with a razor; scrape or clean the surface, as with an at-te (bamboo knife) or hoe; scrape in order to make smooth, of bamboo strips, of the mud of new house walls; smooth with a plane. (9.573)

sring-a vt. string up, hang, as a mosquito net, a rope. (9.65)

-srip-, -sip- aa. slurp, swirl in the mouth: ku-srip-a swirl water in mouth to rinse it; sal-srip-a suck, as on a straw, have a stuffy nose; ring-srip-a slurp, as tea. (0.48)

-srip-, -sip- aa. slurp, swirl in the mouth: ku-srip-a swirl water in mouth to rinse it; sal-srip-a suck, as on a straw, have a stuffy nose; ring-srip-a slurp, as tea. (9.44)

srip-srip, srip-e-srip-e $a d v$. sloppily, as when eating a liquid or of wet food. (5.472)

-sro, -sru ns. each, generally for distributing things: pil-sa-sro one coin each; tang-ka pat-gin-i-sro two takas each; mi rang-tal-sa-sro one plate of rice each; kap-sa-sro ron-bo give one cup each. (3.35)

sro-a, sru-a, jin-ang sro-a vt. borrow at interest, as rice, money. (8.446)

srok, srok-srok, srok-srok-e, srok-e-srok-e, sruk, sruk-sruk adv. quietly, secretly. (3.627)

-srok- aa. lightly, loosely, gently, partially, incompletely: dok-srok-a hit lightly; ha-bu-srok-a bathe quickly or partially; a-gan-srok-a tell 
partially, not everything; ka-srok-a tie loosely; ken--srok-a comb slowly and carefully. (3.64)

srok-a vi. recede, of water from a high point. (9.273)

srom-a $v t$. wrap in a banana leaf so that the sides are brought up to form a mouth that is then tied shut, usually for curry. (5.453)

srong-a vi. straight, of roads, of a line of trees, etc.; straight speaking, truthfully. (2.318)

srot-a vi. slip, slide. srot-srot $a d v$. in a sliding manner: srot-srot dak-e i-ang-a slide along, slip while going. -srot- $a a$. slip: rim•-srot-a let slip with hand; ga·-srot-a slip with the feet. (9.326)

sru-a vi. overflow, boil over. -sru- $a a$. overflow: git-u-sru-a boil over; chi-sru-sru overflow, of water from a pot; $\mathbf{k i} \cdot-\mathbf{s r u}-\mathbf{a}$ have diarrhea; $\mathbf{k i} \cdot-\mathbf{s i}-$ sru-a fart a lot. (5.46)

sru-sru $a d v$. gradually, slowly, esp. of leaking: chi sru-sru ong•-kating-a water is coming out slowly; as something might leak as one walks along the road. (2.724)

sten $n$. bottom corner of a basket on which the basket rests, bicycle stand. $<\mathrm{E}(5.854)$

steng $n$. a wild tree-climbing vine with a large edible tuberous root that, if eaten, is filling. (4.655)

sti-a, sit-ti-a vt. hang from a strap or hook, (same as sin-te-a). (9.253) stik-a, kin-i stik-a vi. tangled, of hair, vines; uncombed. (6.58)

stik-a, sin-tik-a vt. put on, wear on head, of hats, glasses; grab in hair, of burrs; cover, as a rice pot. (6.53)

stil $n$. hail. (4.329)

stil bal-wa dak-a vph. hail with wind. (4.329)

stip-at, stip-et, dip-at $p p$. as much as. (1.17)

stu-a vt. spit. (9.44)

su-a, so-a vi. rotten, (see so- for compounds). (4.696)

su-a vt. peck, peck at, of birds. (9.571)

su-a-biin, su-a-piin $n$. soybean. <E (5.23)

su-al-a $v t$. divide, apportion, share, esp. for property and goods other than money. (8.427)

su-ang-su-ang $a d v$. see or hear dimly, in the distance. (3.54)

su-bu $n$. urine, (A·chik). (9.49)

su-del-a, git-ok su-del-a vi. having a hoarse voice. (3.624)

su-grik-a vt. peck at each other, of birds. (4.487)

su-i ron-a, su-i sik-a vph. a piercing whistle made by blowing over a bent finger; louder than $\mathbf{k u} \cdot$-sim-ok-a. (7.29)

su-kul-ot-a vt. pecking, by birds. (4.487)

su-na $n$. gold. $<\mathrm{B}(5.88)$

su-na-muk dail $n$. a variety of dahl, the only kind grown in Modhupur. $<\mathrm{B}(5.23)$ 
su-rek-su-chek $a d v$. with disgust. (7.744)

su-ri $n$. large tree with good wood, a few vertical cuts in the bark, and fruit that has a nut-like core, (Beng. boi-ra). (4.632)

su-ri-bit-e $n$. fruit of the wild su-ri tree, that has a nut like core. (5.22)

su-sa-a vi. compete, race: kat-su-sa-a race by running. (8.81)

su-sim-pok-a vt. set down firmly, often angrily, as an object, or a child who is fussing. (9.511)

su-song-a, su-song-e ni-a vt. measure the depth of water. (9.66)

su-stim $n$. tree with shaggy reddish bark, and $30 \mathrm{~cm}$. pods that are ripe in April and eaten by cows but rarely by people, (Beng. chu-ka-ko-la). (4.632)

-su-su- $a a$. in part, incomplete: $\mathbf{r i m} \cdot \mathbf{- s u - s u - a}$ do in part, not all; watsu-su-a weave only part (of a basket). (3.347)

su-tek-a vi. fall down frontward, head first, hit head against the ground. (9.326)

su-tet-a, su-tet-e cha-a vt. peck like a bird, peck and pick up or carry off. (4.487)

su-wak-a vt. scrape up and throw dirt, scoop. (9.576)

-su•- aa. wash: jak-su-a wash hands; mik-su-a wash face; su•-gal-a wash things, clothes, dishes. (6.57)

su·-a vt. pierce, stab, spear; inject medicine; cut open; pound rice, husk; pound into a tube; bite, of snakes. (9.571)

su·-a, so•-a vt. burn, (see so•- for compounds). (5.424)

su·-brok-a vt. to spear, stab with a spear, pierce. (9.571)

su-chek-chek-a vi. well and thoroughly pounded and clean, of rice; poke here and there with a tool. (5.451)

su-chip-a vt. pierce into blindly without seeing. (9.571)

su-dep-a vt. pound, as with a hammer; pound into a bamboo tube; pound a finger in a rice pounder. (9.553)

su-drak-a $v t$. poke and tear. (9.571)

su•-gal-a vt. wash things, such as clothes or dishes. (6.57)

su·-gel-gel-a $v t$. leave rice threshing unfinished, without all the poundings. (5.451)

su-gip-a, ang-su $n$. grandchild. (6.617)

su-kang $n$. cactus-like plant with long sharp leaves on straight but leaning meter high stalk. (4.656)

su·-krep-a vt. pound, crush, as fingers getting pounded by mistake in a rice pounder. $(9.578)$

su·-mik-brang-a $v t$. stab without seeing what is stabbed. (9.571)

su'-mit-cheng $n$. large tree with very thorny trunk and vaguely sumaclike leaves that are used in cooking, and oil is made from the fruit. (4.632) su-prot-a $v t$. pierce, make a hole, esp. in order to get out a thorn or pus. $(9.571)$ 
su•-prot-a, bu-su su·-prot-a $v t$. pierce and make a hole, esp. to get out a thorn or pus. (8.99)

su·-pru-a $v t$. pick or pierce an infection or a wound to extract puss or to work out a thorn or splinter. (8.99)

su'-rik-rik-et-a vt. pound or stab steadily, as when pounding rice; add lots of wood to a fire. (9.571)

$\mathbf{s u} \cdot \mathbf{r u - r u} a d v$. echo in jot-ru-ru su•-ru-ru poke into. (9.571)

su-sam $n$. small biting fly, gnat. (4.462)

su·-sik-a vt. pound into, fill up, as dried fish into a bamboo tube, mud into cracks in a house wall. (9.571)

su'-srok-a $v t$. third and final pounding of rice in a rice pounder, to whiten it. $(5.451)$

$\mathbf{s u}-\mathbf{s u}-\mathbf{s a} \cdot \mathbf{s a} a d v$. dirty of the body, unbathed. (6.58)

$\mathbf{s u} \cdot \mathbf{s u}-\mathbf{s a} \cdot \mathbf{s a} a d v$. restless, wiggly, not stay quiet. (9.324)

su-tat-a $v t$. poke with pointed object; attack with spears; poke with fist. (9.571)

su-ting-dat-a vt. strike, poke, with elbow, knee, hand or stick. (9.571)

sui-tar $n$. sweater. <E (6.521)

suk-ki, sik-ki $n$. four anna coin, quarter-taka. <B (8.447)

suk-ki-so-ra $n$. necklace made from coins. (6.563)

Suk-ro-bal $n$. Friday. $<$ B (2.816)

sur-sal-e bol-a vph. speak slowly, in a stretched out style, comparable to the streched style used in some singing. (7.15)

sut, sud $n$. interest on a loan. $<\mathrm{B}(8.442)$

sut cha-a vph. take interest, charge interest like a money lender. $<\mathrm{B}$ (8.446)

sut-kut $n$. fine clothes. $<\mathrm{E}(6.527)$

sut-re-han $n$. mortgage, give land to another person to use in return for a loan of money, with the land to be reclaimed only when the loan is repaid. $<\mathrm{B}(8.425)$

ta-ka n. taka, Bangladeshi unit of currency. $<$ B (8.447)

ta-kri, ta-kri dail $n$. white dahl, a variety of dahl. $<\mathrm{B}(5.23)$

ta-mak, ta-mak-ku n. tobacco. <B (5.429)

ta-muk $n$. tobacco; the tobacco that is used in a water pipe, (same as mol-a). $<$ B (5.429)

ta-na $n$. police district, police station. $<\mathrm{B}(8.22)$

ta-ning, sko-ta-ning, sku-ta-ning $n$. brain. (6.27)

ta-ra-ta-ri $a d v$. quickly, early. <B (2.726)

ta-ri-a vt. prepare. vi. get ready. (9.13)

ta-riik $n$. date, day of the month. $<\mathrm{B}(2.51)$

ta-rim-e $a d v$. along with, together with. (2.11)

ta-ru $n$. baby's soft spot, fontanel. (6.22) 
ta-wal $n$. towel. $<\mathrm{E}(6.521)$

ta'- $c p$. prefix for varieties of tubers: ta・-mil-ang sweet potatoes; ta・-bolchu manioc; ta-ring arum. (0.17)

ta'-a $n$. any of several edible tubers. (5.27)

ta-ak $n$. a large inedible wild tuber. (4.653)

ta-bi-dil $n$. vine of a tuber plant. (4.670)

ta-bi-rot $n$. white potato, (same as al-u gu-ta). (5.27)

ta-bit-chi-ri $n$. the top part of a tuber that is not eaten but that can be planted. (4.677)

ta'-bol-chu, ta'-bil-chu $n$. manioc, cassava. (5.27)

ta-dam-bu $n$. a large-leafed cultivated tuber. (5.27)

ta-ding-ding, ta•-ding-ta'-rot $a d v$. in a line, very straight, as a line of areca palms, of a road; very smooth; straight speaking. (2.318)

ta-git-chak $n$. an edible wild tuber. (4.653)

ta-ja $n$. an edible wild tuber. (4.653)

ta--jong $n$. a large bulbous cultivated tuber. (5.27)

ta-ma $n$. a large edible tuber. (5.27)

ta'-man-de $n$. a variety of cultivated tuber. (5.27)

ta'-mat-chi $n$. a cultivated vine with edible root the size of a fist or a bit larger; said to be the favored tuber of the Mat-chi people, a sub-group of Garos who have never been seen, but who are reported never to wash, to eat cats and dogs, and perhaps to live in the Garo Hills. (5.27)

ta・-mi-sel $n$. an edible wild jungle tuber. (4.653)

ta'-mil-ang $n$. sweet potato. (5.27)

ta'-nu $n$. a large inedible wild tuber. (4.653)

ta-rak-a vi. quick, fast, as of speaking; strong. (2.726)

ta-ring $n$. huge-leafed tuber, arum. (5.27)

ta-rip-u $n$. vine with pea-like pods, and an edible white fleshed tuber. $(5.27)$

ta-ru-ri $n$. a kind of cultivated tuber. (5.27)

ta'-sko, ta:-sku $n$. the top part of a tuber that is not eaten but that can be planted, cutting, (lit. tuber-head). (4.677)

ta-tu-rak $n$. a small round cultivated tuber. (5.27)

ta--wek $n$. cooked ta--ring, cooked arum. (5.409)

-tai- $a a$. again: grap-tai-a cry again, $(\mathrm{A} \cdot \mathrm{chik}) .(2.71)$

tai-ar $n$. tire. $<\mathrm{E}(5.872)$

tak-a $v t$. carve, as the notches in a log to be used as steps, as a memorial post; hit, strike, poke, tap, as one taps the smallest kind of drum; the tap of a woodpecker. (9.573)

tak-tak den-a vph. make small chops, carve in several places, as the top of a memorial post. (9.573)

tak-tak-a, tak-tak-a rak-rak-a vi. make a tapping sound, as the sound of beating a finger on the table, of typing. (3.624) 
tak-tuk, tuk-tak $n$. odd jobs; a bit from here, a bit from there, as when looking for things. (8.433)

tal $n$. kind of palm tree with fan-like leaves, the fruit of which is cooked but not eaten raw. $<$ B $(4.632)$

tal-a $n$. lock. $<\mathrm{B}(5.82)$

tal-a vi. be clean, clear. (3.714)

tal-a tek-a $v p h$. closed with a lock. (9.61)

tal-i $n$. rice paddy, a single wet rice field within a dike. tal-i- cls. for rice fields. (5.114)

tal-tal-a vi. make the noise of falling water. (3.624)

tam-pi $n$. fly. (4.462)

tam-pi-a-mak $n$. a small fly that bites cows and sometimes people. $(4.462)$

tam-pi-rong-reng, rong-reng $n$. big blue fly that eats dung, horsefly. (4.462)

tam-pi-sim-ik $n$. tiny non-biting fly that comes out in swarms, gnat. (4.462)

tam--bik, tam•-bik-bik adv. short. (2.215)

tam-bing-a vi. round, like a football, wheel, ankle bones, end of a flash light, sun, full moon, looped bamboo strip, egg, water pot. tam•-bing-

bing, tam-bing-jret-jret adv. (2.313)

-tang dns. a suffix used with kinship terms that makes them terms of reference rather than as terms of address: am-bi-tang grandmother; gri-tang nephew. (6.64)

-tang ns. own, self, selves: song-tang own village; nok-tang own house. (0.11)

tang-a vi. live, alive. (6.77)

tang-cha-a vi. come to life. (6.77)

tang-cha-pil-a, tang-pil-a vi. come to life again. (6.77)

tang-cha-rik-rik-a vi. come to life again. (6.77)

tang-et-a $v t$. care for, save, (lit. cause to live). (6.77)

tang-ka $n$. money, especially paper money, $(\mathrm{A} \cdot$ pal, A•chik). <B (8.447)

tang-si-ru-ru, tang-sik-ru-ru adv. fine bright color. (3.539)

tang-sik-a vi. green colored, greenish; not yet dead, of plants. (3.534)

tang--a vi. fly through the air, of things thrown, kicked, etc., but not so far as til-a. (9.216)

tang-a, dang•-a vt. fit, big enough to fit, fit, of clothes, a door, a bracelet: sko tang-a fits for the head, right sized for a head, the head passes through. (2.22)

tang-nap-a vi. be reborn; change groups, enter a new group, as when a son-in-law joins his wife's family; change ethnicity, as when a Mandi becomes a Bengali or vice versa; get into, fit into a hole, enter into, as a 
bug in the eye, an insect into fire). ec. echo in at-chi-a tang-nap-a be born. (6.77)

tang-pil-dat-a vi. jump back, jump away in fright, as when seeing a snake. (7.763)

tang-pret-a vi. exploding noise, of bamboo bursting, of a bomb, a firecracker. (3.628)

tang-rong-rang-a vi. fit easily into or through, as through a sieve; loosely fitting, as bracelets, sometimes used for bigger things than gip-ua which is used only for fine things. (2.22)

tap $a d v$. quickly, used in commands: tap $\mathbf{i} \cdot$-ba come quickly!; tap ra-a bring it right away! (2.726)

tap teng-re $a d v$. fast, quickly, right away. (2.726)

tap-a vt. dot with glue, make a thumbprint. -tap- aa. stick to, apply, attach: ma-tap-a paste, stick, paste up; apply to the skin, as medicine; sik-tap-a sew on a patch; ga-ak-tap-a fall onto; pa-tap-a glue to, stick to, attach. (9.63)

tap-a, tap-e ni-a $v t$. taste a bit in order to sample, test by licking from a finger or palm. (3.682)

tap-jang-jang adv. clear, of liquid. (3.714)

tap-ma-tap-ma, tap-pu-tap-pu adv. come out, of the rash from the touch of a mang-tip worm. (8.94)

tap-ol-a $n$. blister. (8.955)

tap-pa-reng-reng $a d v$. sharp, sharply pointed, as a needle, thorn, etc. $(2.312)$

tap-ping-a $v t$. make a noise by splashing water. (9.277)

tap-pu-tap-pu $a d v$. come out, of the rash from the touch of a mang-tip worm. (8.94)

tap-seng-tap-seng $a d v$. a little sour but good tasting. (3.682)

tap-skot-a $v t$. not hold on to, let slip from the hand, let go. (9.54)

tap-srang-tap-srang $a d v$. clearly, of speaking, explaining. (7.34)

tap-tu-a $v t$. forget. (7.45)

tar-por, tar-pore, ta-ba-de, tar-ba-de, tar-ba-de-se, ta-har, tar conj. then, after that. $<\mathrm{B}(1.4)$

-tat- $a a$. forcefully: dok-tat-a hit forcefully; sik-tat-a grab by force, as sons-in-law in the old days; ni-tat-a stare. (7.56)

tat-a vt. prick, pick at; poke something into infected flesh; hurt with thorns or spikes but without poison, as by ging-gi and teng-ra fish. (8.99)

tat-chi $n$. echo in chat-chi tat-chi kinsmen. (6.63)

tat-nok $n$. weaving place. (5.624)

te int. yes, indicates generalized agreement rather than specific confirmation of a question. (1.24) 
-te pvs. doing and doing, continuing. (0.211)

te-bil $n$. table. $<\mathrm{E}(5.82)$

te-ta $n$. fish catching fork made of metal, shorter than a kus. (5.843)

te•-, ti- $c p$. prefix for various fruits: te•-rik banana; te-brong jackfruit; te'-ga-chu mango; bi-te, bi-ti fruit. (5.22)

te-brong, ti-brong $n$. jackfruit. (5.22)

te•-ga-chu, ti-ga-chu $n$. mango. (5.22)

te-kring, ti-kring $n$. tree with willow-like leaves and red sour fruit that is ripe in July, and wood that is good for house posts, (Beng. ne-ur). (4.632)

te-mat-chu, ti-mat-chu $n$. lime, (same as le-bu). (5.22)

te-na-rang, ti-na-rang $n$. tree with a lemon-sized edible fruit that looks like a gourd but is soft, and that is bitter when unripe, (Beng. pa-hai-ra bo-roi). (4.632)

te-pak-krong $n$. big bush or small thorny tree up to about 3 meters tall, with little round green fruit, a weed, not eaten. (4.64)

te'-rak-rak, ti-rak-rak $a d v$. suitable size, not too big, not too small. $(2.22)$

te-rik, ti-rik $n$. banana. (5.22)

te-rik so-bak $n$. the large edible bud of a banana tree. (5.22)

te•-rik-gal-wang $n$. pencil-sized vine with $10 \mathrm{~cm}$. oval leaves, used to make the rims and vertical corner pieces of baskets, and as a substitute for cane, (Beng. ba-no-rel). (4.655)

te•-seng-ki, ti·-seng-ki $n$. tree with $2 \mathrm{~cm}$. round leaf, edible red sour fruit that is a bit sweet when ripe in May, (same as kang·-ka-ri). (4.632)

te-seng-ki-mak-bil, ti-seng-ki-mak-bil $n$. tree with pointed oval leaves and edible fruit ripe in May, (Beng. jong-la bo-roi). (4.632)

tek-a vt. close, shut, lock door; fasten with a button; shut up in two bamboo halves; close a bottle, etc. with a cap. (9.61)

tek-ki-a vi. lack something, without money or rice; in need. <B (7.726)

tek-ki-a pal-ing-a $v t$. sell when in need, as when selling crops cheaply before they are ripe, selling cattle when needing rice. (8.444)

tek-kra, tek-la, ti-kra, ti-kil-a $n$. little plug of earth placed in the bottom of a kol-ki (pottery pipe bowl) to keep the tobacco from falling through. <B (5.429)

tek-rak-a vi. crumbly, like leaves. vi echo in rong-kal-a tek-rak-a joke, tease, fool around. (9.292)

tek-sram-tek-sram adv. crumbly, brittle. (3.668)

tek-tek $a d v$. enough, sufficient: bil-si-sa-na sok-o-na tek-tek sufficient to reach (last) for one year. (3.341)

tek-tek-a vi. make a non-ringing sound when struck. (3.624)

tel $n$. oil. $<$ B (5.405) 
teng-gil $a d v$. very, very much, excessive. (3.342)

teng-ki $n$. footoperated rice pounder. $<\mathrm{B}(5.835)$

teng-re, teng-ri, teng-re-teng-re, teng-ri-teng-ri adv. early, soon, fast, quickly. (2.726)

teng-teng-a vi. make a ringing sound when struck, squeaking sound made by squirrels. (3.624)

teng- $c l s$. classifier for the steps of a ladder. (1.38)

teng•-a vi. shine. (3.556)

teng-a vt. carve, as a memorial post, or the notches in a log to be used as steps. (9.573)

teng•-kap teng•-kap $a d v$. quickly, briefly, immediately, at once, right away. (2.53)

tep-a $v t$. wrap up as in folded banana leaves, with wrapper folded over, (same as cho-gep-a). -tep- aa.: cho-tep-a wrap in folded banana leaves; so-tep-a cook in folded banana leaves. (5.453)

tep-tep-a vi. shiver from cold or fright. (9.322)

-tet- aa. break off: ak-tet-a pluck off, of flowers, leaves, etc.; sal-tet-a pull apart; chik-tet-a bite off. (0.44)

tet-a vt. break, of string or other linear things. -tet- aa. break off: ak-tet-a pluck off, of flowers, leaves, etc.; sal-tet-a break by pulling, as string; chik-tet-a bite off a piece. kil-ding tet-a vt. a ceremony in which a thread is strung from a sick person to outside the house. When the thread is broken the mit-e (spirit) that is causing the disease can no longer come to the sick person. (9.284)

tet-tip-tip-a $v t$. break, of string and other linear things. (9.284)

tet-tong-a vt. break apart, of string and linear things. (9.284)

ti-char $n$. teacher. $<\mathrm{E}(8.6)$

ti-kra, ti-kil-a, tek-la, tek-kra $n$. little plug of earth set in the bottom of a kol-ki (pottery pipe bowl) to keep tobacco from falling through. $<\mathrm{B}$ (5.429)

ti-up-wel, tup-wel $n$. tube well with a pump that has standard old style up and down handle. $<\mathrm{E}(5.642)$

ti- cls. classifier for pots, water jugs (gim-bi), etc. (1.38)

ti-, te- $c p$. category prefix for fruits, (see te'- for compounds). (5.22)

ti-a-kol $n$. depression at the back of the head, at the top of neck. (6.241)

ti-ik-a vi. hiccup. (9.44)

tik $n$. louse, head louse. (4.468)

tik ra-ma $n$. part in the hair, (lit. louse path). (6.25)

tik-a vi. enough, as of food being given out to a crowd: cha tik-jok tea is sufficient. (3.341)

tik-a, jang-gil tik-a vi. turn one's back; with one's back toward something. (9.349) 
tik-grik-a vi. back to back. (9.349)

tik-kin-i $n$. hair whorl. <B (6.25)

tik-ot-a vt. flick something with a finger; pinch off a bit with fingers or nails; scratch with one nail or claw in order to hurt, by people or animals. (9.531)

tik-tak-a $v t$. tap, as with the tip of a finger. (9.46)

tik-tik-a, dik-dik-a vt. cluck, sound made by hen to call her chicks. (4.476)

til-e-reng $v i$. call of do•-til-eng bird. (4.476)

til-a vi. fly or move as a result of a push or throw, further than tang•-a; rise up, as a spark. (9.216)

til-ak-til·-ak chrok-a vph. jump up and down in joy and excitement. (9.318)

til-nap-a vi. fly onto a person, fly into the eye. (9.216)

til--til•-a vi. shake with cold or fear, shiver. (9.322)

-tim- aa. stealthily, attentively, continuously: dong-tim-a wait, stay and guard, watch over; ni-tim-a watch attentively, continuously; si-kaltim-a hunt stealthily; kat-tim-a run off and hide; kin-a-tim-a listen secretively; chok-tim-a write regularly, constantly; $\mathbf{i}$-ang-tim-a go all the time. (0.48)

tim- cls. classifier for sections (git-tim) of a village. (1.37)

tim-ang-a vi. step into a hole; get into trouble by doing something wrong. $(9.326)$

tim-bing-tim-bang adv. having equal height and width, as a square. $(2.313)$

tim-bong, tim-bong-tim-bong, tim-bing-tim-bong $a d v$. wide, of rice fields. (2.213)

tim-e tim-e $a d v$. secretly, in hiding. -tim- $a a$. stealthily, attentively, continuously: dong-tim-a wait, stay and guard, watch over; ni-tim-a watch attentively, continuously; si-kal-tim-a hunt stealthily; kat-tima run off and hide; kin-a-tim-a listen secretively; chok-tim-a write regularly, constantly; $\mathbf{i}$-ang-tim-a go all the time. (9.344)

tim-ing-tim-ang $a d v$. unevenly, of a road with high and low spots; speak unevenly with many pauses. (2.319)

tim-ing-tim-ang $a d v$. this way and that. (2.14)

tim-u-a, tim-a vi. hide, wait; tim-u-e ni-a watch in secret. (9.344)

tim- cls. classifier for groups of animals, people, houses, small mounds of earth, etc. (1.35)

tim-ka-a vi. gather, gather together; be together, of a number of people. $(9.535)$

tim-tim-a vi. make a loud noise, of thunder, of rice being ground in a mill, of a tube well, motor, train. tim•-tim adv.: tim•-tim mik-u-a make a sound of thunder, etc. (3.624) 
tin- cls. classifier for chunks, pieces of things, as pieces of meat, tubers, lumps of earth, etc. (1.37)

tin-chon-a vi. small, of chunky things, of tubers, meat, lumps of dirt, etc. $(2.211)$

tin-dal-a vi. big, of chunky things, of tubers, heads, etc. (2.211)

tin-dik-a vi. gather, come together in a crowd. (9.535)

tin-sok $n$. kidney, heart. (6.27)

ting-a $v t$. poke people or things, as with hand or elbows. -ting- $a a$. strike, bump into: nang-ting-grik-a bump into each other; ga-ting-a kick, push with the foot; dok-ting-a strike, knock. (9.552)

ting-a $v t$. separate by spreading a barrier, as to line the bottom of a bird basket with leaves or straw to catch bird droppings or to receive eggs; stretch skin over drum end. (9.536)

ting-a, ja•-sku ting-a vi. kneel. (9.349)

ting-chang-a $v t$. enclose, separate off, as with leaves or cloth for layers in a basket or with a wall or a fence. ting-chang- cls. classifier for sections of a building divided by a barrier: ting-chang-gin-i two sections of a building, two rooms. (9.536)

-ting-dat- aa. bump, strike: su-ting-dat-a strike, poke; ga•-tingdat-a kick something, as a football, push with the feet; nang-ting-data bump into, as into a tree when walking; cha-ting-dat-a bump into, collide, bump something with one's body. (9.552)

ting-gil-ting-gil adv. loudly, of talking, crying, laughing. (3.628)

ting-krang-a vt. open, as an umbrella; hold out flat, of cloth. (9.60)

ting-ku $n$. rafter, (A·pal). (5.632)

ting-ku-ting-ku adv. loud, of a rice pounder, a squealing pig, of angry talking. (3.628)

ting-pret, ting-pret-ting-pret $a d v$. loudly, of crying or shouting, of bursting or burning bamboo. ting-pret-a vi. (7.29)

ting-ting $a d v$. built strongly against thieves, of walls, fences. (9.536)

ting-ting-a, wa-sing ting-ting-a vt. pound into, as into a bamboo tube. (5.458)

ting-ting-rok-rok-a, ting-ting-pak-rok-a vi. complete, thorough: ting-ting-pak-rok-e chok-a write down everything; ting-ting-rokrok-e cha-jok ate up everything. (3.343)

ting-wil-wil-a vt. surround, as with the walls of a house. (9.536)

ting-a vi. deep, of water, of holes; far in from an edge: mik-tik-kol ting-a have deep or sunken eyes, as when tired. (2.213)

ting-nap-a vi. enter deeply, as into a forest. (9.313)

ting-ting-a, gi-sik ting-ting-a vi. anxious, worried. (7.764)

-tip-, -tip-tip- aa. cover: ka-tip-a cover by tying something over; rim-tip-a cover with the hands, esp. to cover one's mouth in embarrassment; cover the nose against a smell; cover a hole, a pot, glass, etc.; 
mit-tip-a cover over, plug up; dok-tip-tip-a pat, slap several times; wen-tip-tip-a wind around with a lot of turns, thoroughly. (9.62)

tip-a vi. smoke heavily, of a fire; ai-au wal--ku tip-ing-a, bang-en my gosh, the fire is smoking a lot. (5.422)

tip-a vi. dry up, stop raining, stop flowing, of puddles, pools, streams, rain, breast, well, etc. (9.273)

-tip-tip- $a a$. thoroughly, repeatedly: dok-tip-tip-a pat, hit several times, as to soothe a child; ka-tip-tip-a tie thoroughly; ba-ji-tip-tip-a thoroughly tangled; wen-tip-tip-a very tangled; chot-tip-tip-a tear (e.g. string) to pieces. (3.342)

tip-tip-a vi. tangled, with much winding, of string, etc. (3.718)

to-a vi. good tasting: ja-ba to-be-a the curry tastes very good; to-e nik-a like the taste of. -to- $a a$. good for the senses: ni-to-a beautiful to look at; kin-a-to-a beautiful to hear; cha-to-a taste good to eat; ring-to-a taste good to drink; jak-kal-to-a good to use, comfortable to use. (3.682)

to-a, to-a gan-a, tu-a gan-a vi. do evil magic, with medicines rather than with a ritual chant, in order to cause someone to die. (8.566)

to-a, tu-a vt. measure the length, weight, etc. (9.66)

to-bu, to-bu-o-ba, to-bu-ba, tao, tao-ba, to-be, to-ba conj. but anyway, in spite of. $<$ B (1.4)

to-kai-a vt. cheat, overcharge, not pay back money that is owed. $<\mathrm{B}$ $(8.33)$

to-ma-to, to-me-to, to-mo-to $n$. tomato, (same as ba-ring bel-a-ti). $<\mathrm{E}(5.23)$

to-nik-a, to-e nik-a $v t$. like the taste of. (3.682)

to-pi, tu-pi $n$. hat, cap. $<$ B $(6.522)$

to-pi gil-gep $n$. hat with a visor. (6.522)

to-ra, tu-ra $n$. basket. (5.852)

to-ra a-ma, tu-ra a-ma $n$. large rice basket. (5.852)

to-re-a, tu-ri-a, tu-ri-grik-a vi. have sexual relations. (6.72)

to-re-kok, tu-ri-kok $n$. rapist. (8.33)

to-rom $n$. religious denomination. $<\mathrm{B}(8.51)$

to-sok, tu-sok $n$. mattress. $<\mathrm{B}(6.591)$

to-song-e ni-a, tu-song-e ni-a vt. measure depth of water with a pole. $(9.66)$

to--jot $a d v$. startled. (7.763)

-tok- aa. all, everything: nik-tok-a see everything; nam-tok-a all are good. (3.343)

tok-a vi. spotted, as a leopard or a pattern on cloth. vt. apply a spot, as colored cosmetic forehead spot, blood on an altar, flour spots on walls at festivals. (3.538) 
tok-a vt. apply a spot, as colored cosmetic spot to the forehead, blood on an altar, flour spots on walls at festivals. vi. spotted, as a leopard, a pattern on cloth. (6.565)

tok-a vt. catch by a sudden motion, as one catches an insect under the hand, as a cat leaps to surprise its prey. (9.531)

tok-ki-a vi. lose, as at sports or a legal case. $<\mathrm{B}(8.81)$

tok-kol $n$. a separate upright bamboo piece of an altar, a vertical bamboo post. (8.58)

tok-ta $n$. wooden board, plank. <B (5.636)

tol-a $n$. a large and dark kind of bamboo that is good for making baskets. $<\mathrm{B}(4.637)$

tol-op $n$. a course of mud laid down in building a house. tol-op cls. classifier for courses of mud laid down in house building. (5.638)

tol-si, tol-si-gip-bok $n$. 1 meter tall spicy smelling plant with purple or dark green leaves, and many small green or dark purple flowers in whorls on $12 \mathrm{~cm}$. stalks. (5.28)

tol-a vi. say something untrue, whether by error or to deceive, $(\mathrm{A} \cdot \mathrm{chik})$. $(7.25)$

tol-a vt. roll up. (9.645)

tol-chep-a vt. bunch up, roll cloth in a bunch. (9.645)

tol-rep-rep-a $v t$. roll something small between the palms. (9.645)

tol-rim-rim-a vt. bunch up, roll up in the hands. (9.645)

-tom- $a a$. round, make round: jak-tom-a make a fist; ka-tom-a tie in a bundle. tom- cls. for bellies, round things. -tom dns. round, bunched: jak-tom fist; dik-tom large round pot; a-tom stomach. (2.319)

tom-a vt. lift up, lift out, as a baby from a cloth cradle, hold in the hands. $(9.532)$

tom-ba ba-ring $n$. a large round type of ba-ring (eggplant). (5.23)

tom-be-tom-be, tom-bi-tom-bi $a d v$. crowded together, as in a bundle, as fruit on a branch. (2.25)

tom-dal-a vi. big, of round bunches, balls of string, cloth bundles: do-ri wen-jok-o do-ri tom-dal-ing-a when the string is wound, the string gets big and round. (2.211)

tom-sa, a-tom tom-sa, bi-bil tom-sa $n$. of one womb, having the same mother, true siblings. (6.634)

tom-a vt. gather, bunch up, collect, call together, pile up, rake into a pile, as rice; wind up thread; pull up, gather up, gather into a bunch, of a sleeve, pant leg, lunggi, etc.; bend together, of legs, waist or, esp., arms. $(9.535)$

tom-bil-a vt. make something swell up, as when a potter makes a pot, when ants make nests. (9.649)

tom•-bil-bil-a vt. bunch up, crumple up, as cloth, paper. (6.598) 
tom•-do-a vt. gather upward, bunch upward, of cloth, lunggi, shirt. $(9.535)$

tom•-e chu-a $v p h$. sleep with arms folded. (9.343)

tom•-pri-tom•-pri $a d v$. piece by piece, when breaking or tearing into pieces. (9.284)

tom-rep-rep-a $v t$. bunch up. (9.645)

tom-rop-rop-a $v t$. form into a ball, as paper, leaves, mud. (9.645)

tom-tom-a, gi-sik tom•-tom-a vi. calm, peaceful, without worries. (7.764)

ton-tro $n$. echo in mon-tro ton-tro ritual chant. $<\mathrm{B}(8.566)$

tong-kru, tong-ku, ting-ku $n$. rafter. (5.632)

tong-reng-ma gip-peng-pang $a d v$. speaking from opposite sides when disputing at a bi-char (legal meeting). (7.39)

tong- cls. classifier for lengths, pieces cut crosswise, of bamboo, wood, firewood, part of a broken pencil, lengthwise section, slices of a banana. tong- $a a$. cut or separate, esp. of long and cylindrical pieces: den-tonga cut crosswise, cut in two, of a log, etc.; tet-tong-a break apart, of string, linear things; chik-tong-a bite off. -tong dns. long and cylindrical: gan-tong a cut stalk of a plant such as manioc; ging-tong snout of a pig; jak-tong forearm; wa-tong length of bamboo. (1.32)

tong-dal-a vi. big around, of long cylindrical things, sticks, tails. (2.211) tong•-kan-di $a d v$. partial, unfinished; incomplete, as a bamboo without a top, half of a bamboo pole, butt of a cigarette, a life cut short by death before proper time. (3.347)

tong-kap-a vi. abbreviated, shortened of speaking; short of objects such as a shoulder bag, of distance along a road. tong·-kap, tong·-kaptong-kap adv. quickly, briefly, as speaking in an abbreviated way. $(2.215)$

tong-pri-tong•-pri adv. into bits, into small pieces. (3.347)

tong-rang-tong-rang $a d v$. into several crosswise slices. (9.573)

tong-tang $a d v$. straight, direct, of a road; short cut. -tong-tang- $a a$. direct, straight, not round about, short cut, abbreviated: bat-tong-tang-a take a short cut; kat-tong-tang-a run straight, directly. tong-tangtong-tang, tong-tang-ga-tang $a d v$. briefly, abbreviated. (2.318)

-tong-tang- $a a$. direct, straight, not roundabout, short cut, abbreviated; bat-tong-tang-a take a short cut; kat-tong-tang-a run straight, directly; tong-tang-tong-tang, tong-tang-ga-tang $a d v$. briefly, abbreviated. (0.42)

tong•-tang-e, tong-tang dak-e vt. crosswise, clear across: tong•-tange den-a, tong•-tang dak-e den-a cut across. tong•-tang-tong•-tang $a d v$. clear across. (3.347)

tong-tang-ga-tang $a d v$. in an abbreviated, shortened, way. (7.14) 
tong-tang-tong-tang $a d v$. made short or brief, as when speaking, as of pieces cut crosswise. (7.14)

top-a vi. collect, of water; swell up, of a burn, blister. (9.279)

top-a $v t$. build a hanging hive, by bees. (4.482)

top-top-a vt. pat with the hand, as to soothe a child. (9.552)

tor-mus $n$. watermelon. $<\mathrm{B}(5.23)$

tot- cls. classifier for drops, freckles, anthills, heads, mountains. (1.38)

tot-a vi. knock against, hit against, strike against, bump heads; mes tot-a strike a match. (9.553)

tot-a, chi tot-a vi. drip. (9.276)

tot-chon-a vi. small, of heads, of lumps of earth. (2.211)

tot-chrak-a vt. strike forcefully, as a match. (5.424)

tot-dal-a vi. large, of ant hills, tubers, mountains, and esp. heads, big headed. (2.211)

tot-grik-a vt. bump heads against each other, as one might do to two other people. (9.553)

tot-ma-tot-ma $a d v$. big, of tubers, mountains, etc., used for more than one. $(2.211)$

tot-ting-a vi. be lumpy, bumpy. (2.319)

tot-tot-a vi. drip, leak slightly. (9.276)

trak $n$. truck. $<\mathrm{E}(5.86)$

trak bal-ang-ga $n$. open truck. (5.86)

tring-trang $a d v$. thoroughly torn, into pieces, of cloth, etc. -tringtrang- $a a$. thoroughly, completely: ru-tring-trang-a take completely apart, of roof, fence, etc.; git-chit-tring-trang-a torn in many places, tattered. (9.292)

-trip-trip- $a a$. by many, collectively: cha-trip-trip-a eat by many, as insects eat wood; chik-trip-trip-a bite by many, as bees sting people; dok-trip-trip-a hit by many. (3.342)

trit-a $v t$. weave tightly, of a basket or cloth, so that adjacent pieces touch; pull up tight, of a sleeve, cloth, a tied string. (5.856)

tro, tru $n$. era, generation, belonging to the same era, of the same age. tro-ska, tru-ska, tro-sang-ga $n$. contemporaries, people of same age, same generation. (2.51)

tu-a-sim-ak-a vi. wake up, (A·pal). (7.97)

tu-chak-ram $n$. sleeping space, $(\mathrm{A} \cdot$ chik). (7.96)

tu-ra, to-ra $n$. basket, (Mandi). (5.852)

tu-re-tu-chik-gop-a vi. in the position for sex. (9.343)

tu-si-a vi. sleep, $(\mathrm{A} \cdot \mathrm{chik}) .(7.96)$

tu-sok, to-sok $n$. mattress. $<\mathrm{B}(6.591)$

tu-stim-a vi. nod off to sleep, doze, (A·pal). (7.96)

tu-tu-rak-rak $a d v$. startled, jump with fright. (7.763) 
tu-ta-ban-ta $a d v$. stutter, stammer. (7.15)

tul $n$. a stool that is higher than the ordinary kind of am-pok. $<\mathrm{B}$ $(5.82)$

tum-rang-tum-bit-chang $n$. a kind of striped snake, (same as chongbrang-chong-mit-chang. (4.443)

tup $n$. tube, of a tire. $<\mathrm{E}(5.872)$

tup-il-a $n$. bundle of things tied in a cloth. $<\mathrm{B}(6.521)$

u-a dem. that, it; combining form u-: u-cha den-bo cut with that;

$\mathbf{u}^{\bullet}$-ni sam-ba-o beside that. $\mathbf{u}-\mathbf{a}$ int. pause form, used when hesitating. $(1.5)$

u-a, o-a vi. open, of doors, etc. (see o- for compounds). (9.211)

u-a-dang, u-a-drang, u-a-rang, u-dang, u-drang dem. those. (1.5)

u-a-mang pro. they, (A·chik). (1.62)

u-bit-chet-a vi. dried out before ripening, of fruits and vegetables that remain on dying plants. (4.696)

u-dang, u-drang, u-a-dang, u-a-drang, u-a-rang dem. those. (1.5)

u-du-a-da $n$. a kind of owl that calls $\mathbf{u}-\mathbf{d u}$ at night, (brown fish-owl). (4.43)

u-du-ri-a vt. wear a cloth, such as one's sari, over one's head in modesty. (6.53)

u-gal, u-gar $n$. platform for rice or other things, storage area. $<\mathrm{B}(5.638)$

u-hu-bu-hu $a d v$. having lots of work, too much work. (8.433)

$\mathbf{u}-\mathbf{o}, \mathbf{u} \cdot \mathbf{- o}, \mathbf{u n} \cdot \mathbf{- o} a d v$. there, at that place. Locative of $\mathbf{u}-\mathbf{a}$. (2.13)

u-pol $n$. a kind of fish, (same as sai-tan). (4.454)

u-ru, hu-ru, ha-mak u-ru $n$. gibbon. (4.418)

u-tak-ka-ru $n$. large, three-holed bamboo flute. $<\mathrm{B}(8.85)$

u-cha bak $p p p$. on that side, there. (2.13)

u'-ni so-moi-o $a d v$. at that time, then. (2.51)

u'-ni-ko, u-ni-ko conj. besides, in addition, and then. (1.4)

u'-ni-ku-no, u-ni-ku-no conj. in addition. (1.4)

$\mathbf{u} \cdot \mathbf{- n o}, \mathbf{h u} \cdot \mathbf{- n o}, \mathbf{u}-\mathbf{n o}$ conj. then, and then, at that time. (1.4)

$\mathbf{u} \cdot \mathbf{- n o}, \mathbf{u n}-\mathbf{o}, \mathbf{u}-\mathbf{a}-\mathbf{n o}, \mathbf{u}-\mathbf{a}-\mathbf{o}, \mathbf{u}-\mathbf{o}$ dem. there, at that place. (2.13)

ui-a vt. know, (A·chik). (7.41)

ul-guk-a vi. noise made by tiger. (4.476)

ul-tai-a $v t$. turn over, upset. $<\mathrm{B}(9.214)$

$\mathbf{u n}-\mathbf{o}, \mathbf{u} \cdot \mathbf{- n o}, \mathbf{u}-\mathbf{o} a d v$. there, at that place. Locative of $\mathbf{u}-\mathbf{a}$. (2.13)

ut-tor $n$. north. $<\mathrm{B}(4.218)$

wa $n$. tooth, teeth on the edge of a cutting tool: ka-chi-wa teeth on the edge of a ka-chi (sickle) blade. (6.22)

wa bi-am-bong $n$. the middle section of a length of bamboo, between the root and the top. (4.670)

wa wil-a vi. the feeling of one's teeth after eating sour things, (lit. sharpen the teeth). (3.682) 
wa-a vi. rain. (4.325)

wa-bek-bek-a, wa-pak-pak-a vi. rain for a long time. (4.327)

wa-chi, wa-chi-ka-ri $n$. rainy season. (2.84)

wa-ching $n$. tusk, of an elephant or pig, canines of a dog. (4.473)

wa-chrek-chrek adv. with teeth showing. (9.44)

wa-chu $n$. front teeth, incisors and canines. (6.22)

wa-da $n$. echo in wa-chi wa-da rainy season. (2.84)

wa-dang-ku-dang $a d v$. speaking quickly and loudly, as when arguing, or shouting angrily. (7.15)

wa-drak-a, mik-ka wa-drak-a vi. rain heavily. (4.327)

wa-gam $n$. molars, back teeth. (6.22)

wa-gim-a vi. the feeling of the teeth when eating sour things. (3.682)

wa-grang-a vi. nicked, of an axe, or knife blade but less continuously nicked than bi-rik-ken-a; widely spaced, with gaps, as teeth may have gaps. (9.282)

wa-kam-a vi. rain continuously. (4.327)

wa-keng-keng $a d v$. with teeth showing. (9.44)

wa-ki $n$. tooth dirt, tartar, (lit. tooth-dung). (6.29)

wa-min-a vi. dull, blunt, not sharp. (3.666)

wa-ra-chak-a vt. defend someone, resist, deny someone else's guilt. (7.39)

wa-ram-a vi. show teeth, as when talking. (9.44)

wa-rap-a $n$. upper rows of thatch. (5.632)

wa-rek-rek, wa-re-rek $a d v$. in a straight line. (2.318)

wa-ring $n$. gums of the mouth. (6.22)

wa-si-a $n$. the second row of thatch just above the eaves. (5.632)

wa-wil-a $v t$. echo in $\mathbf{k u} \cdot$-ching-a wa-wil-a threaten. (8.36)

wa'-, wa, wa'-a $n$. bamboo. wa'- $c p$. prefix for bamboo parts, varieties, and object made from bamboo: wa'-ma a large variety of bamboo; wa'-si split bamboo matting; wa-kop one half of a split length of bamboo. (4.637)

wa•-al, wal•-, wal $n$. fire, (see wal-- for compounds). (5.421)

wa'-chek-si $n$. twig of bamboo. (4.671)

wa-dang $n$. length of bamboo, often decorated and with leaves still attached, that is used as part of an altar in a sacrifice. (8.58)

wa-ding $n$. thin flexible strips of bamboo that are used for tying, coarser than the strips used to make baskets. (5.639)

wa-gan-tong $n$. length of bamboo. (5.639)

wa-go-ra $n$. thick growing bamboo. (4.637)

wa·-gong $n$. horizontal bamboo pole hung outside, for drying clothes; well sweep. (5.82)

wa-ja-ting $n$. roots of bamboo. (4.678)

wa-kan-ta $n$. a thorny variety of bamboo that grows wild, (Beng. kantai). $<\mathrm{B}(4.637)$ 
wa-kap-kap $n$. a device made from two bamboos that clap together to scare away birds. (5.835)

wa-ki-chong-prot $n$. a bird, (hoopoe). (4.43)

wa-kop $n$. half split length of bamboo, lengthwise bamboo pieces that are tied above and below the tong-kru (rafters) on to which thatch is tied. (5.639)

wa-ku-rang $n$. half split length of bamboo. (5.639)

wa'-mi-sol $n$. closely spaced layer of bamboo floor support in a bu-rang (field house), above the dam-beng and just below the wa'-si. (5.639)

wa'-ni be-ra $n$. bamboo wall. (5.638)

wa'-ni kun-cha $n$. small side branches of bamboo. (4.671)

wa'-pang $n$. bamboo 'trunk', stalk of bamboo. (4.637)

wa-pek $n$. bamboo part that joins an a-du-ri (musical horn) to its handle. (8.85)

wa-rik-i $n$. the top part of growing bamboo. (4.637)

wa-rong $n$. bamboo lengths spaced close together, as the top layer of a floor support in a field house. (5.639)

wa-ru-ri $n$. a kind of bamboo said to grow in the Garo Hills. (4.637)

wa-si $n$. bamboo which has been cut at the nodes and flattened, and which is used to make walls and floors of old style houses, split bamboo. (5.638)

wa-si-nok $n$. house built of split bamboo. (5.622)

wa'-si-ri, wa'-sri $n$. split length of bamboo used for various purposes including further splitting to make flexible tie strips; bamboo part of a pong-si (divining bow). (5.639)

wa-sim n. a kind of bamboo, said to grow in Agartola. (4.637)

wa'-sing, wa'-ni wa'-sing $n$. bamboo cup, bamboo tube used for storage or for blowing up fire. (5.438)

wa-srep $n$. small variety of bamboo said to grow in the Garo Hills. (4.637)

wa-ti-ri, wa'-tri $n$. a smallish variety of bamboo, about $4 \mathrm{~cm}$. diameter. (4.637)

wa'-tong $n$. length of bamboo. (5.639)

wak n. pig. (5.35)

wak be-en $n$. pork, (lit. pig-meat). (5.401)

wak dok-a $n$. a celebration in which grown children thank their parents for the gift of land. (8.566)

-wak- aa. scoop: bak-wak-a scoop out dirt with a hoe; chok-wak-a scoop water (with hand, plate, spoon, etc.); su-wak-a scrape up dirt. $(9.576)$

-wak- $a a$. soft, spoiled: ka-wak-a too strong in taste, too much pepper, salt, etc.: chi-wak-a cooked too soft, of tubers, etc.; re-wak-a soiled, dirty from ink, dust, etc. (3.684) 
wak-bring $n$. a kind of wild pig, different from the domesticated wakman-di. (4.419)

wak-gal-a vi. vomit, (A•chik). (8.97)

wak-kin-te, wak-kin-ti $n$. kind of tree that looses leaves in the cold season, (Beng. but-tum). (4.632)

wak-man-di $n$. a small variety of domestic pig that is no longer common. $(5.35)$

wak-ring-kong $n$. feeding trough for pigs. (5.835)

wak-si-ri $n$. strips of pork fat. (5.401)

wak-wak-a $a d v$. eat very quickly. (5.472)

wal $n$. night. wal-a vi. become night. wal- cls. classifier for nights. $(2.52)$

wal, wal--, wa'-al $n$. fire, (see wal-- for compounds). (5.421)

wal seng-a vi. grow light at dawn, (lit. night brightens). (2.52)

wal so-gip-a, wal su'-gip-a $n p h$. bamboo device to make fire by friction. (5.423)

wal-chu-a, wal-o chu-a vi. spend the night, as when one sleeps at a kinsman's house. (7.96)

Wal-jam $n$. Serejing's boyfriend, from well-known songs. (8.83)

wal-jat-chi, wal-jang-chi $n$. midnight. (2.52)

wal-kim-bak-a $a d v$. half the night, late at night. (2.53)

wal-nam int. a somewhat artificial translation of English 'good night'. $(0.64)$

wal-ni $n$. morning, this morning. (2.52)

wal-ni gro-gro, wal-ni gru-gru $n$. the time at or following sunrise. $(2.52)$

wal-ni-ni $n$. early morning. (2.52)

wal-ni-wal-ni, wal-ni-rik-kit $a d v$. every morning. (2.71)

wal-pak-sa $a d v$. half the night, part of the night. (2.53)

wal-pil-a vi. come back, return. vt. return, as a loan, give back. (9.315) wal-seng-a vi. lie awake at night, unable to sleep. (7.97)

wal-si-sal-si $a d v$. all night and all day. (2.53)

wal-si-si $a d v$. all night. (2.53)

wal-tat $a d v$. whole night. (2.53)

wal--, wal, wa'-al $n$. fire. wal-- $c p$. prefix for things connected with fire: wal-ku $n$. smoke; wal--tot $n$. device to start fire by friction; wal--kambi $n$. tips of flames; wal-ki $n$. burning coals; wal-ki-ra-ra $n$. lots of burning coals, glow strongly; wal•-mi-si $n$. spark; wal--si-ri $n$. flames, (lit. fire-tongue). (5.421)

wal-bi-ma $n$. match. (5.423)

wal-chak-a, wal chak-a vi. warm oneself or one's hands at the fire. (3.673) 
wal-du-ka-a $v t$. set the time for a ceremony, fix the date. (8.562)

wal-gu-si $n$. dark color left by smoke, as on the ceilings of houses; the color of smoked bamboo strips used in basket making. (5.422)

wal--jem $n$. firebrand, a bundle of lighted sticks, used for illumination or to carry fire to another place. (5.423)

wal--ku-a n. smoke. vi. to smoke, of a fire. (5.422)

wal--mik-a, mik-mik-a vt. smoke, cause smoke to swirl around, fan or blow smoke; wave smoke about as part of sa-sat-su'-a ritual; smoke out bees or animals; use smoke to blacken objects; smoke bananas to ripen them; smoke cows to be rid of mosquitoes: wal-ko mik-mik-a, mi-si ong-kat-kan make a mouse come out with smoke. (5.422)

wal'-mik-o $l w$. at a great distance, (lit. in smoke). (2.11)

wal--tot $n$. bamboo device for kindling fire by friction, match. (5.423)

Wan-a, Wan-gal-a vi. hold the traditional autumn festival that was the major annual ceremony of the Garos. Wan-gal-a n. (8.562)

wan-chi-gin-di $n$. yeast used for making bread. (5.405)

Wan-kim-bak-a, Wan-kel-tap-a vi. perform a partial or shortened Wan-gal-a festival. (8.562)

Wan-ma-a, Wan-mang-song-a vi. do the full form of the Wan-gal-a festival. (8.562)

wan-ti, chu-wan-ti, wan-chi, chu-wan-chi, man-ti, chu-man-ti $n$. yeast cake, yeast used for making rice beer; used also to make spots on sacrificial altars. (5.405)

wang-a, ku-wang-a vi. open mouth wide, open mouthed, as in astonishment. -wang- $a a$. open wide: pa-wang-a open, of an umbrella; jak ba-wang-a hold the arms wide. -wang- $d n s$. open: ku-wang crazy person who lets mouth hang open. wang-wang $a d v$. open, spread apart, as shirts, doors. (9.44)

wang-chak-a vi. open mouth to receive food from another. (9.44)

wang-gip-a, a-wang $n$. father's younger brother, mother's younger sister's husband, stepfather. (6.611)

wang-sa $n$. uncle and niece or nephew. (6.62)

wat-a $v t$. weave basket, work bamboo. (5.856)

wat-a, wat-et-a $v t$. let go, release; settle, finalize; fire from a job; quit smoking; send a letter, etc.; let animals run loose; abandon, throw away. (9.72)

wat-chit-tik-a $v t$. weave a closely woven basket. (5.856)

wat-gring-grang-a $v t$. weave a basket with open spaces. (5.856)

we-a, wi-a $v t$. plow: a-ba-ko hal-cha we-a plow the fields with a plow. (5.154)

we-gil-gil-a, wi-gil-gil-a $v t$. plow shallowly. (2.213) 
-wek- $a a$. soft: go-wek-a throw mud; a-wek-a soft. dil`-wek'-dil-wek $a d v$. good and ripe, soft, of fruit. da-wek-da-wek $a d v$. cooked mushily. (3.664)

wek-a-wek-a, wek-wak-a vi. cooked very soft, as for babies or for old people without teeth. -wek- $a a$. soft: go-wek-a throw mud; a-wek-a soft. dil'-wek-dil-wek $a d v$. good and ripe, soft, of fruit: da-wek-dawek cooked mushily. wek-wak-ga-wak $a d v$. cooked very soft. (5.403)

wek-wak-a vi. noisy, with much talking. (3.624)

wel-a vi. pass noon, or pass midnight. (2.52)

wel--wel-a vi. talk lots, talk continuously, as when drunk. (7.14)

wel--wel-a vi. wind around, of a road that goes around a hill, of a winding group of rice fields. (2.315)

wen-a wen-wen-a $v t$. wind, as string, wrap around, wind around with a lot of turns, thoroughly wind around and around: do-ri-ko wen-wena wind the string. -wen-, -wen-wen- $a a$. wind around, go around in circles: kat-wen-wen-a run around; bil-wen-a fly in circles. (9.65)

wen-chet-a vt. wind around, of a snake, of a vine that winds around a tree. (2.315)

wen-tip-tip-a, wen-wen-tip-tip-a $a d v$. wrap something long, as a bandage; tangled, of string, wind thoroughly around and around. wen-wentip-tip $a d v$. (3.718)

weng ku-weng adv. arguing, disputing. (8.32)

weng.- cls. classifier for lengths of bamboo between two nodes. (1.37)

wi-a, we-a $v t$. plow. (5.154)

wi-sal $n$. whistle used in football. $<\mathrm{E}(8.85)$

wi-a vt. clean excrement. (6.57)

-wil-, -wil-wil- aa. in circles: kat-wil-wil-a run in circles; an`-sengwil-wil-a dance in circles; rat-wil-wil-a cut around, as cutting stripes around a handle; ting-wil-wil-a surround, as the walls of a house; dongwil-wil-a surround, by people. (9.327)

wil-a, wil-grop-a vi. fall over, as crops in the wind. (9.212)

wil-it-a vt. knock over, of growing rice. (9.513)

wil-wil-a vi. turn around, revolve, go in circles; circle around others, surround, all around. -wil-, -wil-wil- $a a$. in circles kat-wil-wil-a run in circles; an-seng-wil-wil-a dance in circles; rat-wil-wil-a cut around, as cutting stripes around a handle; ting-wil-wil-a surround, as by the walls of a house; dong-wil-wil-a surround, by people. (9.327)

wil-wil-dap-a $v t$. wrap around, as with a cloth or rope. (9.65)

wil-wil-e $a d v$. on all sides, surrounding, around the outside, as rice in beer pot; in circles. (2.11)

wil-a $v t$. sharpen, sharpen tools against a stone. (3.666) 